\title{
Regio- and Stereoselective Synthesis of Dispiro-bisoxindoles via [3+2] Annulation Involving Nitroisatylidene as a Vinylogous Michael Donor
}

\author{
Chenikkayala Siva Sankara and Irishi N. N. Namboothiri* \\ Department of chemistry, Indian Institute of Technology Bombay, Mumbai 400076, India
}

Table of contents

\begin{tabular}{|l|l|l|}
\hline Entry & Content & Page \\
\hline 1 & General information & S1 \\
\hline 2 & General procedure for the preparation of 3-(1-nitroalkylidene)-2-oxindole & S1-4 \\
\hline 3 & $\begin{array}{l}\text { General procedure for the synthesis of dispiro-bis-oxindole- } \gamma \text {-nitro esters and } \\
\text { spiro-fused oxindoles }\end{array}$ & S4-12 \\
\hline 4 & General procedure for gram scale synthesis of dispiro-bis-oxindole- $\gamma$-nitro esters & S12-13 \\
\hline 5 & General procedure for the synthesis of $\gamma$-amino esters & S13 \\
\hline 6 & $\begin{array}{l}\text { Selected X-ray crystal data for } \mathbf{1 g}, \mathbf{3 m}, \mathbf{3 s} \text { ', } \mathbf{4} \text { with 50\% probability for the ellipsoid } \\
\text { contour }\end{array}$ & $\mathrm{S} 14-17$ \\
\hline 7 & Selected torsional angles & S18-19 \\
\hline 8 & References & S19-20 \\
\hline
\end{tabular}

1. General Information. The melting points recorded are uncorrected. NMR spectra ${ }^{1} \mathrm{H},{ }^{1} \mathrm{H}$-decoupled ${ }^{13} \mathrm{C},{ }^{1} \mathrm{H}-{ }^{1} \mathrm{H}$ COSY, ${ }^{1} \mathrm{H}-{ }^{1} \mathrm{H}$ NOESY and ${ }^{1} \mathrm{H}_{-}{ }^{13} \mathrm{C} \mathrm{HSQC}$ were recorded with TMS as the internal standard. The coupling constants ( $\mathrm{J}$ values) are given in hertz. High resolution mass spectra were recorded under ESI Q-TOF conditions. X-ray data were collected on a diffractometer equipped with graphite monochromated Mo K $\alpha$ radiation. The structure was determined by direct methods shelxs 97 and refined by full-matrix least-squares against F2 using shelx197 software. All the 3-Alkylideneoxindole carboxylates 2 are known in literature and prepared using reported procedures. ${ }^{1}$ The 3 -olefinic-(1cyano)-2-oxindole,,${ }^{1 \mathrm{f}}$ trifluoromethyl-3-alkylideneoxindole, ${ }^{2}$ maleimides, ${ }^{3}$ were prepared by literature methods. Nitroalkene $\mathbf{1 h}$ and intermediate $\mathbf{1 1}$ are known in the literature. ${ }^{4 \mathrm{c}}$ Other analogs $\mathbf{1 a - g}$ are new compounds and were prepared by the general procedure reported in the literature. ${ }^{4 a-b}$

\section{Experimental section}

\section{General procedure for the preparation of 3-(1-nitroalkylidene)-2-oxindole $1^{\mathrm{a}, \mathrm{b}, \mathrm{c}}$}

To a solution of isatin $\mathbf{1 0}$ ( $2 \mathrm{mmol}, 1.0$ equiv) in $20 \mathrm{~mL}$ (excess) of nitroalkane was added piperidine (0.4 mmol, $20 \mathrm{~mol} \%)$ at $\mathrm{rt}$, and continued stirring for $15-30 \mathrm{~min}$ at the same temperature. After 
completion of the reaction (monitored by TLC), the reaction mixture was concentrated in vacuo until dryness. To the resulting crude nitroaldol in $25 \mathrm{~mL}$ of dry DCM were added $\mathrm{Et}_{3} \mathrm{~N}$ (6 mmol, 3.0 equiv) slowly and $\mathrm{SOCl}_{2}\left(6 \mathrm{mmol}, 3.0\right.$ equiv) dropwise over a period of $10 \mathrm{~min}$ at $0{ }^{\circ} \mathrm{C}$. The resulting reaction mixture was stirred for another $45 \mathrm{~min}$ at the same temperature. After completion of the reaction $50 \mathrm{~mL}$ water and $25 \mathrm{~mL}$ of saturated aqueous $\mathrm{NH}_{4} \mathrm{Cl}$ solution were added to the reaction mixture and stirring continued for another $10 \mathrm{~min}$. The aqueous layer was extracted with DCM $(3 \times 20 \mathrm{~mL})$ and the combined organic layer was dried over $\mathrm{Na}_{2} \mathrm{SO}_{4}$ and concentrated in vacuo. The crude residue was purified by silica gel column chromatography by gradient elution with petroleum ether/ethyl acetate (97/3 to 94/6), which offered the 3-(1-nitroalkylidene)-2-oxindoles (1a-g).
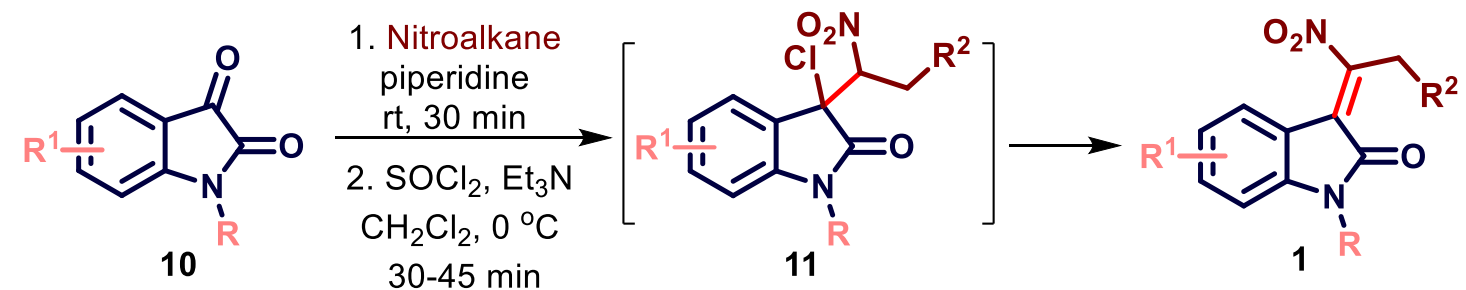

$\mathrm{R}=\mathrm{H}, \mathrm{CH}_{3}, \mathrm{Bn}, \mathrm{CH}_{2} \mathrm{CO}_{2} \mathrm{Et} ; \mathrm{R}^{1}=\mathrm{H}, \mathrm{CH}_{3}, \mathrm{OCH}_{3}, \mathrm{~F} ; \mathrm{R}^{2}=\mathrm{H}, \mathrm{CH}_{3}$
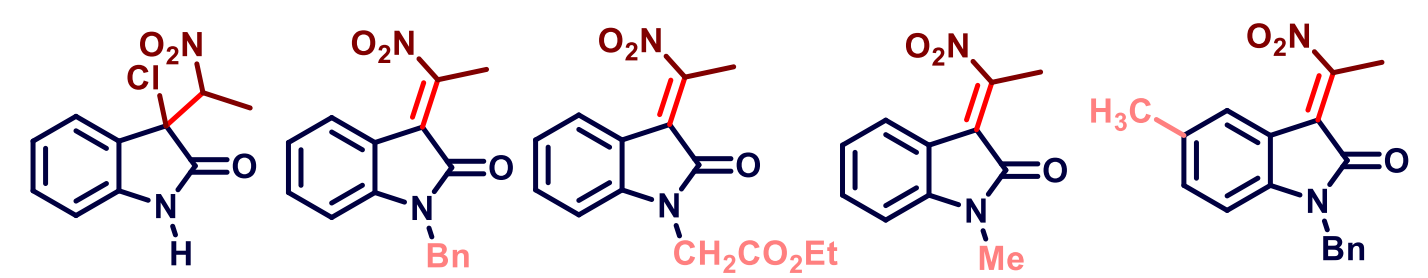

$11,72 \%$

1a, $61 \%$

1b, $32 \%$

1c, $42 \%$

1d, $46 \%$

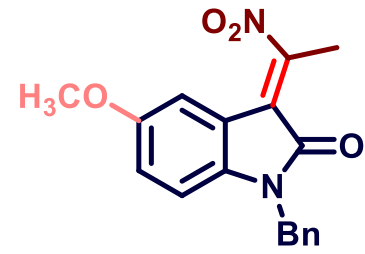

1e, $42 \%, 10: 1 d r$

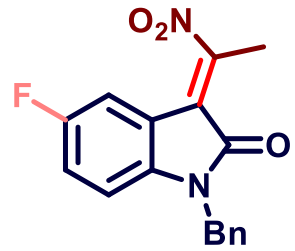

1f, $38 \%$

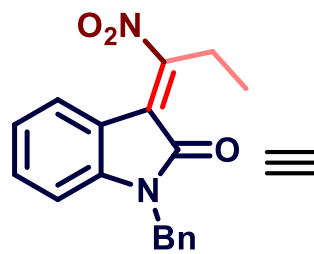

$1 \mathrm{~g}, 32 \%$

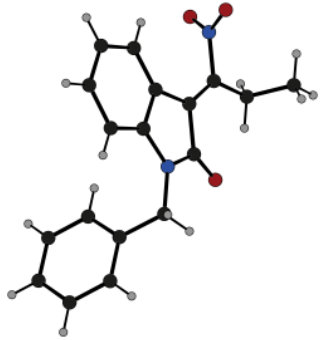

CCDC 2034250

${ }^{a}$ Reactions were performed on $2.0 \mathrm{mmol}$ of isatin 10 in $20 \mathrm{~mL}$ (excess) of nitroalkane with $20 \mathrm{~mol} \%$ of piperidine at room temperature for $15-30 \mathrm{~min}$. ${ }^{\text {b}}$ Yield after silica gel column chromatography. ${ }^{\mathrm{c}}$ Diatsereomeric ratios were determined by ${ }^{1} \mathrm{H} \mathrm{NMR}$ analysis of crude reaction mixture.

3-Chloro-3-(1-nitroethyl)indolin-2-one (11). Compound isolated using silica gel column chromatography by eluting with 75/25 petroleum ether/ethyl acetate; White solid;<smiles>CC(N)C1(Cl)C(=O)Nc2ccccc21</smiles>
Yield 346 mg, 72\%; mp 118-120 ${ }^{\circ} \mathrm{C}$; IR (KBr film) 3250 (br s), 1731 (vs), 1619 (s), 1557 (s), 1537 (m), 1473 (m), 1358 (m), 1336 (w), 1193 (w), 752 (s) cm ${ }^{-1}$; ${ }^{1} \mathrm{H}$ NMR $\left(500 \mathrm{MHz}, \mathrm{CDCl}_{3}\right) \delta 1.79(\mathrm{~d}, J=6.7 \mathrm{~Hz}, 3 \mathrm{H}), 5.29(\mathrm{q}, J=6.7 \mathrm{~Hz}, 1 \mathrm{H}), 6.97(\mathrm{~d}, J=$ $7.4 \mathrm{~Hz}, 1 \mathrm{H}), 7.13(\mathrm{t}, J=7.4 \mathrm{~Hz}, 1 \mathrm{H}), 7.35(\mathrm{t}, J=7.4 \mathrm{~Hz}, 1 \mathrm{H}), 7.53(\mathrm{~d}, J=7.4 \mathrm{~Hz}$, $1 \mathrm{H}), 9.00(\mathrm{~s}, 1 \mathrm{H}) ;{ }^{13} \mathrm{C}\{\mathrm{H}\} \mathrm{NMR}\left(125 \mathrm{MHz}, \mathrm{CDCl}_{3}\right)$ 15.3, 62.4, 87.5, 111.3, 124.1, 125.7, 125.8, 131.7, 140.6, 173.1; HRMS (ESI) m/z: $[\mathrm{M}+\mathrm{Na}]^{+}$Calcd for $\mathrm{C}_{10} \mathrm{H}_{9} \mathrm{ClN}_{2} \mathrm{O}_{3} \mathrm{Na}$ 263.0194; Found 263.0194. Although this compound is reported in ref $7 \mathrm{c}$, experimental data is not available. 
(E)-1-Benzyl-3-(1-nitroethylidene) indolin-2-one (1a). Compound isolated using silica gel column chromatography by eluting with $95 / 5$ petroleum ether/ethyl acetate; Red solid;<smiles>CC(=C1C(=O)N(Cc2ccccc2)c2ccccc21)[N+](=O)[O-]</smiles>
Yield $360 \mathrm{mg}, 61 \%$; mp 112-114 ${ }^{\circ} \mathrm{C}$; IR (KBr film) 3057 (m), 3031 (m), $2971(\mathrm{~m})$, 1712 (vs), 1656 (m), 1610 (vs), 1525 (s), 1360 (vs), 1352 (vs), 1190 (m), 1110 (m), 991 (s), $744(\mathrm{~s}) \mathrm{cm}^{-1} ;{ }^{1} \mathrm{H}$ NMR $\left(400 \mathrm{MHz}, \mathrm{CDCl}_{3}\right) \delta 2.90(\mathrm{~s}, 3 \mathrm{H}), 4.94(\mathrm{~s}, 2 \mathrm{H}), 6.73$ $(\mathrm{d}, J=7.8 \mathrm{~Hz}, 1 \mathrm{H}), 6.97(\mathrm{t}, J=7.8 \mathrm{~Hz}, 1 \mathrm{H}), 7.20-7.26(\mathrm{~m}, 2 \mathrm{H}), 7.27-7.35(\mathrm{~m}, 5 \mathrm{H})$; ${ }^{13} \mathrm{C}\{\mathrm{H}\}$ NMR $\left(100 \mathrm{MHz}, \mathrm{CDCl}_{3}\right) 16.4,43.9,109.6,117.5,119.1,123.1,123.3$, 127.4, 128.0, 129.0, 131.6, 135.4, 142.5, 153.5, 167.3; HRMS (ESI) m/z: [M + Na ${ }^{+}$ Calcd for $\mathrm{C}_{17} \mathrm{H}_{14} \mathrm{~N}_{2} \mathrm{O}_{3} \mathrm{Na} 317.0897$; Found 317.0898.

(E)-Ethyl 2-(3-(1-nitroethylidene)-2-oxoindolin-1-yl) acetate (1b). Compound isolated using silica<smiles>CCOCN1C(=O)/C(=C(/C)[N+](=O)[O-])c2ccccc21</smiles>
gel column chromatography by eluting with 96/4 petroleum ether/ethyl acetate; Pale red solid; Yield $188 \mathrm{mg}, 32 \%$; mp 136-138 ${ }^{\circ} \mathrm{C}$; IR (KBr film) 2987 (w), 2945 (w), 1735 (vs), 1723 (vs), 1655 (w), 1610 (m), 1535 (s), 1380 (m), 1355 (s), 1221 (s), 1197 (s), 1022 (m), $740(\mathrm{~m}) \mathrm{cm}^{-1} ;{ }^{1} \mathrm{H}$ NMR (400 MHz, $\left.\mathrm{CDCl}_{3}\right) \delta$ $1.25(\mathrm{t}, J=7.1 \mathrm{~Hz}, 3 \mathrm{H}), 2.83(\mathrm{~s}, 3 \mathrm{H}), 4.21(\mathrm{q}, J=7.1 \mathrm{~Hz}, 2 \mathrm{H}), 4.46(\mathrm{~s}, 2 \mathrm{H}), 6.69$ $(\mathrm{d}, J=7.7 \mathrm{~Hz}, 1 \mathrm{H}), 6.99(\mathrm{t}, J=7.7 \mathrm{~Hz}, 1 \mathrm{H}), 7.20(\mathrm{~d}, J=7.7 \mathrm{~Hz}, 1 \mathrm{H}), 7.29(\mathrm{t}, J$ $=7.7 \mathrm{~Hz}, 1 \mathrm{H}) ;{ }^{13} \mathrm{C}\{\mathrm{H}\} \mathrm{NMR}\left(100 \mathrm{MHz}, \mathrm{CDCl}_{3}\right) 14.2,16.3,41.4,62.1,108.6$, 117.4, 118.8, 123.3, 123.4, 131.6, 141.9, 153.5, 167.1, 167.3; HRMS (ESI) m/z: $[\mathrm{M}+\mathrm{Na}]^{+}$Calcd for $\mathrm{C}_{14} \mathrm{H}_{14} \mathrm{~N}_{2} \mathrm{O}_{5} \mathrm{Na} 313.0795$; Found 313.0795.

(E)-1-Methyl-3-(1-nitroethylidene)indolin-2-one (1c). Compound isolated using silica gel column<smiles>C/C(=C1/C(=O)N(C)c2ccccc21)[N+](=O)[O-]</smiles>
chromatography by eluting with $94 / 6$ petroleum ether/ethyl acetate; Red solid; Yield $185 \mathrm{mg}$, 42\%; mp 146-147 ${ }^{\circ} \mathrm{C}$; IR (KBr) 2940 (w), 1716 (vs), 1665 (w), 1608 (m), 1536 (s), $1491(\mathrm{w}), 1473(\mathrm{w}), 1419(\mathrm{w}), 1378(\mathrm{~m}), 1367$ (s) cm ${ }^{-1} ;{ }^{1} \mathrm{H} \mathrm{NMR}\left(400 \mathrm{MHz}, \mathrm{CDCl}_{3}\right)$ $\delta 2.83(\mathrm{~s}, 3 \mathrm{H}), 3.20(\mathrm{~s}, 3 \mathrm{H}), 6.78(\mathrm{~d}, J=7.8 \mathrm{~Hz}, 1 \mathrm{H}), 6.96(\mathrm{t}, J=7.8 \mathrm{~Hz}, 1 \mathrm{H}), 7.16(\mathrm{~d}$, $J=7.8 \mathrm{~Hz}, 1 \mathrm{H}), 7.31(\mathrm{t}, J=7.8 \mathrm{~Hz}, 1 \mathrm{H}) ;{ }^{13} \mathrm{C}\{\mathrm{H}\} \mathrm{NMR}\left(125 \mathrm{MHz}, \mathrm{CDCl}_{3}\right) 16.2,26.2$, 108.6, 117.3, 119.2, 122.9, 123.2, 131.6, 143.2, 153.1, 167.0; HRMS (ESI) m/z: [M + $\mathrm{H}]^{+}$Calcd for $\mathrm{C}_{11} \mathrm{H}_{10} \mathrm{~N}_{2} \mathrm{O}_{3}$ 219.0761; Found 219.0761.

(E)-1-Benzyl-5-methyl-3-(1-nitroethylidene) indolin-2-one (1d). Compound isolated using silica gel column chromatography by eluting with $95 / 5$ petroleum ether/ethyl acetate; Pale red solid; Yield 286<smiles>C/C(=C1/C(=O)N(Cc2ccccc2)c2ccc(C)cc21)[N+](=O)[O-]</smiles>
mg, 46\%; mp 128-129 ${ }^{\circ} \mathrm{C}$; IR (KBr film) 3031 (vw), 2923 (m), 2852 (w), 1742 (s), 1712 (s), 1617 (m), 1534 (s), 1490 (s), 1346 (s), 1195 (m), 810 (m), 733 (m), $697(\mathrm{~m}) \mathrm{cm}^{-1} ;{ }^{1} \mathrm{H}$ NMR $\left(400 \mathrm{MHz}, \mathrm{CDCl}_{3}\right) \delta 2.25(\mathrm{~s}, 3 \mathrm{H}), 2.89(\mathrm{~s}, 3 \mathrm{H}), 4.92(\mathrm{~s}$, $2 \mathrm{H}), 6.61(\mathrm{~d}, J=8.0 \mathrm{~Hz}, 1 \mathrm{H}), 7.01(\mathrm{~s}, 1 \mathrm{H}), 7.03(\mathrm{~d}, J=8.0 \mathrm{~Hz}, 1 \mathrm{H}), 7.24-7.34$ $(\mathrm{m}, 5 \mathrm{H}) ;{ }^{13} \mathrm{C}\{\mathrm{H}\} \mathrm{NMR}\left(100 \mathrm{MHz}, \mathrm{CDCl}_{3}\right)$ 16.4, 21.3, 43.9, 109.4, 117.6, 119.4, 123.9, 127.4, 128.0, 129.1, 132.1, 132.7, 135.5, 140.3, 153.2, 167.4; HRMS (ESI) $\mathrm{m} / \mathrm{z}:[\mathrm{M}+\mathrm{Na}]^{+}$Calcd for $\mathrm{C}_{18} \mathrm{H}_{16} \mathrm{~N}_{2} \mathrm{O}_{3} \mathrm{Na} 331.1053$; Found 331.1057.

(E)-1-Benzyl-5-methoxy-3-(1-nitroethylidene) indolin-2-one (1e). Compound isolated using silica<smiles>COc1ccc2c(c1)/C(=C(\C)[N+](=O)[O-])C(=O)N2Cc1ccccc1</smiles>
gel column chromatography by eluting with $97 / 3$ petroleum ether/ethyl acetate; Pale red solid; Yield $270 \mathrm{mg}, 42 \%$; 10:1 dr (inseparable); mp 112-114 ${ }^{\circ} \mathrm{C}$; IR (KBr film) 2932 (w), 2837 (vw), 1739 (s), 1711 (vs), 1595 (m), 1534 (s), 1493 (s), 1374 (m), 1346 (s), 1295 (m), 1190 (s) cm ${ }^{-1} ;{ }^{1} \mathrm{H}$ NMR (400 MHz, $\left.\mathrm{CDCl}_{3}\right) \delta$ $2.90(\mathrm{~s}, 3 \mathrm{H}), 3.71(\mathrm{~s}, 3 \mathrm{H}), 4.90(\mathrm{~s}, 2 \mathrm{H}), 6.61(\mathrm{~d}, J=8.4 \mathrm{~Hz}, 1 \mathrm{H}), 6.77(\mathrm{~d}, J=8.4$ $\mathrm{Hz}, 1 \mathrm{H}), 6.80(\mathrm{~s}, 1 \mathrm{H}), 7.27-7.32(\mathrm{~m}, 5 \mathrm{H}) ;{ }^{13} \mathrm{C}\{\mathrm{H}\}$ NMR (100 MHz, $\left.\mathrm{CDCl}_{3}\right)$ 

167.1; HRMS (ESI) m/z: [M + H] $]^{+}$Calcd for $\mathrm{C}_{18} \mathrm{H}_{17} \mathrm{~N}_{2} \mathrm{O}_{4}$ 325.1183; Found 325.1182.

(E)-1-Benzyl-5-fluoro-3-(1-nitroethylidene) indolin-2-one (1f). Compound isolated using silica gel column chromatography by eluting with $94 / 6$ petroleum ether/ethyl acetate; Pale red solid; Yield 240

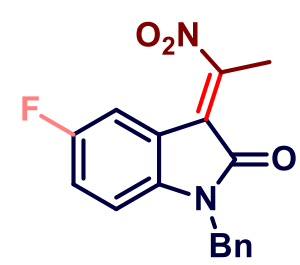
mg, 38\%; mp 112-114 ${ }^{\circ} \mathrm{C}$; IR (KBr film) 3064 (vw), 3033 (vw), 2923 (w), 1716 (vs), $1662(\mathrm{w}), 1618(\mathrm{w}), 1602(\mathrm{w}), 1536$ (s), $1484(\mathrm{~s}), 1456(\mathrm{~m}), 1374(\mathrm{~m}), 1343$ (s), $1180(\mathrm{~s}), \mathrm{cm}^{-1} ;{ }^{1} \mathrm{H}$ NMR $\left(500 \mathrm{MHz}, \mathrm{CDCl}_{3}\right) \delta 2.91(\mathrm{~s}, 3 \mathrm{H}), 4.93(\mathrm{~s}, 2 \mathrm{H}), 6.64$ $(\mathrm{dd}, J=8.4,4.2 \mathrm{~Hz}, 1 \mathrm{H}), 6.93-6.98(\mathrm{~m}, 2 \mathrm{H}), 7.26-7.35(\mathrm{~m}, 5 \mathrm{H}) ;{ }^{13} \mathrm{C}\{\mathrm{H}\} \mathrm{NMR}$ $\left(125 \mathrm{MHz}, \mathrm{CDCl}_{3}\right) 15.7,43.3,109.5(\mathrm{~d}, J=8.8 \mathrm{~Hz}), 110.6(\mathrm{~d}, J=26.3 \mathrm{~Hz}), 117.4$ $(\mathrm{d}, J=23.8 \mathrm{~Hz}), 117.7(\mathrm{~d}, J=10.0 \mathrm{~Hz}), 118.2(\mathrm{~d}, J=2.5 \mathrm{~Hz}), 126.6,127.4,128.4$, 134.3, 137.9, 153.8, $158.4(\mathrm{~d}, J=238.8 \mathrm{~Hz}), 166.3 ;{ }^{19} \mathrm{~F} \mathrm{NMR}\left(470 \mathrm{MHz}, \mathrm{CDCl}_{3}\right) \delta$-119.4; HRMS (ESI) $\mathrm{m} / \mathrm{z}:[\mathrm{M}+\mathrm{Na}]^{+}$Calcd for $\mathrm{C}_{17} \mathrm{H}_{13} \mathrm{FN}_{2} \mathrm{O}_{3} \mathrm{Na} 335.0802$; Found 335.0801.

(E)-1-Benzyl-3-(1-nitropropylidene)indolin-2-one (1g). Compound isolated using silica gel column chromatography by eluting with 96/4 petroleum ether/ethyl acetate; Yellow solid; Yield $200 \mathrm{mg}$, 32\%;

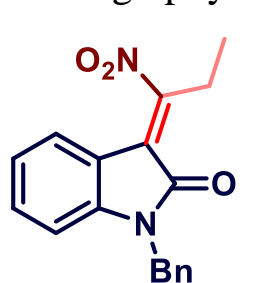
mp 108-110 ${ }^{\circ} \mathrm{C}$; IR (KBr film) 3062 (vw), 2974 (w), 2937 (w), 1712 (vs), 1655 (w), 1609 (s), 1532 (s), 1483 (m), 1470 (m), 1454 (m), 1360 (s), 1186 (m), 746 (m), 698 (m) $\mathrm{cm}^{-1} ;{ }^{1} \mathrm{H}$ NMR $\left(500 \mathrm{MHz}, \mathrm{CDCl}_{3}\right) \delta 1.28(\mathrm{t}, J=7.5 \mathrm{~Hz}, 3 \mathrm{H}), 3.44(\mathrm{q}, J=7.5$ $\mathrm{Hz}, 2 \mathrm{H}), 4.94(\mathrm{~s}, 2 \mathrm{H}), 6.73(\mathrm{~d}, J=7.8 \mathrm{~Hz}, 1 \mathrm{H}), 6.96(\mathrm{t}, J=7.8 \mathrm{~Hz}, 1 \mathrm{H}), 7.19(\mathrm{~d}, J=$ $7.8 \mathrm{~Hz}, 1 \mathrm{H}), 7.23(\mathrm{t}, J=7.8 \mathrm{~Hz}, 1 \mathrm{H}), 7.26-7.35(\mathrm{~m}, 5 \mathrm{H}) ;{ }^{13} \mathrm{C}\{\mathrm{H}\} \mathrm{NMR}(125 \mathrm{MHz}$, $\left.\mathrm{CDCl}_{3}\right) 11.5,22.9,43.9,109.6,117.6,118.3,123.1(\times 2), 127.4,128.0,129.0,131.5$, 135.4, 142.5, 159.0, 167.1; HRMS (ESI) m/z: $[\mathrm{M}+\mathrm{Na}]^{+}$Calcd for $\mathrm{C}_{18} \mathrm{H}_{16} \mathrm{~N}_{2} \mathrm{O}_{3} \mathrm{Na}$ 331.1053; Found 331.1057; Selected X-ray Data (CCDC 2034250): $\mathrm{C}_{18} \mathrm{H}_{16} \mathrm{~N}_{2} \mathrm{O}_{3}, M$ 308.34, Monoclinic, space group

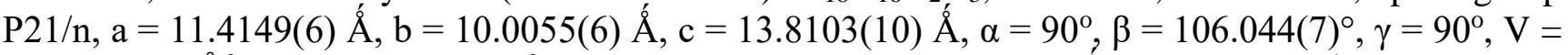
$1515.86(17) \AA^{3}, \mathrm{Dc}=1.3510 \mathrm{~g} / \mathrm{cm}^{3}, \mathrm{Z}=4, \mathrm{~F}(000)=648.3, \lambda=0.71073 \AA, \mu=0.093 \mathrm{~mm}^{-1}$, Total/ unique reflections $=20498 / 2666[\mathrm{R}($ int $)=0.1028], \mathrm{T}=\mathrm{N} / \mathrm{A}, \theta$ range $=4.12$ to $50^{\circ}$, Final $\mathrm{R}[\mathrm{I}>2 \sigma(\mathrm{I})]: \mathrm{R}_{1}=$ $0.0478, \mathrm{wR}_{2}=0.1115, \mathrm{R}$ (all data): $\mathrm{R}_{1}=0.0667, \mathrm{wR}_{2}=0.1266$.

\section{General procedure for the synthesis of di-spirocyclopentyl-3,3'-bisoxindoles/spiro-fused oxindoles $(3 / 4 / 7)$}
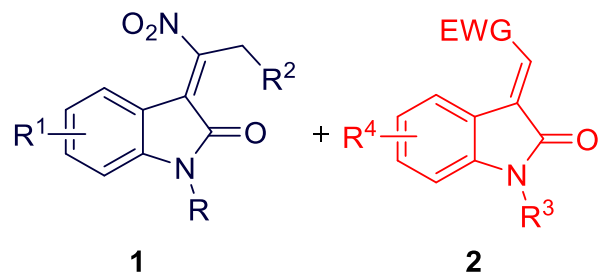

2
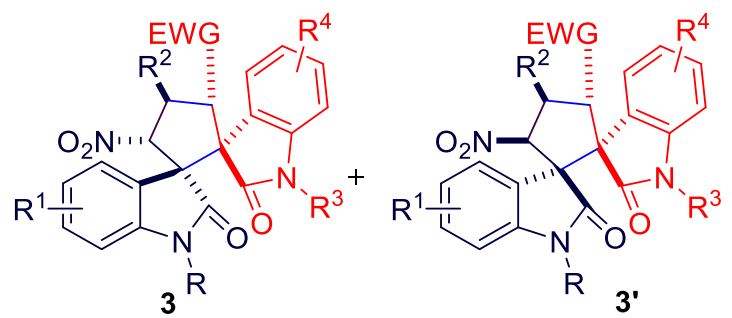

To a mixture of 3-(1-nitroalkylidene)-2-oxindole $\mathbf{1}$ (0.26 mmol, 1.3 equiv) and 3alkylideneoxindole/maleimide/2-indene-1,3-dione $2(0.2 \mathrm{mmol}, 1$ equiv $)$ in acetonitrile $(1.0 \mathrm{~mL})$ was added DABCO (2.5 mg, $0.02 \mathrm{mmol}, 0.1$ equiv) at room temperature. The resulting reaction mixture was stirred at the same temperature until completion (monitored by TLC) of the reaction. After completion, the reaction mixture was concentrated in vасио and the aqueous layer was extracted with ethyl acetate $(3 \times 10 \mathrm{~mL})$. The resulting combined organic layer was dried over $\mathrm{Na}_{2} \mathrm{SO}_{4}$ and concentrated in vacuo. The diastereomeric ratios were determined by ${ }^{1} \mathrm{H}$ NMR analysis of crude reaction mixtures. Thereafter, the crude residue was purified by silica gel column chromatography by gradient elution with petroleum ether/ethyl acetate (90/10 to $75 / 25)$ or fractional crystallization from ethanol and water (90:10), which provided the desired pure $\mathbf{3 / 4 / 7}$ in excellent yields. 
In most cases, the major diastereomer was isolated in pure form, but not the minor isomer, except in the case of $\mathbf{3 s}$. In the case of $\mathbf{3 s}$, both major and minor diastereomers were separated and fully characterized.

Ethyl 1,1"'-dibenzyl-5'-nitro-2,2' '-dioxodispiro[indoline-3,1'-cyclopentane-2',3''-indoline]-3'-

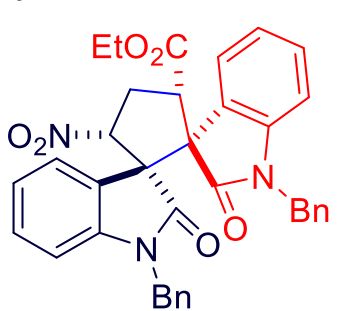
carboxylate (3a). White solid; Yield $118 \mathrm{mg}$, 98\%; 9:1 dr; Major isomer (isolated by fractional crystallization): $\mathrm{mp} 161-163{ }^{\circ} \mathrm{C}$; IR (KBr film) 2917 (vw), 1741 (s), 1713 (vs), 1611 (m), 1548 (s), 1490 (m), 1468 (w), 1371 (s), $1210(\mathrm{w}), 1181(\mathrm{w}), 756(\mathrm{vs}) \mathrm{cm}^{-1} ;{ }^{1} \mathrm{H}$ NMR $\left(400 \mathrm{MHz}, \mathrm{CDCl}_{3}\right) \delta 0.67$ (dd collapsed to t, $J=7.2 \mathrm{~Hz}, 3 \mathrm{H}$ ), 3.03 (ddd collapsed to dt, $J=12.3,7.8 \mathrm{~Hz}, 1 \mathrm{H}$ ), $3.68(\mathrm{dq}, J=10.8,7.2 \mathrm{~Hz}, 1 \mathrm{H}), 3.83(\mathrm{dq}, J=10.8,7.2 \mathrm{~Hz}, 1 \mathrm{H}), 3.88$ (overlapped dd, $J=12.3,7.8 \mathrm{~Hz}, 1 \mathrm{H}), 4.29(\mathrm{q}, J=12.3 \mathrm{~Hz}, 1 \mathrm{H}), 4.65,4.79$ $(\mathrm{ABq}, J=16.0 \mathrm{~Hz}, 2 \mathrm{H}), 4.69,4.86(\mathrm{~d}, J=16.1 \mathrm{~Hz}, 2 \mathrm{H}), 5.96(\mathrm{dd}, J=12.3,7.8 \mathrm{~Hz}, 1 \mathrm{H}), 6.41$ (overlapped $\mathrm{d}, J=7.8 \mathrm{~Hz}, 1 \mathrm{H}), 6.41$ (overlapped d, $J=7.6 \mathrm{~Hz}, 1 \mathrm{H}), 6.77-6.83(\mathrm{~m}, 5 \mathrm{H}), 6.87(\mathrm{t}, J=7.8 \mathrm{~Hz}, 1 \mathrm{H})$, 7.05-7.20 (m, 8H), $7.34(\mathrm{~d}, J=7.6 \mathrm{~Hz}, 1 \mathrm{H}), 7.46(\mathrm{~d}, J=7.8 \mathrm{~Hz}, 1 \mathrm{H}) ;{ }^{13} \mathrm{C}\{\mathrm{H}\} \mathrm{NMR}\left(100 \mathrm{MHz}, \mathrm{CDCl}_{3}\right)$ 13.6, 31.0, 43.9 (×2), 47.7, 60.5, $60.9(\times 2), 86.7,109.5,109.7,123.0,123.1,124.2,124.9,125.0,126.6$, 126.7, 127.0, 127.4, 127.6, 128.7, 128.8, 129.5, 129.7, 134.6, 135.0, 143.3, 144.0, 169.3, 173.8, 178.1; HRMS (ESI) m/z: [M + Na $]^{+}$Calcd for $\mathrm{C}_{36} \mathrm{H}_{31} \mathrm{~N}_{3} \mathrm{O}_{6} \mathrm{Na}$ 624.2105; Found 624.2104.

Ethyl 1"-benzyl-1-(2-ethoxy-2-oxoethyl)-5"'-methyl-5'-nitro-2,2"'-dioxodispiro[indoline-3,1'cyclopentane-2',3'-indoline]-3'-carboxylate (3b). White solid; Yield $115 \mathrm{mg}$, 94\%; 4.5:1 dr; Major

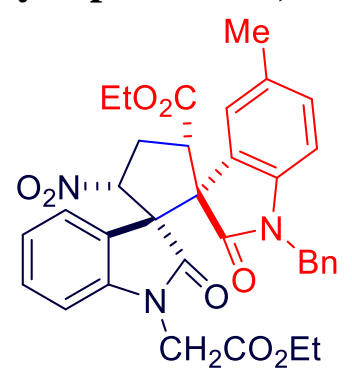
isomer (isolated by fractional crystallization): $\mathrm{mp} 209-211^{\circ} \mathrm{C}$; IR $(\mathrm{KBr}$ film) 2979 (m), 1741 (vs), 1718 (vs), 1613 (m), 1549 (s), 1496 (m), 1469 (w), 1371 (s), 1204 (s), 1029 (m), 756 (s) $\mathrm{cm}^{-1} ;{ }^{1} \mathrm{H}$ NMR (400 MHz, $\left.\mathrm{CDCl}_{3}\right) \delta 0.68$ (dd collapsed to t, $J=7.2 \mathrm{~Hz}, 3 \mathrm{H}$ ), 1.11 (t, $J=7.1 \mathrm{~Hz}, 3 \mathrm{H}$ ), 2.18 (s, 3H), 2.99 (ddd collapsed to dt, $J=12.3,7.9 \mathrm{~Hz}, 1 \mathrm{H}), 3.67(\mathrm{dq}, J=10.8,7.2 \mathrm{~Hz}, 1 \mathrm{H}), 3.76(\mathrm{dd}$, $12.3, J=7.9 \mathrm{~Hz}, 1 \mathrm{H}), 3.84(\mathrm{dq}, J=10.8,7.2 \mathrm{~Hz}, 1 \mathrm{H}), 3.99(\mathrm{dq}, J=10.7,7.1 \mathrm{~Hz}$, $1 \mathrm{H}), 4.04(\mathrm{dq}, J=10.7,7.1 \mathrm{~Hz}, 1 \mathrm{H}), 4.03(\mathrm{q}, J=12.3 \mathrm{~Hz}, 1 \mathrm{H}), 4.18,4.37(\mathrm{ABq}$, $J=17.2 \mathrm{~Hz}, 2 \mathrm{H}), 4.71,4.82(\mathrm{ABq}, J=16.1 \mathrm{~Hz}, 2 \mathrm{H}), 5.97(\mathrm{dd}, J=12.3,7.9 \mathrm{~Hz}$, $1 \mathrm{H}), 6.28(\mathrm{~d}, J=8.0 \mathrm{~Hz}, 1 \mathrm{H}), 6.53(\mathrm{~d}, J=7.8 \mathrm{~Hz}, 1 \mathrm{H}), 6.82-6.85(\mathrm{~m}, 3 \mathrm{H}), 6.89(\mathrm{t}, J=8.0 \mathrm{~Hz}, 1 \mathrm{H}), 7.05$ $(\mathrm{s}, 1 \mathrm{H}), 7.13-7.22(\mathrm{~m}, 4 \mathrm{H}), 7.44(\mathrm{~d}, J=8.0 \mathrm{~Hz}, 1 \mathrm{H}) ;{ }^{13} \mathrm{C}\{\mathrm{H}\} \mathrm{NMR}\left(125 \mathrm{MHz}, \mathrm{CDCl}_{3}\right)$ 13.6, 14.1, 21.1, 30.6, 41.5, 43.9, 47.6, 60.3, 60.4, 60.9, 61.7, 86.4, 108.6, 109.1, 123.3, 123.7, 124.3, 125.0, 126.7, 127.0, 127.6, 128.8, 129.6, 129.7, 132.6, 134.8, 140.7, 143.5, 167.0, 169.3, 173.5, 178.1; HRMS (ESI) m/z: $[\mathrm{M}+\mathrm{H}]^{+}$Calcd for $\mathrm{C}_{34} \mathrm{H}_{34} \mathrm{~N}_{3} \mathrm{O}_{8} 612.2340$; Found 612.2342.

Ethyl 1"-benzyl-5"'-methoxy-1-methyl-5'-nitro-2,2"'-dioxodispiro[indoline-3,1'-cyclopentane2',3"-indoline]-3'-carboxylate (3c). Pale Orange solid; Yield $102 \mathrm{mg}$, 92\%; 6:1 dr; Major isomer (isolated by fractional crystallization): $\mathrm{mp} 227-229^{\circ} \mathrm{C}$; IR (KBr film) $2961(\mathrm{~m})$,

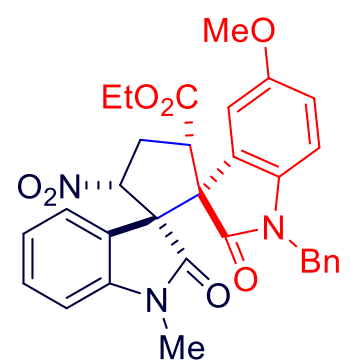
2938 (m), 2836 (vw), 1741 (s), 1708 (vs), 1609 (s), 1546 (s), 1495 (s), 1468 (s), 1453 (m), 1436 (m), 1372 (vs), 1355 (s), 1298 (m), 1270 (s), 1182 (m), 1097 (m), $763(\mathrm{~s}) \mathrm{cm}^{-1} ;{ }^{1} \mathrm{H}$ NMR $\left(500 \mathrm{MHz}, \mathrm{CDCl}_{3}\right) \delta 0.73(\mathrm{t}, J=7.0 \mathrm{~Hz}, 3 \mathrm{H}), 3.00$ (overlapped $\mathrm{m}$ and s, 4H), 3.68 (overlapped s, 3H), $3.72(\mathrm{dq}, J=10.0,7.0 \mathrm{~Hz}$, $1 \mathrm{H}), 3.77(\mathrm{t}, J=9.0 \mathrm{~Hz}, 1 \mathrm{H}), 3.86(\mathrm{dq}, J=10.0,7.0 \mathrm{~Hz}, 1 \mathrm{H}), 4.26(\mathrm{q}, J=12.0$ $\mathrm{Hz}, 1 \mathrm{H}), 4.69,4.80(\mathrm{ABq}, J=16.0 \mathrm{~Hz}, 2 \mathrm{H}), 6.01(\mathrm{dd}, J=11.5,8.0 \mathrm{~Hz}, 1 \mathrm{H}), 6.26$ $(\mathrm{d}, J=8.5 \mathrm{~Hz}, 1 \mathrm{H}), 6.56(\mathrm{~d}, J=7.5 \mathrm{~Hz}, 1 \mathrm{H}), 6.62(\mathrm{~d}, J=8.0 \mathrm{~Hz}, 1 \mathrm{H}), 6.82$ (overlapped $2 \times \mathrm{d}, J=6.5 \mathrm{~Hz}, 2 \mathrm{H}), 6.89(\mathrm{t}, J=7.5 \mathrm{~Hz}, 1 \mathrm{H}), 6.97(\mathrm{~s}, 1 \mathrm{H}), 7.16-$ 7.24 (unresolved m, 4H), $7.42(\mathrm{~d}, J=7.5 \mathrm{~Hz}, 1 \mathrm{H}) ;{ }^{13} \mathrm{C}\{\mathrm{H}\}$ NMR $\left(125 \mathrm{MHz}, \mathrm{CDCl}_{3}\right)$ 13.6, 26.1, 30.7, 43.9, 47.6, 56.0, 60.4, 60.6, 60.9, 86.3, 108.3, 109.8, 112.6, 115.0, 123.0, 123.9, 124.8, 125.7, 126.7, 127.6, 128.8, 129.7, 134.7, 136.5, 144.5, 155.9, 169.2, 173.6, 178.0; HRMS (ESI) m/z: $[\mathrm{M}+\mathrm{H}]^{+}$Calcd for $\mathrm{C}_{31} \mathrm{H}_{29} \mathrm{~N}_{3} \mathrm{O}_{7}$ 556.2074; Found 556.2078. 
Ethyl 1,1"'-dibenzyl-5,5"'-dimethyl-5'-nitro-2,2"-dioxodispiro[indoline-3,1'-cyclopentane-2',3"'indoline]-3'-carboxylate (3d). White solid; Yield $121.5 \mathrm{mg}, 96 \%$; 8:1 dr; Major isomer (isolated by

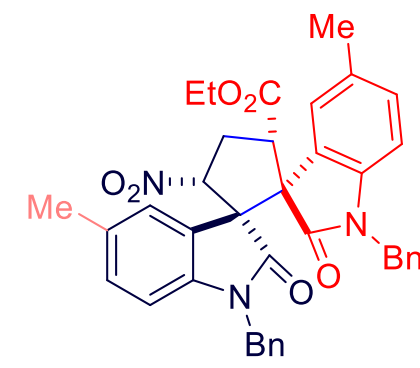
fractional crystallization): $\mathrm{mp} 182-184{ }^{\circ} \mathrm{C}$; IR (KBr film) 2977 (vw), 2928 (vw), 1715 (vs), 1611 (m), 1548 (s), 1490 (m), 1467 (m), 1369 (s), 1183 (w), $1154(\mathrm{~m}), 755(\mathrm{~s}) \mathrm{cm}^{-1} ;{ }^{1} \mathrm{H}$ NMR $\left(400 \mathrm{MHz}, \mathrm{CDCl}_{3}\right) \delta 0.69(\mathrm{t}, J=7.2$ $\mathrm{Hz}, 3 \mathrm{H}), 2.05$ (s, 3H), 2.09 (s, 3H), 3.01 (ddd collapsed to dt, $J=12.3,7.7$ $\mathrm{Hz}, 1 \mathrm{H}$ ), 3.69, 3.83 (ABqq, $J=10.8,7.2 \mathrm{~Hz}, 2 \mathrm{H}$ ), 3.85 (overlapped dd, $J=$ $12.3,7.7 \mathrm{~Hz}, 1 \mathrm{H}), 4.29(\mathrm{q}, J=12.3 \mathrm{~Hz}, 1 \mathrm{H}), 4.62(\mathrm{~d}, J=16.1 \mathrm{~Hz}, 1 \mathrm{H}), 4.65$ $(\mathrm{d}, J=16.2 \mathrm{~Hz}, 1 \mathrm{H}), 4.87(\mathrm{~d}, J=16.1 \mathrm{~Hz}, 1 \mathrm{H}), 4.96(\mathrm{~d}, J=16.2 \mathrm{~Hz}, 1 \mathrm{H})$, $5.97(\mathrm{dd}, J=12.3,7.7 \mathrm{~Hz}, 1 \mathrm{H}), 6.29$ (overlapped d, $J=7.9 \mathrm{~Hz}, 1 \mathrm{H}), 6.30$ (overlapped d, $J=7.9 \mathrm{~Hz}, 1 \mathrm{H}), 6.73(\mathrm{~d}, J=7.2 \mathrm{~Hz}, 2 \mathrm{H}), 6.80(\mathrm{~d}, J=7.2 \mathrm{~Hz}, 2 \mathrm{H}), 6.87$ (overlapped d, $J$ $=7.9 \mathrm{~Hz}, 1 \mathrm{H}), 6.89($ overlapped d, $J=7.9 \mathrm{~Hz}, 1 \mathrm{H}), 7.09-7.19(\mathrm{~m}, 7 \mathrm{H}), 7.28(\mathrm{~s}, 1 \mathrm{H})$; Confirmed by ${ }^{1} \mathrm{H}-$ ${ }^{1} \mathrm{H}$ COSY experiment; ${ }^{13} \mathrm{C}\{\mathrm{H}\}$ NMR $\left(100 \mathrm{MHz}, \mathrm{CDCl}_{3}\right)$ 13.6, 21.1, 21.2, 30.9, $43.8(\times 2), 47.6,60.5$ ( $\times 2), 60.9,86.7,109.3,109.4,124.0,124.8,125.6,126.2,126.5,127.2(\times 2), 127.3,128.6,128.7,129.6$, 129.9, 132.5, 132.7, 134.6, 135.1, 140.9, 141.6, 169.4, 173.8, 178.2; HRMS (ESI) m/z: $[\mathrm{M}+\mathrm{H}]^{+}$Calcd for $\mathrm{C}_{38} \mathrm{H}_{36} \mathrm{~N}_{3} \mathrm{O}_{6} 630.2599$; Found 630.2597 .

Ethyl 1,1"'-dibenzyl-5-methoxy-5"'-methyl-5'-nitro-2,2"'-dioxodispiro[indoline-3,1'-cyclopentane2',3''-indoline]-3'-carboxylate (3e). White solid; Yield $116 \mathrm{mg}, 90 \% ; 10: 1 \mathrm{dr}$; Major isomer (isolated by fractional crystallization): $\mathrm{mp}$ 202-204 ${ }^{\circ} \mathrm{C}$; IR (KBr film) 3030 (w), 2940 (w), 1741 (s), 1709 (vs),

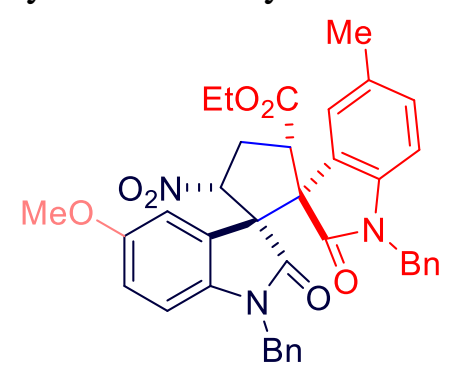
1603 (w), 1548 (s), 1496 (s), 1454 (m), 1436 (m), 1371 (s), 1352 (m), 1203 (s), $1179(\mathrm{~m}), 1029(\mathrm{w}), 811(\mathrm{w}), 759(\mathrm{~s}) \mathrm{cm}^{-1} ;{ }^{1} \mathrm{H} \mathrm{NMR}\left(500 \mathrm{MHz}, \mathrm{CDCl}_{3}\right)$ $\delta 0.68(\mathrm{t}, J=7.1 \mathrm{~Hz}, 3 \mathrm{H}), 2.06(\mathrm{~s}, 3 \mathrm{H}), 3.01$ (ddd collapsed to dt, $J=12.3$, $7.6 \mathrm{~Hz}, 1 \mathrm{H}), 3.54(\mathrm{~s}, 3 \mathrm{H}), 3.68(\mathrm{dq}, J=10.8,7.1 \mathrm{~Hz}, 1 \mathrm{H}), 3.72$ (overlapped $\mathrm{dq}, J=10.8,7.1 \mathrm{~Hz}, 1 \mathrm{H}$ ), 3.74 (overlapped dd, $J=12.3,7.6 \mathrm{~Hz}, 1 \mathrm{H}$ ), 4.30 $(\mathrm{q}, J=12.3 \mathrm{~Hz}, 1 \mathrm{H}), 4.60(\mathrm{~d}, J=16.1 \mathrm{~Hz}, 1 \mathrm{H}), 4.70(\mathrm{~d}, J=16.2 \mathrm{~Hz}, 1 \mathrm{H})$, 4.89 (overlapped d, $J=16.1 \mathrm{~Hz}, 1 \mathrm{H}), 4.92$ (overlapped d, $J=16.2 \mathrm{~Hz}, 1 \mathrm{H}$ ), $5.97(\mathrm{dd}, J=12.3,7.6 \mathrm{~Hz}, 1 \mathrm{H}), 6.30(\mathrm{~d}, J=8.5 \mathrm{~Hz}, 1 \mathrm{H}), 6.32(\mathrm{~d}, J=8.0$ $\mathrm{Hz}, 1 \mathrm{H}), 6.61(\mathrm{dd}, J=8.5,2.5 \mathrm{~Hz}, 1 \mathrm{H}), 6.79(\mathrm{~d}, J=7.2 \mathrm{~Hz}, 4 \mathrm{H}), 6.89(\mathrm{~d}, J=8.0 \mathrm{~Hz}, 1 \mathrm{H}), 7.10-7.20(\mathrm{~m}$, $8 \mathrm{H}) ;{ }^{13} \mathrm{C}\{\mathrm{H}\} \mathrm{NMR}\left(125 \mathrm{MHz}, \mathrm{CDCl}_{3}\right)$ 13.6, 21.1, 30.9, $43.8(\times 2), 47.7,55.7,60.5,60.7,60.9,86.8$, 109.3, 110.2, 111.9, 114.6, 124.9, 125.3, 126.4, 126.7, 127.3 (× 2), 127.5, 128.7, 128.8, 129.8, 132.9, 134.7, 135.2, 137.4, 141.0, 156.0, 169.3, 173.6, 178.2; HRMS (ESI) m/z: $[\mathrm{M}+\mathrm{H}]^{+}$Calcd for $\mathrm{C}_{38} \mathrm{H}_{36} \mathrm{~N}_{3} \mathrm{O}_{7}$ 646.2548; Found 646.2545.

Ethyl 1,1"'-dibenzyl-5-fluoro-5"'-methyl-5'-nitro-2,2"'-dioxodispiro[indoline-3,1'-cyclopentane2',3"-indoline]-3'-carboxylate (3f). White solid; Yield $118 \mathrm{mg}, 93 \% ; 8: 1 \mathrm{dr}$; Major isomer (isolated

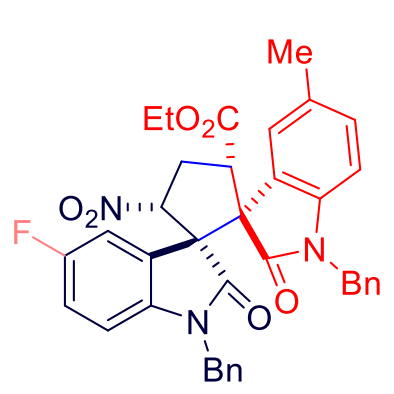
by fractional crystallization): $\mathrm{mp} 200.5-202.5^{\circ} \mathrm{C}$; IR (KBr film) 3031 (w), 1741 (s), 1713 (vs), 1617 (w), 1549 (s), 1495 (s), 1453 (m), 1371 (s), 1351 (m), $1271(\mathrm{w}), 1205(\mathrm{~m}), 1180(\mathrm{~m}), 1030(\mathrm{w}), 813(\mathrm{w}), 759(\mathrm{~s}) \mathrm{cm}^{-1} ;{ }^{1} \mathrm{H}$ NMR $\left(400 \mathrm{MHz}, \mathrm{CDCl}_{3}\right) \delta 0.67(\mathrm{t}, J=7.2 \mathrm{~Hz}, 3 \mathrm{H}), 2.06(\mathrm{~s}, 3 \mathrm{H}), 3.02$ (ddd collapsed to dt, $J=12.2,7.8 \mathrm{~Hz}, 1 \mathrm{H}), 3.68(\mathrm{dq}, J=10.8,7.2 \mathrm{~Hz}, 1 \mathrm{H}), 3.68$ $(\mathrm{dq}, J=10.8,7.2 \mathrm{~Hz}, 1 \mathrm{H}$ ), 3.85 (overlapped dd, $J=12.2,7.8 \mathrm{~Hz}, 1 \mathrm{H}$ ), 4.28 $(\mathrm{q}, J=12.2 \mathrm{~Hz}, 1 \mathrm{H}), 4.59(\mathrm{~d}, J=16.2 \mathrm{~Hz}, 1 \mathrm{H}), 4.78,4.84(\mathrm{q}, J=16.0 \mathrm{~Hz}$, 2H), $4.90(\mathrm{~d}, J=16.2 \mathrm{~Hz}, 1 \mathrm{H}), 5.95(\mathrm{dd}, J=12.2,7.8 \mathrm{~Hz}, 1 \mathrm{H}), 6.30(\mathrm{dd}, J$ $=8.6,4.3 \mathrm{~Hz}, 1 \mathrm{H}), 6.37(\mathrm{~d}, J=8.0 \mathrm{~Hz}, 1 \mathrm{H}), 6.76-6.81(\mathrm{~m}, 3 \mathrm{H}), 6.87-6.92$ $(\mathrm{m}, 3 \mathrm{H}), 7.10-7.20(\mathrm{~m}, 7 \mathrm{H}), 7.23(\mathrm{dd}, J=8.6,2.6 \mathrm{~Hz}, 1 \mathrm{H}) ;{ }^{13} \mathrm{C}\{\mathrm{H}\}$ NMR $\left(125 \mathrm{MHz}, \mathrm{CDCl}_{3}\right)$ 13.6, 21.1, $30.8,44.0$ (× 2), 47.7, 60.5, 60.6, 61.0, 86.8, 109.4, $110.2(\mathrm{~d}, J=7.5 \mathrm{~Hz}), 113.4(\mathrm{~d}, J=26.2 \mathrm{~Hz}), 116.1$ $(\mathrm{d}, J=22.5 \mathrm{~Hz}), 124.7,125.9(\mathrm{~d}, J=7.5 \mathrm{~Hz}), 126.6,126.7,127.3,127.5,127.7,128.8,128.9,130.0$, 133.0, 134.7, 134.8, $140.1(\mathrm{~d}, J=2.5 \mathrm{~Hz}), 141.0,159.2(\mathrm{~d}, J=240.0 \mathrm{~Hz}), 169.2,173.6,177.9 ;{ }^{19} \mathrm{~F} \mathrm{NMR}$ 
(470 MHz, $\mathrm{CDCl}_{3}$ ) $\delta$-119.1; HRMS (ESI) m/z: $[\mathrm{M}+\mathrm{H}]^{+}$Calcd for $\mathrm{C}_{37} \mathrm{H}_{33} \mathrm{FN}_{3} \mathrm{O}_{6}$ 634.2348; Found 634.2348 .

Ethyl 1,1"'-dibenzyl-4'-methyl-5'-nitro-2,2"'-dioxodispiro[indoline-3,1'-cyclopentane-2',3"-

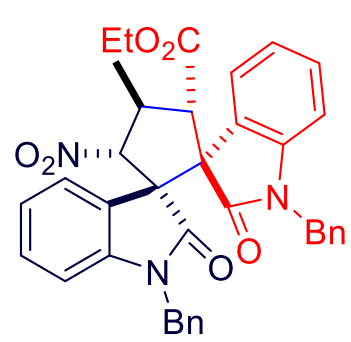
indoline]-3'-carboxylate (3g). White solid; Yield $41.5 \mathrm{mg}, 34 \% ; 8: 1 \mathrm{dr}$; Major isomer (compound isolated using silica gel column chromatography by eluting with 86/14 petroleum ether/ethyl acetate): $\mathrm{mp} 137-139{ }^{\circ} \mathrm{C}$; IR $(\mathrm{KBr}$ film) 3059 (w), 2932 (m), 1739 (vs), 1715 (vs), 1664 (w), 1610 (vs), 1535 (s), $1490(\mathrm{~m}), 1472$ (m), 1368 (s), 1343 (s), $1266(\mathrm{~m}), 1091(\mathrm{~m}), 747(\mathrm{~s}) \mathrm{cm}^{-1} ;{ }^{1} \mathrm{H}$ NMR (400 MHz, $\left.\mathrm{CDCl}_{3}\right) \delta 0.67(\mathrm{t}, J=7.2 \mathrm{~Hz}, 3 \mathrm{H}), 1.53(\mathrm{~d}, J=6.4 \mathrm{~Hz}, 3 \mathrm{H})$, $3.45(\mathrm{~d}, J=10.3 \mathrm{~Hz}, 1 \mathrm{H}), 3.67(\mathrm{dq}, J=10.7,7.2 \mathrm{~Hz}, 1 \mathrm{H}), 3.84(\mathrm{dq}, J=10.7$, $7.2 \mathrm{~Hz}, 1 \mathrm{H}), 4.64-4.73$ (overlapped $\mathrm{m}, 1 \mathrm{H}), 4.66,4.84(\mathrm{ABq}, J=16.0 \mathrm{~Hz}$, $2 \mathrm{H}), 4.73,4.81(\mathrm{ABq}, J=16.2 \mathrm{~Hz}, 2 \mathrm{H}), 5.65(\mathrm{~d}, J=11.7 \mathrm{~Hz}, 1 \mathrm{H}), 6.39$ (overlapped d, $J=7.7 \mathrm{~Hz}, 1 \mathrm{H})$, 6.41 (overlapped d, $J=7.6 \mathrm{~Hz}, 1 \mathrm{H}), 6.79-6.88(\mathrm{~m}, 6 \mathrm{H}), 7.05-7.21(\mathrm{~m}, 8 \mathrm{H}), 7.37(\mathrm{~d}, J=7.6 \mathrm{~Hz}, 1 \mathrm{H})$, $7.46(\mathrm{~d}, J=7.7 \mathrm{~Hz}, 1 \mathrm{H}) ;{ }^{13} \mathrm{C}\{\mathrm{H}\} \mathrm{NMR}\left(100 \mathrm{MHz}, \mathrm{CDCl}_{3}\right) 13.6,18.0,37.8,43.9(\times 2), 56.1,58.8,60.9$, 61.6, 92.2, 109.5, 109.6, 123.1 (× 2), 123.7, 125.0, 125.1, 126.7, 126.9, 127.0, 127.4, 127.6, 128.8, 128.9, 129.5, 129.8, 134.7, 135.1, 143.4, 143.9, 169.0, 173.4, 178.3; HRMS (ESI) m/z: $[\mathrm{M}+\mathrm{H}]^{+}$Calcd for $\mathrm{C}_{37} \mathrm{H}_{34} \mathrm{~N}_{3} \mathrm{O}_{6}$ 616.2442; Found 616.2440.

Methyl 1,1"'-dibenzyl-5'-nitro-2,2"'-dioxodispiro[indoline-3,1'-cyclopentane-2',3''-indoline]-3'carboxylate (3h). White solid; Yield $115 \mathrm{mg}$, 98\%; 5:1 dr; Major isomer

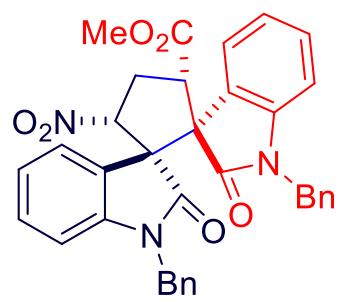
(isolated by fractional crystallization): $\mathrm{mp} 208-210{ }^{\circ} \mathrm{C}$; IR ( $\mathrm{KBr}$ film) 3028 (w), 2951 (vw), 1744 (s), 1712 (vs), 1610 (m), 1552 (m), 1488 (m), 1467 (w), 1370 (s), 1179 (w), 760 (s) $\mathrm{cm}^{-1} ;{ }^{1} \mathrm{H}$ NMR (400 MHz, $\left.\mathrm{CDCl}_{3}\right) \delta 3.05$ (ddd collapsed to dt, $J=12.3,7.9 \mathrm{~Hz}, 1 \mathrm{H}), 3.28(\mathrm{~s}, 3 \mathrm{H}), 3.85(\mathrm{dd}, J=12.3,7.9 \mathrm{~Hz}$, $1 \mathrm{H}), 4.29(\mathrm{q}, J=12.3 \mathrm{~Hz}, 1 \mathrm{H}), 4.64(\mathrm{~d}, J=16.0 \mathrm{~Hz}, 1 \mathrm{H}), 4.73,4.78(\mathrm{ABq}, J$ $=16.1 \mathrm{~Hz}, 2 \mathrm{H}), 4.93(\mathrm{~d}, J=16.0 \mathrm{~Hz}, 1 \mathrm{H}), 5.96(\mathrm{dd}, J=12.3,7.9 \mathrm{~Hz}, 1 \mathrm{H}), 6.42$ (overlapped d, $J=7.5 \mathrm{~Hz}, 1 \mathrm{H}$ ), 6.43 (overlapped d, $J=7.4 \mathrm{~Hz}, 1 \mathrm{H}), 6.76(\mathrm{~d}, J$ $=7.2 \mathrm{~Hz}, 2 \mathrm{H}), 6.81-6.87(\mathrm{~m}, 4 \mathrm{H}), 7.05-7.21(\mathrm{~m}, 8 \mathrm{H}), 7.31(\mathrm{~d}, J=7.4 \mathrm{~Hz}, 1 \mathrm{H}), 7.46(\mathrm{~d}, J=7.5 \mathrm{~Hz}, 1 \mathrm{H})$; ${ }^{13} \mathrm{C}\{\mathrm{H}\}$ NMR $\left(125 \mathrm{MHz}, \mathrm{CDCl}_{3}\right) 31.0,43.9,44.0,47.7,51.8,60.5(\times 2), 86.7,109.6,109.7,123.1(\times$ 2), 124.1, 124.7, 125.0, 126.6, 126.7, 127.0, 127.5, 127.6, 128.8, 128.9, 129.6, 129.7, 134.6, 135.0, 143.3, 144.0, 169.8, 173.8, 178.1; HRMS (ESI) m/z: $[\mathrm{M}+\mathrm{H}]^{+}$Calcd for $\mathrm{C}_{35} \mathrm{H}_{30} \mathrm{~N}_{3} \mathrm{O}_{6}$ 588.2129; Found 588.2126.

Tert-butyl 1,1"'-dibenzyl-5'-nitro-2,2"'-dioxodispiro[indoline-3,1'-cyclopentane-2',3"'-indoline]3'-carboxylate (3i). White solid; Yield $122.5 \mathrm{mg}$, 97\%; 4:1 dr; Major isomer (isolated by fractional

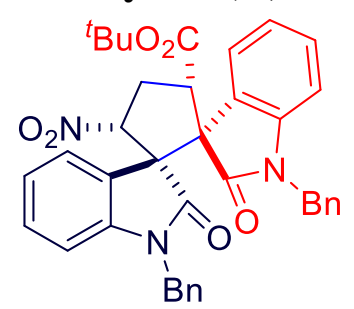
crystallization): $\mathrm{mp} 185-186{ }^{\circ} \mathrm{C}$; IR (KBr film) 3030 (vw), 2921 (vw), 1741 (s), 1710 (vs), 1617 (m), 1603 (m), 1548 (s), 1497 (s), 1453 (w), 1433 (m), 1370 (s), 1352 (s), 1202 (s), 1175 (m), 812 (m), 758 (s) cm ${ }^{-1} ;{ }^{1} \mathrm{H}$ NMR (400 $\left.\mathrm{MHz}, \mathrm{CDCl}_{3}\right) \delta 1.04(\mathrm{~s}, 9 \mathrm{H}), 3.00$ (ddd collapsed to dt, $J=12.2,7.4 \mathrm{~Hz}, 1 \mathrm{H}$ ), $3.83(\mathrm{dd}, J=12.2,7.4 \mathrm{~Hz}, 1 \mathrm{H}), 4.18(\mathrm{q}, J=12.2 \mathrm{~Hz}, 1 \mathrm{H}), 4.58(\mathrm{~d}, J=16.0$ $\mathrm{Hz}, 1 \mathrm{H}), 4.59(\mathrm{~d}, J=16.1 \mathrm{~Hz}, 1 \mathrm{H}), 4.82(\mathrm{~d}, J=16.0 \mathrm{~Hz}, 1 \mathrm{H}), 4.88(\mathrm{~d}, J=16.1$ $\mathrm{Hz}, 1 \mathrm{H}), 5.84(\mathrm{dd}, J=12.2,7.4 \mathrm{~Hz}, 1 \mathrm{H}), 6.40$ (overlapped d, $J=7.6 \mathrm{~Hz}, 1 \mathrm{H}$ ), 7.42 (overlapped d, $J=7.7 \mathrm{~Hz}, 1 \mathrm{H}), 6.71(\mathrm{~d}, J=7.5 \mathrm{~Hz}, 2 \mathrm{H}), 6.75(\mathrm{~d}, J=7.4 \mathrm{~Hz}, 2 \mathrm{H}), 6.83(\mathrm{t}, J=7.6$ $\mathrm{Hz}, 1 \mathrm{H}), 6.89(\mathrm{t}, J=7.6 \mathrm{~Hz}, 1 \mathrm{H}), 7.05-7.18(\mathrm{~m}, 8 \mathrm{H}), 7.43(\mathrm{~d}, J=7.7 \mathrm{~Hz}, 1 \mathrm{H}), 7.48(\mathrm{~d}, J=7.6 \mathrm{~Hz}, 1 \mathrm{H})$; ${ }^{13} \mathrm{C}\{\mathrm{H}\} \mathrm{NMR}\left(125 \mathrm{MHz}, \mathrm{CDCl}_{3}\right)$ 27.8, 31.4, $43.9(\times 2), 48.4,60.7,60.8,81.7,86.7,109.2,109.6,122.9$, 123.2, 124.6, $125.2(\times 2), 126.6,126.9,127.4,127.5,127.8,128.7,128.8,129.4,129.6,134.7,135.1$, 143.4, 144.0, 168.0, 173.9, 178.0; HRMS (ESI) m/z: $[\mathrm{M}+\mathrm{H}]^{+}$Calcd for $\mathrm{C}_{38} \mathrm{H}_{36} \mathrm{~N}_{3} \mathrm{O}_{6} 630.2599$; Found 630.2599 . 


\section{1,1'-Dibenzyl-5'-nitro-2,2"'-dioxodispiro[indoline-3,1'-cyclopentane-2',3''-indoline]-3'-}

carbonitrile (3j). White solid; Yield $109 \mathrm{mg}, 95 \%$; >19:1 dr; Major isomer (isolated by fractional crystallization): $\mathrm{mp} 209.5-211.5^{\circ} \mathrm{C}$; IR (KBr film) 3062 (w), $2920(\mathrm{w}), 2247$ (w), 1711 (v), 1612 (s),

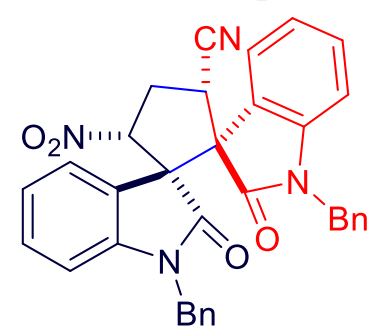
1548 (s), 1489 (m), 1468 (m), 1373 (s), $1181(\mathrm{~m}), 735(\mathrm{~m}), 698(\mathrm{~m}) \mathrm{cm}^{-1}$; ${ }^{1} \mathrm{H}$ NMR (400 MHz, $\mathrm{CDCl}_{3}$ ) $\delta 3.48$ (ddd collapsed to dt, $J=13.2,9.5 \mathrm{~Hz}$, $1 \mathrm{H}), 3.69(\mathrm{dd}, J=9.5,7.0 \mathrm{~Hz}, 1 \mathrm{H}), 3.87(\mathrm{ddd}, J=13.2,10.3,7.0 \mathrm{~Hz}, 1 \mathrm{H})$, $4.69(\mathrm{~d}, J=15.8 \mathrm{~Hz}, 1 \mathrm{H}), 4.72(\mathrm{~d}, J=15.7 \mathrm{~Hz}, 1 \mathrm{H}), 4.86(\mathrm{~d}, J=15.8 \mathrm{~Hz}$, $1 \mathrm{H}), 4.99(\mathrm{~d}, J=15.7 \mathrm{~Hz}, 1 \mathrm{H}), 6.23(\mathrm{dd}, J=10.3,9.5 \mathrm{~Hz}, 1 \mathrm{H}), 6.50$ (overlapped d, $J=7.7 \mathrm{~Hz}, 1 \mathrm{H}$ ), 6.53 (overlapped d, $J=7.8 \mathrm{~Hz}, 1 \mathrm{H}$ ), 6.78 (t, $J=7.7 \mathrm{~Hz}, 1 \mathrm{H}), 6.95(\mathrm{t}, J=7.8 \mathrm{~Hz}, 1 \mathrm{H}), 6.99-7.02(\mathrm{~m}, 2 \mathrm{H}), 7.09$ (t, $J=7.7$ $\mathrm{Hz}, 1 \mathrm{H}), 7.12-7.18(\mathrm{~m}, 3 \mathrm{H}), 7.21-7.29(\mathrm{~m}, 7 \mathrm{H}), 7.68(\mathrm{dd}, J=7.8 \mathrm{~Hz}, 1 \mathrm{H})$; Confirmed by ${ }^{1} \mathrm{H}-{ }^{1} \mathrm{H}$ COSY experiment; ${ }^{13} \mathrm{C}\{\mathrm{H}\}$ NMR $\left(125 \mathrm{MHz}, \mathrm{CDCl}_{3}\right)$ 32.3, 32.8, 44.3, 44.4, 58.9, 60.4, 85.5, 109.8, 110.1, 118.6, 121.6, 122.6, 123.3, 123.8, 124.0, 127.4, 127.5, 127.8, 128.1, 128.9, 129.0, $130.1(\times 2), 130.6$, 134.4, 134.8, 143.1, 144.0, 173.8, 176.9; HRMS (ESI) m/z: $[\mathrm{M}+\mathrm{H}]^{+}$Calcd for $\mathrm{C}_{34} \mathrm{H}_{26} \mathrm{~N}_{4} \mathrm{O}_{4} \mathrm{Na}$ 577.1846; Found 577.1846.

Ethyl 1,1'-dibenzyl-5"'-methyl-5'-nitro-2,2"'-dioxodispiro[indoline-3,1'-cyclopentane-2',3"'indoline]-3'-carboxylate (3k). White solid; Yield $122 \mathrm{mg}$, 99\%; 7:1 dr; Major isomer (isolated by fractional crystallization): $\mathrm{mp} 219-221{ }^{\circ} \mathrm{C}$; IR (KBr film) 3030 (w), 2920 (w), 1740 (s), 1712 (vs), 1612

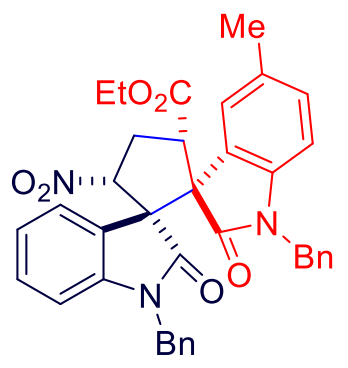
(m), 1548 (s), 1496 (m), 1370 (s), 1204 (m), 1175 (m), 755 (s) $\mathrm{cm}^{-1} ;{ }^{1} \mathrm{H}$ NMR $\left(400 \mathrm{MHz}, \mathrm{CDCl}_{3}\right) \delta 0.68$ (dd collapsed to t, $J=7.2 \mathrm{~Hz}, 3 \mathrm{H}$ ), 2.05 (s, 3H), 3.02 (ddd collapsed to dt, $J=12.3,7.5 \mathrm{~Hz}, 1 \mathrm{H}$ ), 3.69, 3.84 (ABqq, $J=10.7,7.2 \mathrm{~Hz}$, 2H), 3.86 (overlapped dd, $J=12.3,7.5 \mathrm{~Hz}, 1 \mathrm{H}), 4.29(\mathrm{q}, J=12.3 \mathrm{~Hz}, 1 \mathrm{H})$, $4.60,4.83(\mathrm{ABq}, J=16.0 \mathrm{~Hz}, 2 \mathrm{H}), 4.70,4.91(\mathrm{ABq}, J=16.4 \mathrm{~Hz}, 2 \mathrm{H}), 5.98$ $(\mathrm{dd}, J=12.3,7.5 \mathrm{~Hz}, 1 \mathrm{H}), 6.30(\mathrm{~d}, J=8.0 \mathrm{~Hz}, 1 \mathrm{H}), 6.40(\mathrm{~d}, J=7.8 \mathrm{~Hz}, 1 \mathrm{H})$, 6.76-6.81 (m, 4H), 6.87 (overlapped $2 \times \mathrm{t}, J=8.0 \mathrm{~Hz}, 2 \mathrm{H}), 7.06-7.20(\mathrm{~m}, 8 \mathrm{H})$, $7.46(\mathrm{~d}, J=8.0 \mathrm{~Hz}, 1 \mathrm{H})$; Confirmed by ${ }^{1} \mathrm{H}-{ }^{1} \mathrm{H}$ COSY, ${ }^{1} \mathrm{H}-{ }^{1} \mathrm{H}$ NOESY and ${ }^{1} \mathrm{H}-$ ${ }^{13} \mathrm{C}$ HSQC experiments; ${ }^{13} \mathrm{C}\{\mathrm{H}\}$ NMR $\left(125 \mathrm{MHz}, \mathrm{CDCl}_{3}\right)$ 13.6, 21.1, 31.0, 43.8, 43.9, 47.6, $60.5(\times 2)$, 60.9, 86.7, 109.3, 109.7, 123.0, 124.2, 124.9, 125.0, 126.6 (× 2), 127.3, 127.4, 127.5, 128.7, 128.8, 129.7, 129.8, 132.8, 134.7, 135.1, 140.9, 144.2, 169.4, 173.9, 178.1; HRMS (ESI) m/z: [M + H] $]^{+}$Calcd for $\mathrm{C}_{37} \mathrm{H}_{34} \mathrm{~N}_{3} \mathrm{O}_{6} 616.2442$; Found 616.2444.

Ethyl 1,1"'-dibenzyl-5"'-methoxy-5'-nitro-2,2"'-dioxodispiro[indoline-3,1'-cyclopentane-2',3"indoline]-3'-carboxylate (3I). White solid; Yield $125 \mathrm{mg}$, 99\%; 9:1 dr; Major isomer (isolated by fractional crystallization): $\mathrm{mp}$ 199-200 ${ }^{\circ} \mathrm{C}$; IR (KBr film) 3030 (w), 2926 (w), 1740 (s), 1711 (vs), 1610

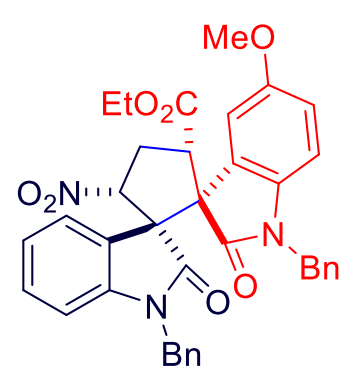

(m), $1548(\mathrm{~s}), 1493(\mathrm{~s}), 1468(\mathrm{~m}), 1456(\mathrm{~m}), 1435(\mathrm{w}), 1370(\mathrm{~s}), 1205(\mathrm{~s}), 1184$ $(\mathrm{m}), 120(\mathrm{w}), 755(\mathrm{~s}) \mathrm{cm}^{-1} ;{ }^{1} \mathrm{H} \mathrm{NMR}\left(400 \mathrm{MHz}, \mathrm{CDCl}_{3}\right) \delta 0.71(\mathrm{t}, J=7.2 \mathrm{~Hz}$, $3 \mathrm{H}$ ), 3.02 (ddd collapsed to dt, $J=12.3,7.7 \mathrm{~Hz}, 1 \mathrm{H}), 3.49$ (s, 3H), 3.69, 3.85 (ABqq, $J=10.8,7.2 \mathrm{~Hz}, 2 \mathrm{H}$ ), 3.88 (overlapped dd, $J=12.3,7.7 \mathrm{~Hz}, 1 \mathrm{H}$ ), 4.29 $(\mathrm{q}, J=12.3 \mathrm{~Hz}, 1 \mathrm{H}), 4.64,4.84(\mathrm{ABq}, J=16.0 \mathrm{~Hz}, 2 \mathrm{H}), 4.68,4.91(\mathrm{ABq}, J=$ $16.1 \mathrm{~Hz}, 2 \mathrm{H}), 5.97(\mathrm{dd}, J=12.3,7.7 \mathrm{~Hz}, 1 \mathrm{H}), 6.30(\mathrm{~d}, J=8.6 \mathrm{~Hz}, 1 \mathrm{H}), 6.43$ $(\mathrm{d}, J=7.7 \mathrm{~Hz}, 1 \mathrm{H}), 6.61(\mathrm{dd}, J=8.6,2.6 \mathrm{~Hz}, 1 \mathrm{H}), 6.76-6.82(\mathrm{~m}, 4 \mathrm{H}), 6.88(\mathrm{t}$, $J=7.7 \mathrm{~Hz}, 1 \mathrm{H}), 6.99(\mathrm{~d}, J=2.6 \mathrm{~Hz}, 1 \mathrm{H}), 7.08-7.20(\mathrm{~m}, 7 \mathrm{H}), 7.49(\mathrm{~d}, J=7.7$ $\mathrm{Hz}, 1 \mathrm{H}) ;{ }^{13} \mathrm{C}\{\mathrm{H}\}$ NMR $\left(125 \mathrm{MHz}, \mathrm{CDCl}_{3}\right)$ 13.7, 31.0, 43.9, 44.0, 47.8, 55.8, 60.6, 60.7, 61.0, 86.8, $109.7,110.1,113.0,115.3,123.1,124.2,125.1,126.0,126.7(\times 2), 127.4,127.6,128.8(\times 2), 129.7$, 134.7, 135.1, 136.6, 144.2, 156.1, 169.3, 173.8, 177.9; HRMS (ESI) m/z: $\left[\mathrm{M}+\mathrm{Na}^{+}\right.$Calcd for $\mathrm{C}_{37} \mathrm{H}_{33} \mathrm{~N}_{3} \mathrm{O}_{7} \mathrm{Na}$ 654.2211; Found 654.2215. 
Ethyl 1,1"'-dibenzyl-5",7"'-dimethyl-5'-nitro-2,2"'-dioxodispiro[indoline-3,1'-cyclopentane-2',3"indoline]-3'-carboxylate (3m). White solid; Yield $88 \mathrm{mg}, 70 \%$; 8:1 dr; Major isomer (isolated by fractional crystallization): $\mathrm{mp} 208-210{ }^{\circ} \mathrm{C}$; IR (KBr film) 3027 (w), 2927 (w), 1742 (s), 1711 (vs), 1610

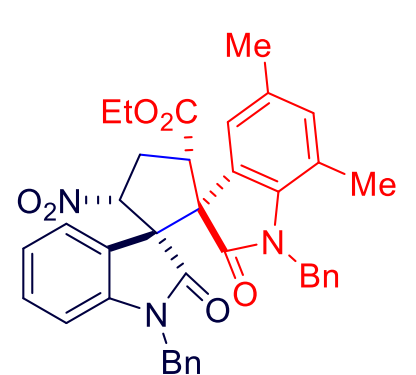

(m), 1548 (s), $1490(\mathrm{~m}), 1468(\mathrm{~m}), 1454(\mathrm{~m}), 1369(\mathrm{~s}), 1204(\mathrm{~m}), 1174(\mathrm{~m})$, $756(\mathrm{~s}) \mathrm{cm}^{-1} ;{ }^{1} \mathrm{H}$ NMR $\left(400 \mathrm{MHz}, \mathrm{CDCl}_{3}\right) \delta 0.77(\mathrm{t}, J=7.2 \mathrm{~Hz}, 3 \mathrm{H}), 1.99$ $(\mathrm{s}, 3 \mathrm{H}), 2.04(\mathrm{~s}, 3 \mathrm{H}), 3.00$ (ddd collapsed to dt, $J=12.3,7.7 \mathrm{~Hz}, 1 \mathrm{H}), 3.72$ $(\mathrm{dq}, J=10.8,7.2, \mathrm{~Hz}, 1 \mathrm{H}), 3.85(\mathrm{dd}, J=12.3,7.7 \mathrm{~Hz}, 1 \mathrm{H}), 3.90(\mathrm{dq}, J=$ $10.8,7.2, \mathrm{~Hz}, 1 \mathrm{H}), 4.30(\mathrm{q}, J=12.3 \mathrm{~Hz}, 1 \mathrm{H}), 4.61(\mathrm{~d}, J=16.1 \mathrm{~Hz}, 1 \mathrm{H})$, $4.93(2 \times$ overlapped d, expected $J=16.1$ and $17.1 \mathrm{~Hz}$, observed $J=16.6$ $\mathrm{Hz}, 2 \mathrm{H}), 5.10(\mathrm{~d}, J=17.1 \mathrm{~Hz}, 1 \mathrm{H}), 6.00(\mathrm{dd}, J=12.3,7.7 \mathrm{~Hz}, 1 \mathrm{H}), 6.42$ $(\mathrm{d}, J=7.7 \mathrm{~Hz}, 1 \mathrm{H}), 6.69(\mathrm{~s}, 1 \mathrm{H}), 6.78-6.88(\mathrm{~m}, 5 \mathrm{H}), 7.08$ (overlapped s, 1H), 7.10 (overlapped t, $J=7.7 \mathrm{~Hz}, 1 \mathrm{H}), 7.12-7.20(\mathrm{~m}, 6 \mathrm{H}), 7.43(\mathrm{~d}, J=7.7 \mathrm{~Hz}, 1 \mathrm{H}) ;{ }^{13} \mathrm{C}\{\mathrm{H}\} \mathrm{NMR}$ $\left(100 \mathrm{MHz} \mathrm{CDCl}_{3}\right)$ 13.7, 18.6, 20.8, 30.9, 43.8, 45.2, 48.1, 59.9, 60.7, 60.9, 86.7, 109.6, 119.5, 123.0, $124.3,125.0,125.1,125.3,125.6,126.7,127.2,127.4,128.7,128.9,129.7,132.7,134.1,135.2,136.8$, 138.9, 144.2, 169.4, 173.9, 179.1; HRMS (ESI) m/z: $[\mathrm{M}+\mathrm{K}]^{+}$Calcd for $\mathrm{C}_{38} \mathrm{H}_{35} \mathrm{~N}_{3} \mathrm{O}_{6} \mathrm{~K} 668.2157$; Found 668.2153; Selected X-ray, Data (CCDC 2035368): $\mathrm{C}_{38} \mathrm{H}_{35} \mathrm{~N}_{3} \mathrm{O}_{6}, M$ 629.25, Monoclinic, space group $\mathrm{P}$

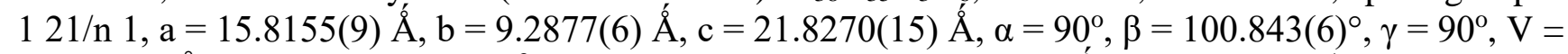
3148.9(3) ̊3 $, \mathrm{Dc}=1.328 \mathrm{Mg} / \mathrm{m}^{3} \mathrm{Z}=4, \mathrm{~F}(000)=1328, \lambda=0.71073 \AA \AA, \mu=1.903 \mathrm{~mm}^{-1}$, Total/ unique reflections $=13984 / 5515[\mathrm{R}(\mathrm{int})=0.0569], \mathrm{T}=293(2) \mathrm{K}, \theta$ range $=2.56$ to $25.00^{\circ}$, Final $\mathrm{R}[\mathrm{I}>2 \sigma(\mathrm{I})]$ : $\mathrm{R} 1=0.0522, \mathrm{wR} 2=0.1089, \mathrm{R}$ (all data): $\mathrm{R} 1=0.0838, \mathrm{wR} 2=0.1292$.

Ethyl 1,1"'-dibenzyl-5"'-fluoro-5'-nitro-2,2"'-dioxodispiro[indoline-3,1'-cyclopentane-2',3"'indoline]-3'-carboxylate (3n). White solid; Yield $120 \mathrm{mg}$, 99\%; 8:1 dr; Major isomer (isolated by fractional crystallization): $\mathrm{mp} 125-127{ }^{\circ} \mathrm{C}$; IR (KBr film) 3030 (vw), 1740 (s), 1713 (vs), 1612 (m),

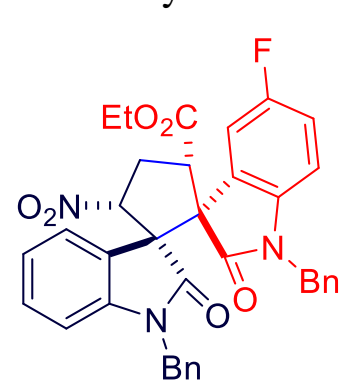
1549 (s), 1491 (s), 1469 (m), 1454 (m), 1370 (s), 1181 (m), 756 (s) $\mathrm{cm}^{-1} ;{ }^{1} \mathrm{H}$ NMR $\left(500 \mathrm{MHz}, \mathrm{CDCl}_{3}\right) \delta 0.78(\mathrm{t}, J=7.1 \mathrm{~Hz}, 3 \mathrm{H}), 3.04$ (ddd collapsed to dt, $J=12.3,7.9 \mathrm{~Hz}, 1 \mathrm{H}), 3.78(\mathrm{dq}, J=10.9,7.1 \mathrm{~Hz}, 1 \mathrm{H}), 3.84(\mathrm{dd}, J=12.3,7.9$ $\mathrm{Hz}, 1 \mathrm{H}), 3.91(\mathrm{dq}, J=10.9,7.1 \mathrm{~Hz}, 1 \mathrm{H}), 4.29(\mathrm{q}, J=12.3 \mathrm{~Hz}, 1 \mathrm{H}), 4.69,4.80$ $(\mathrm{ABq}, J=16.0 \mathrm{~Hz}, 1 \mathrm{H}), 4.73,4.85(\mathrm{ABq}, J=16.1 \mathrm{~Hz}, 1 \mathrm{H}), 5.98(\mathrm{dd}, J=12.3$, $7.9 \mathrm{~Hz}, 1 \mathrm{H}), 6.32(\mathrm{dd}, J=8.6,4.3 \mathrm{~Hz}, 1 \mathrm{H}), 6.48(\mathrm{~d}, J=7.7 \mathrm{~Hz}, 1 \mathrm{H}), 6.75-6.80$ $(\mathrm{m}, 3 \mathrm{H}), 6.88(\mathrm{t}, J=7.7 \mathrm{~Hz}, 1 \mathrm{H}), 6.93(\mathrm{~d}, J=7.6 \mathrm{~Hz}, 2 \mathrm{H}), 7.10-7.21(\mathrm{~m}, 8 \mathrm{H})$, $7.44(\mathrm{~d}, J=7.7 \mathrm{~Hz}, 1 \mathrm{H}) ;{ }^{13} \mathrm{C}\{\mathrm{H}\} \mathrm{NMR}\left(125 \mathrm{MHz}, \mathrm{CDCl}_{3}\right)$ 13.7, 30.9, 44.0, 44.1 ( $\times 2), 47.7,60.5,61.2,86.6,109.8,110.0(\mathrm{~d}, J=7.5 \mathrm{~Hz}), 115.0(\mathrm{~d}, J=26.2 \mathrm{~Hz}), 115.9(\mathrm{~d}, J=23.7$ $\mathrm{Hz}), 123.2,123.8,125.0,126.5(\mathrm{~d}, J=8.7 \mathrm{~Hz}), 126.7,127.0,127.6,127.8,128.8,128.9,129.9,134.3$, 135.0, $139.3(\mathrm{~d}, J=1.2 \mathrm{~Hz}), 144.1,159.1(\mathrm{~d}, J=240.0 \mathrm{~Hz}), 169.0,173.7,177.9 ;{ }^{19} \mathrm{~F}$ NMR $(470 \mathrm{MHz}$, $\mathrm{CDCl}_{3}$ ) -118.94; HRMS (ESI) m/z: $[\mathrm{M}+\mathrm{H}]^{+}$Calcd for $\mathrm{C}_{36} \mathrm{H}_{31} \mathrm{~N}_{3} \mathrm{O}_{6} \mathrm{~F}$ 620.2191; Found 620.2196.

Ethyl 1,1'-dibenzyl-5"'-chloro-5'-nitro-2,2"'-dioxodispiro[indoline-3,1'-cyclopentane-2',3"'indoline]-3'-carboxylate (3o). White solid; Yield $78 \mathrm{mg}, 61 \%$; 7:1 dr; Major isomer (isolated by fractional crystallization): $\mathrm{mp} 161-163{ }^{\circ} \mathrm{C}$; IR (KBr film) 2919 (m), 2850 (w), 1729 (vs), 1715 (vs),

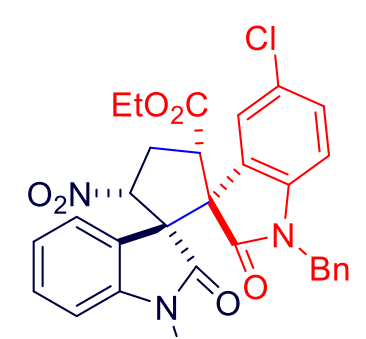

$\mathrm{Bn}$ $1610(\mathrm{~m}), 1550(\mathrm{~m}), 1487$ (s), $1469(\mathrm{~m}), 1455(\mathrm{~m}), 1368(\mathrm{~s}), 1205(\mathrm{~m}), 1177$ (m), $755(\mathrm{~s}) \mathrm{cm}^{-1} ;{ }^{1} \mathrm{H}$ NMR $\left(500 \mathrm{MHz}, \mathrm{CDCl}_{3}\right) \delta 0.80(\mathrm{t}, J=7.1 \mathrm{~Hz}, 3 \mathrm{H}), 3.05$ (ddd collapsed to dt, $J=12.4,7.8 \mathrm{~Hz}, 1 \mathrm{H}$ ), 3.78-3.86 (unresolved $\mathrm{m}, 2 \mathrm{H}$ ), 3.92 $(\mathrm{dq}, J=10.8,7.1 \mathrm{~Hz}, 1 \mathrm{H}), 4.29(\mathrm{q}, J=12.4 \mathrm{~Hz}, 1 \mathrm{H}), 4.70,4.81(\mathrm{ABq}, J=15.9$ $\mathrm{Hz}, 1 \mathrm{H}), 4.74,4.83(\mathrm{ABq}, J=16.2 \mathrm{~Hz}, 1 \mathrm{H}), 5.99(\mathrm{dd}, J=12.4,7.8 \mathrm{~Hz}, 1 \mathrm{H})$, $6.32(\mathrm{~d}, J=8.4 \mathrm{~Hz}, 1 \mathrm{H}), 6.47(\mathrm{~d}, J=7.7 \mathrm{~Hz}, 1 \mathrm{H}), 6.78(\mathrm{~d}, J=7.6 \mathrm{~Hz}, 2 \mathrm{H})$, $6.87(\mathrm{t}, J=7.7 \mathrm{~Hz}, 1 \mathrm{H}), 6.94-6.97(\mathrm{~m}, 2 \mathrm{H}), 7.06(\mathrm{dd}, J=8.4,2.1 \mathrm{~Hz}, 1 \mathrm{H}), 7.11$ $(\mathrm{t}, J=7.7 \mathrm{~Hz}, 1 \mathrm{H}), 7.13-7.22(\mathrm{~m}, 6 \mathrm{H}), 7.38(\mathrm{~d}, J=2.1 \mathrm{~Hz}, 1 \mathrm{H}), 7.41(\mathrm{~d}, J=$ $7.7 \mathrm{~Hz}, 1 \mathrm{H}) ;{ }^{13} \mathrm{C}\{\mathrm{H}\} \mathrm{NMR}\left(125 \mathrm{MHz}, \mathrm{CDCl}_{3}\right) 13.8,30.9,44.1(\times 2), 47.7,60.4,60.5,61.3,86.6,109.9$, $110.5,123.2$, 123.7, 124.9, 126.6, 126.7, 127.0, 127.2, 127.6, 127.8, 128.7, 128.9, 129.0, 129.5, 129.9, 
134.2, 135.0, 141.9, 144.1, 169.0, 173.7, 177.8; HRMS (ESI) m/z: $\left[\mathrm{M}+\mathrm{Na}^{+} \mathrm{Calcd}_{\text {for }}\right.$ $\mathrm{C}_{36} \mathrm{H}_{30} \mathrm{~N}_{3} \mathrm{O}_{6} \mathrm{ClNa}$ 658.1715; Found 658.1715.

Ethyl 1,1'-dibenzyl-5',5''-dinitro-2,2"'-dioxodispiro[indoline-3,1'-cyclopentane-2',3''-indoline]3'-carboxylate (3p). Light yellow solid; Yield $93 \mathrm{mg}$, 72\%; 1.6:1 dr; Major isomer (isolated by chromatography): mp 175-177 ${ }^{\circ} \mathrm{C}$; IR (KBr film) 3027 (w), 2935 (w), 1717 (vvs), 1614 (s), 1549 (s),

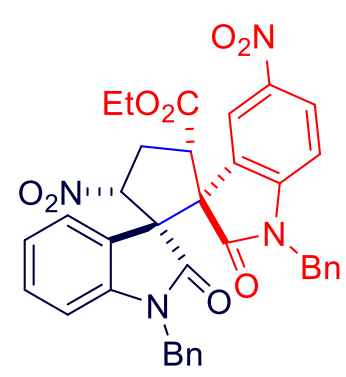

$1523(\mathrm{~m}), 1491(\mathrm{~m}), 1469(\mathrm{w}), 1369(\mathrm{~s}), 1337(\mathrm{vs}), 1220(\mathrm{~m}), 1175(\mathrm{w}), 1080$ (w), $756(\mathrm{vs}) \mathrm{cm}^{-1} ;{ }^{1} \mathrm{H}$ NMR $\left(500 \mathrm{MHz}, \mathrm{CDCl}_{3}\right) \delta 1.15(\mathrm{t}, J=7.1 \mathrm{~Hz}, 3 \mathrm{H})$, 3.47-3.60 (m, 2H), 4.04, 4.10 (ABqq, $J=10.8,7.1 \mathrm{~Hz}, 2 \mathrm{H}), 4.62,5.09(2 \times \mathrm{d}$, $J=15.9 \mathrm{~Hz}, 2 \mathrm{H}), 4.78(\mathrm{dd}, J=12.0,8.4 \mathrm{~Hz}, 1 \mathrm{H}), 4.87,4.92(\mathrm{ABq}, J=15.9$ $\mathrm{Hz}, 2 \mathrm{H}), 6.20$ (dd, $J=11.1,7.2 \mathrm{~Hz}, 1 \mathrm{H}), 6.54$ (overlapped d, $J=7.7 \mathrm{~Hz}, 1 \mathrm{H}$ ), 6.55 (overlapped d, $J=8.7 \mathrm{~Hz}, 1 \mathrm{H}), 6.86(\mathrm{t}, J=7.7 \mathrm{~Hz}, 1 \mathrm{H}), 7.12-7.15(\mathrm{~m}$, $5 \mathrm{H}), 7.24-7.32(\mathrm{~m}, 7 \mathrm{H}), 8.05(\mathrm{dd}, J=8.7,2.2 \mathrm{~Hz}, 1 \mathrm{H}), 8.22(\mathrm{~d}, J=2.2 \mathrm{~Hz}$, $1 \mathrm{H})$; Confirmed by ${ }^{1} \mathrm{H}-{ }^{1} \mathrm{H}$ COSY, ${ }^{1} \mathrm{H}-{ }^{1} \mathrm{H}$ NOESY and ${ }^{1} \mathrm{H}-{ }^{13} \mathrm{C}$ HSQC experiments; ${ }^{13} \mathrm{C}\{\mathrm{H}\}$ NMR $\left(125 \mathrm{MHz}, \mathrm{CDCl}_{3}\right)$ 14.1, 29.5, 44.4, 44.6, 47.0, $61.1,61.2,61.9,86.1,109.2,110.3,120.0,123.3,123.4,123.6,126.0,126.6,127.2,127.4,128.0,128.2$, $128.9,129.1,130.1(\times 2), 134.0,134.7,143.6,149.5,170.7,174.3,177.4 ; \mathrm{HRMS}(\mathrm{ESI}) \mathrm{m} / \mathrm{z}:[\mathrm{M}+\mathrm{H}]^{+}$ Calcd for $\mathrm{C}_{36} \mathrm{H}_{31} \mathrm{~N}_{4} \mathrm{O}_{8}$ 647.2136; Found 647.2135.

Ethyl 1-benzyl-5'-nitro-2,2"'-dioxo-1"'-(prop-2-yn-1-yl)dispiro[indoline-3,1'-cyclopentane-2',3"indoline]-3'-carboxylate (3q). White solid; Yield $108 \mathrm{mg}$, 98\%; 4:1 dr; Major isomer (isolated by fractional crystallization): $\mathrm{mp}$ 145.5-147.5 ${ }^{\circ} \mathrm{C}$; IR (KBr film) 3295 (w), 3029 (w), 2983 (w), 1740 (s),

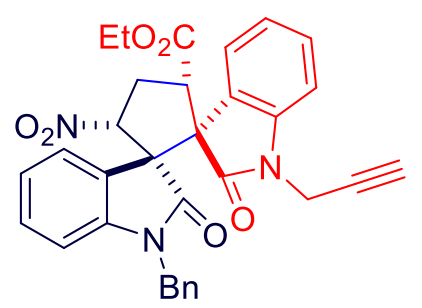

1713 (vs), 1612 (m), 1548 (s), 1489 (m), 1468 (m), 1370 (s), 1208 (w), 1183 (w), 1105 (w), 1017 (w), 755 (s) cm ${ }^{-1} ;{ }^{1} \mathrm{H}$ NMR (500 MHz, $\mathrm{CDCl}_{3}$ ) $\delta 0.76(\mathrm{t}, J=7.1 \mathrm{~Hz}, 3 \mathrm{H}), 2.06(\mathrm{t}, J=2.4 \mathrm{~Hz}, 1 \mathrm{H}), 3.03$ (ddd collapsed to $\mathrm{dt}, J=12.4,8.0 \mathrm{~Hz}, 1 \mathrm{H}), 3.70-3.76(\mathrm{~m}, 2 \mathrm{H}), 3.88(\mathrm{dq}, J=10.7,7.1 \mathrm{~Hz}$, $1 \mathrm{H}), 4.26$ (overlapped q, $J=12.4 \mathrm{~Hz}, 1 \mathrm{H}), 4.27,4.39(\mathrm{ABqd}, J=17.5,2.4$ $\mathrm{Hz}, 2 \mathrm{H}), 4.67,4.73(\mathrm{ABq}, J=16.0 \mathrm{~Hz}, 2 \mathrm{H}), 5.97(\mathrm{dd}, J=12.4,8.0 \mathrm{~Hz}$, $1 \mathrm{H}), 6.36(\mathrm{~d}, J=7.7 \mathrm{~Hz}, 1 \mathrm{H}), 6.80(\mathrm{~d}, J=7.8 \mathrm{~Hz}, 1 \mathrm{H}), 6.87(\mathrm{t}, J=7.7 \mathrm{~Hz}$, $1 \mathrm{H}), 6.90-6.94(\mathrm{~m}, 3 \mathrm{H}), 7.03(\mathrm{t}, J=7.7 \mathrm{~Hz}, 1 \mathrm{H}), 7.18-7.25(\mathrm{~m}, 4 \mathrm{H}), 7.28(\mathrm{~d}, J=7.8 \mathrm{~Hz}, 1 \mathrm{H}), 7.38(\mathrm{~d}, J$ $=7.7 \mathrm{~Hz}, 1 \mathrm{H}) ;{ }^{13} \mathrm{C}\{\mathrm{H}\} \mathrm{NMR}\left(125 \mathrm{MHz}, \mathrm{CDCl}_{3}\right)$ 13.7, 29.6, 30.8, 44.0, 46.8, 60.5, 60.6, 61.0, 72.7, 76.0, 86.4, 109.1, 109.5, 123.0, 123.3, 123.5, $124.4(\times 2), 126.7,127.2,127.5,128.8,129.5,129.7$, 135.1, 142.0, 143.8, 169.3, 173.7, 177.3; HRMS (ESI) m/z: $[\mathrm{M}+\mathrm{H}]^{+}$Calcd for $\mathrm{C}_{32} \mathrm{H}_{28} \mathrm{~N}_{3} \mathrm{O}_{6} 550.1973$; Found 550.1975.

Ethyl 1'-allyl-1-benzyl-5'-nitro-2,2"'-dioxodispiro[indoline-3,1'-cyclopentane-2',3''-indoline]-3'carboxylate (3r). White solid; Yield $108 \mathrm{mg}$, 98\%; 6:1 dr; Major isomer (isolated by fractional crystallization): $\mathrm{mp} 135-137{ }^{\circ} \mathrm{C}$; IR (KBr film) 3022 (m), 2983 (m), 1740 (vs), 1713 (vs), 1611 (s), 1548

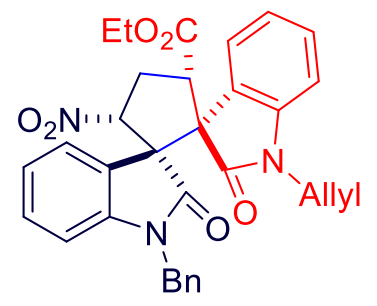
(s), $1490(\mathrm{~m}), 1468(\mathrm{~m}), 1371(\mathrm{vs}), 1206(\mathrm{~m}), 1183(\mathrm{~m}), 1104(\mathrm{w}), 932(\mathrm{w})$, 756 (vs) cm ${ }^{-1} ;{ }^{1} \mathrm{H}$ NMR $\left(500 \mathrm{MHz}, \mathrm{CDCl}_{3}\right) \delta 0.72(\mathrm{t}, J=7.1 \mathrm{~Hz}, 3 \mathrm{H}), 3.01$ (ddd collapsed to dt, $J=12.4,7.9 \mathrm{~Hz}, 1 \mathrm{H}), 3.69(\mathrm{dq}, J=10.7,7.1 \mathrm{~Hz}, 1 \mathrm{H}$ ), $3.76(\mathrm{dd}, J=12.4,7.9 \mathrm{~Hz}, 1 \mathrm{H}), 3.86(\mathrm{dq}, J=10.7,7.1 \mathrm{~Hz}, 1 \mathrm{H}), 4.05(\mathrm{ddt}, J$ $=16.7,5.0,1.6 \mathrm{~Hz}, 1 \mathrm{H}), 4.29$ (overlapped q, $J=12.4 \mathrm{~Hz}, 1 \mathrm{H}$ ), 4.28-4.33 (overlapped m, 1H), 4.69, 4.73 (overlapped ABq, $J=16.0 \mathrm{~Hz}, 2 \mathrm{H}$ ), 4.70-4.75 (overlapped $\mathrm{m}, 1 \mathrm{H}), 4.98(\mathrm{~d}, J=10.4 \mathrm{~Hz}, 1 \mathrm{H}), 5.49(\mathrm{ddd}, J=15.3,10.4,5.0$ $\mathrm{Hz}, 1 \mathrm{H}), 5.97(\mathrm{dd}, J=12.4,7.9 \mathrm{~Hz}, 1 \mathrm{H}), 6.37(\mathrm{~d}, J=7.6 \mathrm{~Hz}, 1 \mathrm{H}), 6.59(\mathrm{~d}, J=7.8 \mathrm{~Hz}, 1 \mathrm{H}), 6.83(\mathrm{t}, J=$ $7.6 \mathrm{~Hz}, 1 \mathrm{H}), 6.87-6.92(\mathrm{~m}, 3 \mathrm{H}), 7.04(\mathrm{t}, J=7.6 \mathrm{~Hz}, 1 \mathrm{H}), 7.14-7.20(\mathrm{~m}, 4 \mathrm{H}), 7.29(\mathrm{~d}, J=7.8 \mathrm{~Hz}, 1 \mathrm{H})$, $7.42(\mathrm{~d}, J=7.6 \mathrm{~Hz}, 1 \mathrm{H}) ;{ }^{13} \mathrm{C}\{\mathrm{H}\} \mathrm{NMR}\left(125 \mathrm{MHz} \mathrm{CDCl}_{3}\right) 13.7,30.9,42.4,43.9,47.2,60.4,60.5,60.9$, 86.5, 109.2, 109.6, 117.5, 122.8, 123.0, 123.9, 124.7, 124.8, 126.6, 127.1, 127.5, 128.7, 129.5, 129.7, 
130.3, 135.1, 143.3, 144.0, 169.4, 173.8, 177.8; HRMS (ESI) m/z: $[\mathrm{M}+\mathrm{H}]^{+}$Calcd for $\mathrm{C}_{32} \mathrm{H}_{30} \mathrm{~N}_{3} \mathrm{O}_{6}$ 552.2129; Found 552.2126.

Ethyl 1-benzyl-1"'-methyl-5'-nitro-2,2"'-dioxodispiro[indoline-3,1'-cyclopentane-2',3"'-indoline]3'-carboxylate (3s). White solid; Yield $103 \mathrm{mg}$, 98\%; 3:1 dr; Major isomer (77 mg, 73\% isolated by fractional crystallization): mp 185-187 ${ }^{\circ} \mathrm{C}$; IR (KBr film) 3057 (w), 3025 (m), 2979 (m), 2961 (m), 2932

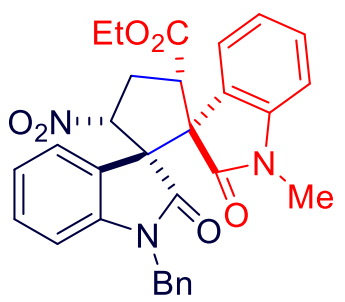
(m), 1716 (vs), 1611 (s), 1548 (s), 1492 (s), 1469 (s), 1455 (m), 1372 (vs), 1207 (m), 1176 (m), 1097 (w), 754 (vs) cm ${ }^{-1} ;{ }^{1} \mathrm{H}$ NMR (500 MHz, $\left.\mathrm{CDCl}_{3}\right) \delta$ $0.78(\mathrm{t}, J=7.1 \mathrm{~Hz}, 3 \mathrm{H}), 3.00-3.06$ (overlapped $\mathrm{m}, 1 \mathrm{H}), 3.02$ (overlapped s, $3 \mathrm{H}), 3.72(\mathrm{dd}, J=12.3,7.5 \mathrm{~Hz}, 1 \mathrm{H}), 3.71(\mathrm{dq}, J=10.8,7.5 \mathrm{~Hz}, 1 \mathrm{H}), 3.91(\mathrm{dq}$, $J=10.8,7.1 \mathrm{~Hz}, 1 \mathrm{H}), 4.29(\mathrm{q}, J=12.3 \mathrm{~Hz}, 1 \mathrm{H}), 4.67,4.74(\mathrm{ABq}, J=16.0 \mathrm{~Hz}$, $2 \mathrm{H}), 6.01(\mathrm{dd}, J=12.3,7.5 \mathrm{~Hz}, 1 \mathrm{H}), 6.36(\mathrm{~d}, J=7.6 \mathrm{~Hz}, 1 \mathrm{H}), 6.59(\mathrm{~d}, J=7.8$ $\mathrm{Hz}, 1 \mathrm{H}), 6.84(\mathrm{t}, J=7.6 \mathrm{~Hz}, 1 \mathrm{H}), 6.89-6.92(\mathrm{~m}, 3 \mathrm{H}), 7.04(\mathrm{t}, J=7.6 \mathrm{~Hz}, 1 \mathrm{H})$, 7.18-7.21 (m, 4H), $7.24(\mathrm{~d}, J=7.8 \mathrm{~Hz}, 1 \mathrm{H}), 7.39(\mathrm{~d}, J=7.6 \mathrm{~Hz}, 1 \mathrm{H})$; Confirmed by ${ }^{1} \mathrm{H}-{ }^{1} \mathrm{H}$ COSY and ${ }^{1} \mathrm{H}-{ }^{1} \mathrm{H}$ NOESY experiments; ${ }^{13} \mathrm{C}\{\mathrm{H}\}$ NMR $\left(100 \mathrm{MHz}, \mathrm{CDCl}_{3}\right)$ 13.7, 26.4, 30.8, 43.9, 46.5, 60.5, 60.6, 61.0, 86.3, 108.2, 109.5, 122.5, 122.9, 123.6, 124.1, 124.3, 126.4, 127.1, 127.5, 128.7, 129.5, 129.7, 135.1, 143.8, 143.9, 169.5, 173.7, 178.0; HRMS (ESI) m/z: $[\mathrm{M}+\mathrm{H}]^{+}$Calcd for $\mathrm{C}_{30} \mathrm{H}_{28} \mathrm{~N}_{3} \mathrm{O}_{6}$ 526.1973; Found 526.1970.

Ethyl 1-benzyl-1"-methyl-5'-nitro-2,2"'-dioxodispiro[indoline-3,1'-cyclopentane-2',3''-indoline]3'-carboxylate (3'). Minor isomer (isolated from the mother liquor after fractional crystallization of

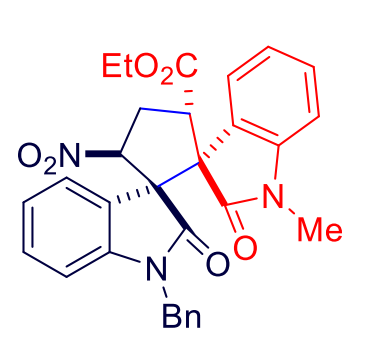
the major isomer); Compound isolated using silica gel column chromatography by eluting with $72 / 25$ petroleum ether/ethyl acetate; White solid; Yield $26 \mathrm{mg}, 25 \%$; mp 191-192 ${ }^{\circ} \mathrm{C}$; IR (KBr film) 3027 (vw), 2941 (vw), 1722 (s), 1612 (w), $1551(\mathrm{~m}), 1490$ (w), 1469 (w), 1373 (w), $1218(\mathrm{~m})$, $1042(\mathrm{~m}), 755(\mathrm{vs}) \mathrm{cm}^{-1} ;{ }^{1} \mathrm{H}$ NMR $\left(500 \mathrm{MHz}, \mathrm{CDCl}_{3}\right) \delta 0.64(\mathrm{t}, J=7.1 \mathrm{~Hz}$, $3 \mathrm{H}), 2.89(\mathrm{~s}, 3 \mathrm{H}), 3.57(\mathrm{t}, J=9.7 \mathrm{~Hz}, 2 \mathrm{H}), 3.66(\mathrm{dq}, J=10.8,7.1 \mathrm{~Hz}, 1 \mathrm{H})$, $3.80(\mathrm{dq}, J=10.8,7.1 \mathrm{~Hz}, 1 \mathrm{H}), 4.77(\mathrm{t}, J=9.7 \mathrm{~Hz}, 1 \mathrm{H}), 4.95(\mathrm{ABq}, J=15.8$ $\mathrm{Hz}, 2 \mathrm{H}), 5.59(\mathrm{t}, J=9.7 \mathrm{~Hz}, 1 \mathrm{H}), 5.95(\mathrm{~d}, J=7.5 \mathrm{~Hz}, 1 \mathrm{H}), 6.51(\mathrm{t}, J=7.5 \mathrm{~Hz}$, $1 \mathrm{H}), 6.55(\mathrm{~d}, J=7.5 \mathrm{~Hz}, 1 \mathrm{H}), 6.67(\mathrm{~d}, J=7.7 \mathrm{~Hz}, 1 \mathrm{H}), 7.00(\mathrm{t}, J=7.5 \mathrm{~Hz}, 1 \mathrm{H}), 7.17(\mathrm{t}, J=7.7 \mathrm{~Hz}, 1 \mathrm{H})$, $7.25(\mathrm{t}, J=7.7 \mathrm{~Hz}, 1 \mathrm{H}), 7.32-7.38(\mathrm{~m}, 4 \mathrm{H}), 7.39-7.43(\mathrm{~m}, 2 \mathrm{H})$; Confirmed by ${ }^{1} \mathrm{H}-{ }^{1} \mathrm{H}$ COSY and ${ }^{1} \mathrm{H}-{ }^{1} \mathrm{H}$ NOESY experiments; ${ }^{13} \mathrm{C}\{\mathrm{H}\}$ NMR $\left(125 \mathrm{MHz}, \mathrm{CDCl}_{3}\right)$ 13.5, 26.3, 28.8, 44.5, 44.9, 60.7, 62.0, 62.3, 86.8, 108.6, 109.5, 121.6, 122.5, 122.7, 123.0, 124.0, 126.6, 127.5, 127.6, 128.7, 129.5, 130.1, 135.5, 143.8, 144.5, 170.2, 172.3, 173.4; HRMS (ESI) m/z: $[\mathrm{M}+\mathrm{H}]^{+}$Calcd for $\mathrm{C}_{30} \mathrm{H}_{28} \mathrm{~N}_{3} \mathrm{O}_{6}$ 526.1973; Found 526.1975. Selected X-ray Data (CCDC 2034424): $\mathrm{C}_{30} \mathrm{H}_{27} \mathrm{~N}_{3} \mathrm{O}_{6}, M$ 525.57, Orthorhombic, space group

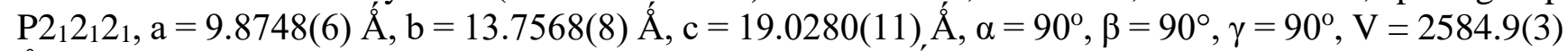
$\AA^{3}, \rho=1.3504 \mathrm{~g} / \mathrm{cm}^{3}, \mathrm{Z}=4, \mathrm{~F}(000)=1104.6, \lambda=0.71073 \AA \AA, \mu=0.095 \mathrm{~mm}^{-1}$, Total/ unique reflections $=19191 / 4536[\mathrm{R}($ int $)=0.0742], \mathrm{T}=150 \mathrm{~K}, \theta$ range $=4.64$ to $50.00^{\circ}$, Final $\mathrm{R}[\mathrm{I}>2 \sigma(\mathrm{I})]: \mathrm{R}_{1}=0.0511$, $\mathrm{wR}_{2}=0.1289, \mathrm{R}$ (all data): $\mathrm{R}_{1}=0.0711, \mathrm{wR}_{2}=0.1561$.

1"'-Benzyl-3'-nitro-5' -(p-tolyl)dispiro[indene-2,1'-cyclopentane-2',3"'-indoline]-1,2",3-trione (4).

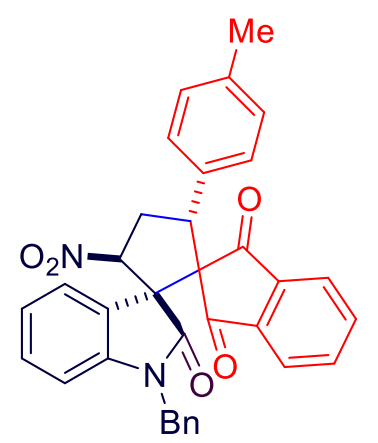

White solid; Yield $46 \mathrm{mg}, 42 \%$; Single isomer (compound isolated using silica gel column chromatography by eluting with $82 / 18$ petroleum ether/ethyl acetate); $\mathrm{mp} 240-241.5^{\circ} \mathrm{C}$; IR (KBr film) 3030 (vw), 2922 (s), $2851(\mathrm{~m}), 1719$ (vs), $1611(\mathrm{~m}), 1549$ (s), $1370(\mathrm{~s}), 1354(\mathrm{~m}), 1260(\mathrm{~m}), 759$ (s) $\mathrm{cm}^{-1} ;{ }^{1} \mathrm{H}$ NMR $\left(500 \mathrm{MHz}, \mathrm{CDCl}_{3}\right) \delta 2.10(\mathrm{~s}, 3 \mathrm{H}), 3.40-3.47(\mathrm{~m}, 1 \mathrm{H})$, $3.50-3.57(\mathrm{~m}, 1 \mathrm{H}), 4.70,5.09(\mathrm{ABq}, J=15.8 \mathrm{~Hz}, 2 \mathrm{H}), 5.14(\mathrm{t}, J=9.9 \mathrm{~Hz}$, $1 \mathrm{H}), 6.33(\mathrm{dd}, J=11.3,6.3 \mathrm{~Hz}, 1 \mathrm{H}), 6.54(\mathrm{~d}, J=7.6 \mathrm{~Hz}, 1 \mathrm{H}), 6.73(\mathrm{t}, J=$ $7.6 \mathrm{~Hz}, 1 \mathrm{H}), 6.84(\mathrm{~d}, J=7.9 \mathrm{~Hz}, 2 \mathrm{H}), 6.98$ (overlapped t, $J=7.6 \mathrm{~Hz}, 1 \mathrm{H}$ ), 6.99 (overlapped d, $J=7.9 \mathrm{~Hz}, 2 \mathrm{H}), 7.10(\mathrm{~d}, J=7.6 \mathrm{~Hz}, 1 \mathrm{H}), 7.28(\mathrm{~d}, J=$ 
$7.5 \mathrm{~Hz}, 1 \mathrm{H}), 7.36(\mathrm{t}, J=7.5 \mathrm{~Hz}, 2 \mathrm{H}), 7.43(\mathrm{~d}, J=7.5 \mathrm{~Hz}, 2 \mathrm{H}), 7.54-7.58(\mathrm{~m}, 2 \mathrm{H}), 7.61-7.67(\mathrm{~m}, 2 \mathrm{H})$; ${ }^{13} \mathrm{C}\{\mathrm{H}\}$ NMR $\left(125 \mathrm{MHz}, \mathrm{CDCl}_{3}\right) 21.0,32.3,44.8,46.8,60.5,71.4,87.2,110.1,122.5,123.1(\times 2)$, 123.8, 124.6, 127.7 ( $\times 2), 128.5,128.9,129.2,129.6,132.3,135.6,136.1,136.2,137.5,141.9,142.4$, 143.7, 174.2, 196.9, 200.50; HRMS (ES+) calcd for $\mathrm{C}_{34} \mathrm{H}_{26} \mathrm{~N}_{2} \mathrm{O}_{5} \mathrm{Na}(\mathrm{MNa}+)$ 565.1734, found 565.1736; Selected X-ray Data (CCDC 2034765): $\mathrm{C}_{34} \mathrm{H}_{26} \mathrm{~N}_{2} \mathrm{O}_{5}, M$ 542.57, Monoclinic, space group P21/n, $\mathrm{a}=$ 14.5193(10) $\AA, b=13.1808(12) \AA ̊, c=14.5248(13) \AA, \alpha=90^{\circ}, \beta=92.583(7)^{\circ}, \gamma=90^{\circ}, V=2776.9(4)$ $\AA^{3}, \rho=1.298 \mathrm{~g} / \mathrm{cm}^{3}, Z=4, F(000)=1136.0, \lambda=0.71073 \AA$, $\mu=0.088 \mathrm{~mm}^{-1}$, Total $/$ unique reflections $=$ $12144 / 4834[\mathrm{R}($ int $)=0.0836], \mathrm{T}=150 \mathrm{~K}, \theta$ range $=4.18$ to $50.00^{\circ}$, Final $\mathrm{R}[\mathrm{I}>2 \sigma(\mathrm{I})]: \mathrm{R}_{1}=0.0693$, $\mathrm{wR}_{2}=0.1352, \mathrm{R}$ (all data): $\mathrm{R}_{1}=0.1335, \mathrm{wR}_{2}=0.1744$.

\section{1'-Benzyl-5-nitro-2-phenyl-3a,5,6,6a-tetrahydro- $1 H$-spiro[cyclopenta[c]pyrrole-4,3'-indoline]-}

1,2',3(2H)-trione (7a). White solid; Yield $54 \mathrm{mg}, 58 \%$; Single isomer (compound isolated using silica

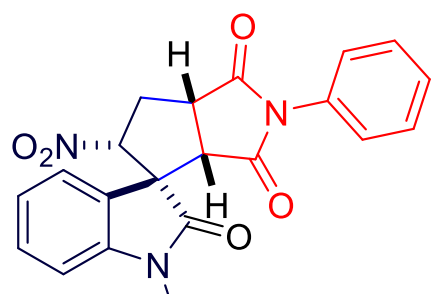

$\mathrm{Bn}$ gel column chromatography by eluting with $75 / 25$ petroleum ether/ethyl acetate); $\mathrm{mp} 227.5-229.5^{\circ} \mathrm{C}$; IR (KBr film) 3030 (vw), 2923 (vw), 1716 (vs), 1612 (w), 1552 (m), 1490 (w), 1468 (w), 1376 (s), 1181 (m), 757 (s) $\mathrm{cm}^{-1} ;{ }^{1} \mathrm{H}$ NMR $\left(400 \mathrm{MHz}, \mathrm{CDCl}_{3}\right) \delta 3.17-3.29(\mathrm{~m}, 2 \mathrm{H}), 3.85-3.95$ (unresolved $\mathrm{m}, 2 \mathrm{H}), 4.99-5.05(\mathrm{ABq}, J=15.9 \mathrm{~Hz}, 2 \mathrm{H}), 5.33(\mathrm{t}, J=7.4$ $\mathrm{Hz}, 1 \mathrm{H}), 6.78$ (d, $J=7.6 \mathrm{~Hz}, 1 \mathrm{H}), 6.99-7.01$ (unresolved $\mathrm{m}, 2 \mathrm{H}), 7.10$ $(\mathrm{d}, J=7.1 \mathrm{~Hz}, 2 \mathrm{H}), 7.26-7.43(\mathrm{~m}, 9 \mathrm{H}) ;{ }^{13} \mathrm{C}\{\mathrm{H}\} \mathrm{NMR}\left(125 \mathrm{MHz}, \mathrm{CDCl}_{3}\right)$ 31.3, 42.8, 44.7, 51.6, 59.0, 90.3, 110.6, 121.8, 123.3, 124.8, 126.5, 127.2, 128.1, 129.1, 129.2, 129.4, 130.9, 131.6, 134.8, 143.7, 172.5, 175.2, 176.8; HRMS (ESI) m/z: $[\mathrm{M}+\mathrm{H}]^{+}$Calcd for $\mathrm{C}_{27} \mathrm{H}_{22} \mathrm{~N}_{3} \mathrm{O}_{5}$ 468.1554; Found 468.1553.

1'-Benzyl-5-nitro-2-(p-tolyl)-3a,5,6,6a-tetrahydro-1H spiro[cyclopenta[c]pyrrole-4,3' indoline]1,2',3(2H)-trione (7b). White solid; Yield $46 \mathrm{mg}$, 48\%; Single diastereomer (compound isolated using silica gel column chromatography by eluting with $72 / 25$ petroleum ether/ethyl acetate); $\mathrm{mp} 186-188^{\circ} \mathrm{C}$;

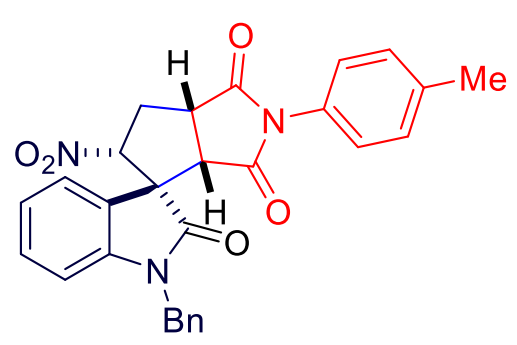
IR (KBr film) 3022 (vw), 2925 (vw), 1716 (vs), 1612 (v), 1553 (m), $1379(\mathrm{~s}), 1183(\mathrm{~m}), 1039(\mathrm{w}), 755(\mathrm{~s}) \mathrm{cm}^{-1} ;{ }^{1} \mathrm{H}$ NMR $(500 \mathrm{MHz}$, $\left.\mathrm{CDCl}_{3}\right) \delta 2.35(\mathrm{~s}, 3 \mathrm{H}), 3.14-3.19(\mathrm{~m}, 1 \mathrm{H}), 3.21-3.28(\mathrm{~m}, 1 \mathrm{H}), 3.83-$ $3.90(\mathrm{~m}, 2 \mathrm{H}), 5.00,5.04(\mathrm{ABq}, J=15.8 \mathrm{~Hz}, 2 \mathrm{H}), 5.33(\mathrm{t}, J=7.8 \mathrm{~Hz}$, $1 \mathrm{H}), 6.77(\mathrm{~d}, J=7.9 \mathrm{~Hz}, 1 \mathrm{H}), 6.97-7.00(\mathrm{~m}, 4 \mathrm{H}), 7.22(\mathrm{~d}, J=8.1$ $\mathrm{Hz}, 2 \mathrm{H}), 7.24-7.30(\mathrm{~m}, 2 \mathrm{H}), 7.34-7.35(\mathrm{~m}, 4 \mathrm{H})$; Confirmed by ${ }^{1} \mathrm{H}-$ ${ }^{1} \mathrm{H}$ COSY and ${ }^{1} \mathrm{H}-{ }^{1} \mathrm{H}$ NOESY experiments; ${ }^{13} \mathrm{C}\{\mathrm{H}\}$ NMR $(125$ $\left.\mathrm{MHz}, \mathrm{CDCl}_{3}\right)$ 21.4, 31.2, 42.7, 44.7, 51.6, 59.0, 90.3, 110.6, 121.9, 123.3, 124.8, 126.3, 127.2, 128.1, 128.9, 129.2, 130.1, 130.9, 134.8, 139.3, 143.8, 172.6, 175.2, 176.9; HRMS (ESI) m/z: $[\mathrm{M}+\mathrm{H}]^{+}$Calcd for $\mathrm{C}_{28} \mathrm{H}_{24} \mathrm{~N}_{3} \mathrm{O}_{5} 482.1710$; Found 482.1713.

\section{General procedure for gram scale synthesis of $3 a$}
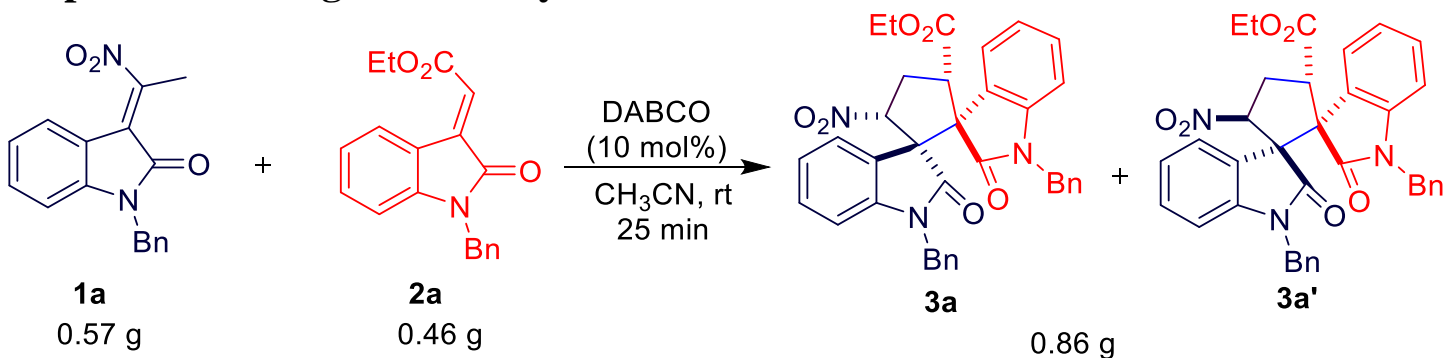

$0.86 \mathrm{~g}$

To a mixture of 3-(1-nitroalkylidene)-2-oxindole $1 \mathrm{a}(0.57 \mathrm{~g}, 1.95 \mathrm{mmol}, 1.3$ equiv) and 3alkylideneoxindole $2 \mathbf{a}(0.46 \mathrm{~g}, 1.5 \mathrm{mmol}, 1.0$ equiv) in acetonitrile $(8.0 \mathrm{~mL})$ was added DABCO (17.0 $\mathrm{mg}, 0.15 \mathrm{mmol}, 0.1$ equiv) at room temperature. The resulting reaction mixture was stirred at the same 
temperature for $25 \mathrm{~min}$. After completion of the reaction (monitored by TLC), the reaction mixture was concentrated in vacuo and the aqueous layer was extracted with ethyl acetate $(3 \times 30 \mathrm{~mL})$. The resulting combined organic layer was dried over $\mathrm{Na}_{2} \mathrm{SO}_{4}$ and concentrated in vacuo. Thereafter, the crude residue was purified by fractional crystallization from ethanol and water (90:10), which provided the desired pure 3a in $95 \%$ yield.

\section{General procedure for the synthesis of $\boldsymbol{\gamma}$-amino esters (9)}

To a solution of diastereomerically pure $3(0.1 \mathrm{mmol})$ in ethanol $(1 \mathrm{~mL})$ at room temperature, zinc $(130$ $\mathrm{mg}, 2.0 \mathrm{mmol})$ and acetic acid $(0.2 \mathrm{~mL})$ were added. After stirring at $50{ }^{\circ} \mathrm{C}$ (Preheated oil bath) for 6-8 $\mathrm{h}$, the reaction mixture was cooled to room temperature, and saturated aqueous $\mathrm{NH}_{4} \mathrm{Cl}$ was added. The aqueous phase was extracted with $\mathrm{CH}_{2} \mathrm{Cl}_{2}(4 \times 8 \mathrm{~mL})$, and the combined organic layer was washed with brine $(10 \mathrm{~mL})$, and dried over $\mathrm{Na}_{2} \mathrm{SO}_{4}$. After filtration, the solvent was removed and the residue was purified by column chromatography on neutral alumina by gradient elution with ethyl acetate/petroleum ether (4/6 to 9/1), to afford 9a and $9 \mathbf{b}$.

Ethyl 3'-amino-1,1"'-dibenzyl-2,2"'-dioxodispiro[indoline-3,1'-cyclopentane-2',3"'-indoline]-5'carboxylate (9a). White solid; Yield $54.0 \mathrm{mg}$, 94\%; Single diastereomer (compound isolated using neutral alumina column chromatography by eluting with $7 / 3$ ethyl acetate/petroleum ether); mp 149-

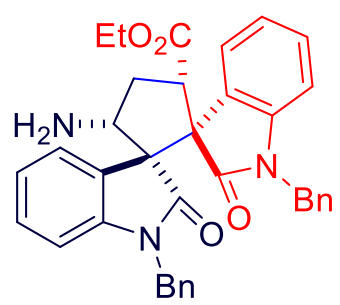

$151.5^{\circ} \mathrm{C}$; IR (KBr film) 3389 (br, w), 3027 (m), 2926 (m), 1734 (vs), 1711 (vs), 1609 (s), 1488 (s), 1467 (s), 1368 (vs), 1197 (s), 1180 (s), 755 (vs) cm $\mathrm{cm}^{-1}$; ${ }^{1} \mathrm{H}$ NMR $\left(400 \mathrm{MHz}, \mathrm{CDCl}_{3}\right) \delta 0.60(\mathrm{t}, J=7.1 \mathrm{~Hz}, 3 \mathrm{H}), 2.45$ (ddd collapsed to $\mathrm{dt}, J=12.1,7.6 \mathrm{~Hz}, 1 \mathrm{H}$ ), 3.54 (ddd collapsed to q, $J=12.1 \mathrm{~Hz}, 1 \mathrm{H}$ ), 3.62, 3.73 $(\mathrm{dq}, J=10.7,7.1 \mathrm{~Hz}, 2 \mathrm{H}), 3.89(\mathrm{dd}, J=12.1,7.6 \mathrm{~Hz}, 1 \mathrm{H}), 4.31(\mathrm{dd}, J=12.1$, $7.6 \mathrm{~Hz}, 1 \mathrm{H}), 4.39,5.05(\mathrm{~d}, J=16.0 \mathrm{~Hz}, 2 \mathrm{H}), 4.60,4.92(\mathrm{~d}, J=16.1 \mathrm{~Hz}, 2 \mathrm{H})$, 6.38 (overlapped d, $J=7.7 \mathrm{~Hz}, 1 \mathrm{H}$ ), 6.40 (overlapped d, $J=7.6 \mathrm{~Hz}, 1 \mathrm{H}$ ), 6.61 $(\mathrm{d}, J=7.4 \mathrm{~Hz}, 2 \mathrm{H}), 6.69(\mathrm{~d}, J=7.4 \mathrm{~Hz}, 2 \mathrm{H}), 6.80(\mathrm{t}, J=7.7 \mathrm{~Hz}, 1 \mathrm{H}), 6.89(\mathrm{t}, J=7.6 \mathrm{~Hz}, 1 \mathrm{H}), 6.99-7.14$ $(\mathrm{m}, 8 \mathrm{H}), 7.41(\mathrm{~d}, J=7.7 \mathrm{~Hz}, 1 \mathrm{H}), 7.54(\mathrm{~d}, J=7.6 \mathrm{~Hz}, 1 \mathrm{H}) ;{ }^{13} \mathrm{C}\{\mathrm{H}\}$ NMR $\left(100 \mathrm{MHz}, \mathrm{CDCl}_{3}\right)$ 13.6, 34.6, 43.3, 43.8, 49.2, 59.9, 60.3, 61.1, 65.9, $108.9(\times 2), 122.8,123.0,125.9,126.3,126.6,126.7,126.8$, 127.2, 127.3, 127.5, $128.7(\times 3), 129.0,135.0,135.3,143.2,144.3,170.8,174.9,178.7$; HRMS (ESI) $\mathrm{m} / \mathrm{z}:[\mathrm{M}+\mathrm{H}]^{+}$Calcd for $\mathrm{C}_{36} \mathrm{H}_{34} \mathrm{~N}_{3} \mathrm{O}_{4}$ 572.2544; Found 572.2543.

Ethyl 3'-amino-1,1"-dibenzyl-5,5"'-dimethyl-2,2"-dioxodispiro[indoline-3,1'-cyclopentane-2',3"-

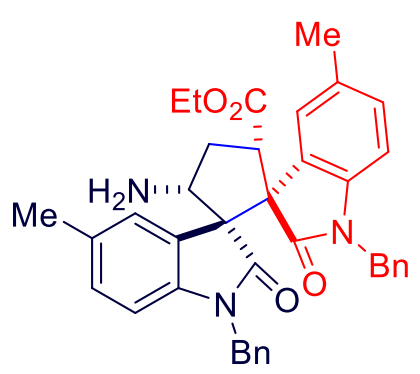
indoline]-5'-carboxylate (9b), Pale yellow solid; Yield $53.0 \mathrm{mg}, 88 \%$; Single diastereomer (compound isolated using neutral alumina column chromatography by eluting with $9 / 1$ ethyl acetate/petroleum ether); mp 154-156 ${ }^{\circ} \mathrm{C}$; IR (KBr film) 3391 (w), 2924 (m), 1738 (s), 1706 (vs), 1615 (m), $1602(\mathrm{~m}), 1497(\mathrm{~s}), 1366(\mathrm{~s}), 1195(\mathrm{~s}), 809(\mathrm{w}), 756(\mathrm{~s}) \mathrm{cm}^{-1} ;{ }^{1} \mathrm{H}$ NMR $\left(400 \mathrm{MHz} \mathrm{CDCl}_{3}\right) \delta 0.62(\mathrm{t}, J=7.1 \mathrm{~Hz}, 3 \mathrm{H}), 2.04(\mathrm{~s}, 3 \mathrm{H}), 2.11(\mathrm{~s}, 3 \mathrm{H})$, 2.44 (ddd collapsed to dt, $J=12.1,6.9 \mathrm{~Hz}, 1 \mathrm{H}$ ), 3.54 (ddd collapsed to q, $J=12.1 \mathrm{~Hz}, 1 \mathrm{H}), 3.64(\mathrm{dq}, J=10.7,7.1 \mathrm{~Hz}, 1 \mathrm{H}) 3.75(\mathrm{dq}, J=10.7,7.1$ $\mathrm{Hz}, 1 \mathrm{H}), 3.85(\mathrm{dd}, J=12.1,6.9 \mathrm{~Hz}, 1 \mathrm{H}), 4.32(\mathrm{dd}, J=12.1,6.9 \mathrm{~Hz}, 1 \mathrm{H}), 4.38(\mathrm{~d}, J=16.1 \mathrm{~Hz}, 1 \mathrm{H}), 4.59$ $(\mathrm{d}, J=16.2 \mathrm{~Hz}, 1 \mathrm{H}), 4.97(\mathrm{~d}, J=16.2 \mathrm{~Hz}, 1 \mathrm{H}), 5.09(\mathrm{~d}, J=16.1 \mathrm{~Hz}, 1 \mathrm{H}), 6.26(\mathrm{~d}, J=7.9 \mathrm{~Hz}, 1 \mathrm{H}), 6.28$ $(\mathrm{d}, J=7.9 \mathrm{~Hz}, 1 \mathrm{H}), 6.63(\mathrm{~d}, J=7.3 \mathrm{~Hz}, 2 \mathrm{H}), 6.71(\mathrm{~d}, J=7.3 \mathrm{~Hz}, 2 \mathrm{H}), 6.81(\mathrm{dd}, J=7.9,1.0 \mathrm{~Hz}, 1 \mathrm{H})$, $6.85(\mathrm{dd}, J=7.9,1.0 \mathrm{~Hz}, 1 \mathrm{H}), 7.01-7.15(\mathrm{~m}, 6 \mathrm{H}), 7.21(\mathrm{~d}, J=1.0 \mathrm{~Hz}, 1 \mathrm{H}), 7.35(\mathrm{~d}, J=1.0 \mathrm{~Hz}, 1 \mathrm{H})$; ${ }^{13} \mathrm{C}\{\mathrm{H}\} \mathrm{NMR}\left(100 \mathrm{MHz}, \mathrm{CDCl}_{3}\right)$ 13.6, 21.1, 21.3, 34.5, 43.2, 43.8, 49.1, 59.7, 60.3, 61.1, 66.1, 108.6, $108.7,126.2,126.4,126.5,126.6,127.1,127.2,127.4$ (× 2), 128.6, 128.7, 128.9, 129.3, 132.4, 132.5, 135.3, 135.5, 140.9, 141.9, 171.0, 174.9, 178.9; HRMS (ESI) m/z: $[\mathrm{M}+\mathrm{Na}]^{+}$Calcd for $\mathrm{C}_{38} \mathrm{H}_{38} \mathrm{~N}_{3} \mathrm{O}_{4}$ 600.2857; Found 600.2856. 
Sample preparation for crystal growth: Compound $\mathbf{1 g}$ was dissolved in ethanol, while slow evaporation of solvent at room temperature needle type red colour crystals were grown.

Table S1. Selected X-ray crystal data for $1 \mathrm{~g}$ with $50 \%$ probability for the ellipsoid contour (CCDC 2034250)

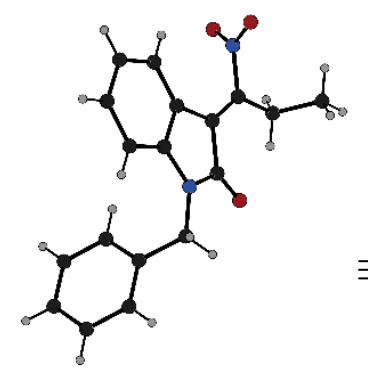

Identification code

Empirical formula

Formula weight

Temperature/K

Crystal system

Space group

$\mathrm{a} / \AA$

$\mathrm{b} / \AA$

$\mathrm{c} / \AA$

$\alpha /{ }^{\circ}$

$\beta /{ }^{\circ}$

$\gamma /{ }^{\circ}$

Volume/ $\AA^{3}$<smiles>C#CCC1C(=O)N(Cc2ccccc2)c2ccccc21</smiles>

$\mathrm{Z}$

$\rho_{\text {calc }} / \mathrm{cm}^{3}$

INN-CS-II-31_Mo

$\mathrm{C}_{18} \mathrm{H}_{16} \mathrm{~N}_{2} \mathrm{O}_{3}$

308.34

N/A

monoclinic

$\mathrm{P} 21 / \mathrm{n}$

11.4149(6)

$10.0055(6)$

13.8103(10)

90

106.044(7)

90

1515.86(17)

4

1.3510

0.093

$\mu / \mathrm{mm}^{-1}$

648.3

$\mathrm{F}(000)$

Crystal size $/ \mathrm{mm}^{3}$

$0.193 \times 0.093 \times 0.093$

Radiation

$2 \Theta$ range for data collection/ ${ }^{\circ}$

Mo K $\alpha(\lambda=0.71073)$

Index ranges

Reflections collected

4.12 to 50

$-15 \leq \mathrm{h} \leq 15,-10 \leq \mathrm{k} \leq 14,-19 \leq 1 \leq 19$

20498

Independent reflections

$2666\left[\mathrm{R}_{\text {int }}=0.1028, \mathrm{R}_{\text {sigma }}=0.0786\right]$

Data/restraints/parameters

Goodness-of-fit on $\mathrm{F}^{2}$

$2666 / 0 / 209$

1.041

Final R indexes $[\mathrm{I}>=2 \sigma(\mathrm{I})]$

$\mathrm{R}_{1}=0.0478, \mathrm{wR}_{2}=0.1115$

Final $\mathrm{R}$ indexes [all data]

$\mathrm{R}_{1}=0.0667, \mathrm{wR}_{2}=0.1266$

Largest diff. peak/hole / e $\AA^{-3}$

$0.40 /-0.51$ 
Sample preparation for crystal growth: Compound $\mathbf{3 m}$ was dissolved in hot ethanol, while slow evaporation of solvent at room temperature colourless square type crystals were grown.

Table S2. Selected X-ray data for 3m with $50 \%$ probability for the ellipsoid contour (CCDC 2035368)

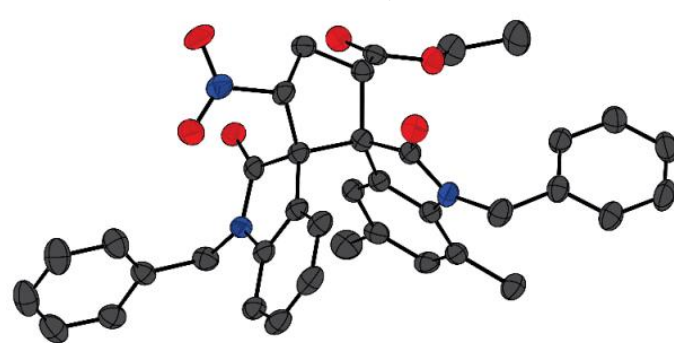

Identification code

Empirical formula

Formula weight

Temperature

Wavelength

Crystal system

Space group

Unit cell dimensions

Volume

Z

Density (calculated)

Absorption coefficient

$\mathrm{F}(000)$

Crystal size

Theta range for data collection

Index ranges

Reflections collected

Independent reflections

Completeness to theta $=25.00^{\circ}$

Absorption correction

Max. and min. transmission

Refinement method

Data / restraints / parameters

Goodness-of-fit on $\mathrm{F}^{2}$

Final $\mathrm{R}$ indices [I $>2 \operatorname{sigma}(\mathrm{I})]$

$\mathrm{R}$ indices (all data)

Largest diff. peak and hole<smiles>C#C[C@H]1C[C@]2(C(=O)N(Cc3ccccc3)c3c(C)cc(C)cc32)[C@@H](C(=O)OCC)C(=O)N1Cc1ccccc1</smiles>

inn-cs-01-188-a_mo

$\mathrm{C}_{38} \mathrm{H}_{35} \mathrm{~N}_{3} \mathrm{O}_{6}$

629.69

293(2) K

$0.71073 \AA$

Monoclinic

P 1 21/n 1

$\begin{array}{ll}\mathrm{a}=15.8155(9) \AA & \alpha=90^{\circ} . \\ \mathrm{b}=9.2877(6) \AA & \beta=100.843(6)^{\circ} . \\ \mathrm{c}=21.8270(15) \AA & \gamma=90^{\circ} .\end{array}$

3148.9(3) $\AA^{3}$

4

$1.328 \mathrm{Mg} / \mathrm{m}^{3}$

$0.091 \mathrm{~mm}^{-1}$

1328

$0.237 \times 0.126 \times 0.122 \mathrm{~mm}^{3}$

2.56 to $25.00^{\circ}$.

$-18<=\mathrm{h}<=18,-10<=\mathrm{k}<=11,-25<=\mathrm{l}<=22$

13984

$5515[\mathrm{R}(\mathrm{int})=0.0569]$

$99.7 \%$

Semi-empirical from equivalents

1.00000 and 0.96665

Full-matrix least-squares on $\mathrm{F}^{2}$

5515 / 0 / 427

1.071

$\mathrm{R} 1=0.0522, \mathrm{wR} 2=0.1089$

$\mathrm{R} 1=0.0838, \mathrm{wR} 2=0.1292$

0.252 and -0.281 e. $\AA^{-3}$ 
Sample preparation for crystal growth: Compound 3s' was dissolved in hot ethanol, while slow evaporation of solvent at room temperature square type colourless crystals were grown.

Table S3. Selected X-ray data for 3s' with 50\% probability for the ellipsoid contour (CCDC 2034424)

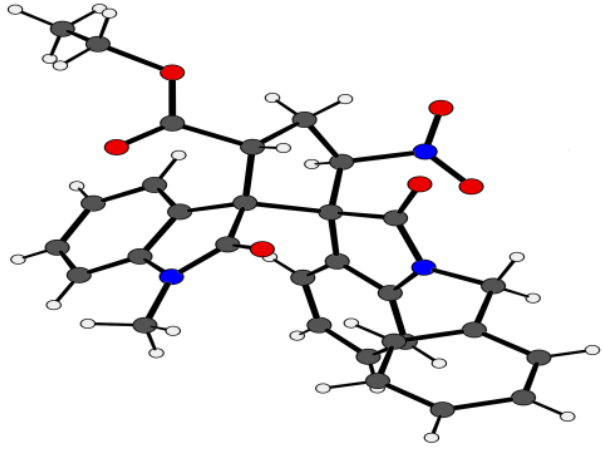

Identification code

Empirical formula

Formula weight

Temperature/K

Crystal system

Space group

$\mathrm{a} / \AA$

$\mathrm{b} / \AA$

$\mathrm{c} / \AA$

$\alpha /{ }^{\circ}$

$\beta /{ }^{\circ}$

$\gamma /{ }^{\circ}$

Volume $/ \AA^{3}$

$\mathrm{Z}$

$\rho_{\text {calc }} \mathrm{g} / \mathrm{cm}^{3}$

$\mu / \mathrm{mm}^{-1}$

$\mathrm{F}(000)$

Crystal size $/ \mathrm{mm}^{3}$

Radiation

$2 \Theta$ range for data collection/ ${ }^{\circ}$

Index ranges

Reflections collected

Independent reflections

Data/restraints/parameters

Goodness-of-fit on $\mathrm{F}^{2}$

Final $\mathrm{R}$ indexes $[\mathrm{I}>=2 \sigma(\mathrm{I})]$

Final $\mathrm{R}$ indexes [all data]

Largest diff. peak/hole / e $\AA^{-3}$

Flack parameter<smiles>C#CC[C@H]1C[C@@]2(C(=O)N(C)c3ccccc32)c2ccccc21</smiles>

INN-CS-01-164B-B_Mo

$\mathrm{C}_{30} \mathrm{H}_{27} \mathrm{~N}_{3} \mathrm{O}_{6}$

525.57

150

orthorhombic

$\mathrm{P} 2{ }_{1} 2{ }_{1}{ }_{1}$

9.8748(6)

$13.7568(8)$

19.0280(11)

90

90

90

2584.9(3)

4

1.3504

0.095

1104.6

$0.148 \times 0.085 \times 0.04$

$\operatorname{Mo~K\alpha }(\lambda=0.71073)$

4.64 to 50

$-10 \leq \mathrm{h} \leq 14,-18 \leq \mathrm{k} \leq 18,-26 \leq 1 \leq 26$

19191

$4536\left[\mathrm{R}_{\text {int }}=0.0742, \mathrm{R}_{\text {sigma }}=0.1151\right]$

$4536 / 0 / 353$

0.725

$\mathrm{R}_{1}=0.0511, \mathrm{wR}_{2}=0.1289$

$\mathrm{R}_{1}=0.0711, \mathrm{wR}_{2}=0.1561$

$0.27 /-0.31$

$-0.1(13)$ 
Sample preparation for crystal growth: Compound 4 was dissolved in hot ethanol, while slow evaporation of solvent at room temperature square type colourless crystals were grown.

Table S4. Selected X-ray data for 4 with 50\% probability for the ellipsoid contour (CCDC 2034765)

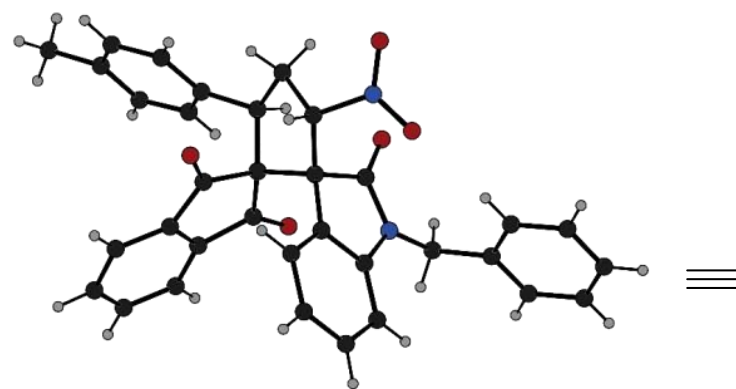

Identification code

Empirical formula

Formula weight

Temperature/K

Crystal system

Space group

$\mathrm{a} / \AA$

$\mathrm{b} / \AA$

$\mathrm{c} / \AA$

$\alpha /{ }^{\circ}$

$\beta /{ }^{\circ}$

$\gamma /{ }^{\circ}$

Volume $/ \AA^{3}$

Z

$\rho_{\text {calc }} \mathrm{g} / \mathrm{cm}^{3}$

$\mu / \mathrm{mm}^{-1}$

$\mathrm{F}(000)$

Crystal size $/ \mathrm{mm}^{3}$

Radiation

$2 \Theta$ range for data collection $/{ }^{\circ}$

Index ranges

Reflections collected

Independent reflections

Data/restraints/parameters

Goodness-of-fit on $\mathrm{F}^{2}$

Final $\mathrm{R}$ indexes $[\mathrm{I}>=2 \sigma(\mathrm{I})]$

Final $\mathrm{R}$ indexes [all data]

Largest diff. peak/hole / e $\AA^{-3}$<smiles>Cc1ccc([C@@H]2CC(N=O)[C@@]3(C(=O)c4ccccc4N(Cc4ccccc4)C3=O)C23C(=O)c2ccccc2C3=O)cc1</smiles>

INN-CS-01-177B_Mo

$\mathrm{C}_{34} \mathrm{H}_{26} \mathrm{~N}_{2} \mathrm{O}_{5}$

542.57

150

monoclinic

$\mathrm{P} 21 / \mathrm{n}$

14.5193(10)

$13.1808(12)$

$14.5248(13)$

90.00

92.583(7)

90.00

2776.9(4)

4

1.298

0.088

1136.0

$0.275 \times 0.074 \times 0.043$

$\operatorname{MoK} \alpha(\lambda=0.71073)$

4.18 to 50

$-12 \leq \mathrm{h} \leq 17,-12 \leq \mathrm{k} \leq 15,-17 \leq 1 \leq 15$

12144

$4834\left[\mathrm{R}_{\text {int }}=0.0836, \mathrm{R}_{\text {sigma }}=0.1315\right]$

$4834 / 0 / 371$

1.038

$\mathrm{R}_{1}=0.0693, \mathrm{wR}_{2}=0.1352$

$\mathrm{R}_{1}=0.1335, \mathrm{wR}_{2}=0.1744$

$0.33 /-0.33$ 


\section{Selected torsional angles}

Table S5. Selected torsional angles from X-ray data

\begin{tabular}{|c|c|c|}
\hline Chemical structure & Atom number & Torsional angle $\left({ }^{\circ}\right)$ \\
\hline 3m & $\begin{array}{c}\mathrm{C} 33-\mathrm{C} 2-\mathrm{C} 1-\mathrm{O} 1 \\
\text { N0-C33-C2-C1 } \\
\text { N0-C33-C2-C3 } \\
\text { O2-C17-C16-C35 } \\
\text { C17-C16-C35-C36 } \\
\text { C36-C35-C16-C18 } \\
\text { C1-C2-C16-C17 } \\
\text { C3-C2-C16-C18 } \\
\text { C17-C16-C2-C33 } \\
\text { C1-C2-C16-C35 } \\
\text { C35-C16-C2-C3 } \\
\text { C33-C2-C16-C18 } \\
\text { C17-C16-C2-C3 } \\
\text { C18-C16-C2-C1 }\end{array}$ & $\begin{array}{r}40.6 \\
47.9 \\
-69.9 \\
53.6 \\
120.0 \\
5.8 \\
154.7 \\
71.9 \\
88.1 \\
91.3 \\
-156.0 \\
-158.0 \\
-42.0 \\
-40.8\end{array}$ \\
\hline 3s' & $\begin{array}{c}\text { C21-C11-C12-C13 } \\
\text { O4-C13-C12-C11 } \\
\text { C21-C11-C12-C15 } \\
\text { N2-C9-C2-C1 } \\
\text { N2-C9-C2-C3 } \\
\text { C9-C2-C1-O1 } \\
\text { C1-C2-C12-C11 } \\
\text { C13-C12-C2-C1 } \\
\text { C1-C2-C12-C15 } \\
\text { C3-C2-C12-C13 } \\
\text { C9-C2-C12-C13 }\end{array}$ & $\begin{array}{l}76.0 \\
46.3 \\
-42.9 \\
50.0 \\
73.4 \\
-41.6 \\
-72.0 \\
51.1 \\
163.9 \\
-63.3 \\
166.7\end{array}$ \\
\hline
\end{tabular}




\begin{tabular}{|c|c|c|}
\hline & $\mathrm{C} 3-\mathrm{C} 2-\mathrm{C} 12-\mathrm{C} 15$ & 49.5 \\
& $\mathrm{C} 9-\mathrm{C} 2-\mathrm{C} 12-\mathrm{C} 15$ & -80.5 \\
& $\mathrm{C} 3-\mathrm{C} 2-\mathrm{C} 12-\mathrm{C} 11$ & -173.5 \\
\hline $\mathrm{Oe}$ & $\mathrm{O} 1-\mathrm{C}-\mathrm{C} 2-\mathrm{C} 16$ & -40.7 \\
\hline
\end{tabular}

\section{References}

(1) For preparation of 3-alkylideneoxindole esters 2: (a) 2a-d, 2i, and 2k: Liu, Y.; Xue, J.; Sun, Z.; Liu, D.; Xing, Y.; Li, Y. Substituent-Controlled Selective Synthesis of Spirooxindoles and Oxazolyloxindoles via Two Tandem Reactions. Asian J. Org. Chem. 2016, 5, 43. (b) 2h, 21 and 2o: Cao, S.-H.; Zhang, X.-C.; Wei, Y.; Shi, M. Chemoselective Reduction of Isatin-Derived Electron-Deficient Alkenes Using Alkylphosphanes as Reduction Reagents. Eur. J. Org. Chem. 2011, 2668. (c) 2e and 2g: Duan, S-.W.; Li, Y.; Liu, Y-.Y.; Zou, Y-.Q.; She, D-.Q.; Xiao, W-.J. An Organocatalytic Michael-aldol Cascade: Formal [3+2] Annulation to Construct Enantioenriched Spirocyclic Oxindole Derivatives. Chem. Commun. 2012, 48, 5160. (d) 2m: Satham, L.; Sankara, C. S.; Namboothiri, I. N. N. A MoritaBaylis-Hillman Pathway to Wittig Products: One-Pot Transformation of Nitroalkylideneoxindoles to Oxindolylidene Carboxylates. Eur. J. Org. Chem. 2020, 6903. (e) 2n: Cao, Y.; Jiang, X.; Liu, L.; Shen, F.; Zhang, F.; Wang, R. Enantioselective Michael/Cyclization Reaction Sequence: Scaffold-Inspired Synthesis of Spirooxindoles with Multiple Stereocenters. Angew. Chem. Int. Ed. 2011, 50, 9124. (f) 2f: Zhu, X.; Chiba, S. Construction of 1-Pyrroline Skeletons by Lewis Acid-Mediated Conjugate Addition of Vinyl Azides. Chem. Commun. 2016, 52, 2473. (g) 2j: Lin, W.-J.; Shia, K.-S.; Song, J.-S.; Wu, M. H.; Li, W.-T. Synthesis of (E)-Oxindolylideneacetate Using Tandem Palladium-Catalysed Heck and Alkoxycabonylation Reactions. Org. Biomol. Chem. 2016, 14, 220. For Wittig salts and reagents synthesis: (h) Osman, F. H.; El-Samahy, F. A. Phosphorus, Sulfur and Silicon 1998, 134/135, 437.

(2) Lou, Q.; Ding, Y.; Xu, D.; Liu, G.; Zhaob, J. Organocatalytic Enantioselective Synthesis of Dihydropyrano-indole Derivatives Bearing Trifluoromethylated All-Carbon- Substituted Stereocenters. Adv. Synth. Catal. 2017, 359, 2557.

(3) (a) Guo, S.; Zhang, H.; Huang, L.; Guo, Z.; Xiong, G.; Zhao, J. Porous Material-immobilized IodoBodipy as an Efficient Photocatalyst for Photoredox Catalytic Organic Reaction to Prepare Pyrrolo[2,1a]isoquinoline. Chem. Commun. 2013, 49, 8689. (b) Xu, W.-L.; Tang, L.; Ge, C.-Y.; Chen, J.; Zhoua, L. Synthesis of Tetrahydroisoindolinones via a Metal-Free Dehydrogenative Diels-Alder Reaction. Adv. Synth. Catal. 2019, 361, 2268.

(4) For experimental procedure for preparation of 1a-g, see: (a) Zhang, H.; Liu, Y.; Chen, R.; Xue, J.; Li, Y.; Tang, Y. Metal-Free Coupling of 3-Alkenyl Oxindoles by Nucleophilic Vinylic Substitution of 
Nitroolefins. Asian J. Org. Chem. 2013, 2, 307. (b) Ghosh, D.; Gupta, N.; Abdi, S. H. R.; Nandi, S.; Khan, N. H.; Kureshy, R. I.; Bajaj, H. C. Organocatalyzed Enantioselective Allylation of Isatins by Using a Chiral Amino Alcohol Derived Squaramide as Catalyst. Eur. J. Org. Chem. 2015, 2801. For experimental data of $\mathbf{1 h}$ and 11, see: (c) Otomasu, H.; Yoshida, K.; Natori, K. Unusual Reaction of 3Chloro-3-nitroalkylindolin-2-ones. Chem. Pharm. Bull. 1975, 23, 1436. And ref. 1d 


\begin{tabular}{|c|c|c|}
\hline Entry & Table of contents & Page \\
\hline 2 & Figure S2. ${ }^{13} \mathrm{C}$ NMR Spectrum of $\mathbf{1 1}$ & S31 \\
\hline 3 & Figure S3. ${ }^{13} \mathrm{C}$-APT NMR Spectrum of $\mathbf{1 1}$ & S32 \\
\hline 4 & Figure S4. ${ }^{1} \mathrm{H}$ NMR Spectrum of $\mathbf{1 a}$ & S33 \\
\hline 6 & Figure S6. ${ }^{1} \mathrm{H}$ NMR Spectrum of $\mathbf{1 b}$ & S35 \\
\hline 7 & Figure S7. ${ }^{13}$ C NMR Spectrum of $\mathbf{1 b}$ & S36 \\
\hline 8 & Figure S8. ${ }^{13}$ C-APT NMR Spectrum of $\mathbf{1 b}$ & S37 \\
\hline 9 & Figure S9. ${ }^{1} \mathrm{H}$ NMR Spectrum of 1c & S38 \\
\hline 12 & Figure S12. ${ }^{13} \mathrm{C}$ NMR Spectrum of $\mathbf{1 d}$ & S41 \\
\hline 13 & Figure S13. ${ }^{1} \mathrm{H}$ NMR Spectrum of $1 \mathrm{e}$ & S42 \\
\hline 14 & Figure S14. ${ }^{1} \mathrm{H}$ NMR Spectrum of $1 \mathrm{e}$ & S43 \\
\hline 15 & Figure S15. ${ }^{1} \mathrm{H}$ NMR Spectrum of $\mathbf{1 f}$ & S44 \\
\hline 16 & Figure S16. ${ }^{19}$ F NMR Spectrum of $\mathbf{1 f}$ & S45 \\
\hline
\end{tabular}




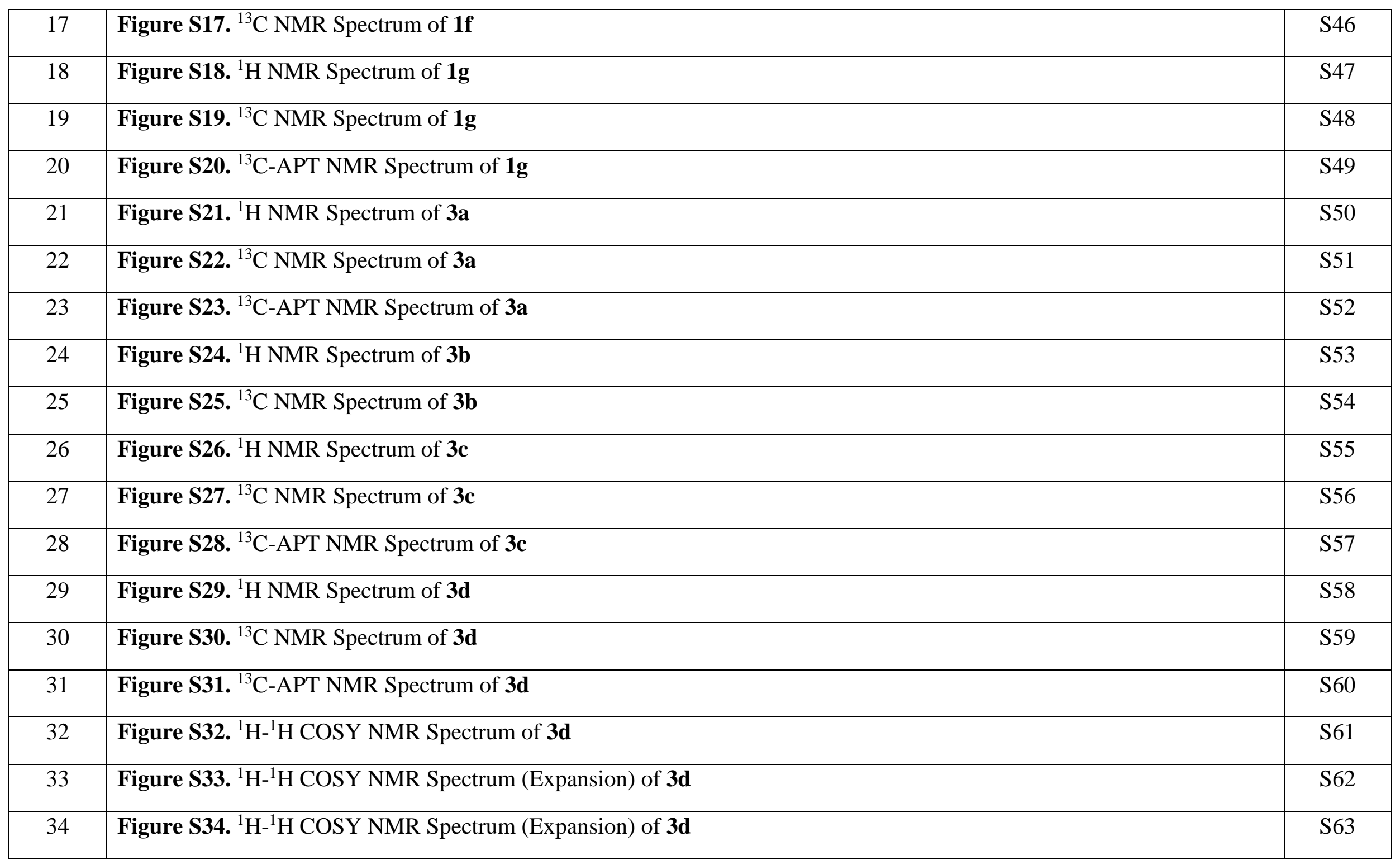




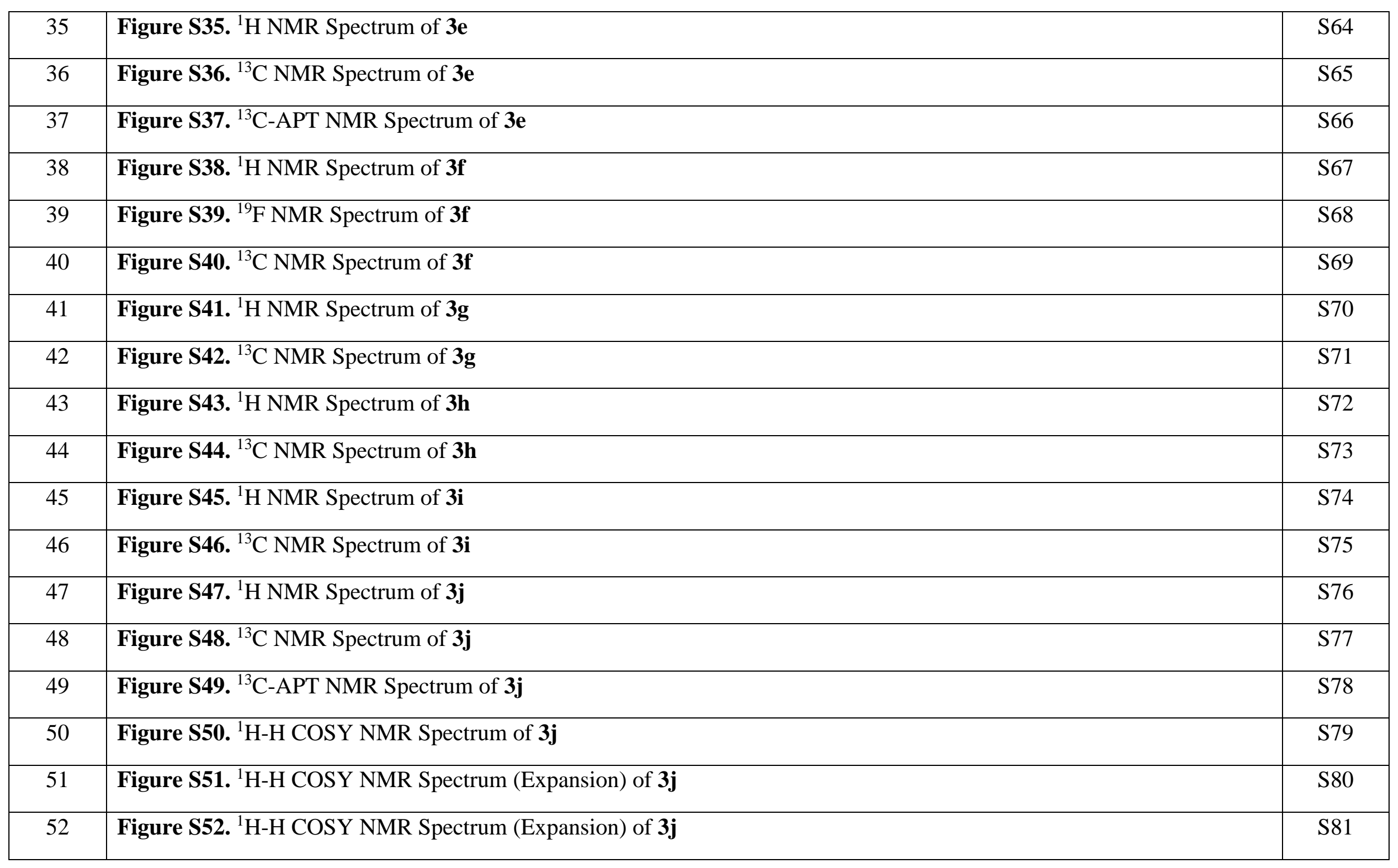




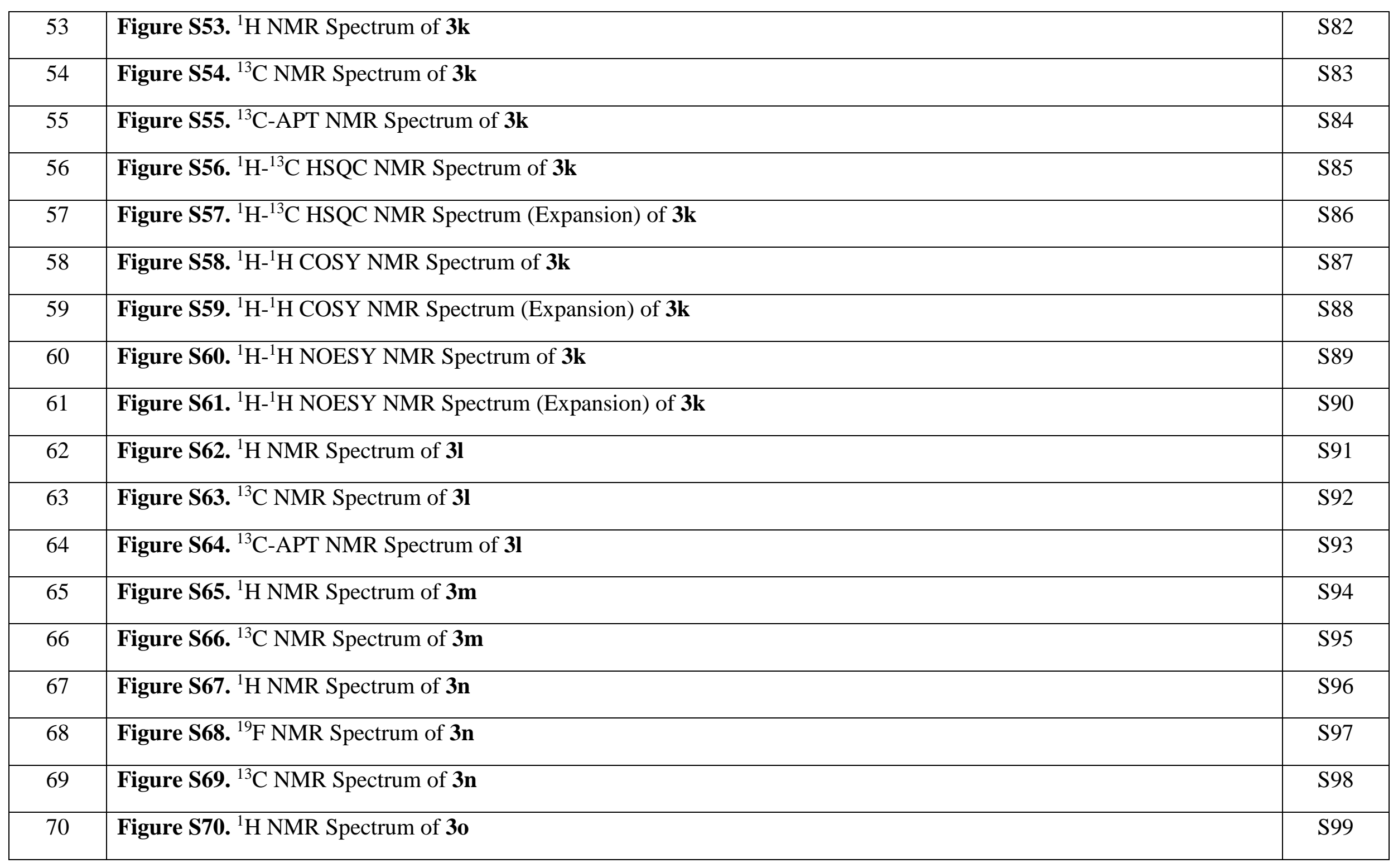




\begin{tabular}{|c|c|c|}
\hline 71 & Figure S71. ${ }^{13} \mathrm{C}$ NMR Spectrum of $\mathbf{3 o}$ & S100 \\
\hline 72 & Figure S72. ${ }^{13}$ C-APT NMR Spectrum of $\mathbf{3 o}$ & S101 \\
\hline 73 & Figure S73. ${ }^{1} \mathrm{H}$ NMR Spectrum of $\mathbf{3 p}$ & S102 \\
\hline 74 & Figure S74. ${ }^{13} \mathrm{C}$ NMR Spectrum of $\mathbf{3 p}$ & S103 \\
\hline 75 & Figure S75. ${ }^{13} \mathrm{C}$-APT NMR Spectrum of $\mathbf{3 p}$ & S104 \\
\hline 77 & Figure S77. ${ }^{1} \mathrm{H}-{ }^{1} \mathrm{H}$ COSY NMR Spectrum of $\mathbf{3 p}$ & S106 \\
\hline 78 & Figure S78. ${ }^{1} \mathrm{H}-{ }^{1} \mathrm{H}$ COSY NMR Spectrum (Expansion) of $\mathbf{3 p}$ & S107 \\
\hline 79 & Figure S79. ${ }^{1} \mathrm{H}-{ }^{1} \mathrm{H}$ NOESY NMR Spectrum of $\mathbf{3 p}$ & S108 \\
\hline 80 & Figure S80. ${ }^{1} \mathrm{H}-{ }^{1} \mathrm{H}$ NOESY NMR Spectrum (Expansion) of 3p & S109 \\
\hline 83 & Figure S83. ${ }^{1} \mathrm{H}$ NMR Spectrum of $\mathbf{3 q}$ & S112 \\
\hline 84 & Figure S84. ${ }^{13} \mathrm{C}$ NMR Spectrum of $\mathbf{3 q}$ & S113 \\
\hline 85 & Figure S85. ${ }^{1} \mathrm{H}$ NMR Spectrum of $\mathbf{3 r}$ & S114 \\
\hline 86 & Figure S86. ${ }^{13} \mathrm{C}$ NMR Spectrum of $\mathbf{3 r}$ & S115 \\
\hline 87 & Figure S87. ${ }^{1} \mathrm{H}$ NMR Spectrum of $\mathbf{3 s}$ & S116 \\
\hline 88 & Figure S88. ${ }^{13} \mathrm{C}$ NMR Spectrum of $\mathbf{3 s}$ & S117 \\
\hline
\end{tabular}




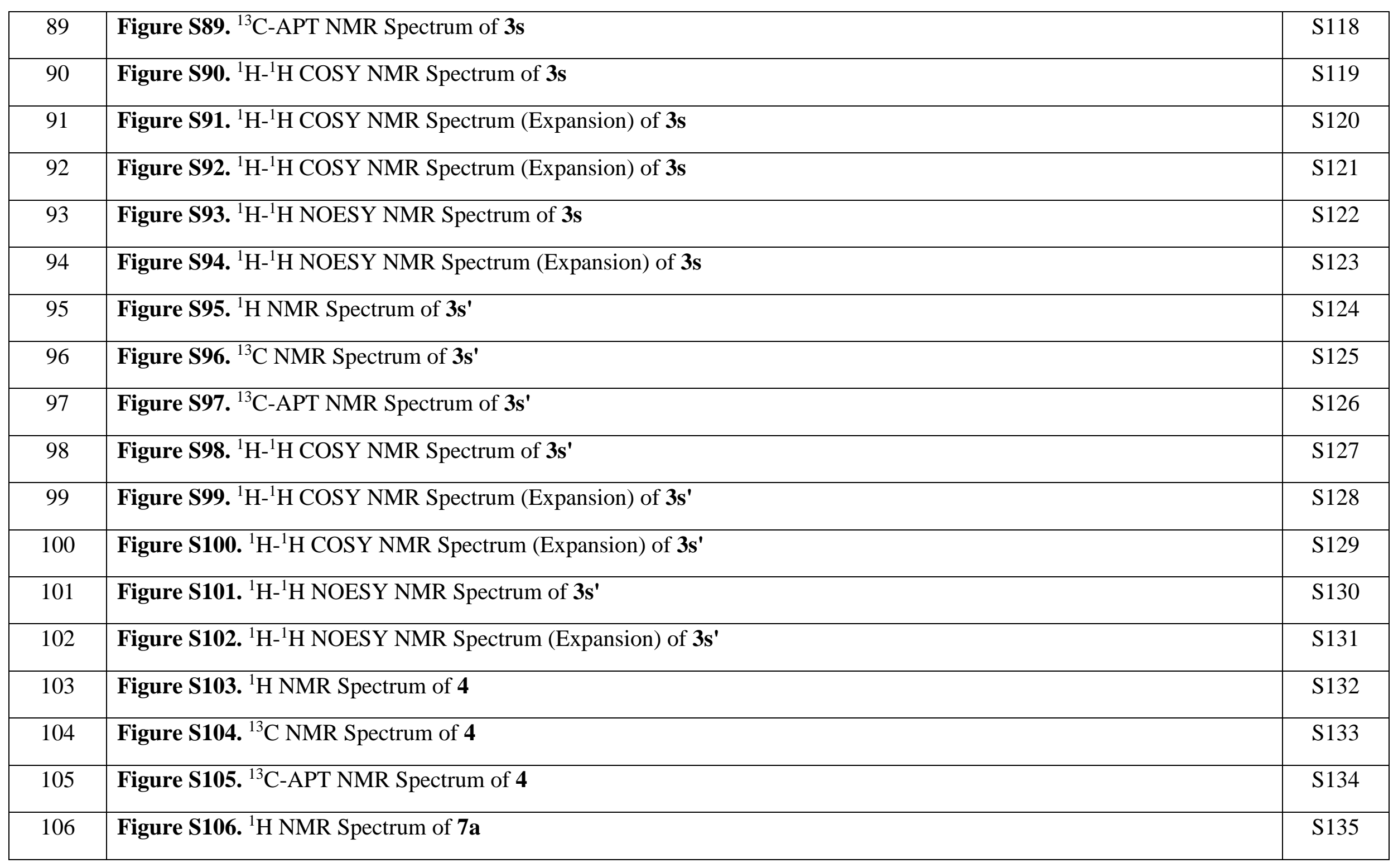




\begin{tabular}{|c|c|c|}
\hline 107 & Figure S107. ${ }^{13} \mathrm{C}$ NMR Spectrum of $\mathbf{7 a}$ & S136 \\
\hline 108 & Figure S108. ${ }^{13} \mathrm{C}$-APT NMR Spectrum of 7a & S137 \\
\hline 109 & Figure S109. ${ }^{1} \mathrm{H}$ NMR Spectrum of $\mathbf{7 b}$ & S138 \\
\hline 110 & Figure S110. ${ }^{13} \mathrm{C}$ NMR Spectrum of $\mathbf{7 b}$ & S139 \\
\hline 111 & Figure S111. ${ }^{13} \mathrm{C}$-APT NMR Spectrum of $\mathbf{7 b}$ & S140 \\
\hline 113 & Figure S113. ${ }^{1} \mathrm{H}-{ }^{1} \mathrm{H}$ COSY NMR Spectrum (Expansion) of $\mathbf{7 b}$ & S142 \\
\hline 114 & Figure S114. ${ }^{1} \mathrm{H}-{ }^{1} \mathrm{H}$ NOESY NMR Spectrum of $\mathbf{7 b}$ & S143 \\
\hline 115 & Figure S115. ${ }^{1} \mathrm{H}-{ }^{1} \mathrm{H}$ NOESY NMR Spectrum (Expansion) of $\mathbf{7 b}$ & S144 \\
\hline 117 & Figure S116. ${ }^{1} \mathrm{H}$ NMR Spectrum of $9 a$ & S145 \\
\hline 120 & Figure S119. ${ }^{1} \mathrm{H}$ NMR Spectrum of $\mathbf{9 b}$ & S148 \\
\hline 121 & Figure $\mathbf{S 1 2 0} .{ }^{13} \mathrm{C}$ NMR Spectrum of $\mathbf{9 b}$ & S149 \\
\hline 122 & Figure S121. ${ }^{13} \mathrm{C}$-APT NMR Spectrum of $9 \mathrm{~b}$ & S150 \\
\hline 123 & Figure S122. DEPT NMR Spectrum of $9 \mathbf{b}$ & S151 \\
\hline
\end{tabular}


Table S6. ${ }^{13} \mathrm{C}$ NMR $\left(\delta, \mathrm{CDCl}_{3}\right.$, major) data of 3

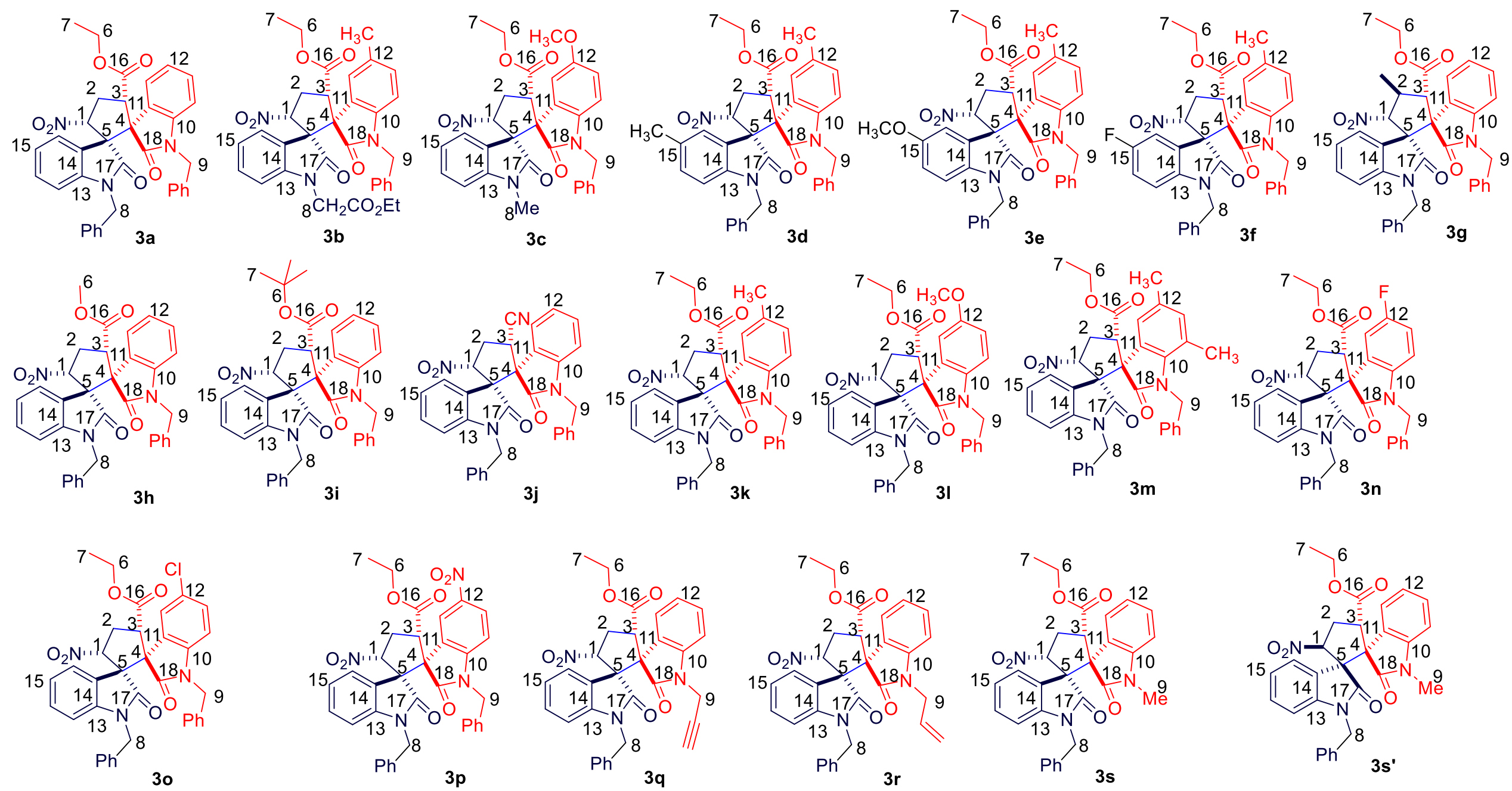




\begin{tabular}{|c|c|c|c|c|c|c|c|c|c|c|c|c|c|c|c|c|c|c|}
\hline & 1 & 2 & 3 & 4 & 5 & 6 & 7 & 8 & 9 & 10 & 11 & 12 & 13 & 14 & 15 & 16 & 17 & 18 \\
\hline $3 \mathbf{a}$ & 86.7 & 30.1 & 47.7 & 60.5 & 60.9 & 60.9 & 13.6 & 43.9 & 43.9 & 144.0 & 124.2 & 123.1 & 143.3 & 124.9 & 123.0 & 169.3 & 178.1 & 173.8 \\
\hline $\mathbf{3 b}$ & 86.4 & 30.6 & 47.6 & 60.4 & 60.3 & 60.9 & 13.6 & 41.5 & 43.9 & 140.7 & 125.0 & 132.6 & 143.5 & 123.7 & 123.2 & 169.3 & 178.1 & 173.5 \\
\hline $3 c$ & 86.3 & 30.7 & 47.6 & 60.4 & 60.6 & 60.9 & 13.6 & 43.9 & 26.1 & 136.5 & 123.9 & 155.9 & 144.5 & 125.7 & 123.0 & 169.2 & 178.0 & 173.6 \\
\hline 3d & 86.7 & 30.9 & 47.6 & 60.5 & 60.5 & 60.9 & 13.6 & 43.8 & 43.8 & 141.6 & 124.0 & 132.5 & \begin{tabular}{|l}
140.9 \\
\end{tabular} & 124.8 & \begin{tabular}{|l|}
132.7 \\
\end{tabular} & 169.4 & 178.2 & 173.2 \\
\hline $3 e$ & 86.8 & 30.9 & 47.7 & 60.5 & 60.7 & 60.9 & 13.6 & 43.8 & 43.8 & 141.0 & 125.3 & 132.9 & 134.7 & 124.9 & 156.0 & 169.3 & 178.2 & 173.6 \\
\hline 3f & 86.8 & 30.8 & 47.7 & 60.5 & 60.6 & 61.0 & 13.6 & 44.0 & 44.0 & 141.0 & 125.9 & 133.0 & \begin{tabular}{|l|}
140.1 \\
\end{tabular} & 124.7 & 159.2 & 169.2 & 177.9 & 173.6 \\
\hline $3 g$ & 92.2 & 37.8 & 56.1 & 58.8 & 61.6 & 60.9 & 13.6 & 43.9 & 43.9 & 143.9 & 129.8 & 123.1 & 143.4 & 125.0 & 123.1 & 169.0 & 178.3 & 173.4 \\
\hline $3 h$ & 86.7 & 31.0 & 47.7 & 60.5 & 60.5 & 51.8 & - & 43.9 & 44.0 & 144.0 & 124.1 & 123.1 & 143.3 & 124.7 & 123.1 & 169.8 & 178.1 & 173.8 \\
\hline $3 \mathbf{i}$ & 86.7 & 31.4 & 48.4 & 60.7 & 60.8 & 81.7 & 27.8 & 43.9 & 43.9 & 144.0 & 124.6 & 123.2 & 143.4 & 129.6 & \begin{tabular}{|l|}
122.9 \\
\end{tabular} & 168.0 & 178.0 & 173.9 \\
\hline 3j & 85.5 & 32.3 & 32.8 & 58.9 & 60.4 & - & - & 44.3 & 44.4 & 144.0 & 130.6 & 122.6 & 143.1 & 130.1 & 121.6 & - & 176.9 & 173.8 \\
\hline $3 \mathbf{k}$ & 86.7 & 31.0 & 47.6 & 60.5 & 60.5 & 60.9 & 13.6 & 43.8 & 43.9 & 140.9 & 124.2 & 132.8 & 144.2 & 124.9 & 123.0 & 169.4 & 178.1 & 173.9 \\
\hline 31 & 86.8 & 31.0 & 47.8 & 60.6 & 60.7 & 61.0 & 13.7 & 43.9 & 44.0 & 136.6 & 124.2 & 156.1 & 144.2 & 126.0 & 123.1 & 169.3 & 177.9 & 173.8 \\
\hline $3 \mathrm{~m}$ & 86.7 & 30.9 & 48.1 & 59.9 & 60.7 & 60.9 & 13.7 & 43.8 & 45.2 & 138.9 & 124.3 & 134.1 & 144.2 & 125.3 & 123.0 & 169.4 & 179.1 & 173.9 \\
\hline $3 n$ & 86.6 & 30.9 & 47.7 & 60.5 & 60.5 & 61.2 & 13.7 & 44.0 & 44.1 & 139.3 & 126.5 & 159.1 & \begin{tabular}{|l|}
144.1 \\
\end{tabular} & 129.9 & 123.2 & 169.0 & 177.9 & 173.7 \\
\hline 30 & 86.6 & 30.9 & 47.7 & 60.4 & 60.5 & 61.3 & 13.6 & 44.1 & 44.1 & 141.9 & 123.7 & 128.7 & \begin{tabular}{|l|}
144.1 \\
\end{tabular} & 126.6 & 123.2 & 169.0 & 177.8 & 173.7 \\
\hline $3 p$ & 86.1 & 29.5 & 47.0 & 61.1 & 61.2 & 61.9 & 14.1 & 44.4 & 44.6 & 143.6 & 126.0 & 144.7 & 149.5 & 123.3 & \begin{tabular}{|l}
120.0 \\
\end{tabular} & 170.7 & 177.4 & 174.3 \\
\hline $3 q$ & 86.4 & 30.8 & 46.8 & 60.5 & 60.6 & 61.0 & 13.7 & 44.0 & 29.6 & 143.8 & 123.5 & 123.3 & \begin{tabular}{|l|l}
142.0 \\
\end{tabular} & 126.7 & \begin{tabular}{|l}
123.0 \\
\end{tabular} & 169.3 & 177.3 & 173.7 \\
\hline $3 r$ & 86.5 & 30.9 & 47.2 & 60.4 & 60.5 & 60.9 & 13.7 & 43.9 & 42.4 & 144.0 & 123.0 & 122.8 & 143.3 & 129.5 & 124.8 & 169.4 & 177.8 & 173.8 \\
\hline $3 s$ & 86.3 & 30.8 & 46.5 & 60.5 & 60.6 & 61.0 & 13.7 & 43.9 & 26.4 & 143.9 & 124.1 & 122.9 & 143.8 & 126.4 & 122.5 & 169.5 & 178.0 & 173.7 \\
\hline $3 s^{\prime}$ & 86.8 & 28.8 & 26.3 & 62.3 & 62.0 & 60.7 & 13.5 & 44.5 & 44.9 & 144.5 & 126.6 & 121.6 & \begin{tabular}{|l|l}
143.8 \\
\end{tabular} & 135.5 & \begin{tabular}{|l|}
122.5 \\
\end{tabular} & 170.2 & 173.4 & 172.3 \\
\hline
\end{tabular}

${ }^{a}$ The chemical shifts in ppm. The merged carbons (carbon numbers-4, 5 and 8,9) were shown with same ppm values. 


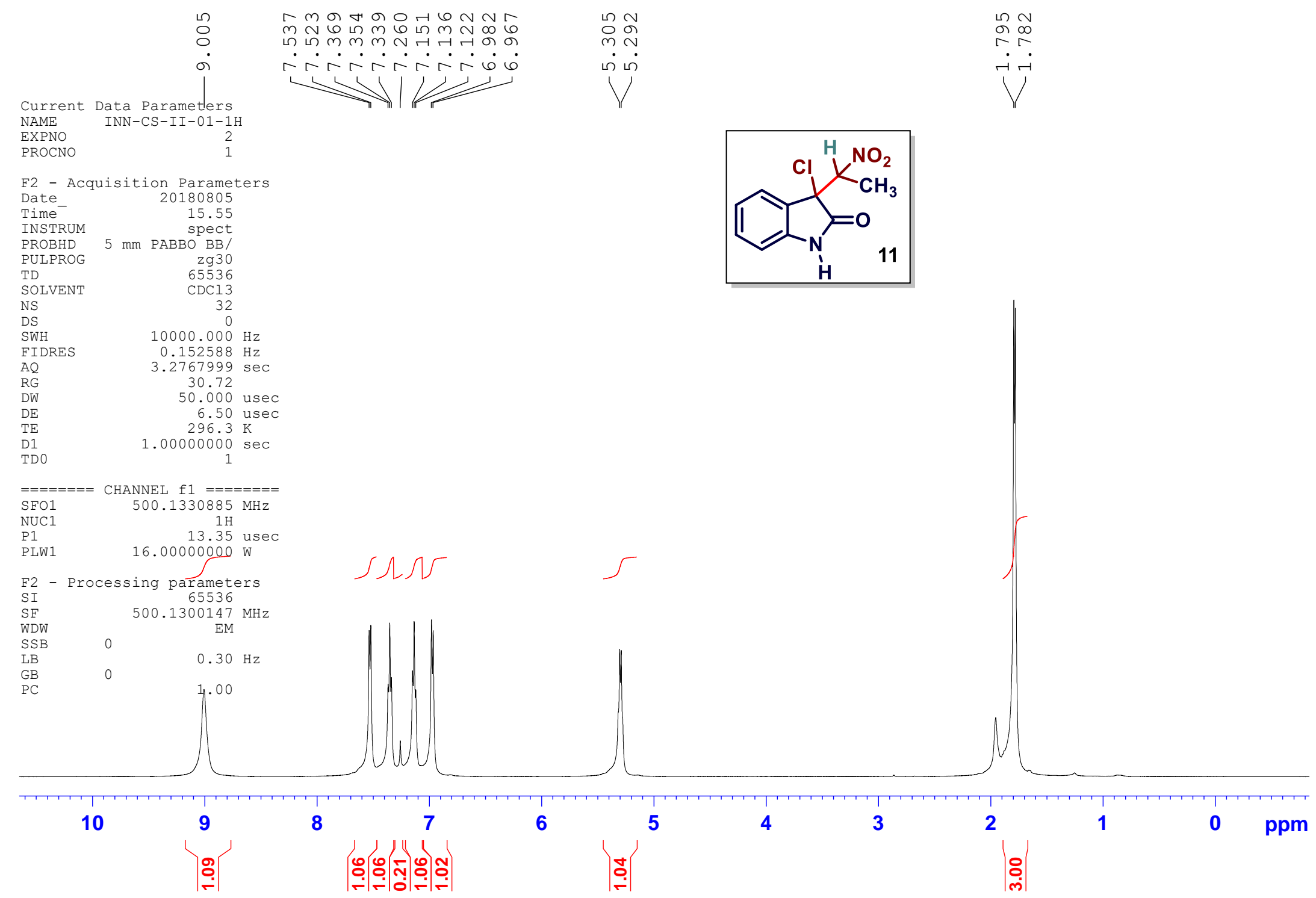

Figure S1. ${ }^{1} \mathrm{H}$ NMR $\left(500 \mathrm{MHz}, \mathrm{CDCl}_{3}\right)$ Spectrum of $\mathbf{1 1}$ 

$\stackrel{m}{\stackrel{m}{\infty}}$
|

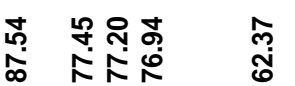

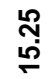
i

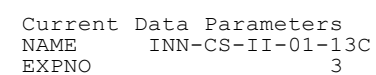

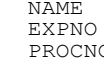

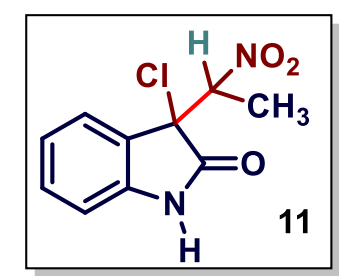

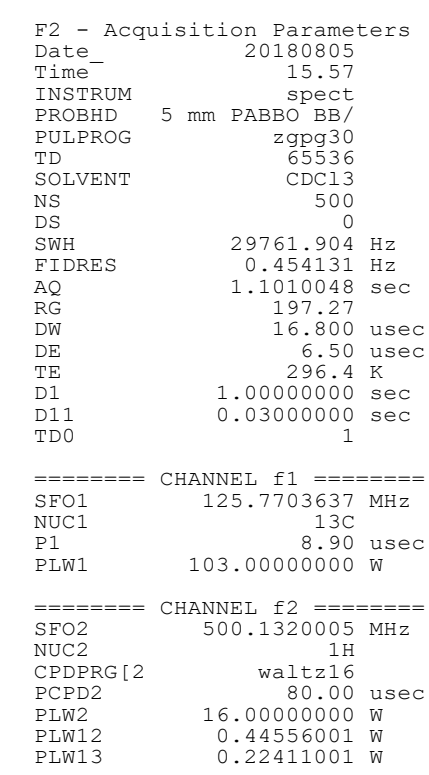

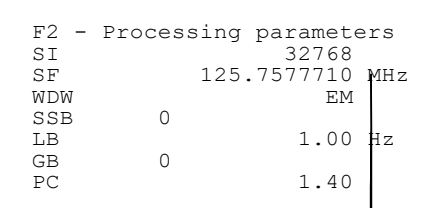

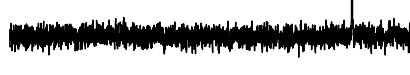

$\begin{array}{lllll}200 & 190 & 180 & 170 & 160\end{array}$

Figure S2. ${ }^{13} \mathrm{C}$ NMR $\left(125 \mathrm{MHz}, \mathrm{CDCl}_{3}\right)$ Spectrum of $\mathbf{1 1}$ 


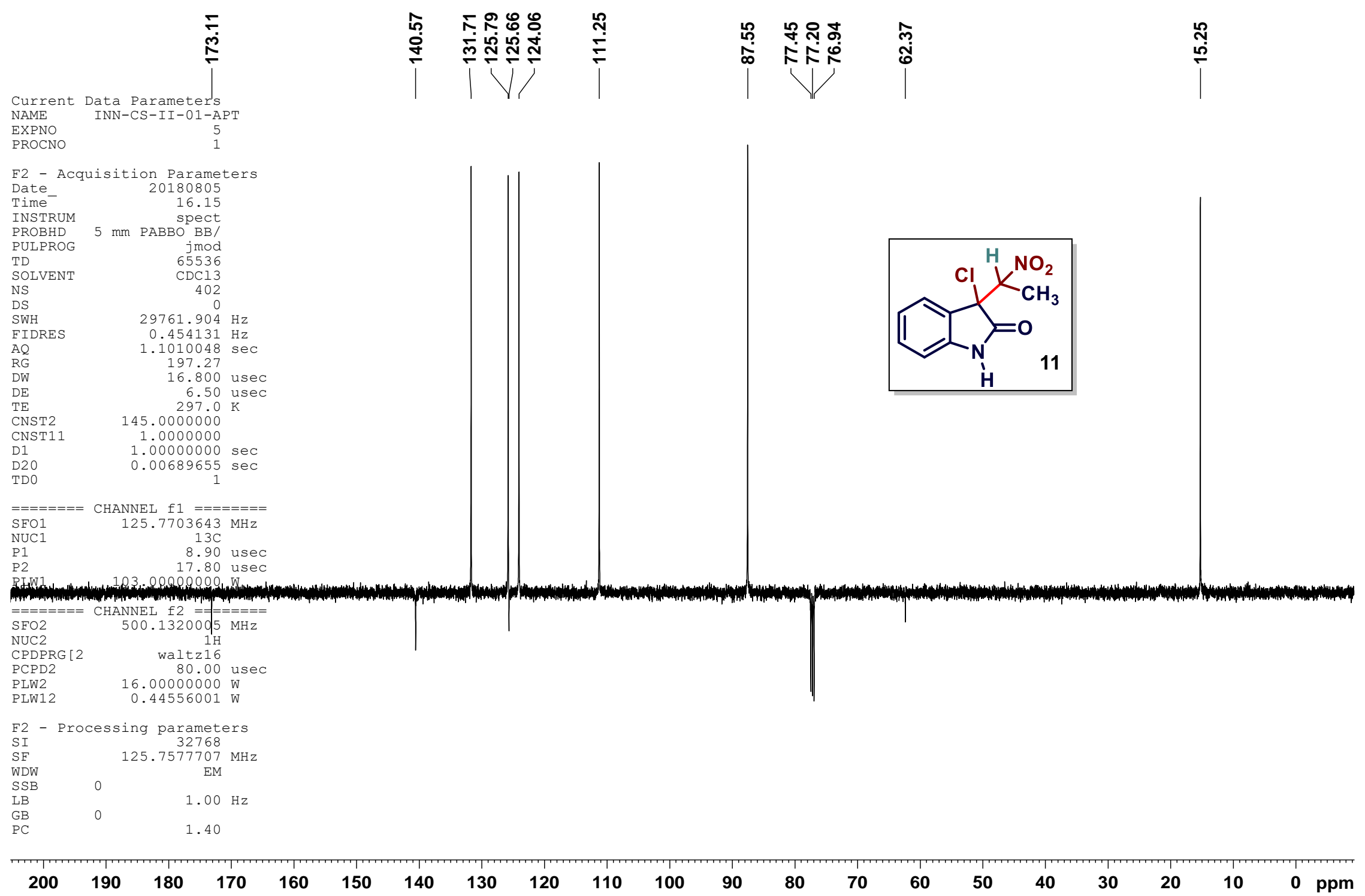

Figure S3. ${ }^{13} \mathrm{C}$-APT NMR (125 MHz, $\mathrm{CDCl}_{3}$ ) Spectrum of $\mathbf{1 1}$ 


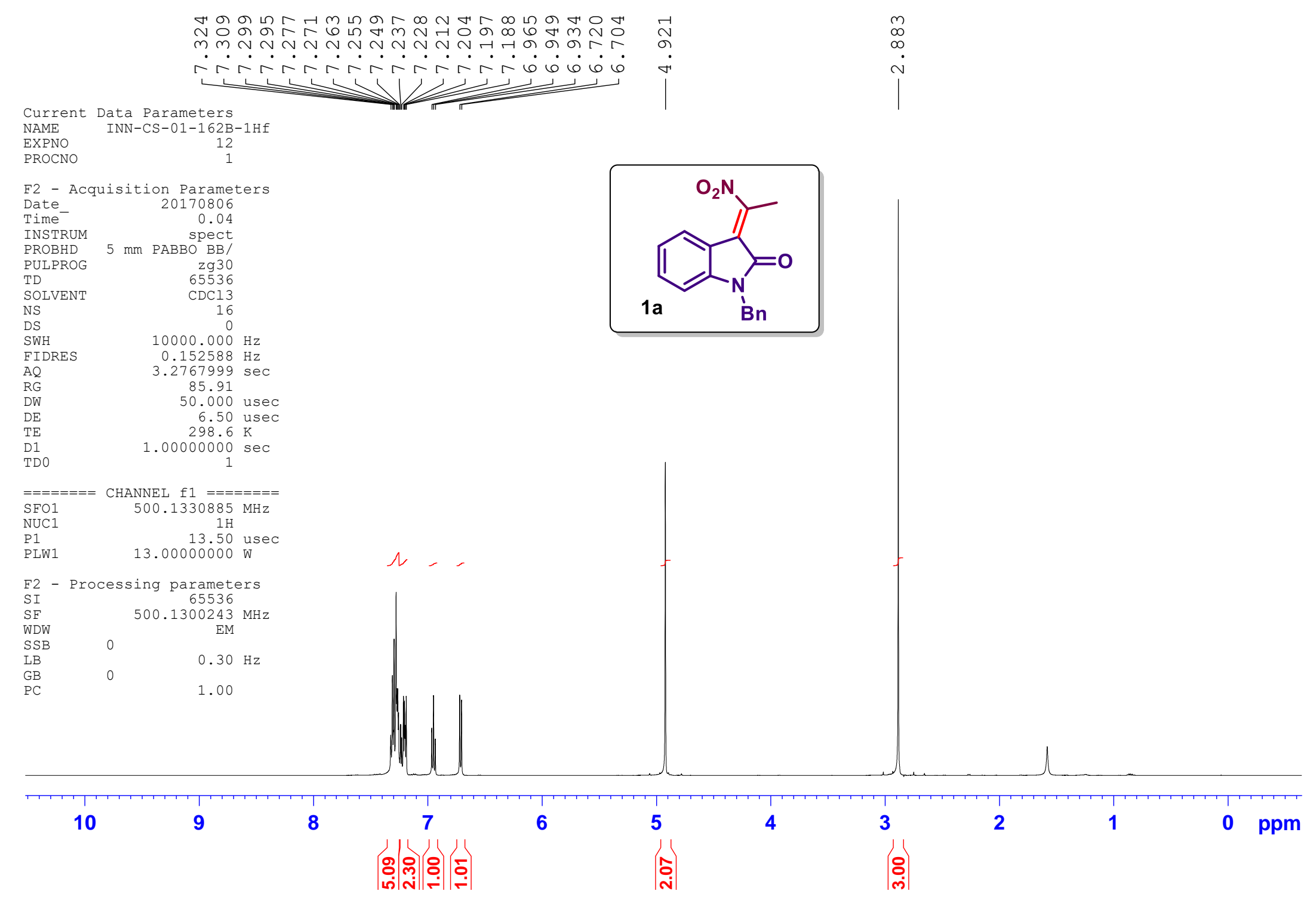

Figure S4. ${ }^{1} \mathrm{H}$ NMR $\left(500 \mathrm{MHz}, \mathrm{CDCl}_{3}\right)$ Spectrum of $\mathbf{1 a}$ 


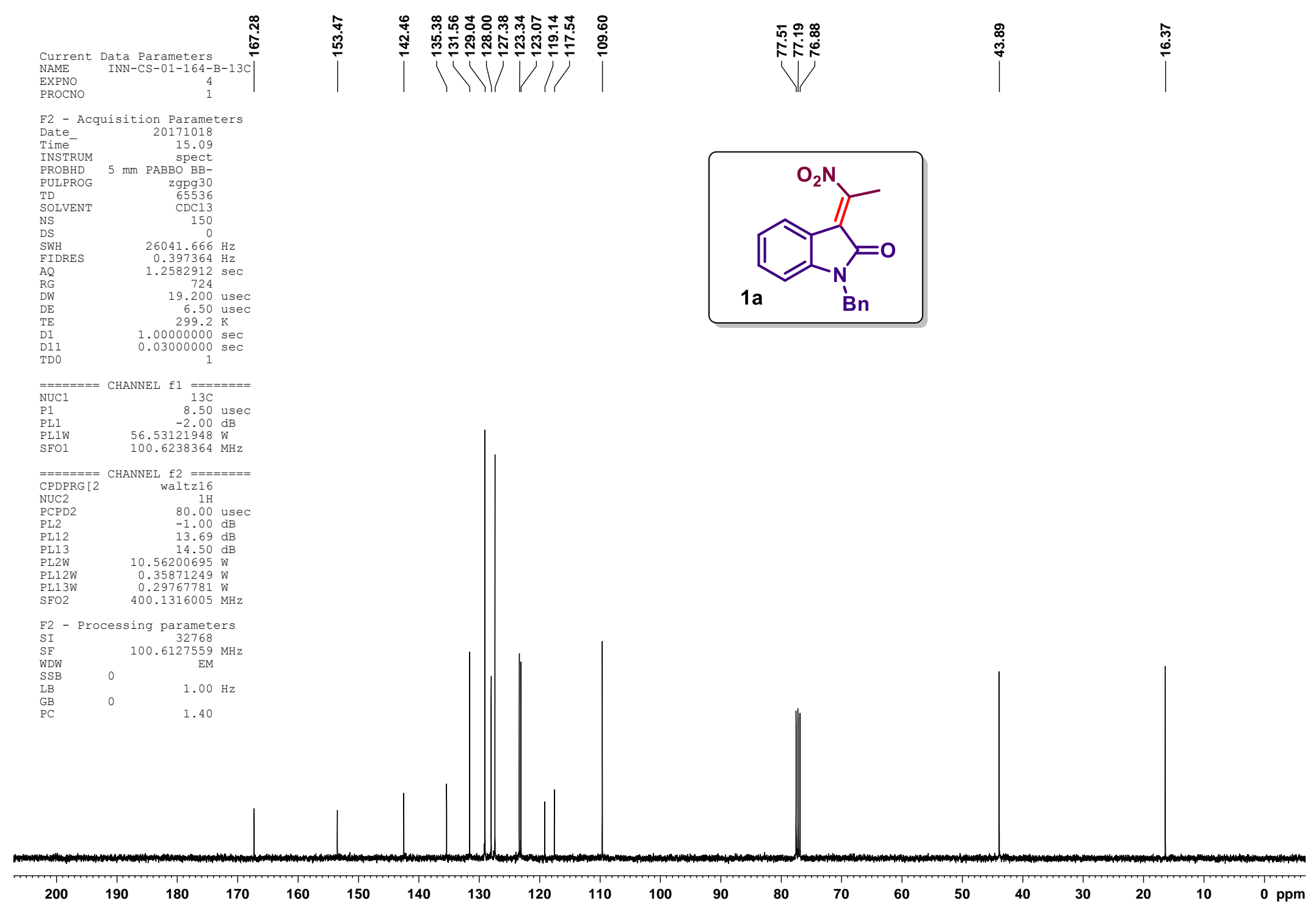

Figure S5. ${ }^{13} \mathrm{C}$ NMR $\left(100 \mathrm{MHz}, \mathrm{CDCl}_{3}\right)$ Spectrum of 1a 
Current Data Parameters
NAME
INN-CS-II-58-1H

NAME

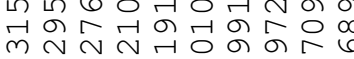

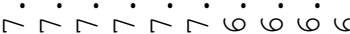

$\rightarrow 1$

F2 - Acquisition Parameters

20180510
11.13

Time

PROBHD

PULPROG

spect

Bg-

SOLVENT

zg30
54274

DS

$\begin{array}{ll}\text { FIDRES } & 0.151522 \mathrm{~Hz} \\ \mathrm{AQ} & 3.2998593 \mathrm{sec}\end{array}$

RG

$\begin{array}{lr}\text { DW } & 60.800 \text { usec } \\ \text { DE } & 6.50 \text { usec }\end{array}$

TE
D1
TD 0

$.00000000 \mathrm{sec}$

$=======$ CHANNEL $\mathrm{f} 1=======$
NUC1

P1 $\quad 14.75$ use

PL1W $\quad 10.56200695 \mathrm{~W}$

$400.1324710 \mathrm{MHz}$

F2 - Processing parameter

$\begin{array}{ll}\text { SI } & 32768 \\ \text { SF } & 400.1300136 \\ \text { MHz }\end{array}$

NDW

$\begin{array}{lll}\mathrm{SSB} & 0 & 0.30 \mathrm{~Hz} \\ \mathrm{LB} & 0 & \end{array}$

$\begin{array}{ll}\mathrm{GB} & 0 \\ \mathrm{PC} & \end{array}$
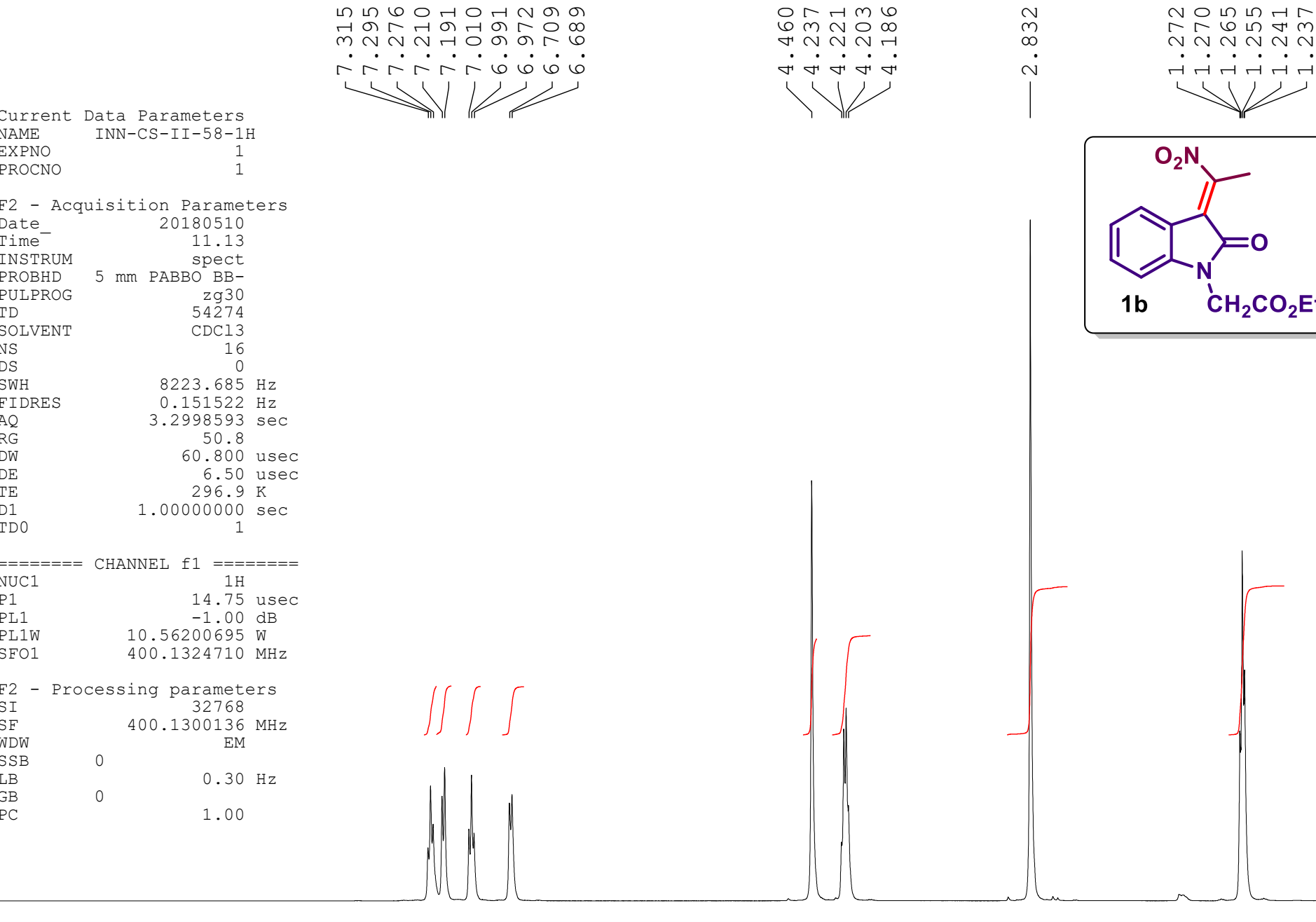

8

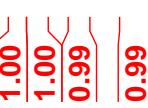

6

Figure S6. ${ }^{1} \mathrm{H}$ NMR (400 $\left.\mathrm{MHz}, \mathrm{CDCl}_{3}\right)$ Spectrum of $\mathbf{1 b}$
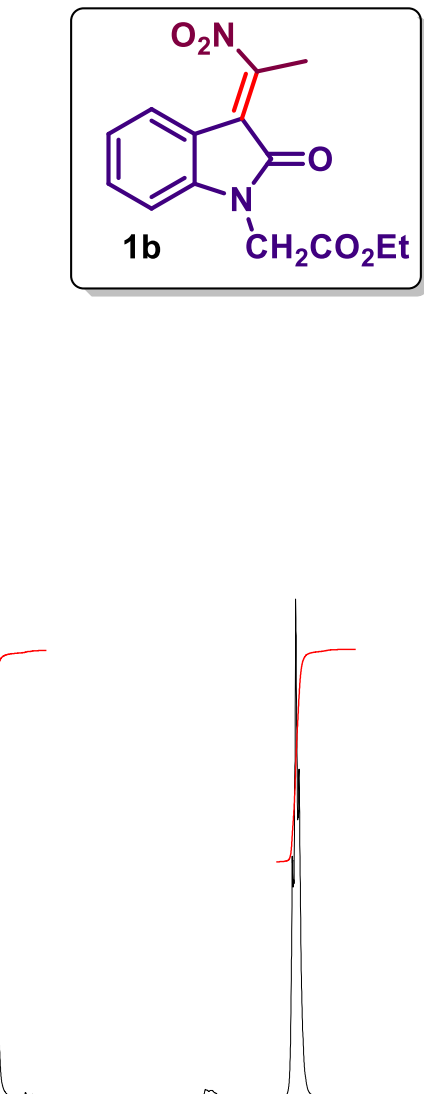

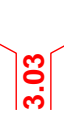

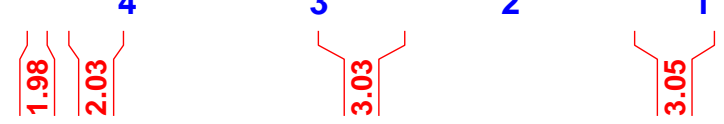

ְ. 


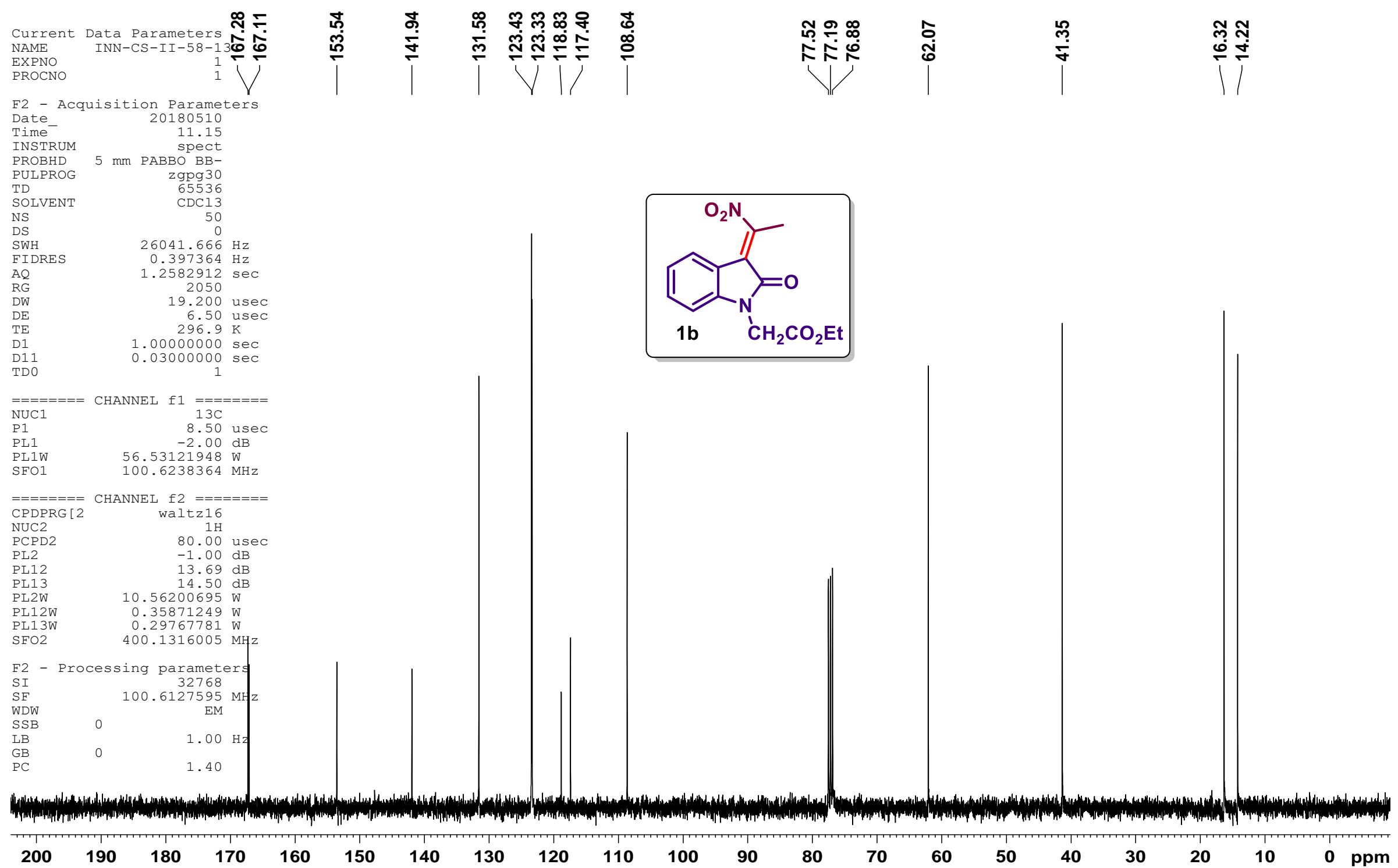

Figure S7. ${ }^{13} \mathrm{C}$ NMR $\left(100 \mathrm{MHz}, \mathrm{CDCl}_{3}\right)$ Spectrum of $\mathbf{1 b}$ 


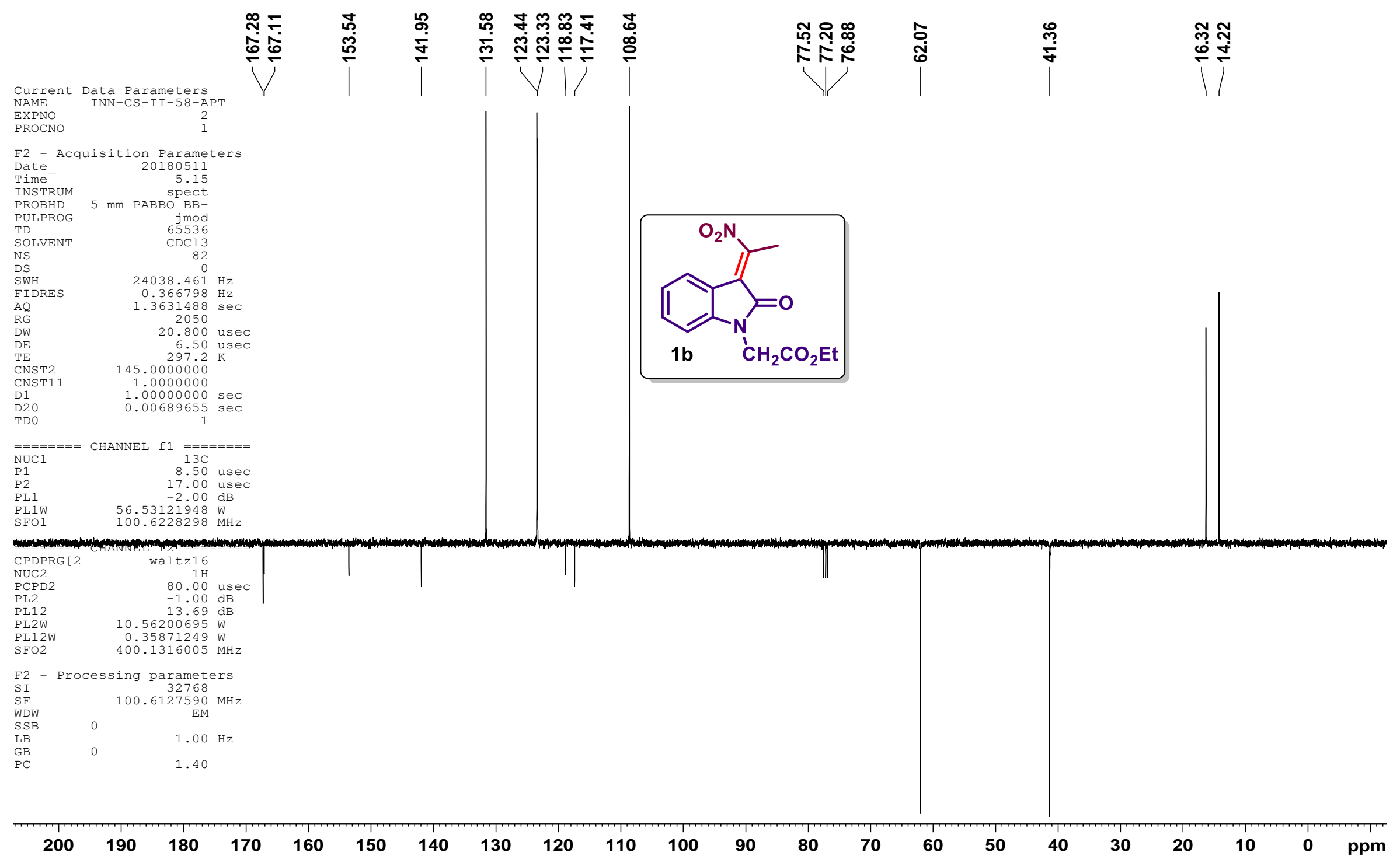

Figure S8. ${ }^{13} \mathrm{C}-\mathrm{APT}$ NMR $\left(100 \mathrm{MHz}, \mathrm{CDCl}_{3}\right)$ Spectrum of $\mathbf{1 b}$ 


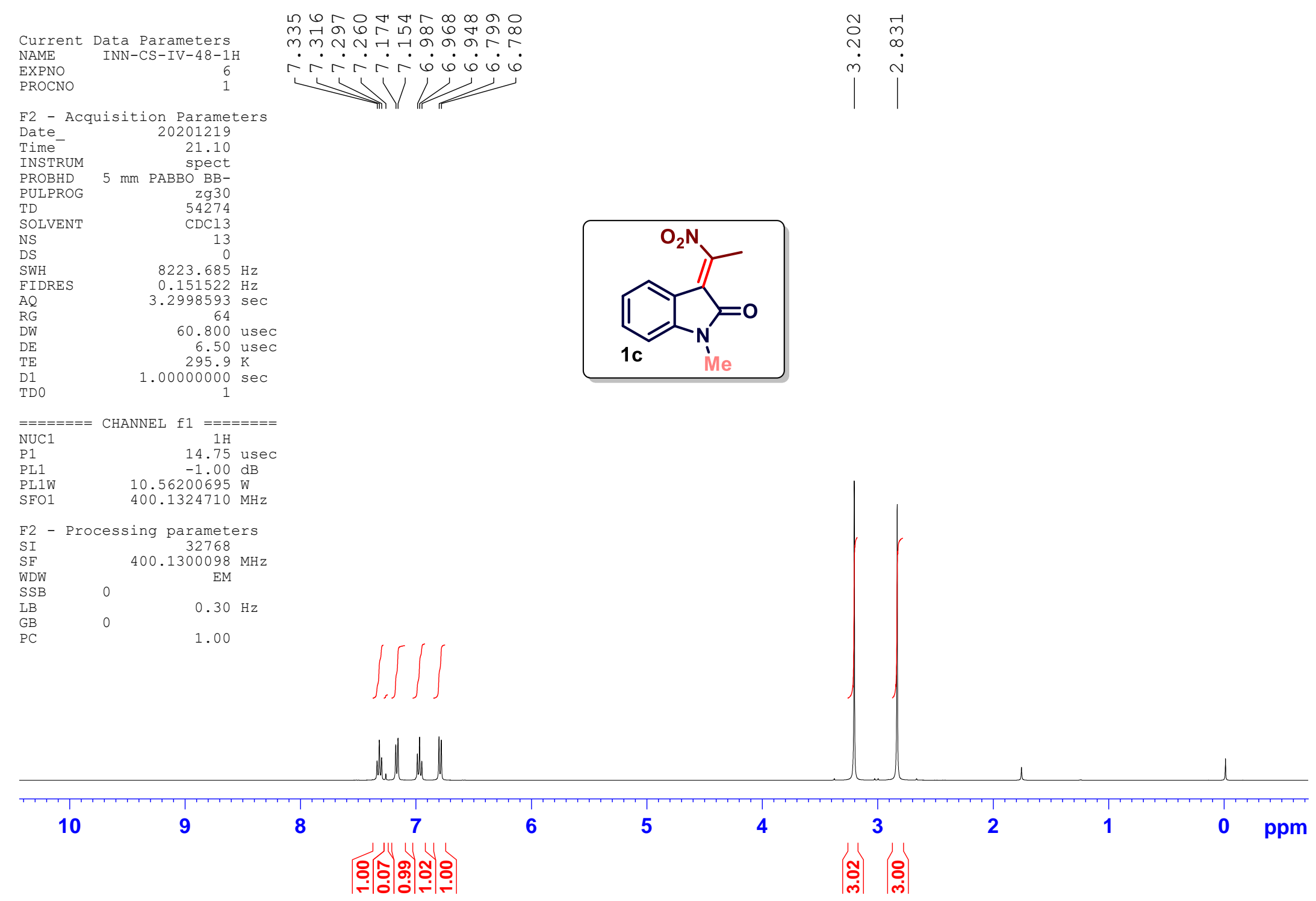

Figure S9. ${ }^{1} \mathrm{H}$ NMR (400 MHz, $\left.\mathrm{CDCl}_{3}\right)$ Spectrum of $\mathbf{1 c}$ 


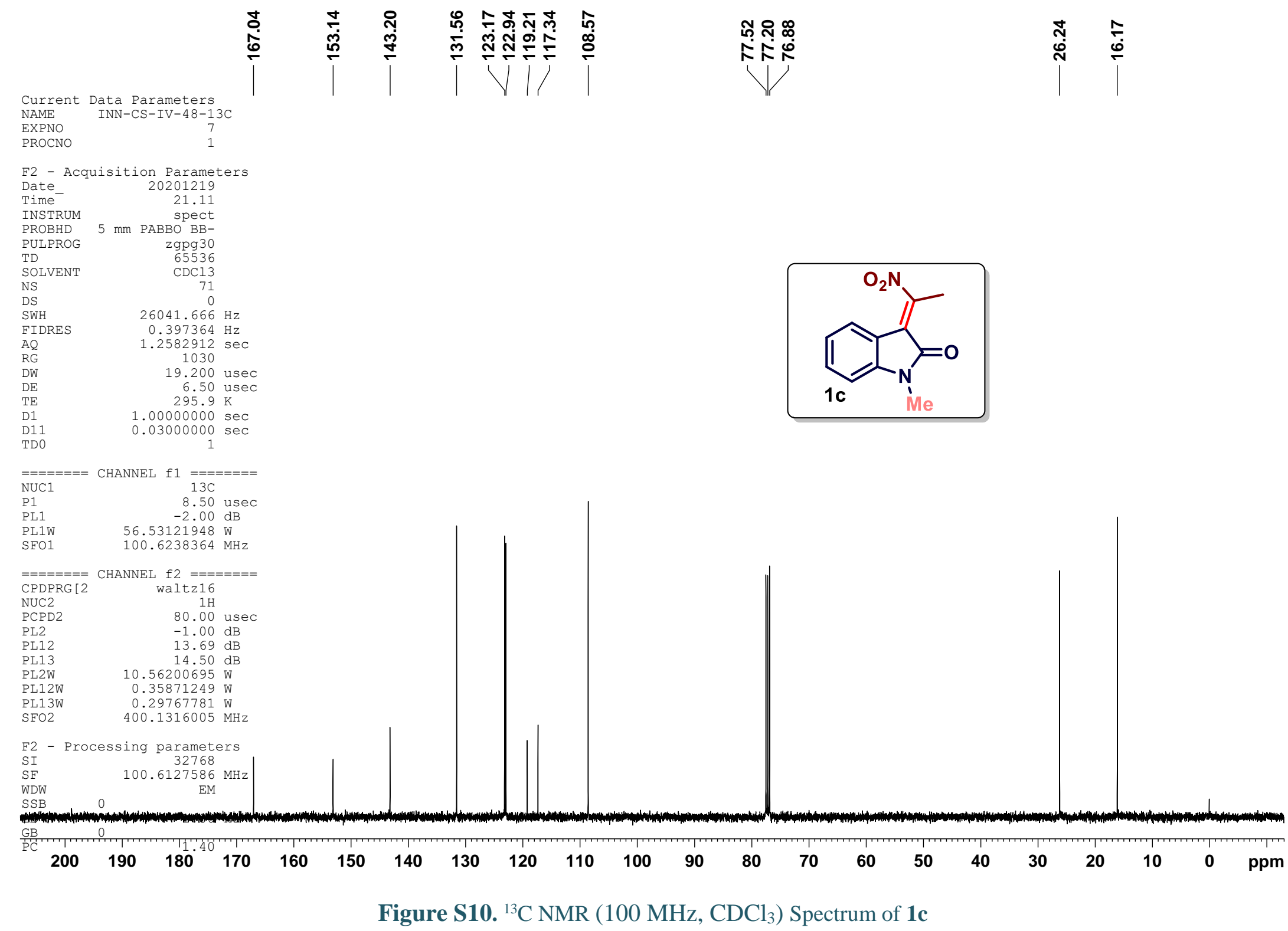




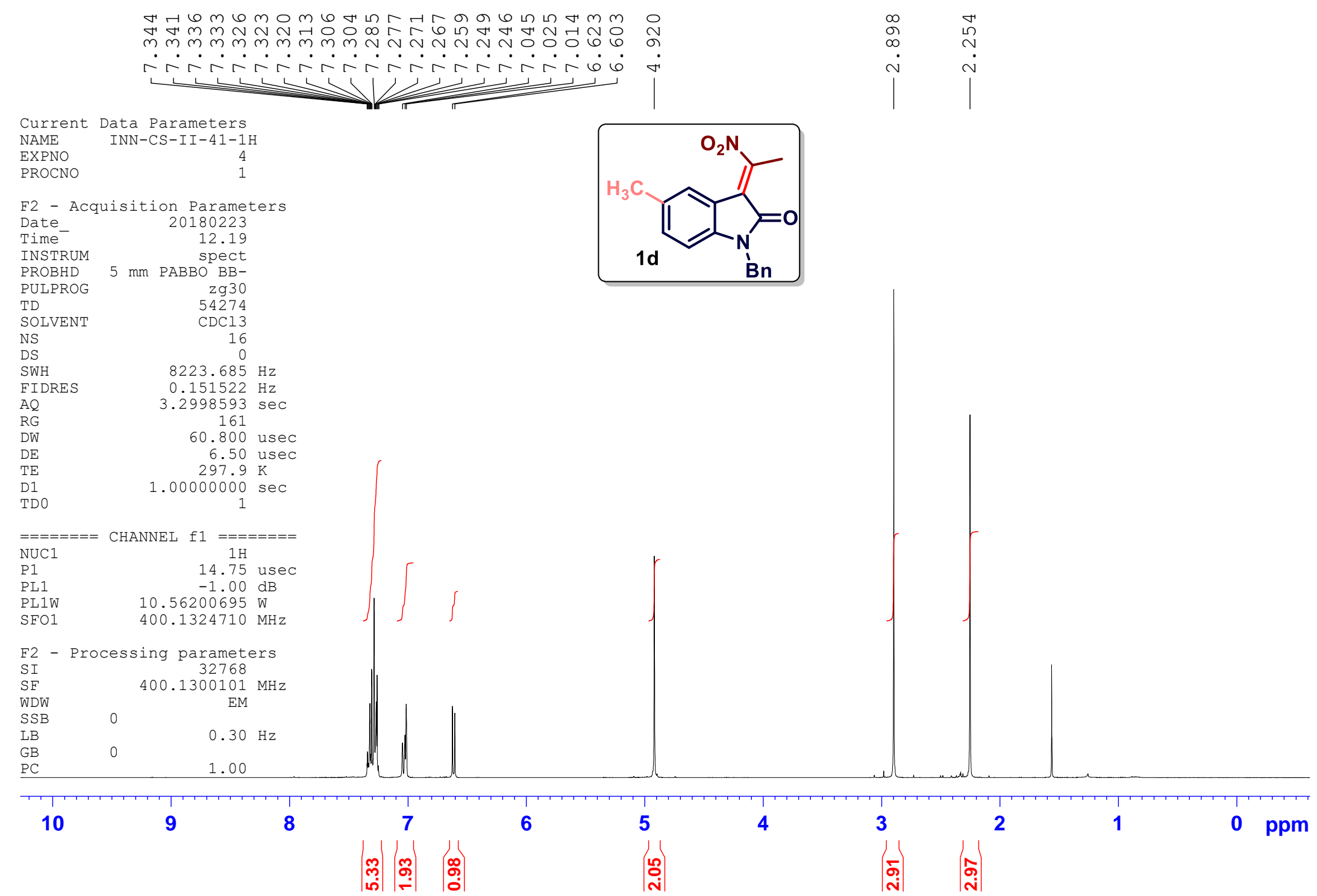

Figure S11. ${ }^{1} \mathrm{H}$ NMR (400 MHz, $\mathrm{CDCl}_{3}$ ) Spectrum of 1d 


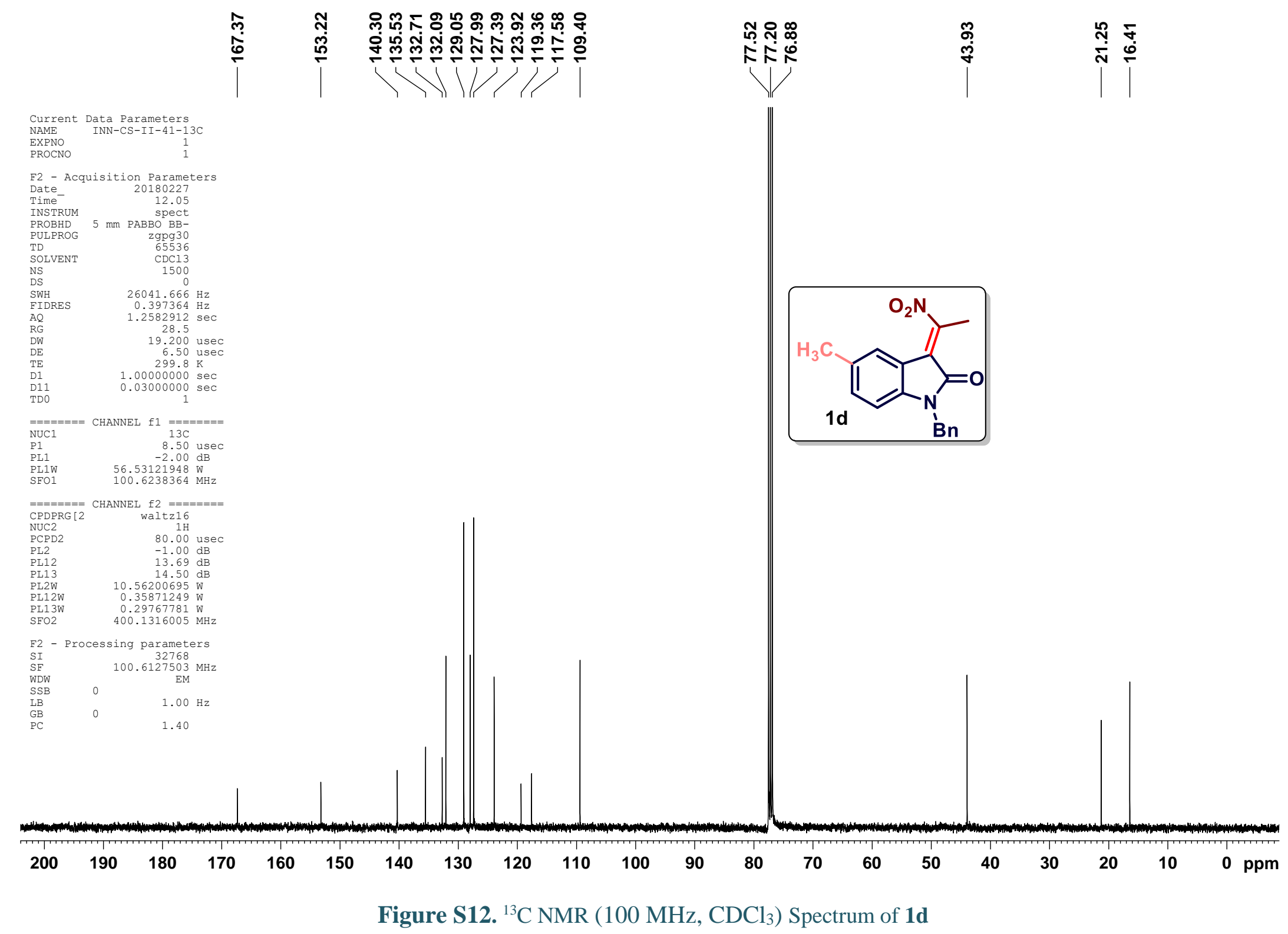


Current Data Parameters

Current Data Parameters

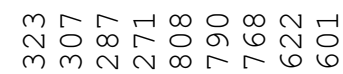

$\therefore \therefore م$

EXPNO

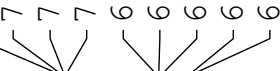

F2 - Acquisition Parameters

Date - 20180516

$\begin{array}{ll}14.08 \\ \text { INSTRUM } & \text { spect }\end{array}$

PROBHD $5 \mathrm{~mm}$ PABBO BB-

PULPROG

TD

NS
DS
SWH

FIDRES

AQ

RG

DE

D1 0

$=======$ CHANNEL $\mathrm{f}$

NUC1

P1 1

PL1W
SFO1

$\mathrm{zg} 30$
54274
$\mathrm{CDCl}$

0
0
$8223.685 \mathrm{~Hz}$

$0.151522 \mathrm{~Hz}$

$0.2998593 \mathrm{sec}$
71.8

60.800 usec

0.800 usec

F2 - Processing parameters

$\begin{array}{lr}\text { SI } & 32768 \\ \text { SF } & 400.1300102 \mathrm{MHz}\end{array}$

WDW $\quad 400.1300102$ MM

$\begin{array}{lll}\text { WSB } & 0 & 0.30 \mathrm{~Hz}\end{array}$

$\begin{array}{lll}\text { GB } & 0 & 1.00\end{array}$

PC

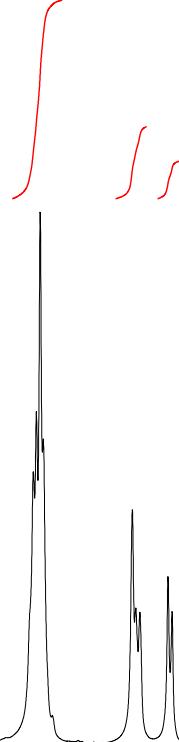

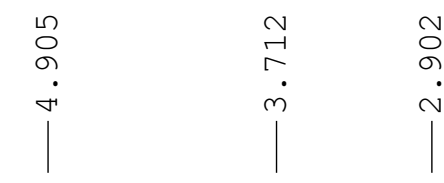

10

9

8

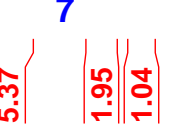

6

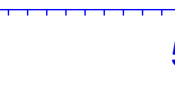

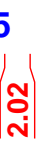

4

$\mid$

2

Figure S13. ${ }^{1} \mathrm{H}$ NMR (400 MHz, $\mathrm{CDCl}_{3}$ ) Spectrum of $\mathbf{1 e}$ 

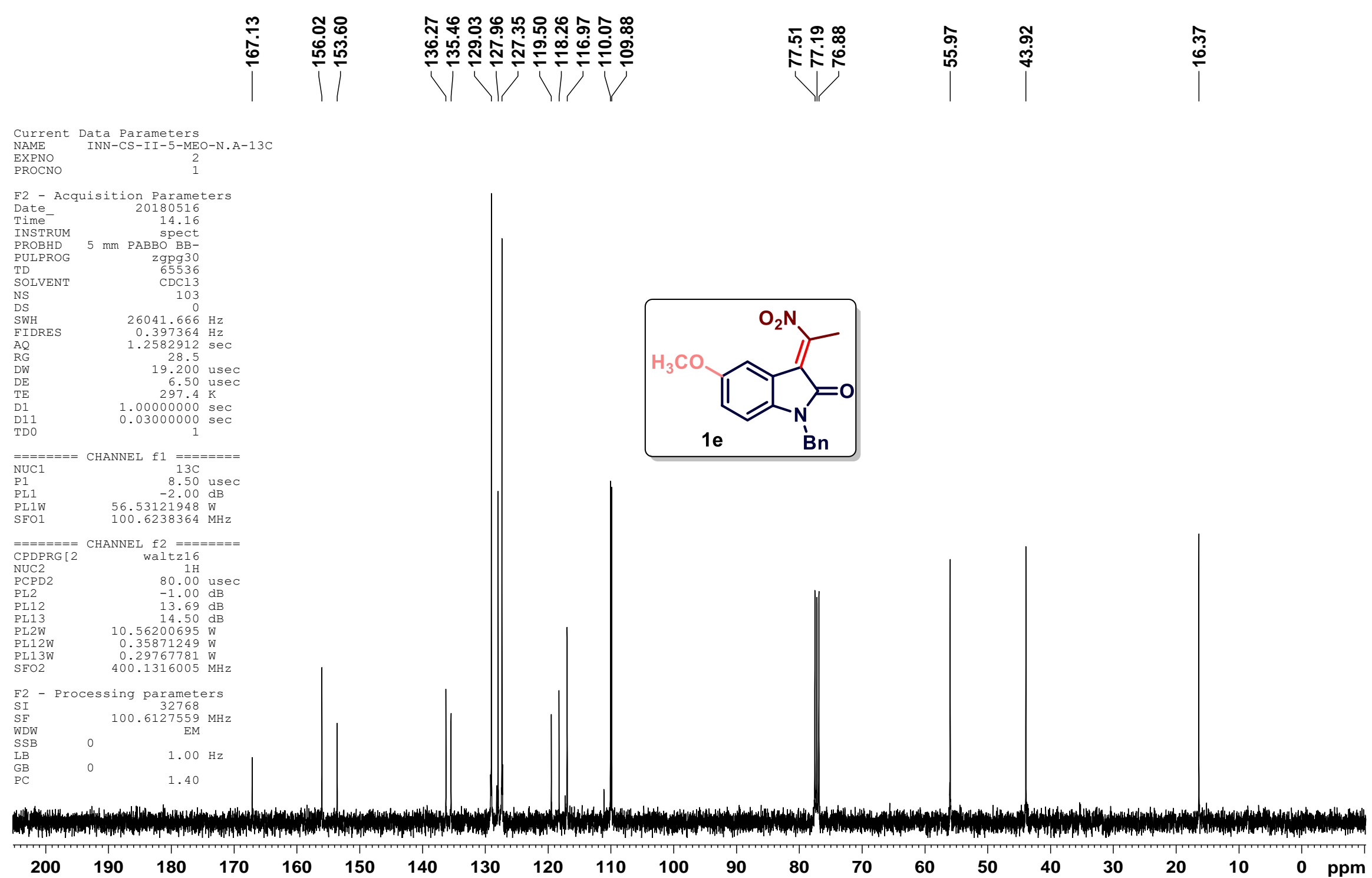

Figure S14. ${ }^{13} \mathrm{C}$ NMR $\left(100 \mathrm{MHz}, \mathrm{CDCl}_{3}\right)$ Spectrum of $\mathbf{1 e}$ 


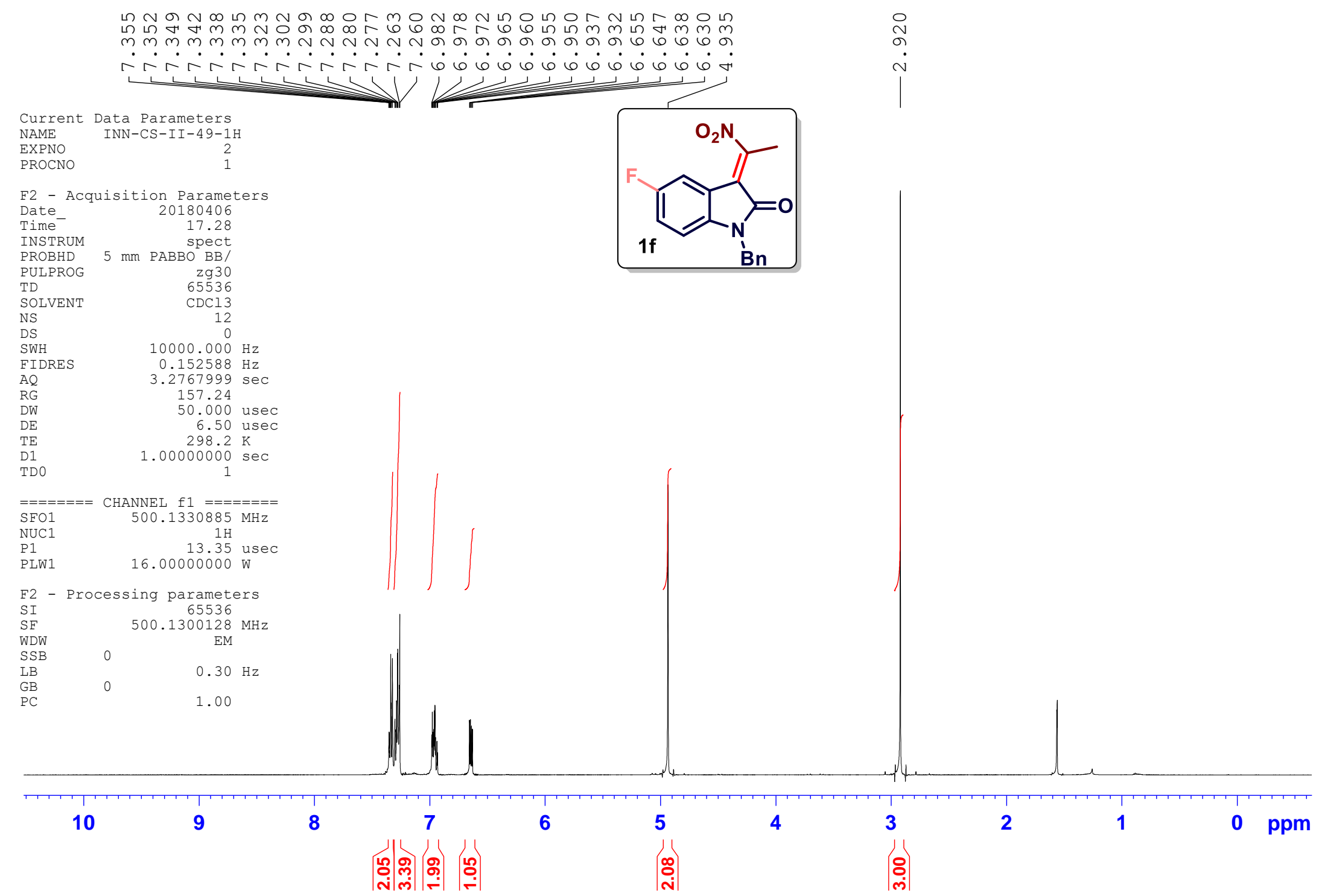

Figure S15. ${ }^{1} \mathrm{H}$ NMR $\left(500 \mathrm{MHz}, \mathrm{CDCl}_{3}\right)$ Spectrum of $\mathbf{1 f}$ 
Current Data Parameters
NAME
INN-CS-II-49-19F

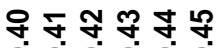

PROCNO

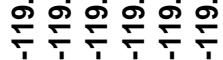

F2 - Acquisition Parameters

Date

INSTRUM

PROBHD

PULPROG
TD

NS
DS

SWH

$\mathrm{AQ}$
$\mathrm{RG}$

DW

TE
D1

20180429
0.24

$5 \mathrm{~mm}$ PABBO BB/

$\mathrm{BBO}$ BB/
$\mathrm{zgflqn}$
131072

$\mathrm{CDCl} 3$
50
0
113636.367

$0.866977 \mathrm{~Hz}$

4.400 usec

6.50 usec
$297.4 \mathrm{~K}$

$1.0000000 \mathrm{sec}$

$=======$ CHANNEL $\mathrm{f} 1 \mathrm{1}=======$
SFO1
NUC1

$\begin{array}{lr}\text { P1 } & 19 \mathrm{~F} \\ & 19.75 \text { used }\end{array}$

F2 - Processing parameter

FI - Processing parameters

WDW $\quad$ EM

$\begin{array}{lll}\mathrm{LSB} & 0 & 0.30 \mathrm{~Hz}\end{array}$

PC 1.00
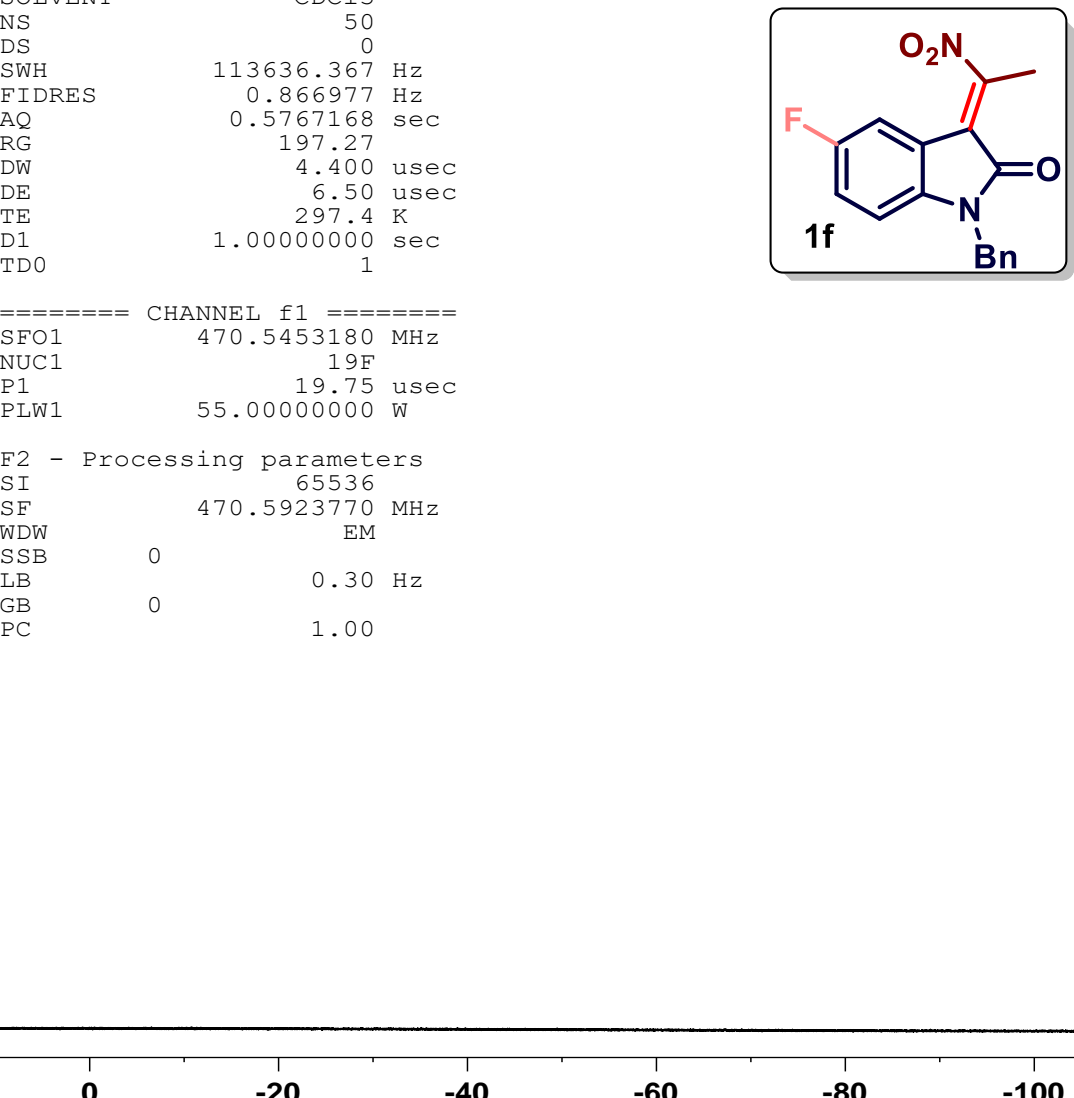

$-40$

$-60$

$-80$

$-100$

$-140$

$-160$

$-180$

Figure S16. ${ }^{19} \mathrm{~F}$ NMR (470 MHz, $\left.\mathrm{CDCl}_{3}\right)$ Spectrum of $\mathbf{1 f}$ 


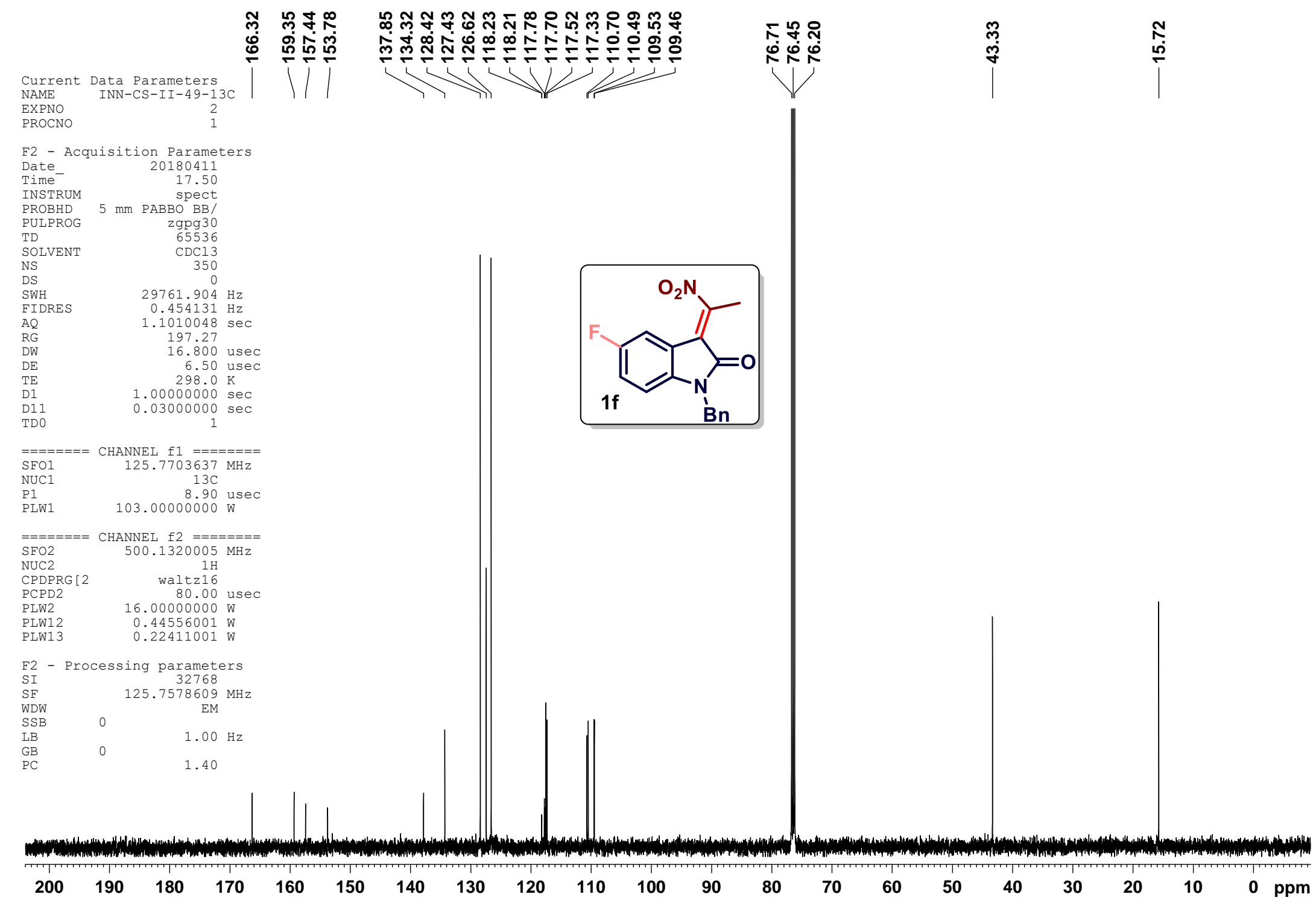

Figure S17. ${ }^{13} \mathrm{C}$ NMR $\left(125 \mathrm{MHz}, \mathrm{CDCl}_{3}\right)$ Spectrum of $\mathbf{1 f}$ 


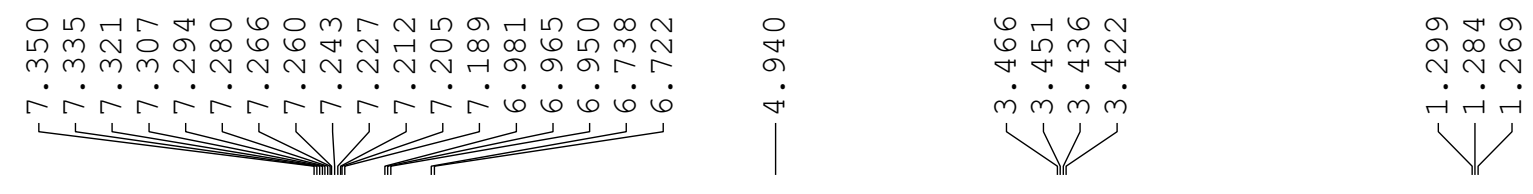

Current Data Parameters

NAME INN-CS-II-31-Ethyl-N.A-1H

EXPNO

1

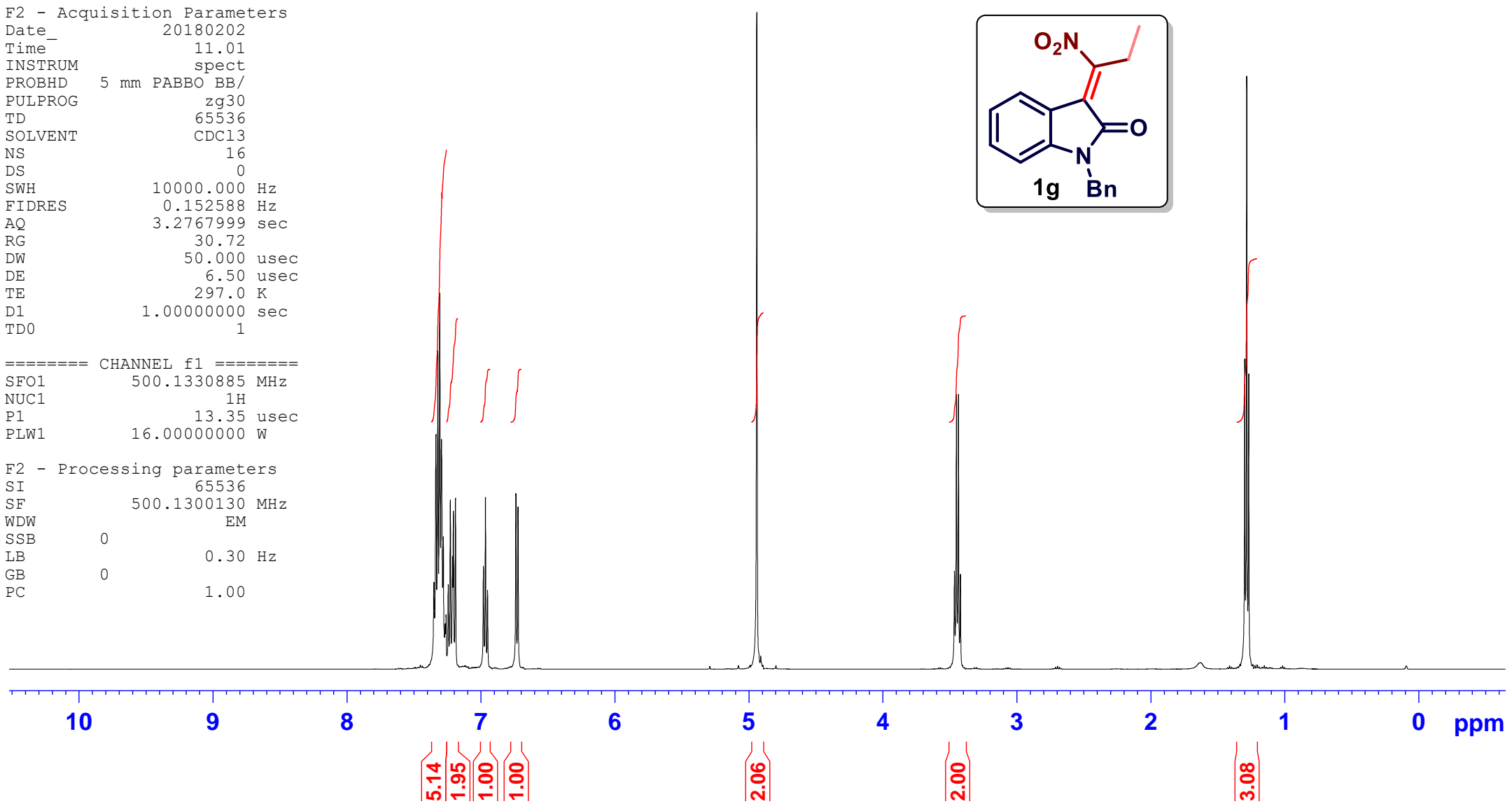

Figure S18. ${ }^{1} \mathrm{H}$ NMR $\left(500 \mathrm{MHz}, \mathrm{CDCl}_{3}\right)$ Spectrum of $\mathbf{1 g}$ 


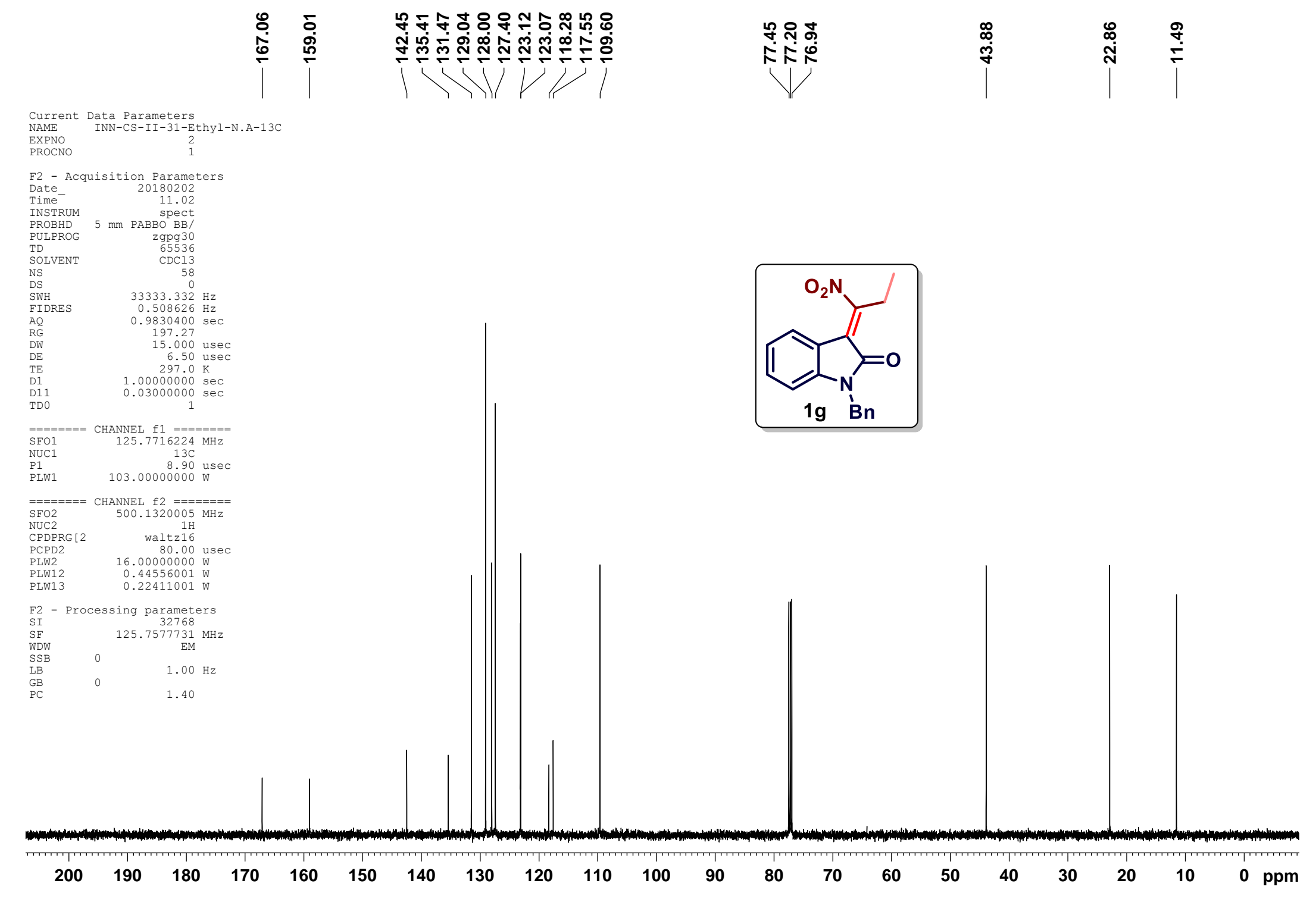

Figure S19. ${ }^{13} \mathrm{C}$ NMR $\left(125 \mathrm{MHz}, \mathrm{CDCl}_{3}\right)$ Spectrum of $\mathbf{1 g}$ 


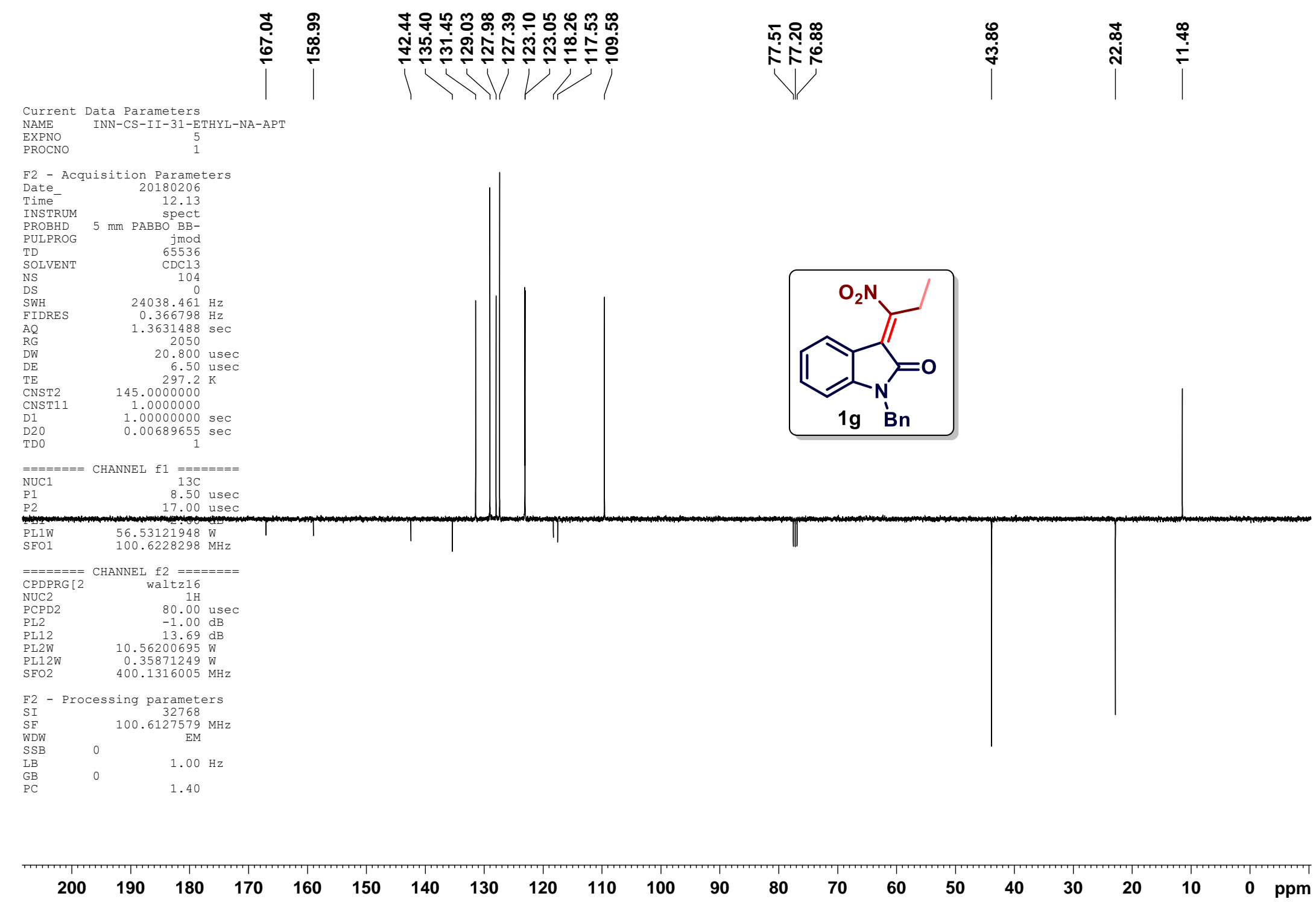

Figure S20. ${ }^{13} \mathrm{C}$-APT NMR (100 MHz, $\left.\mathrm{CDCl}_{3}\right)$ Spectrum of $\mathbf{1 g}$ 


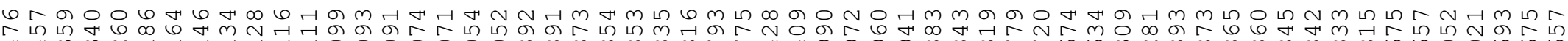
F $\mathrm{m} m \mathrm{~N}$

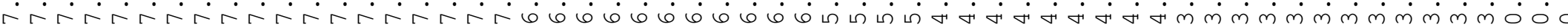

Current Data Parameters

NAME

EXPNO

F2 - Acquisition Parameters

Date_

Time $\quad 16.40$

PROBHD $5 \mathrm{~mm}$ PABBO BB-

$\begin{array}{lr}\text { PULPROG } & \text { Zg30 } \\ \text { TD } & 5427\end{array}$

TD
SOLVENT

NS

CDC13

9
0

SWH $\quad 8223.685 \mathrm{~Hz}$

FIDRES $\quad 0.151522 \mathrm{~Hz}$

AQ $\quad 3.2998593 \mathrm{sec}$

RG

$\begin{array}{lr}\text { DW } & 60.800 \text { usec } \\ \text { DE } & 6.50 \text { usec }\end{array}$

$T E$

TD $1.00000000 \mathrm{sec}$

$=======$ CHANNEL $\mathrm{f} 1 \mathrm{l}======$

$\mathrm{NUC1}$

$\begin{array}{ll}\text { PL1 } & -1.00 \mathrm{~dB} \\ \text { PL1W } & 10.56200695 \mathrm{~W} \\ \text { SFO1 } & 400.1324710 \mathrm{MHz}\end{array}$

F2 - Processing parameters

$\begin{array}{lr}\text { SI } & 32768 \\ \text { SF } & 400.1300100 \mathrm{MHz}\end{array}$

$\begin{array}{lll}\text { SF } & 400.1300100 \\ \text { WDW } & \end{array}$

$\begin{array}{lcc}\text { WDW } & \text { EM } \\ \text { SSB } & 0 & 0.30 \mathrm{~Hz} \\ \text { LB } & & \end{array}$

\begin{tabular}{lll|} 
LB & $0.30 \mathrm{~Hz}$ \\
$\mathrm{~GB}$ & 0 & 0.30 \\
PC & &
\end{tabular}

10

9

8

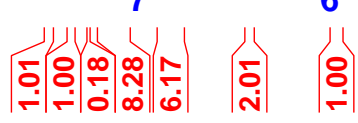

5

( ) $1+1$

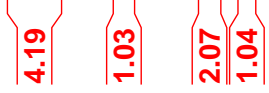

3

(ָ)

Figure S21. ${ }^{1} \mathrm{H}$ NMR (400 $\mathrm{MHz} \mathrm{CDCl}_{3}$ ) Spectrum of 3a 


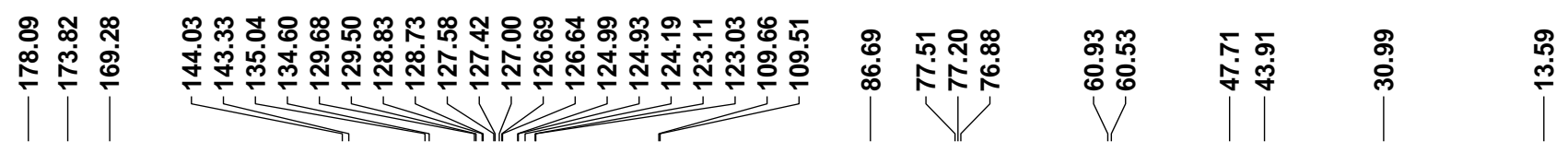

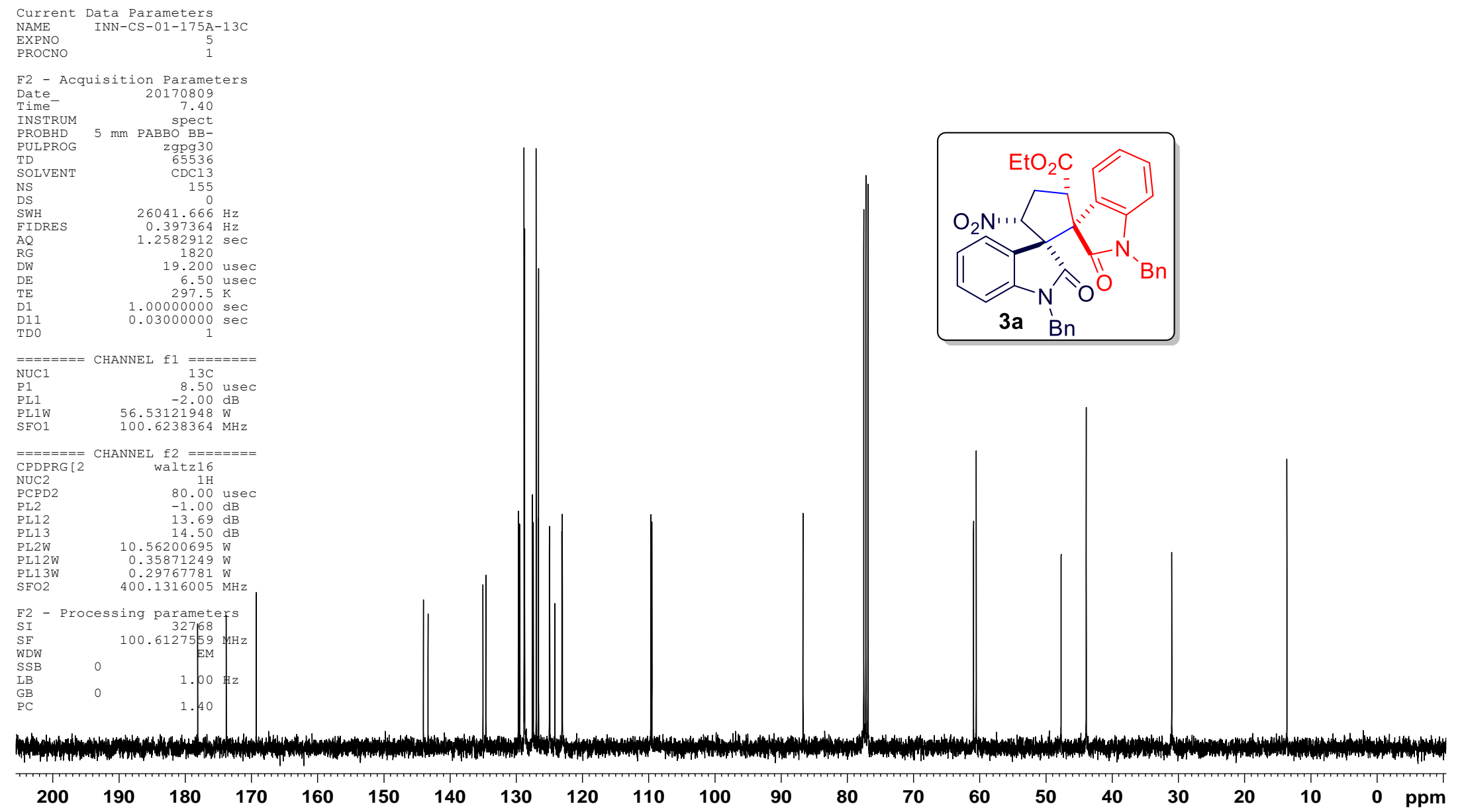

Figure S22. ${ }^{13} \mathrm{C}$ NMR $\left(100 \mathrm{MHz}, \mathrm{CDCl}_{3}\right)$ Spectrum of 3a 

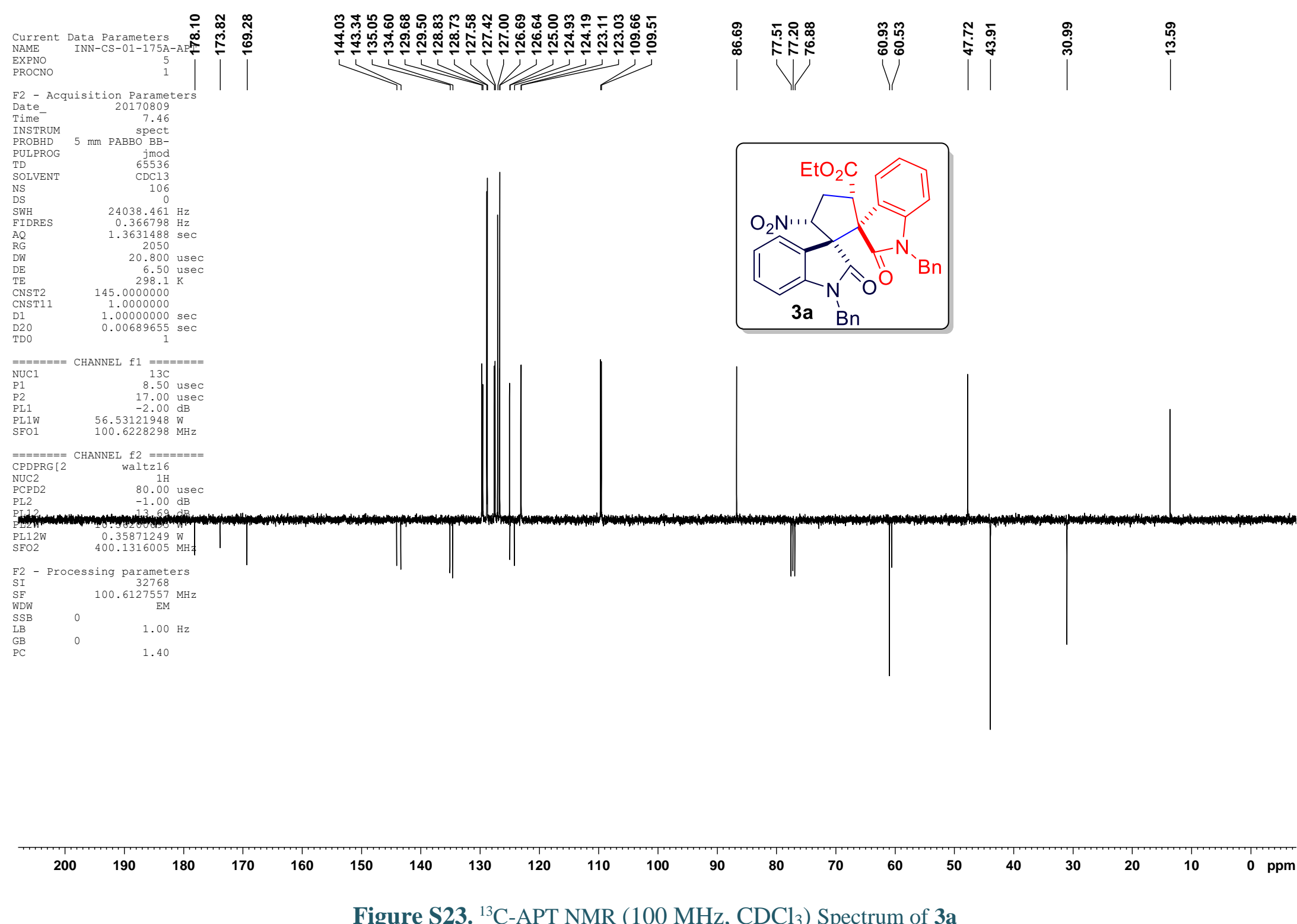


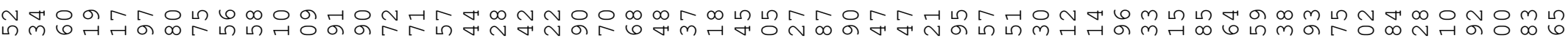

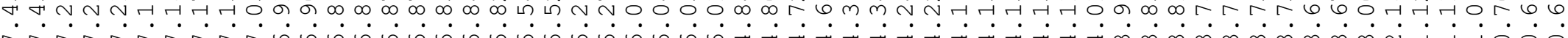

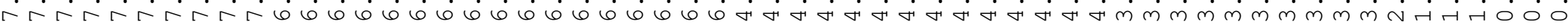

Current Data Parameters

INN-CS-II-62-1

EXPNO

F2 - Acquisition Parameter

Date_ 20180429

Time 11.06

$5 \mathrm{~mm}$ PABBO BB-

PROBHD

TO 30
TD

SOLVEN

NS

$\begin{array}{lr} & 0 \\ \text { SWH } & 8223.685 \mathrm{~Hz}\end{array}$

$\begin{array}{lr}\text { FIDRES } & 0.151522 \mathrm{~Hz} \\ \mathrm{AO} & 3.2998593 \mathrm{sec}\end{array}$

$\begin{array}{rr}\text { AQ } & 3.2998593 \mathrm{sec} \\ \text { RG } & 71.8\end{array}$

$\begin{array}{lr}\text { RG } & 71.8 \\ \text { DW } & 60.800 \text { usec }\end{array}$

DE $\quad 6.50$ use

$\begin{array}{lr}\text { TE } & 296.5 \mathrm{~K} \\ \text { D1 } & 1.00000000 \mathrm{sec}\end{array}$

TDO

$=======$ CHANNEL $\mathrm{f} 1 \begin{gathered}======== \\ 1 \mathrm{H}\end{gathered}$
NUC1

$\mathrm{P} 1$

PL1 14.75 usec

PL1W $\quad 10.56200695 \mathrm{~W}$

$400.1324710 \mathrm{MHz}$

F2 - Processing parameters

$\begin{array}{lr}\text { SI } & 32768 \\ \text { SF } & 400.1300102 \mathrm{MHz}\end{array}$

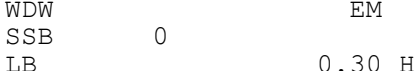

$\begin{array}{lll}\mathrm{LB} & 0.30 \mathrm{~Hz}\end{array}$

$\begin{array}{ll}\mathrm{GB} & \mathrm{PC} \\ \end{array}$

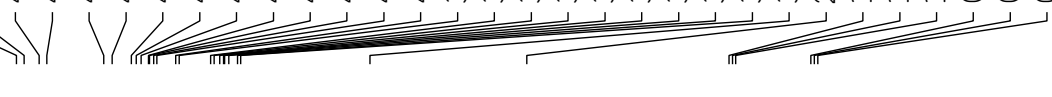

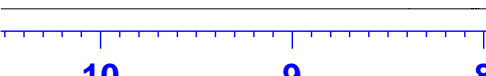

8
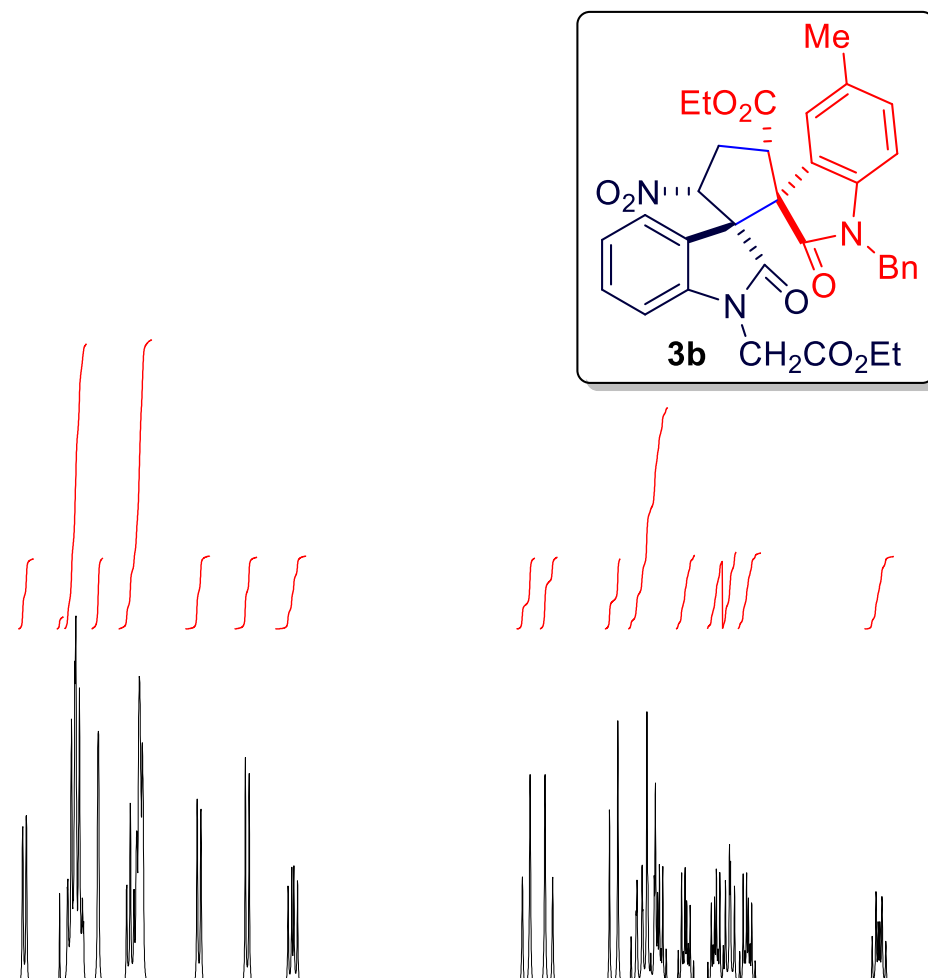

3b $\mathrm{CH}_{2} \mathrm{CO}_{2} \mathrm{Et}$
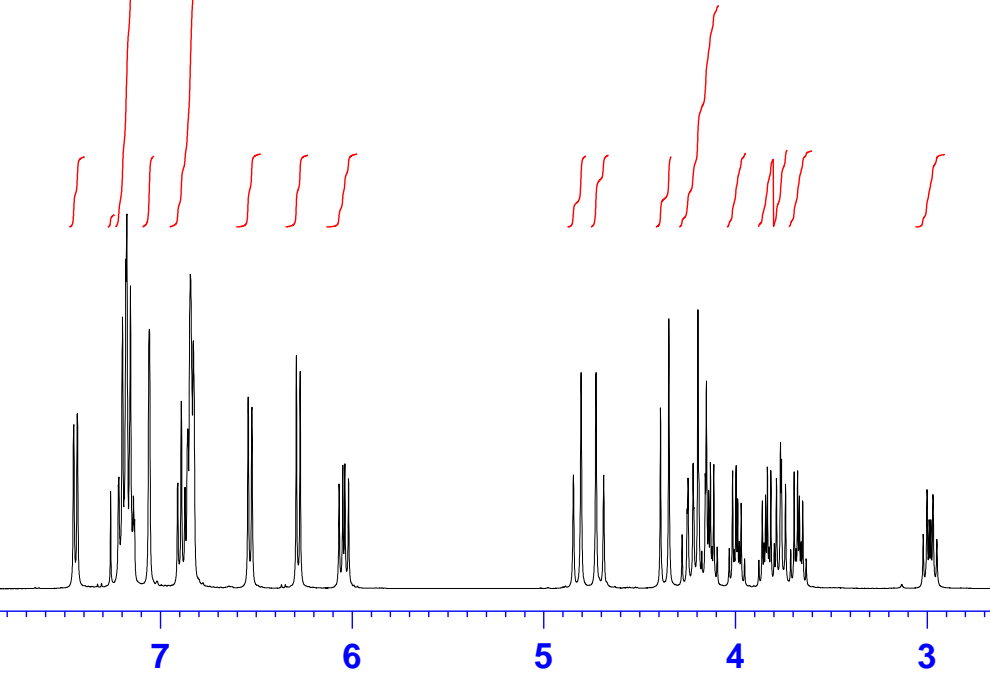

7

6

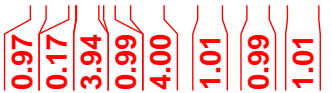

3

(m)

|

2

Figure S24. ${ }^{1} \mathrm{H}$ NMR (400 $\mathrm{MHz}, \mathrm{CDCl}_{3}$ ) Spectrum of $\mathbf{3 b}$ 

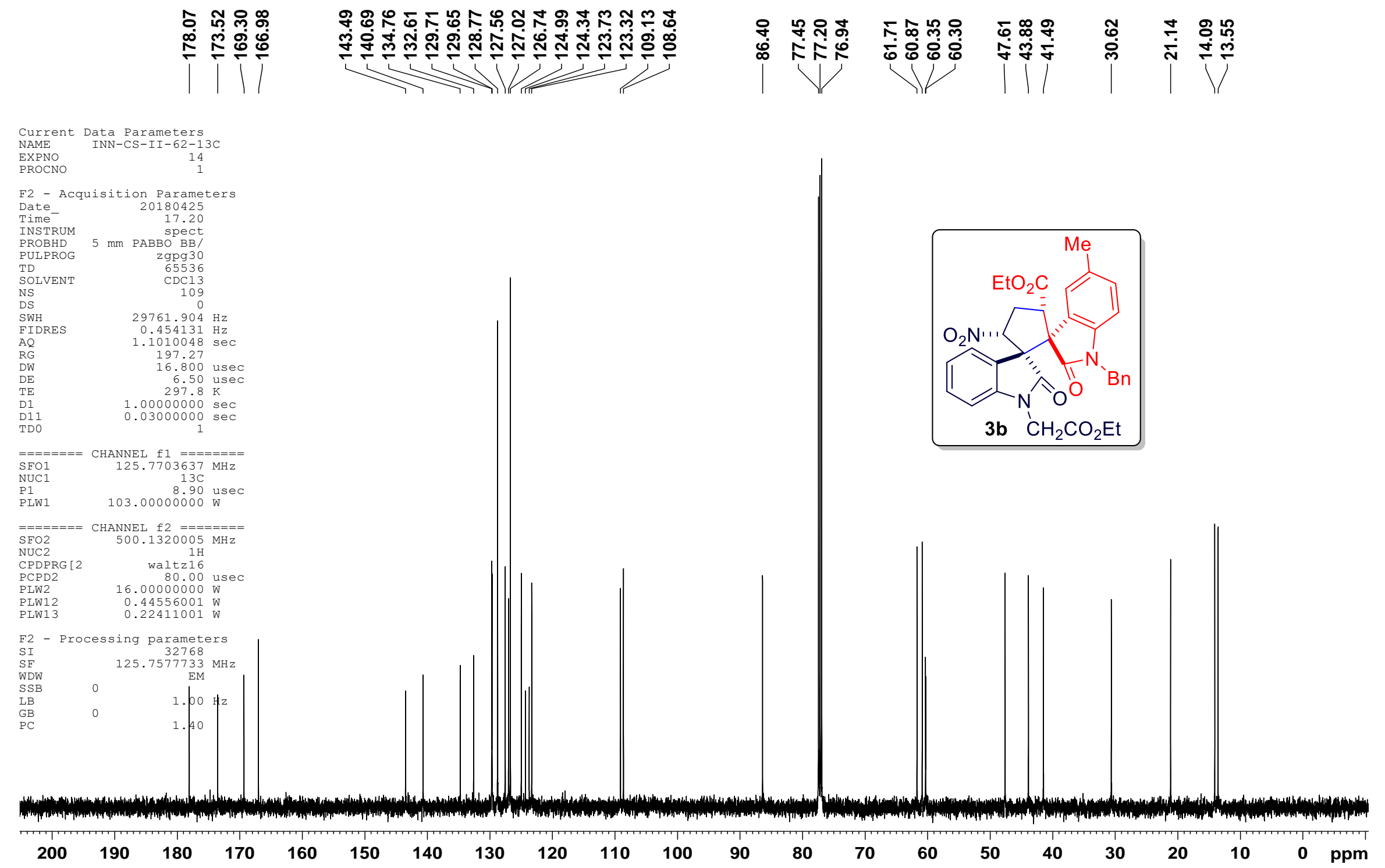

Figure $\mathbf{S 2 5} .{ }^{13} \mathrm{C} \mathrm{NMR}\left(125 \mathrm{MHz}, \mathrm{CDCl}_{3}\right)$ Spectrum of $\mathbf{3 b}$ 


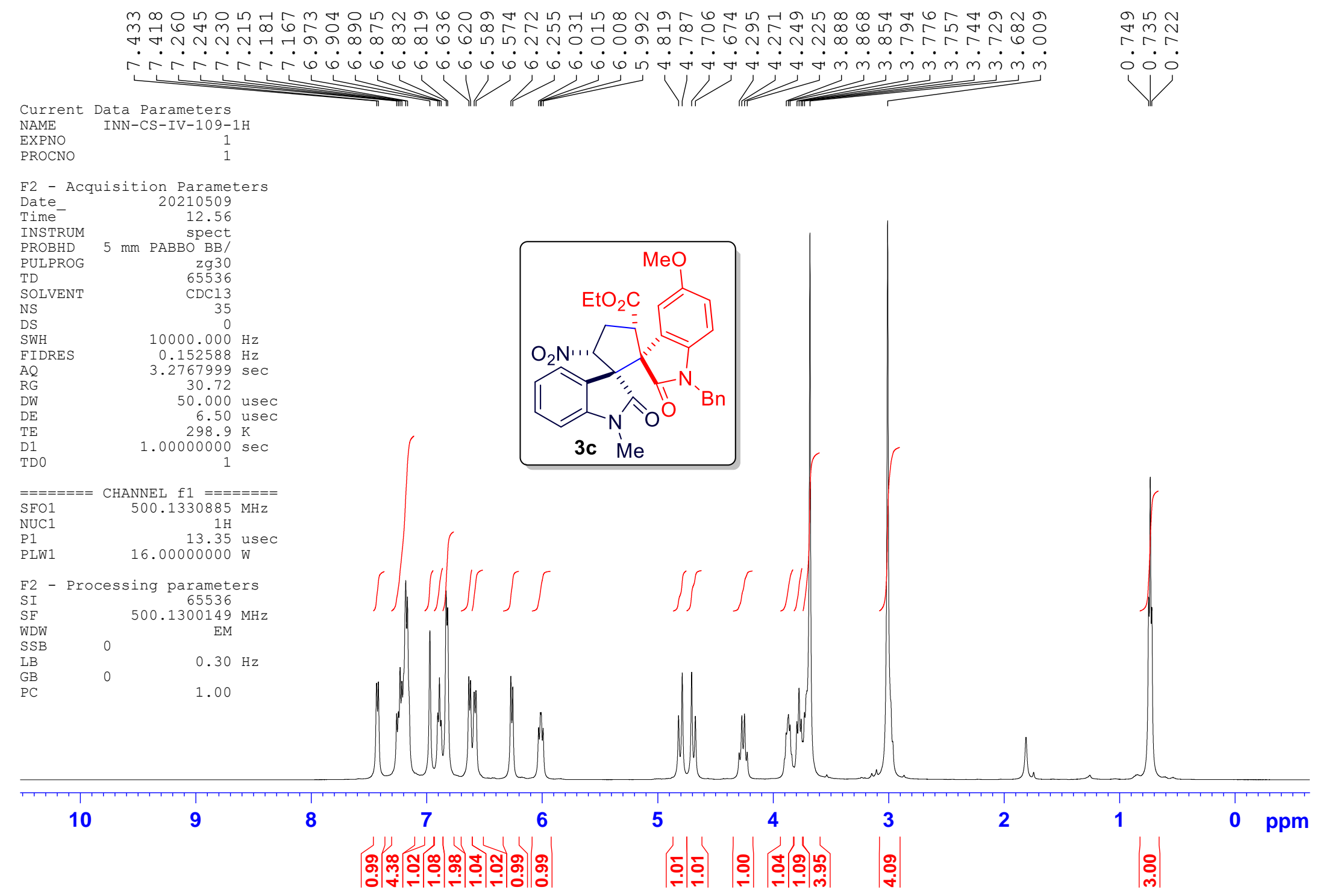

Figure S26. ${ }^{1} \mathrm{H}$ NMR $\left(500 \mathrm{MHz}, \mathrm{CDCl}_{3}\right)$ Spectrum of $\mathbf{3 c}$ 


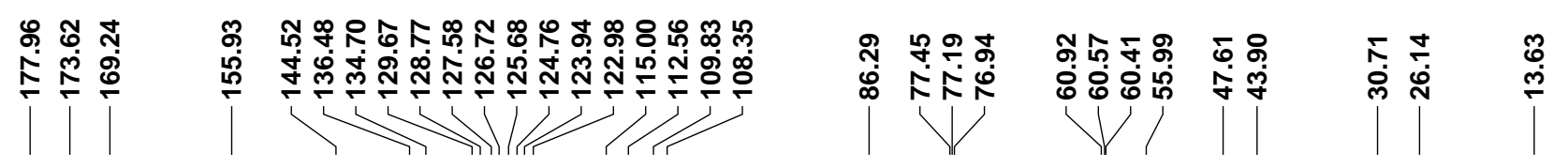

Current Data Parameters
NAME INN-CS-IV-109-13C

EXPNO
PROCNO

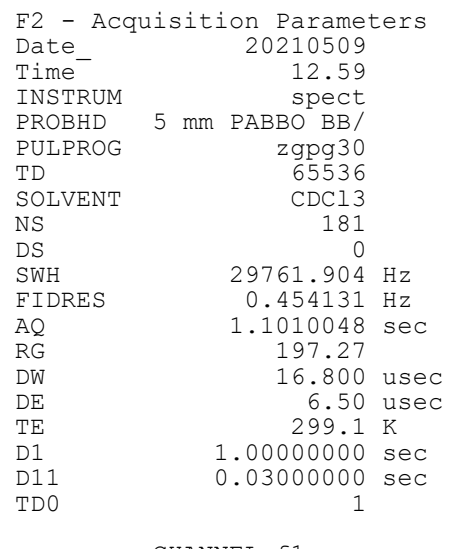

$=======$ CHANNEL $\mathrm{fl}========$
$\mathrm{SFO1}$

$\begin{array}{ll}\text { SFO1 } & 125.7703637 \mathrm{MHz}\end{array}$

P1 $\quad 8.90$ use

$\begin{array}{ll}======= & \text { CHANNEL } \mathrm{f} 2====== \\ \text { SFO2 } & 500.1320005 \mathrm{MHz}\end{array}$

NUC2
CPDPRG [2

$\begin{array}{lr}\text { CPDPRG [2 } & \text { waltz16 } \\ \text { PCPD2 } & 80.00 \text { usec } \\ \text { PLW2 } & 16.00090000 \mathrm{~W}\end{array}$

$\begin{array}{lr}\text { PLW2 } & 16.00090000 \mathrm{~W} \\ \text { PLW12 } & 0.44506001 \mathrm{~W} \\ \text { PLW13 } & 0.2241001\end{array}$

F2 - Processing palameters

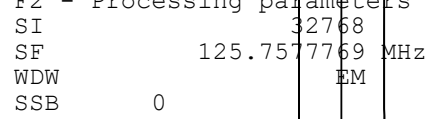

PC

$\begin{array}{lllllllllllllllllllllll}200 & 190 & 180 & 170 & 160 & 150 & 140 & 130 & 120 & 110 & 100 & 90 & 80 & 70 & 60 & 50 & 40 & 30 & 20 & 10 & 0 & \text { ppm }\end{array}$

Figure S27. ${ }^{13} \mathrm{C}$ NMR (125 $\left.\mathrm{MHz} \mathrm{CDCl}_{3}\right)$ Spectrum of $\mathbf{3 c}$

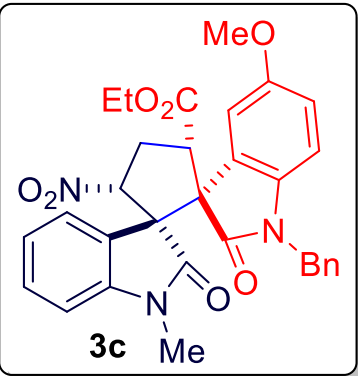


คุำ

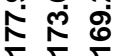

Current Data Parameters NAME INN-CS-IV-109-APT EXPNO

F2 - Acquisition Parameters

Date

INSTRUM

PROBHD

$T$ TD

SOLVE
NS

SWH

FIDRES

AQ

$\mathrm{DW}$
$\mathrm{DE}$

TE

CNST2 2

D1
D20

PLW1

mm PABBO BB/

jmod
65536
CDC13
123

$29761.904 \mathrm{~Hz}$

$0.454131 \mathrm{~Hz}$

$.1010048 \mathrm{sec}$
197.27

16.800 usec

6.50 usec

145.0000000

1.0000000

$0.00689655 \mathrm{sec}$

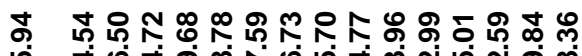

กิ ปั

ำำ กำกำ

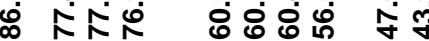

VI I

i

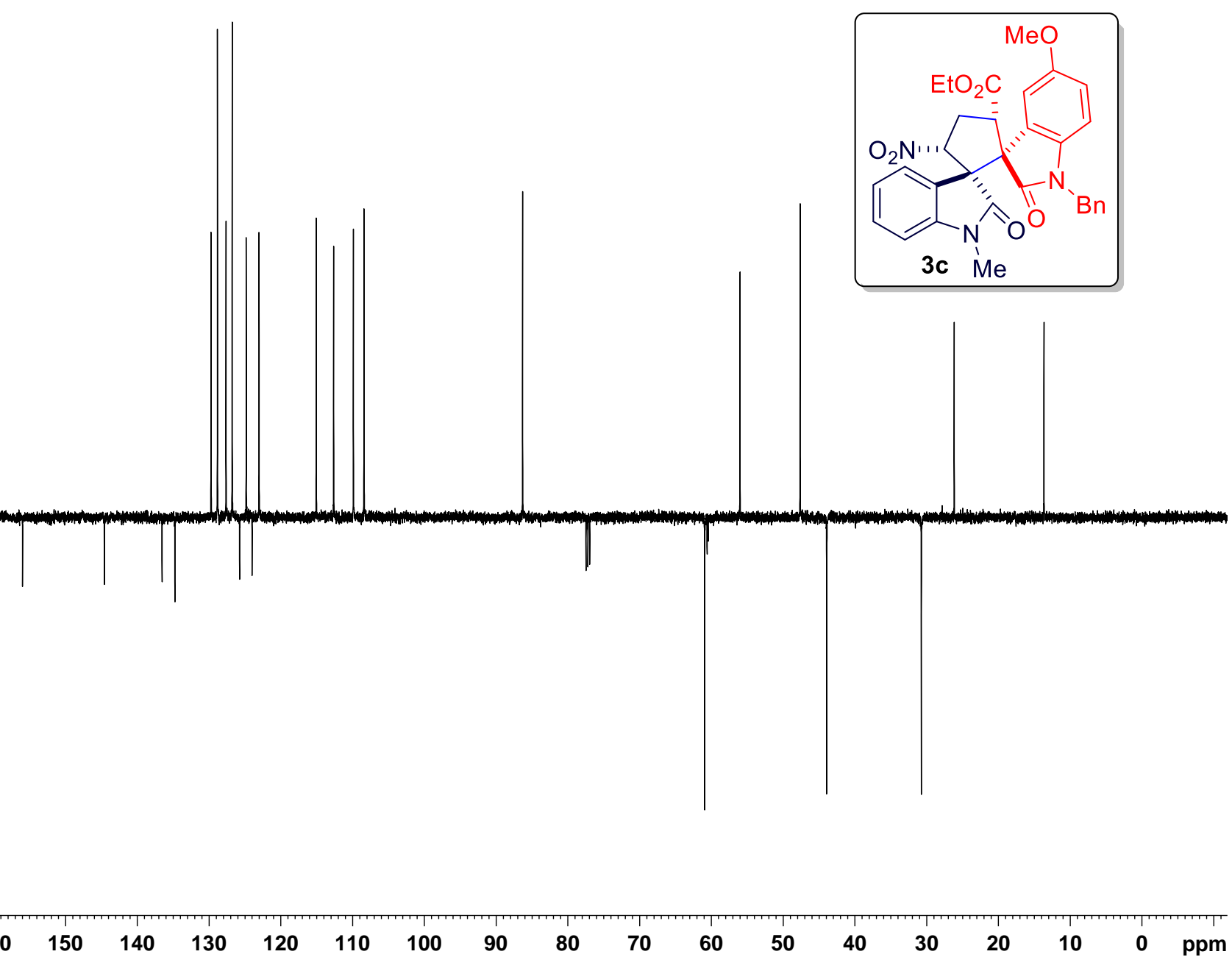

Figure S28. ${ }^{13} \mathrm{C}$-APT NMR $\left(125 \mathrm{MHz}, \mathrm{CDCl}_{3}\right)$ Spectrum of $\mathbf{3 c}$ 


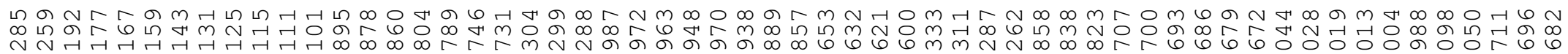

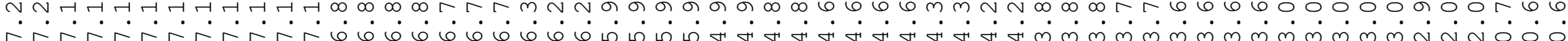
Curameters Current Data Parameters NAME INN-CS-II-43-1H

EXPNO

F2 - Acquisition Parameters

Date_ $\quad 20210117$

INSTRUM spect

PROBHD $5 \mathrm{~mm}$ PABBO BB/

PULPROG 2 zg30

$\begin{array}{ll}\text { TD } & 65536 \\ \text { SOLVENT } & \text { CDC13 }\end{array}$

NS

$\begin{array}{lr}\text { DS } & 0 \\ \text { SWH } & 10000.000 \mathrm{~Hz}\end{array}$

FIDRES $\quad 0.152588 \mathrm{~Hz}$

$\begin{array}{lr}A Q & 3.2767999 \\ R G & 53.37\end{array}$

DW $\quad 50.000$ usec

$\begin{array}{ll}\mathrm{DE} & 6.50 \text { usec } \\ \mathrm{TE} & 299.3 \mathrm{~K}\end{array}$

D1 $1.00000000 \mathrm{sec}$

$=======$ CHANNEL $\mathrm{f} 1 \mathrm{l}========$ SFO1 $500.1330885 \mathrm{MHz}$

NUC1 $1 \mathrm{H}$

P1 13.35 usec

PLW1 $16.00000000 \mathrm{~W}$

F2 - Processing parameters

SI $\quad 500.1305536$

SF $\quad 500.1300144 \mathrm{MH}$

$\begin{array}{lll}\text { SSB } & 0 & 0.30 \mathrm{~Hz}\end{array}$

$\begin{array}{ll}\mathrm{GB} & 0 \\ \mathrm{PC} & \end{array}$

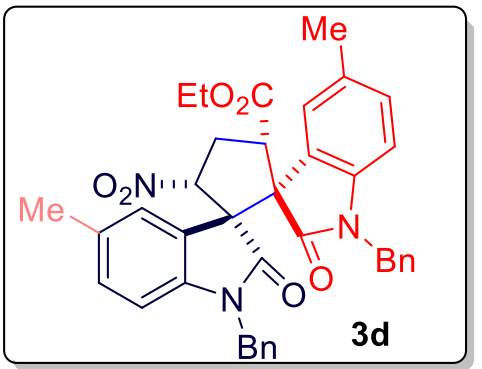

1.00

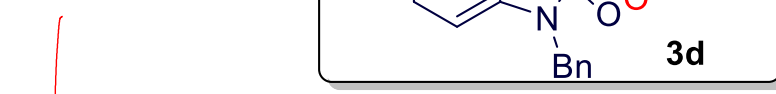

9

8

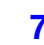

7

5

4

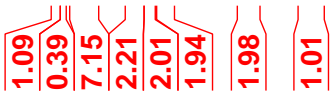

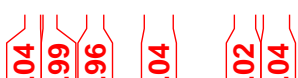

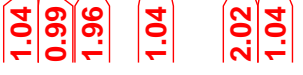

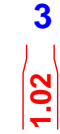

\begin{tabular}{l|l}
2 \\
0 \\
0 \\
0
\end{tabular}

0 ppm

Figure S29. ${ }^{1} \mathrm{H}$ NMR $\left(500 \mathrm{MHz}, \mathrm{CDCl}_{3}\right)$ Spectrum of $\mathbf{3 d}$ 


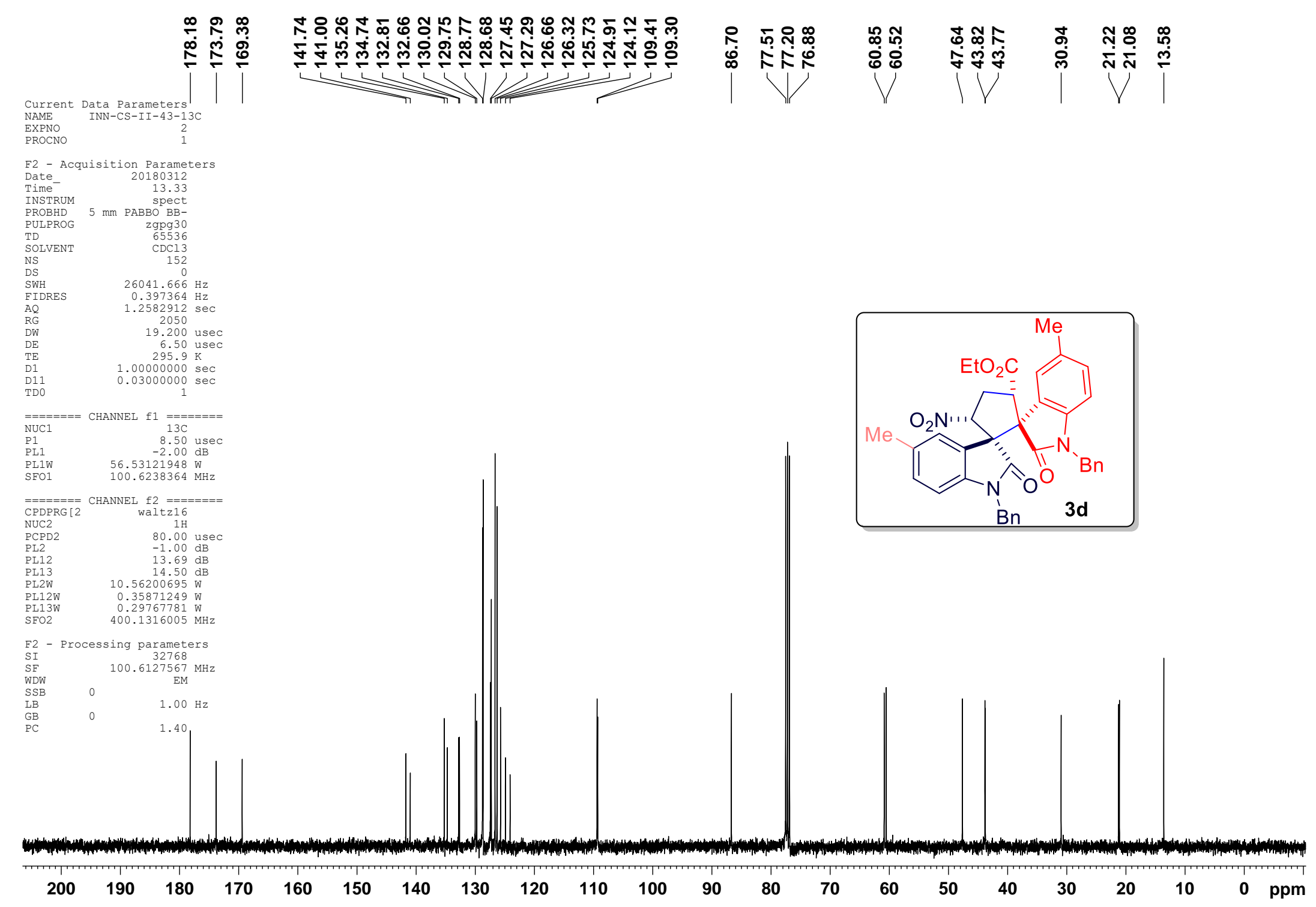

Figure S30. ${ }^{13} \mathrm{C}$ NMR $\left(100 \mathrm{MHz}, \mathrm{CDCl}_{3}\right)$ Spectrum of $\mathbf{3 d}$ 

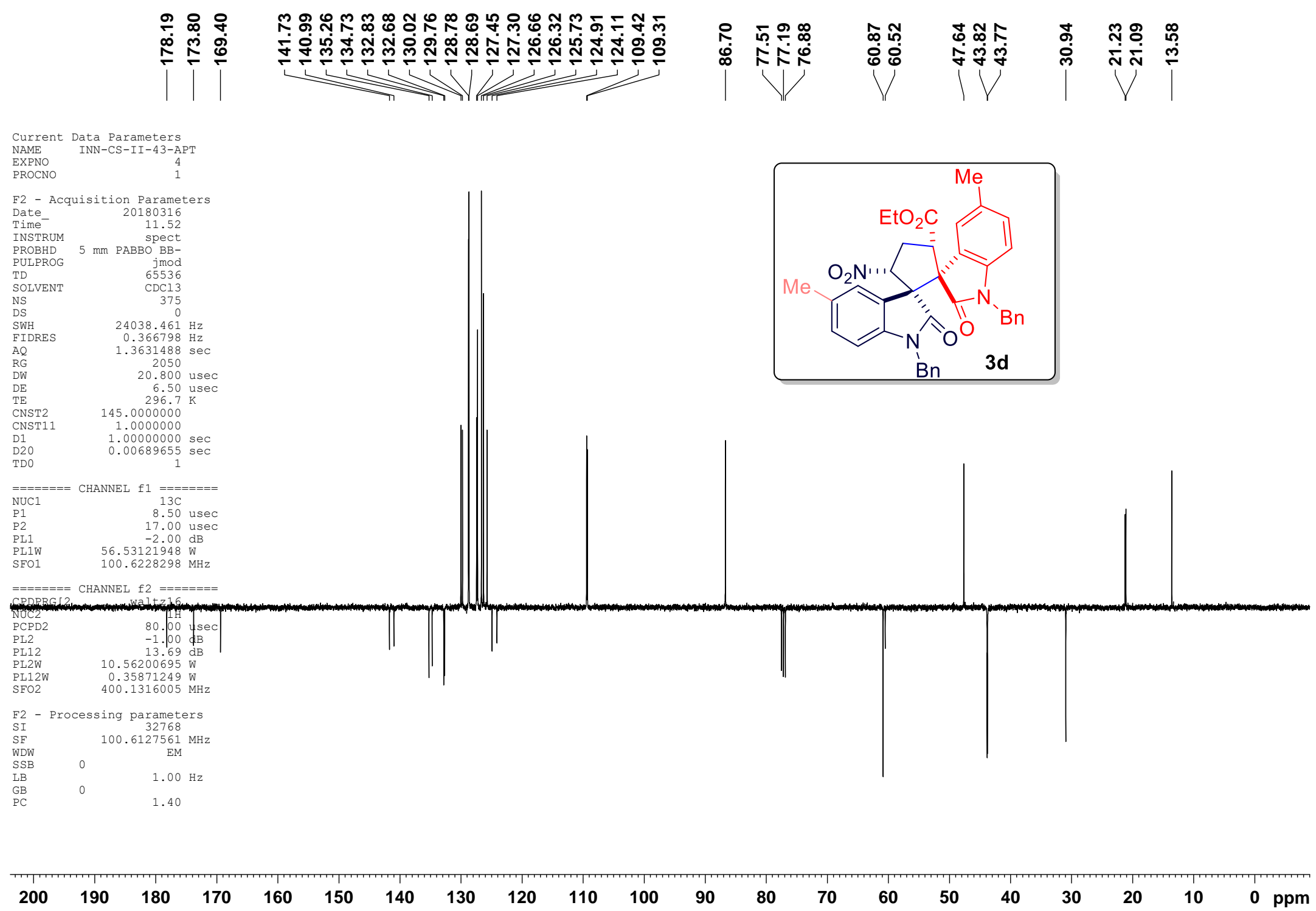

Figure S31. ${ }^{13} \mathrm{C}$-APT NMR (100 MHz, $\left.\mathrm{CDCl}_{3}\right)$ Spectrum of 3d 
INN-CS-II-43-COSY

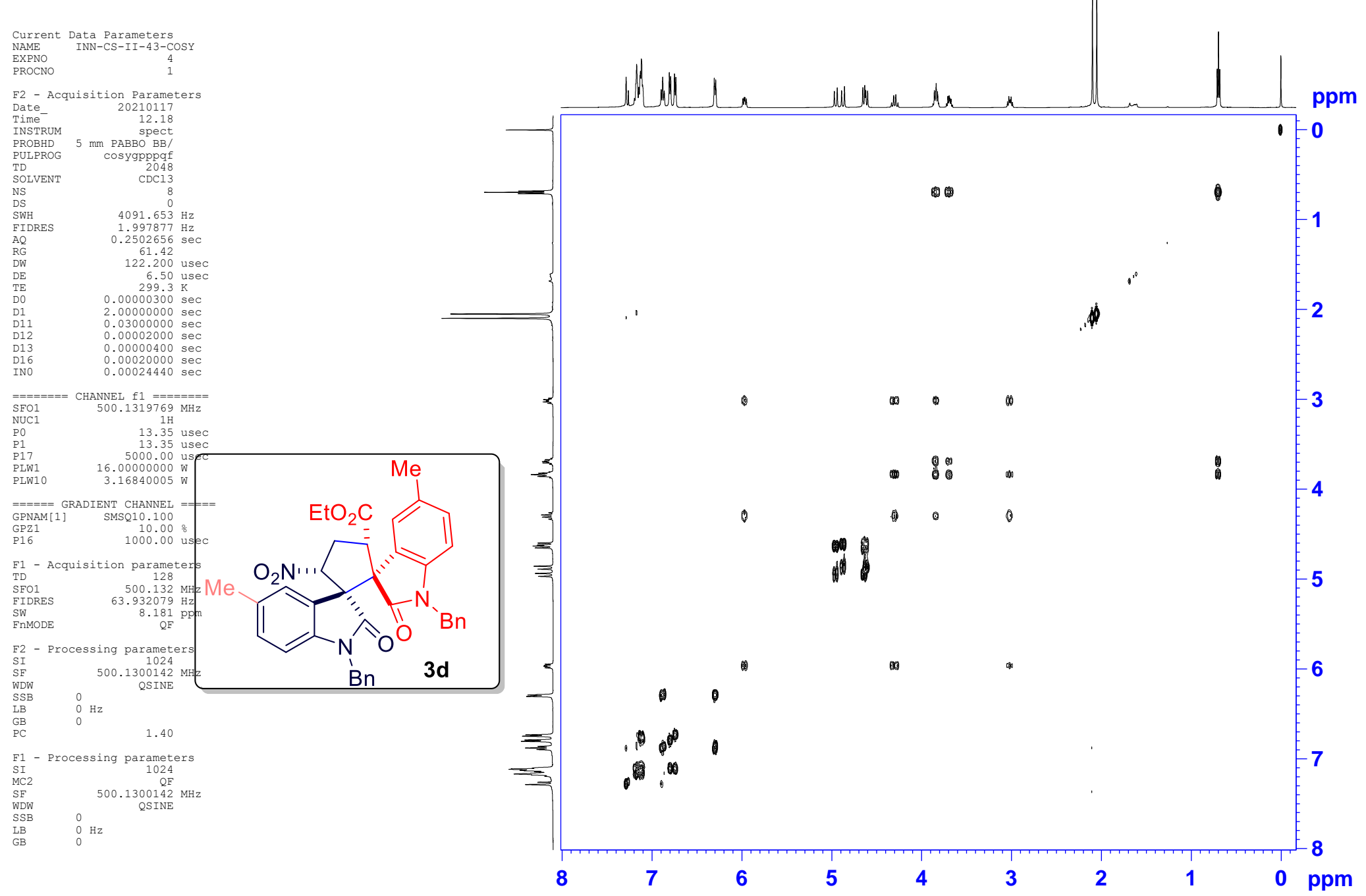

Figure S32. ${ }^{1} \mathrm{H}-\mathrm{H}$ COSY NMR $\left(500 \mathrm{MHz}, \mathrm{CDCl}_{3}\right)$ Spectrum of 3d 

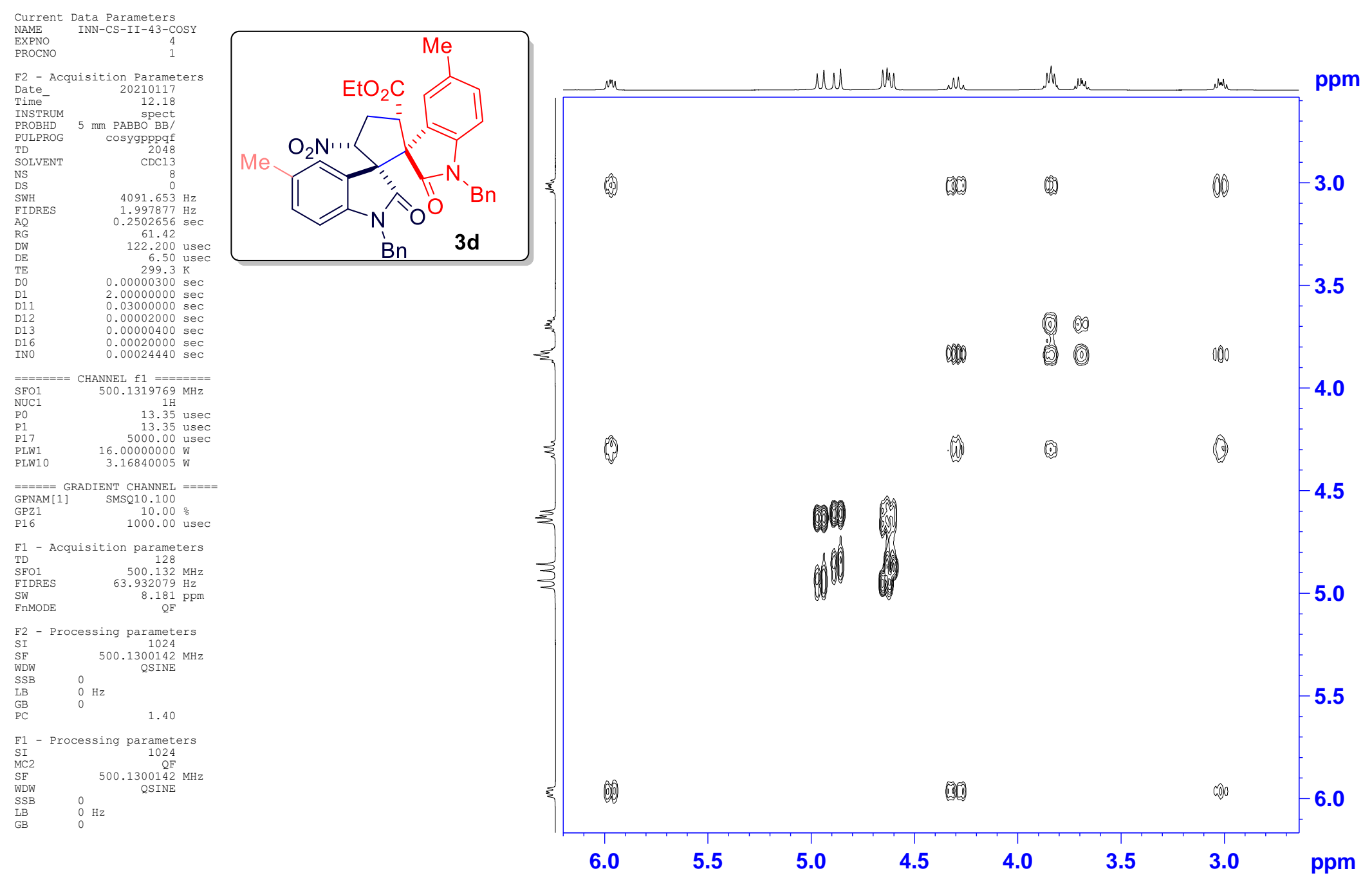

Figure S33. ${ }^{1} \mathrm{H}-\mathrm{H}$ COSY NMR (500 MHz, $\mathrm{CDCl}_{3}$ ) Spectrum (Expansion) of 3d 


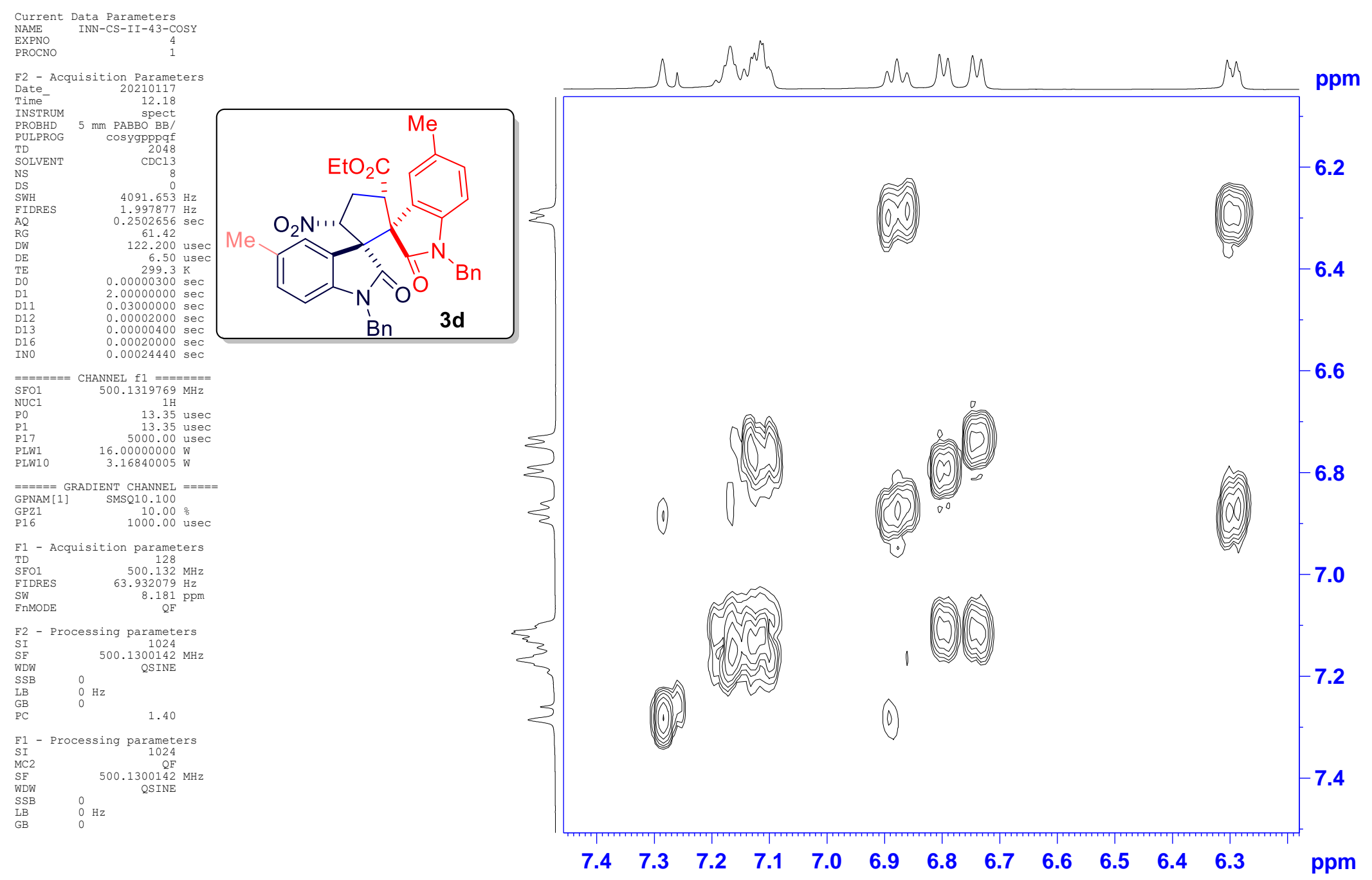

Figure S34. ${ }^{1} \mathrm{H}-\mathrm{H}$ COSY NMR (500 MHz, $\mathrm{CDCl}_{3}$ ) Spectrum (Expansion) of 3d 


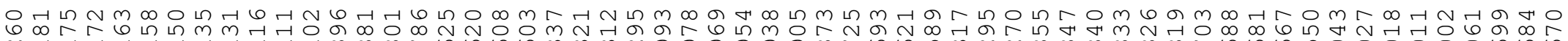

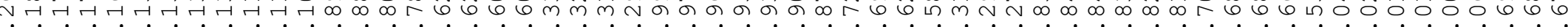

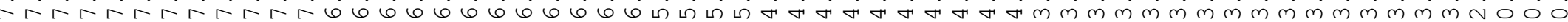

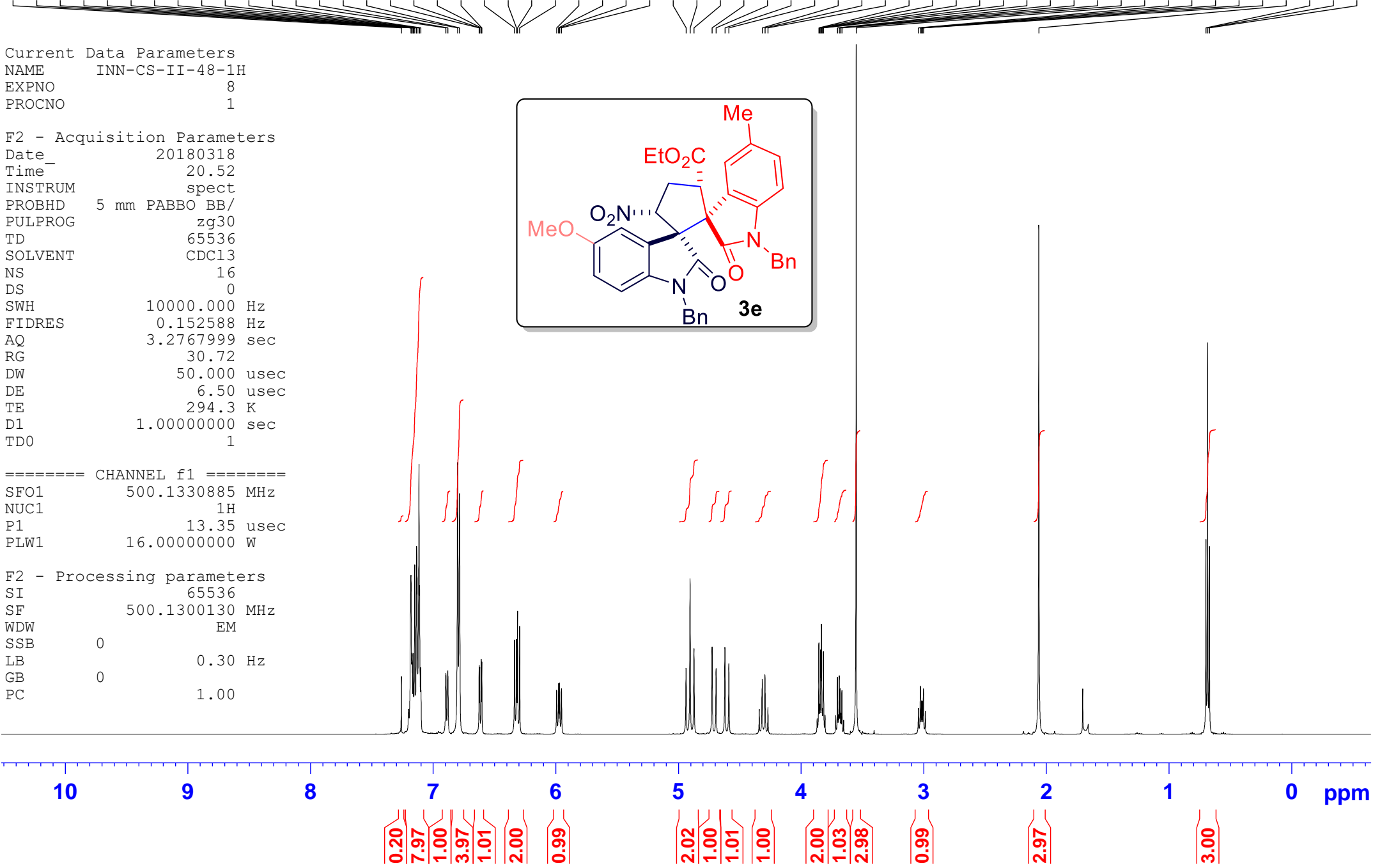

Figure S35. ${ }^{1} \mathrm{H} \mathrm{NMR}\left(500 \mathrm{MHz}, \mathrm{CDCl}_{3}\right)$ Spectrum of $\mathbf{3 e}$ 


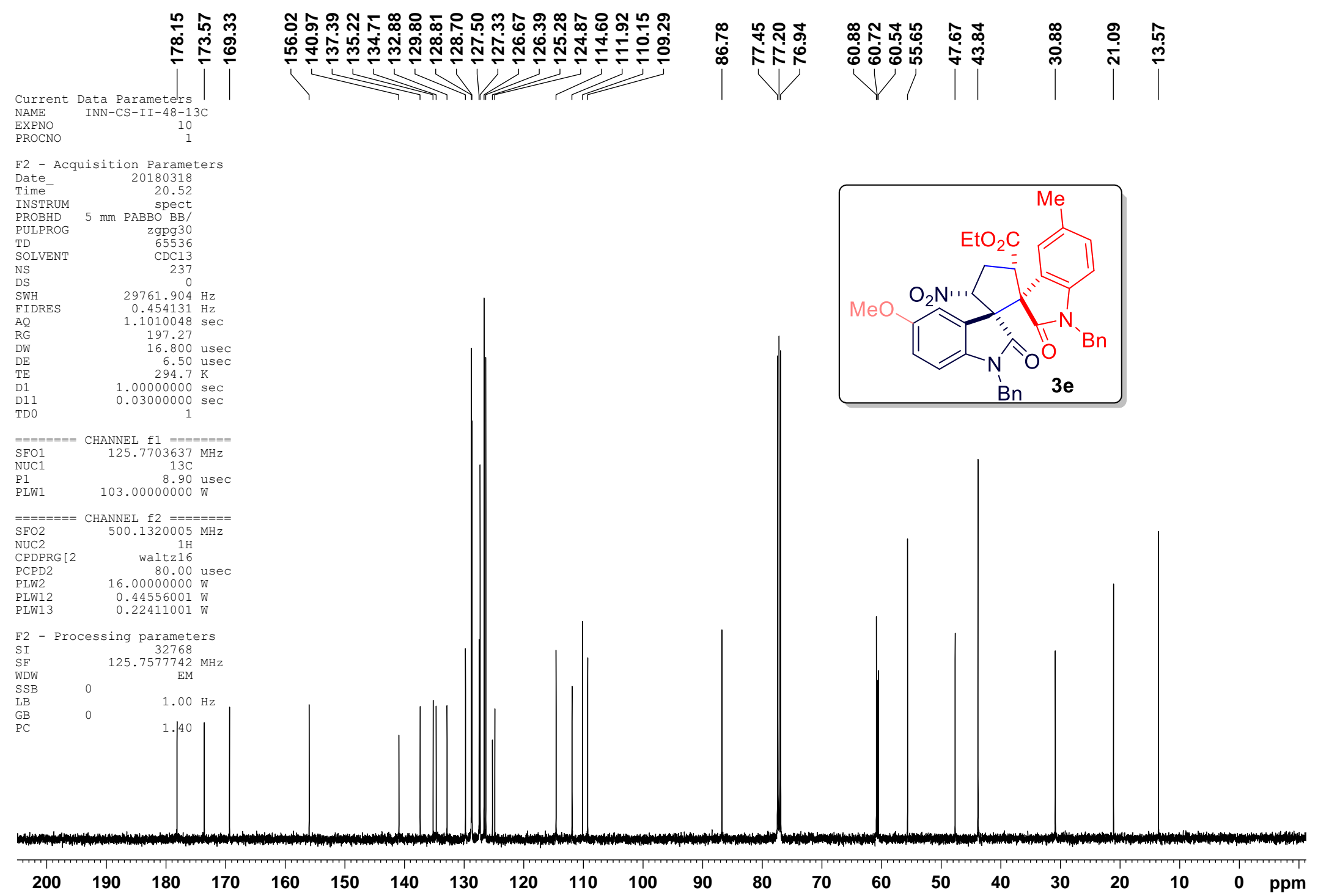

Figure S36. ${ }^{13} \mathrm{C}$ NMR $\left(125 \mathrm{MHz}, \mathrm{CDCl}_{3}\right)$ Spectrum of $\mathbf{3 e}$ 


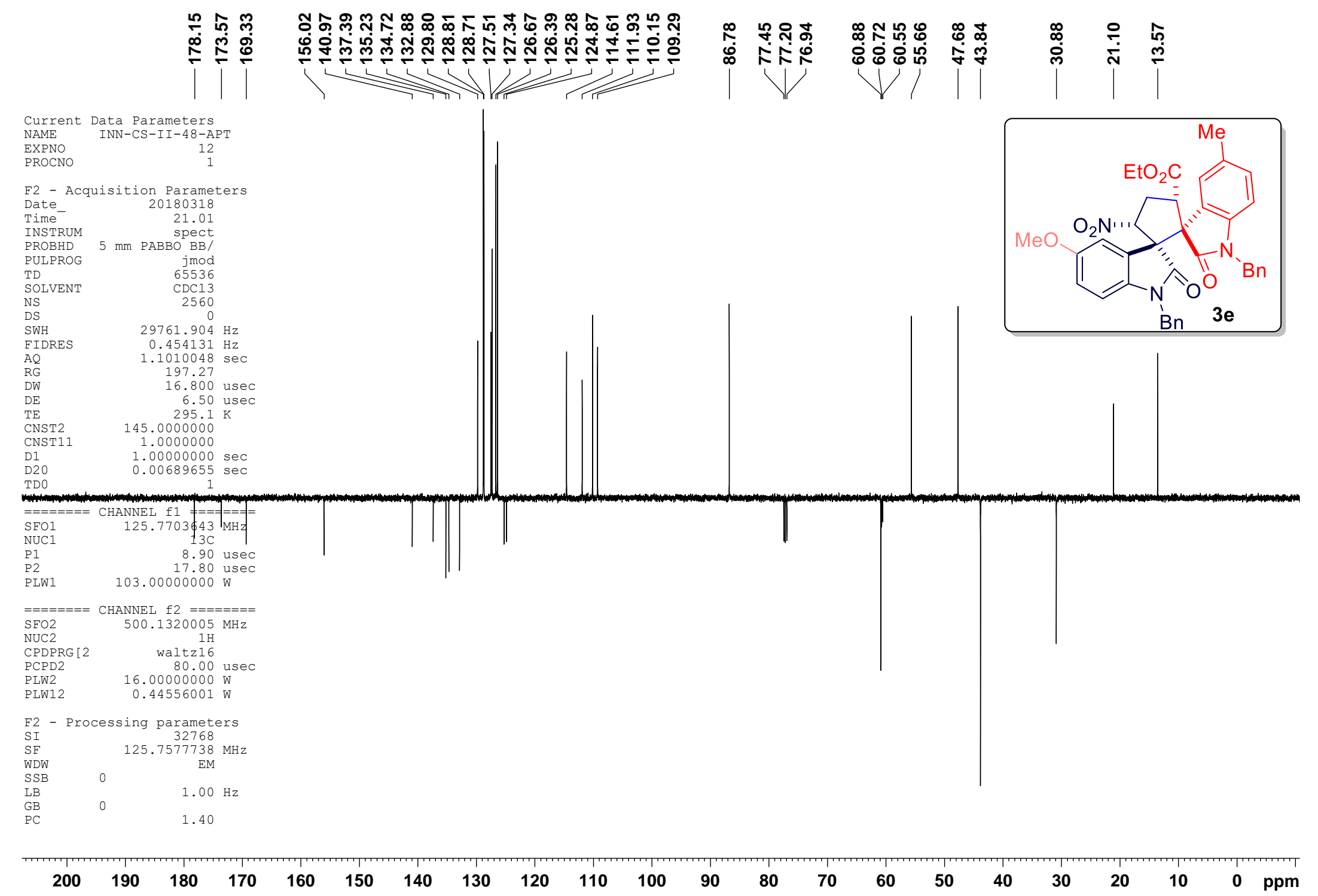

Figure S37. ${ }^{13} \mathrm{C}-\mathrm{APT}$ NMR (125 MHz, $\mathrm{CDCl}_{3}$ ) Spectrum of $\mathbf{3 e}$ 


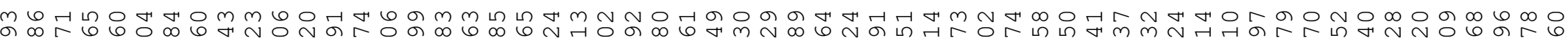

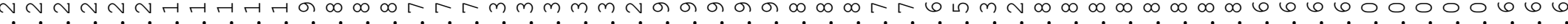

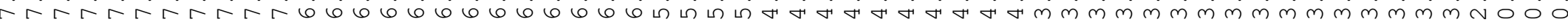
$\begin{array}{lc}\text { Current } & \text { Data Parameters } \\ \text { NAME } & \text { INN-CS-II-50-1H } \\ \text { EXPNO } & 1 \\ \text { PROCNO } & 1\end{array}$

F2 - Acquisition Parameters

$\begin{array}{rr}\text { Date } & 20180402 \\ \text { Time } & 14.25\end{array}$

INSTRUM SPect

$5 \mathrm{~mm}$ PABBO BB-

TD

SOLVENT

NS

$\begin{array}{lr}\text { DS } & 0 \\ \text { SWH } & 8223.685 \mathrm{~Hz}\end{array}$

FIDRES $\quad 0.151522 \mathrm{~Hz}$

AQ $\quad 3.2998593 \mathrm{sec}$

DW $\quad 60.800$ usec

DE $\quad 6.50$ usec

D1 $1.00000000 \mathrm{sec}$

TDO

$=======$ CHANNEL $\mathrm{f} 1$

1

$\begin{array}{lr}\text { NUC1 } & 1 \mathrm{H} \\ \mathrm{P} 1 & 14.75 \\ \mathrm{P} & -1.00 \mathrm{usec}\end{array}$

$\begin{array}{lr}\text { PL1 } & -1.00 \mathrm{~dB} \\ \text { PL1W } & 10.56200695 \mathrm{~W}\end{array}$

SFO1 $400.1324710 \mathrm{MHz}$

F2 - Processing parameters

SI 32768

$\begin{array}{ll}\mathrm{SF} & 400.1300102 \mathrm{MHz}\end{array}$

$\begin{array}{ll}\text { WDW } & 0 \\ \text { LSB } & 0.30 \mathrm{~Hz}\end{array}$

$\begin{array}{lll}\mathrm{LB} & 0 & 0.30 \\ \mathrm{~GB} & 0 & 1.00\end{array}$

PC 1.00

10

8
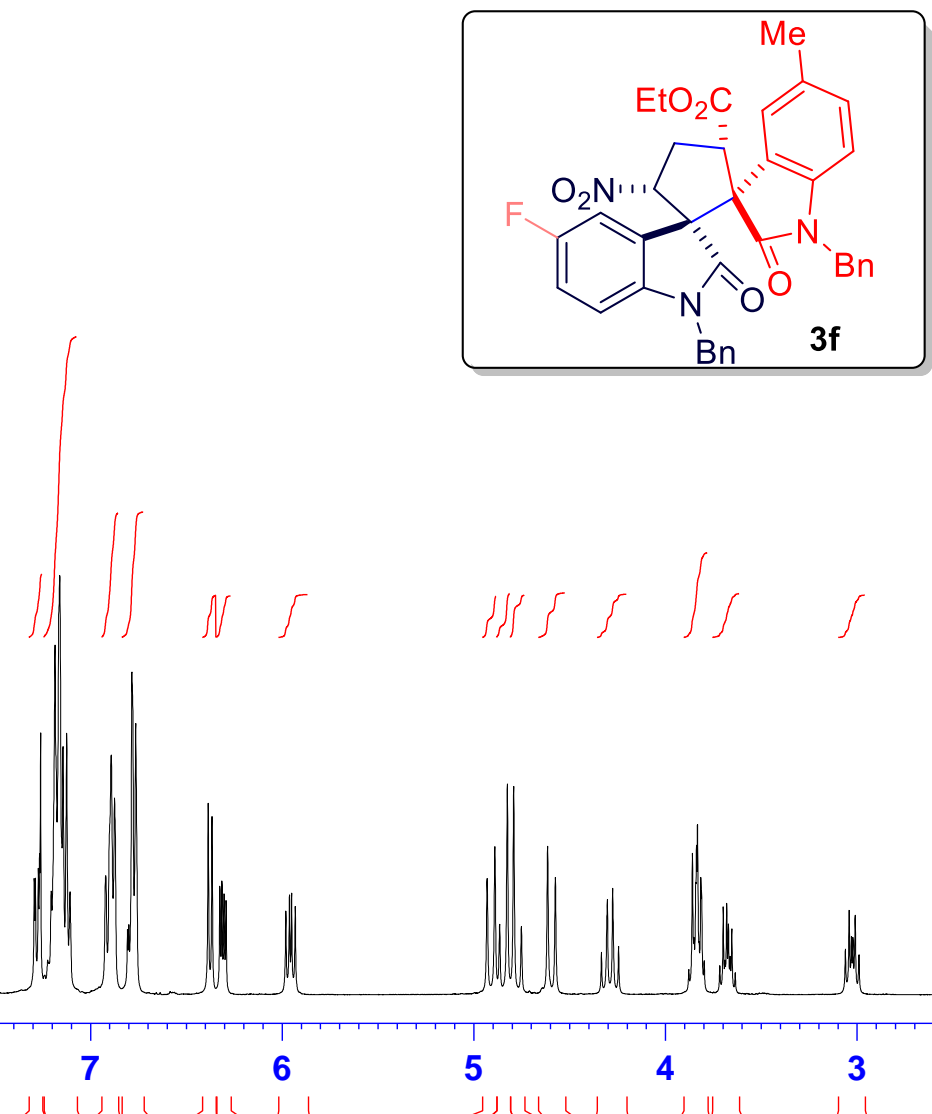

7

6

卢|

5

4

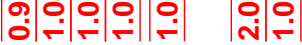

Figure S38. ${ }^{1} \mathrm{H}$ NMR (400 $\mathrm{MHz}, \mathrm{CDCl}_{3}$ ) Spectrum of $\mathbf{3 f}$ 


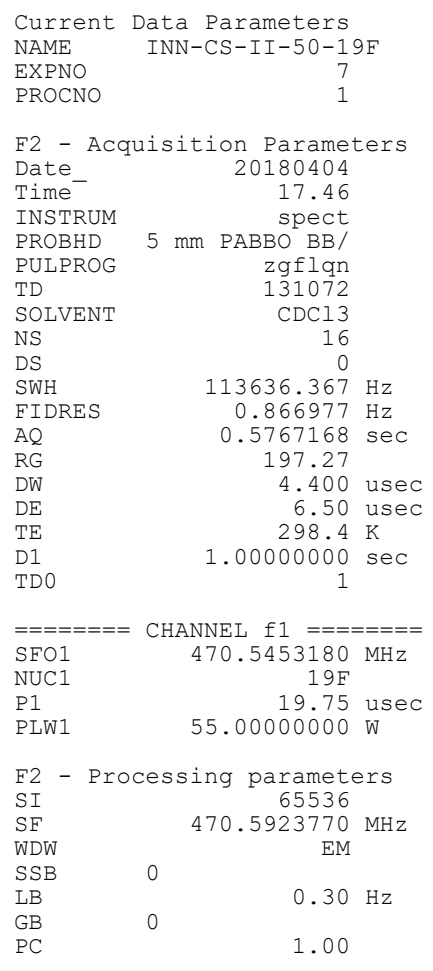

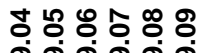 \\ 웅ำ웅 웅}

$\div \div \div \div$

4

Date Acquisition Parameter

DS $0.866977 \mathrm{~Hz}$ $5767168 \mathrm{sec}$
197.27

4.400 usec

$298.4 \mathrm{~K}$

NEL f $1======$

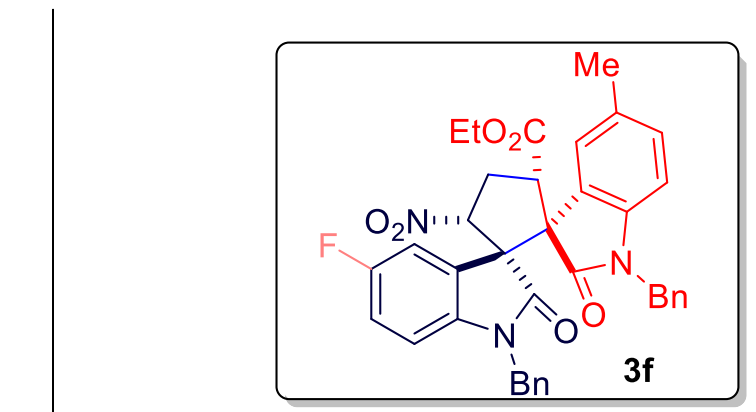

SE $\quad 470.5923770 \mathrm{MHz}$

$\underset{\mathrm{LB}}{\mathrm{SSB}} \quad 0$

$\begin{array}{ll}\mathrm{GB} & 1.00\end{array}$

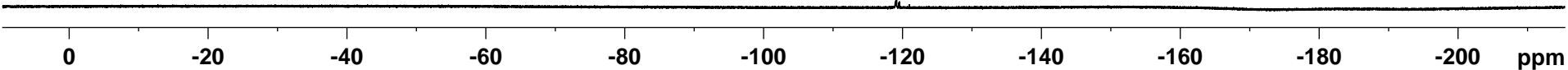

Figure S39. ${ }^{19} \mathrm{~F}$ NMR (470 $\left.\mathrm{MHz}, \mathrm{CDCl}_{3}\right)$ Spectrum of $\mathbf{3 f}$ 


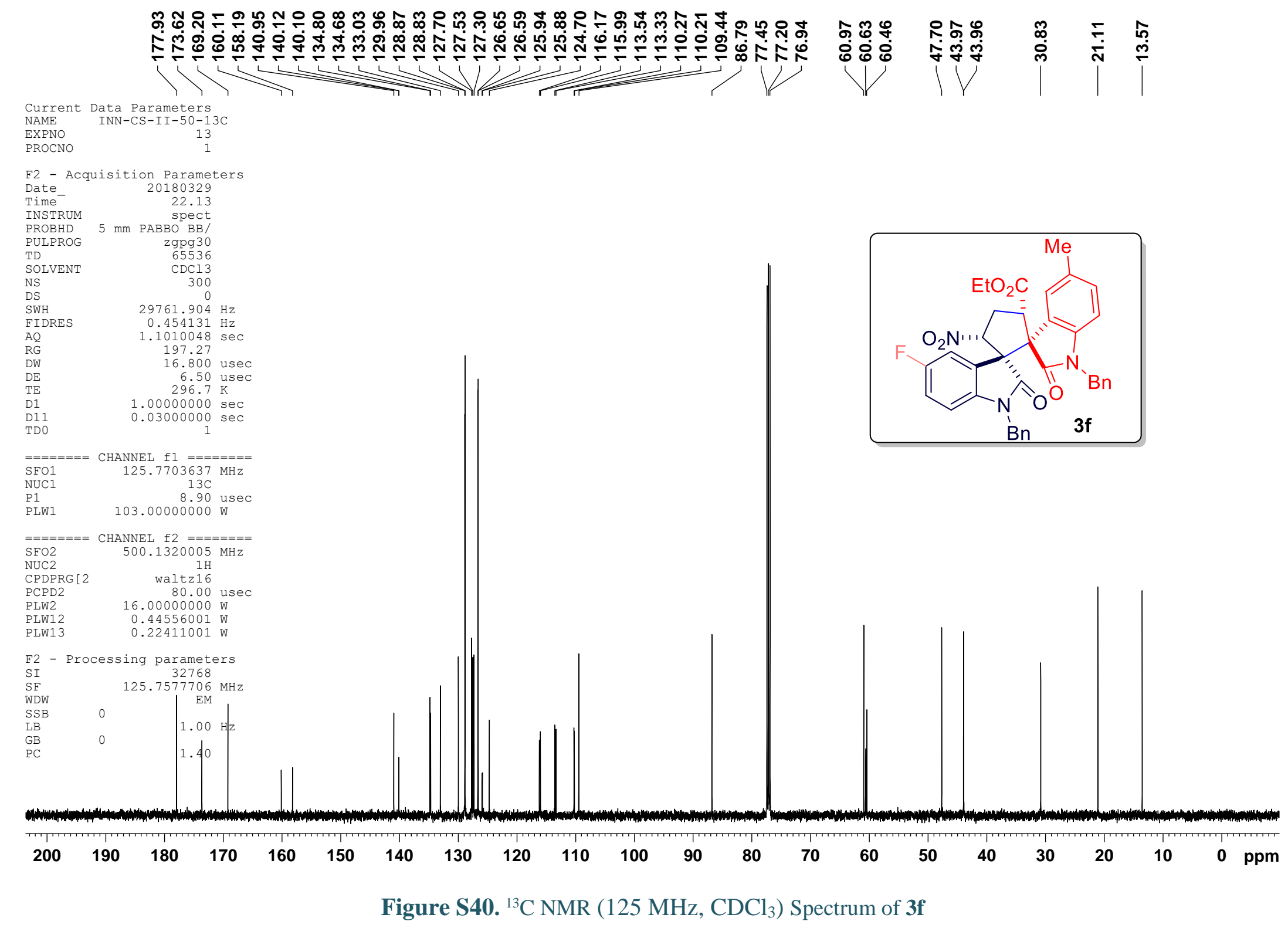




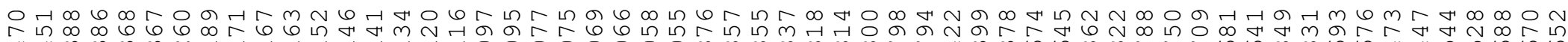
T. T.

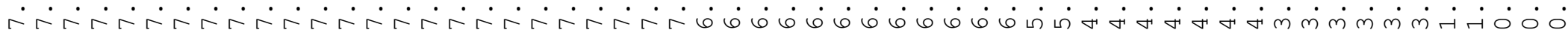

(1)

Current Data Parameters

NAME INN-CS-II-39-1H

EXPNO

PROCNO

F2 - Acquisition Parameters

Date_ 20180307

Time 15.50

PROBHD $5 \mathrm{~mm}$ PABBO BB-

PULPROG 5930

TD 54274

SOLVEN'

NS

$\begin{array}{ll}8223.685 \mathrm{~Hz} \\ \text { SWH } & 0.151522 \mathrm{~Hz}\end{array}$

IDRES $\quad 0.151522 \mathrm{~Hz}$

AQ

$3.2998593 \mathrm{sec}$

128
60.800 use

60.800 usec

$\begin{array}{lr}\mathrm{DE} & 6.50 \mathrm{use} \\ \mathrm{TE} & 296.5 \mathrm{~K}\end{array}$

$\begin{array}{lr}\text { D1 } & 1.00000000 \mathrm{sec} \\ \text { TD } & 1\end{array}$

$========$ CHANNEL f 1 l $========$

$\mathrm{NUCl}$

P1 1

PL1

4.75 usec

$-1.00 \mathrm{~dB}$

$\begin{array}{ll} & 10.56200695 \mathrm{~W} \\ \text { SFO1 } & 400.1324710 \mathrm{MHz}\end{array}$

F2 - Processing parameters

SI 32768

SF $\quad 400.1300101 \mathrm{MHz}$

$\begin{array}{lll}\text { WDW } & & \text { EM } \\ \text { SSB } & 0\end{array}$

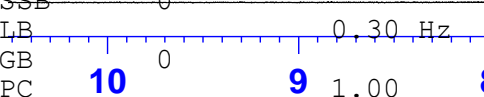

8

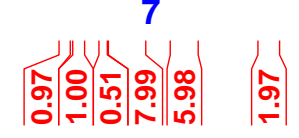

$(\bar{c})$

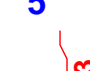

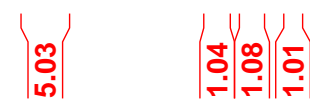

3

Figure S41. ${ }^{1} \mathrm{H}$ NMR (400 $\mathrm{MHz}, \mathrm{CDCl}_{3}$ ) Spectrum of $\mathbf{3 g}$ 


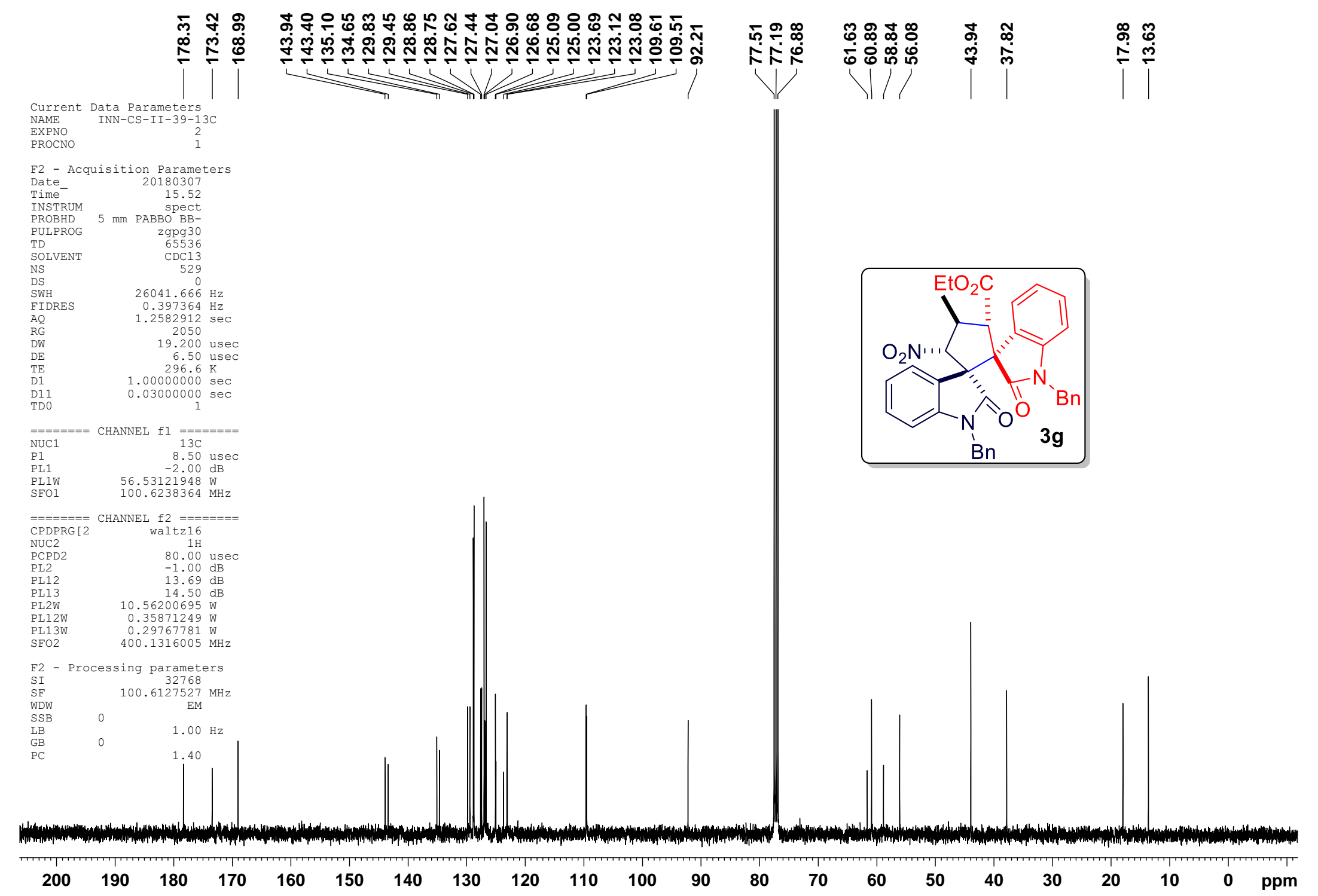

Figure S42. ${ }^{13} \mathrm{C}$ NMR $\left(100 \mathrm{MHz}, \mathrm{CDCl}_{3}\right)$ Spectrum of $\mathbf{3 g}$ 


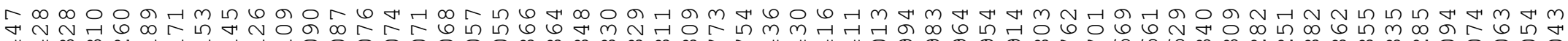

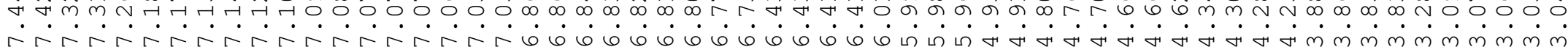

Current Data Parameters

NAME INN-CS-II-37-1H

EXPNO

1

F2 - Acquisition Parameters

Date_ 20180217

Time 11.44

$5 \mathrm{~mm}$ PABBOCt

PULPRO 5 mim PABBO
zg30

TD $\quad 54274$

SOLVENT

NS

SWH

8223.685 Hz

$0.151522 \mathrm{~Hz}$

$\begin{array}{lr}\text { AQ } & 3.2998593 \\ \text { RG } & 90.5\end{array}$

$\begin{array}{lr}\text { RG } & 90.5 \\ \text { DW } & 60.800 \text { usec }\end{array}$

DE $\quad 6.50$ usec

$296.8 \mathrm{~K}$
$\mathrm{TE}$

TDO

$=======$ CHANNEL

NUC1

PL1 1

PL1W
SFO1

F2 - Processing parameters

FI - Processing parameters

SF $\quad 400.1300100 \mathrm{MHz}$

WDW

$\mathrm{SSB}$

$\mathrm{EM}$

$1========$

75 use

$.00 \mathrm{~dB}$

$\begin{array}{lll}\mathrm{LB} & 0 & 0.30 \mathrm{~Hz}\end{array}$

10
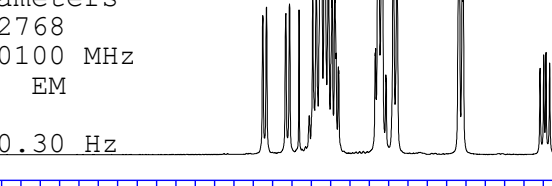

6

4

श्:

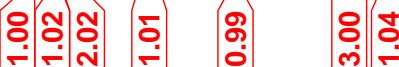

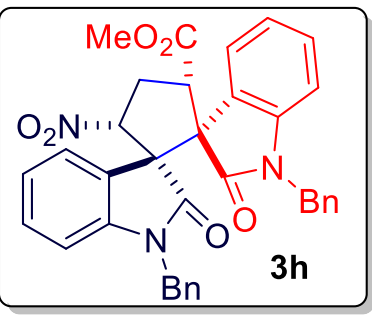

Figure S43. ${ }^{1} \mathrm{H}$ NMR $\left(400 \mathrm{MHz}, \mathrm{CDCl}_{3}\right)$ Spectrum of $\mathbf{3 h}$ 


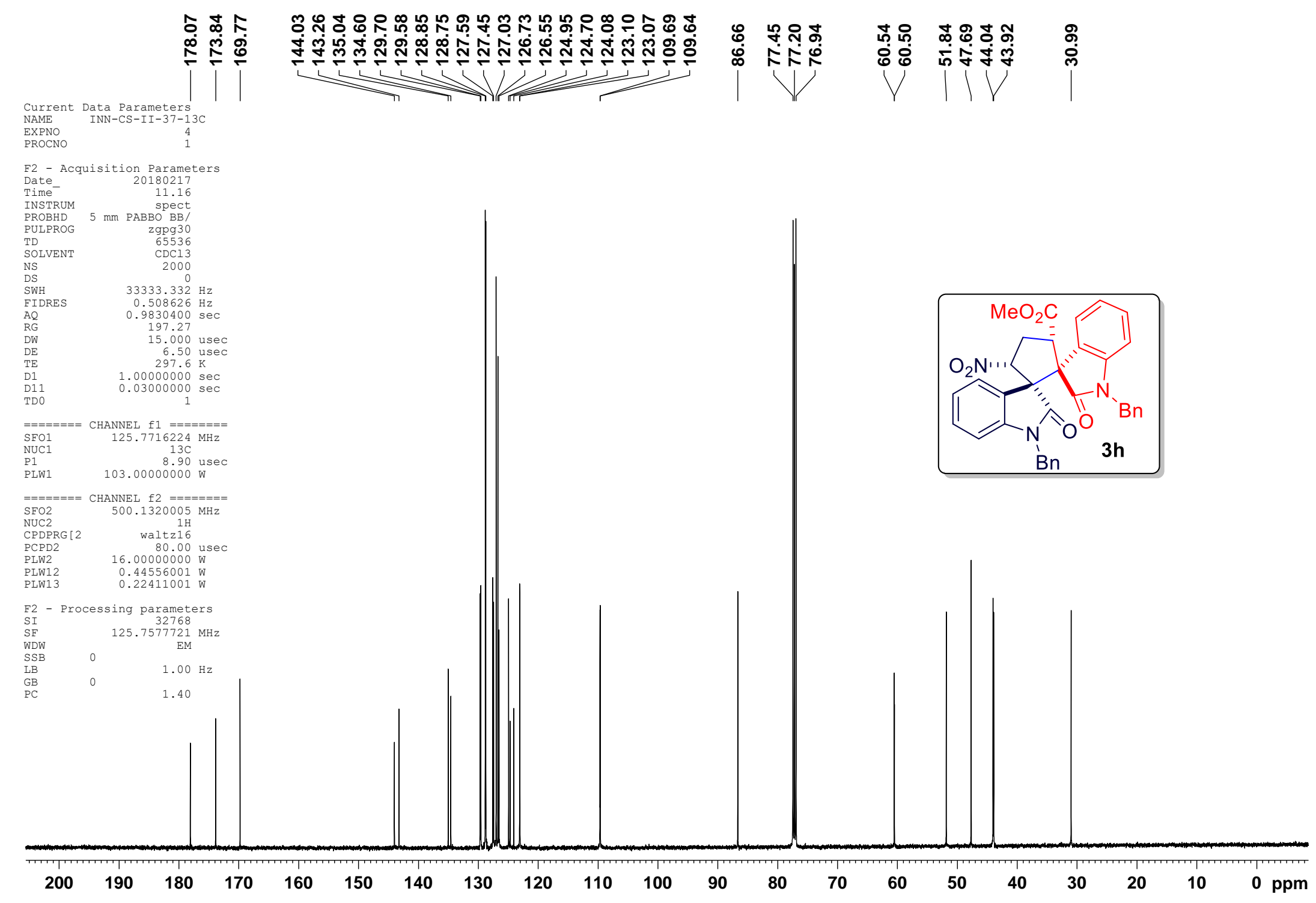

Figure S44. ${ }^{13} \mathrm{C}$ NMR $\left(125 \mathrm{MHz}, \mathrm{CDCl}_{3}\right)$ Spectrum of $\mathbf{3 h}$ 
OกMN -

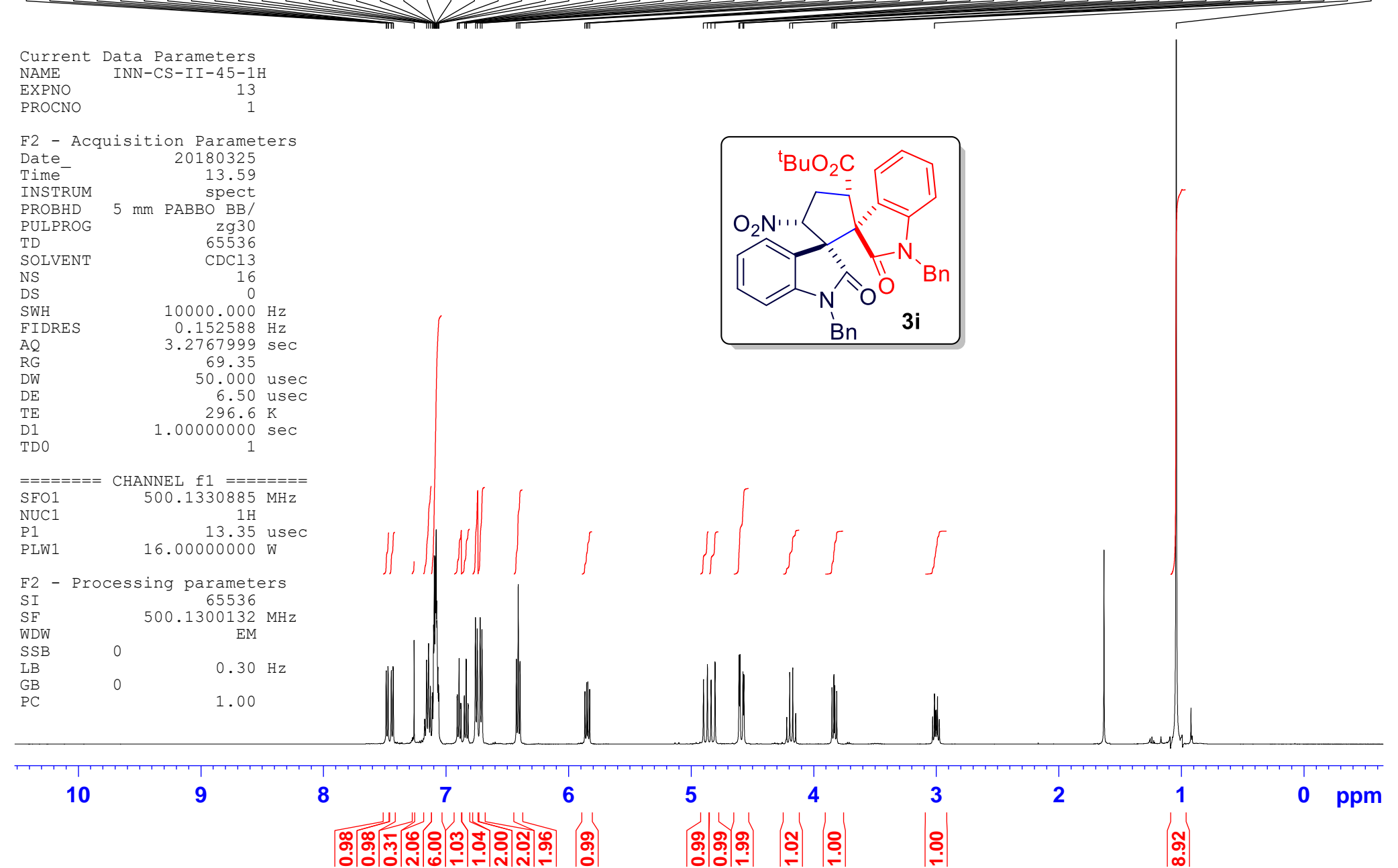

Figure S45. ${ }^{1} \mathrm{H}$ NMR $\left(500 \mathrm{MHz}, \mathrm{CDCl}_{3}\right)$ Spectrum of $\mathbf{3 i}$ 


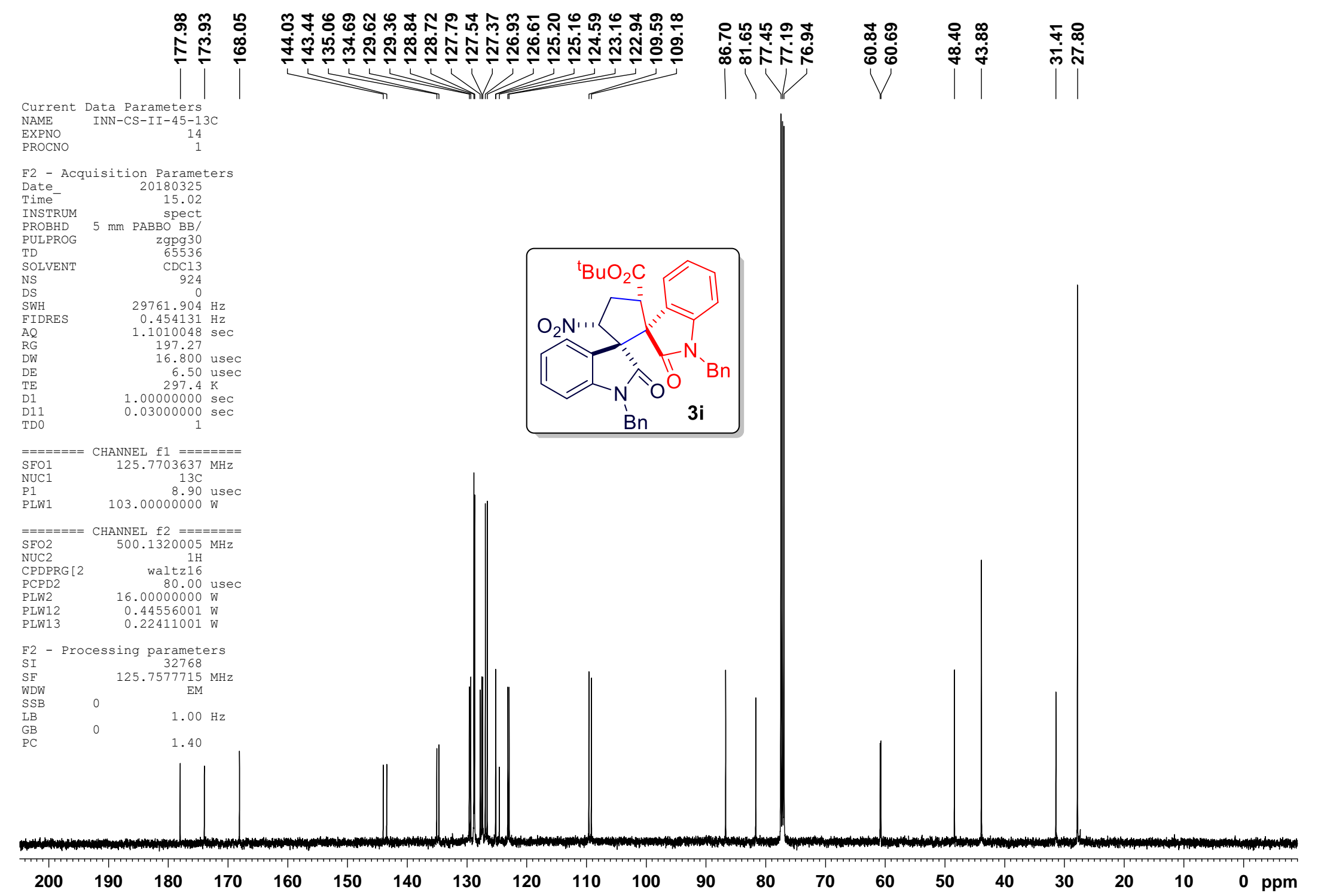

Figure S46. ${ }^{13} \mathrm{C}$ NMR $\left(125 \mathrm{MHz}, \mathrm{CDCl}_{3}\right)$ Spectrum of $\mathbf{3 i}$ 
GRO orman

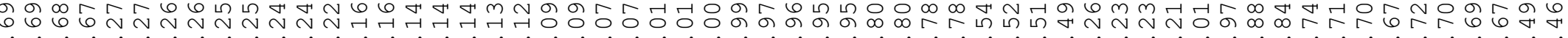

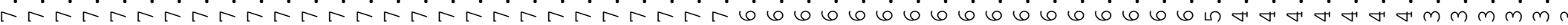

Current Data Parameters

NAME INN-CS-II-21-1H

PROCNO

F2 - Acquisition Parameters

Date__ 20171204

INSTRUM

spect
PROBHD $5 \mathrm{~mm}$ PABBO BB-

PULPROG

TD 54274

SOLVENT

NS
DS

SWH

FIDRES

AQ

RG

DW

DE

D1

$=======$ CHANNEL $f$

NUC1

$\begin{array}{ll} & 10.56200695 \mathrm{~W} \\ \text { SFO1 } & 400.1324710 \mathrm{MHz}\end{array}$

F2 - Processing parameters

SI 32768

$400.1300095 \mathrm{MH}$

$\begin{array}{lll}\mathrm{SSB} & 0 & 0.30 \mathrm{~Hz}\end{array}$

10

9

$8223.685 \mathrm{~Hz}$

$0.151522 \mathrm{~Hz}$

144

0.800 usec

$296.9 \mathrm{~K}$

$\pi \pi \pi$

m 11

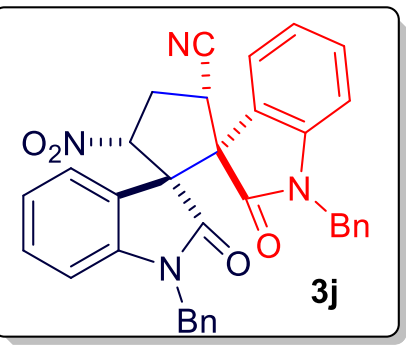

ol

|ำ

6

(a)

(2)

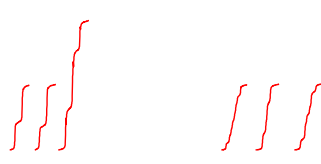

Figure S47. ${ }^{1} \mathrm{H}$ NMR (400 $\left.\mathrm{MHz}, \mathrm{CDCl}_{3}\right)$ Spectrum of $\mathbf{3 j}$ 


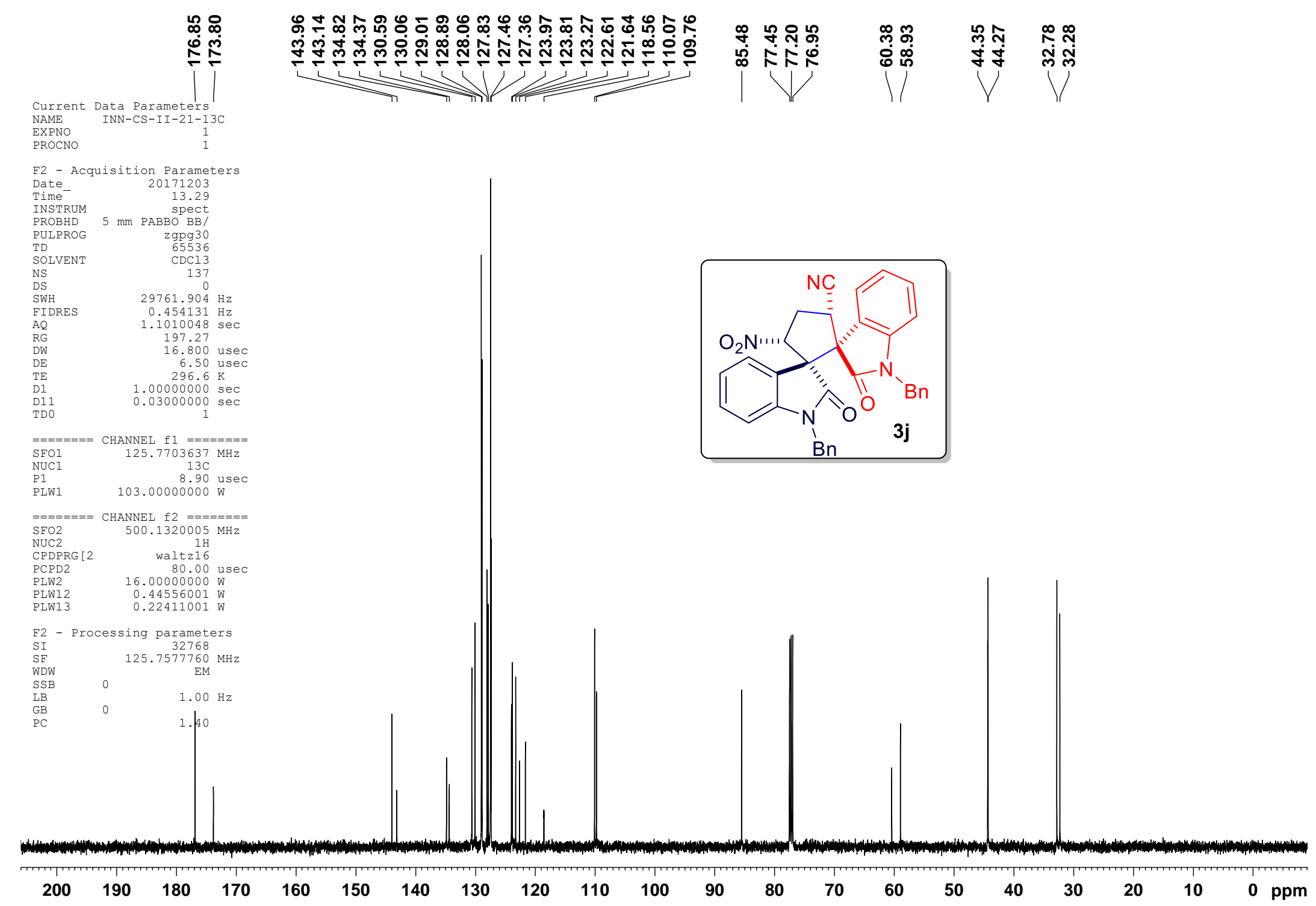

Figure S48. ${ }^{13} \mathrm{C}$ NMR $\left(125 \mathrm{MHz}, \mathrm{CDCl}_{3}\right)$ Spectrum of $\mathbf{3 j}$ 


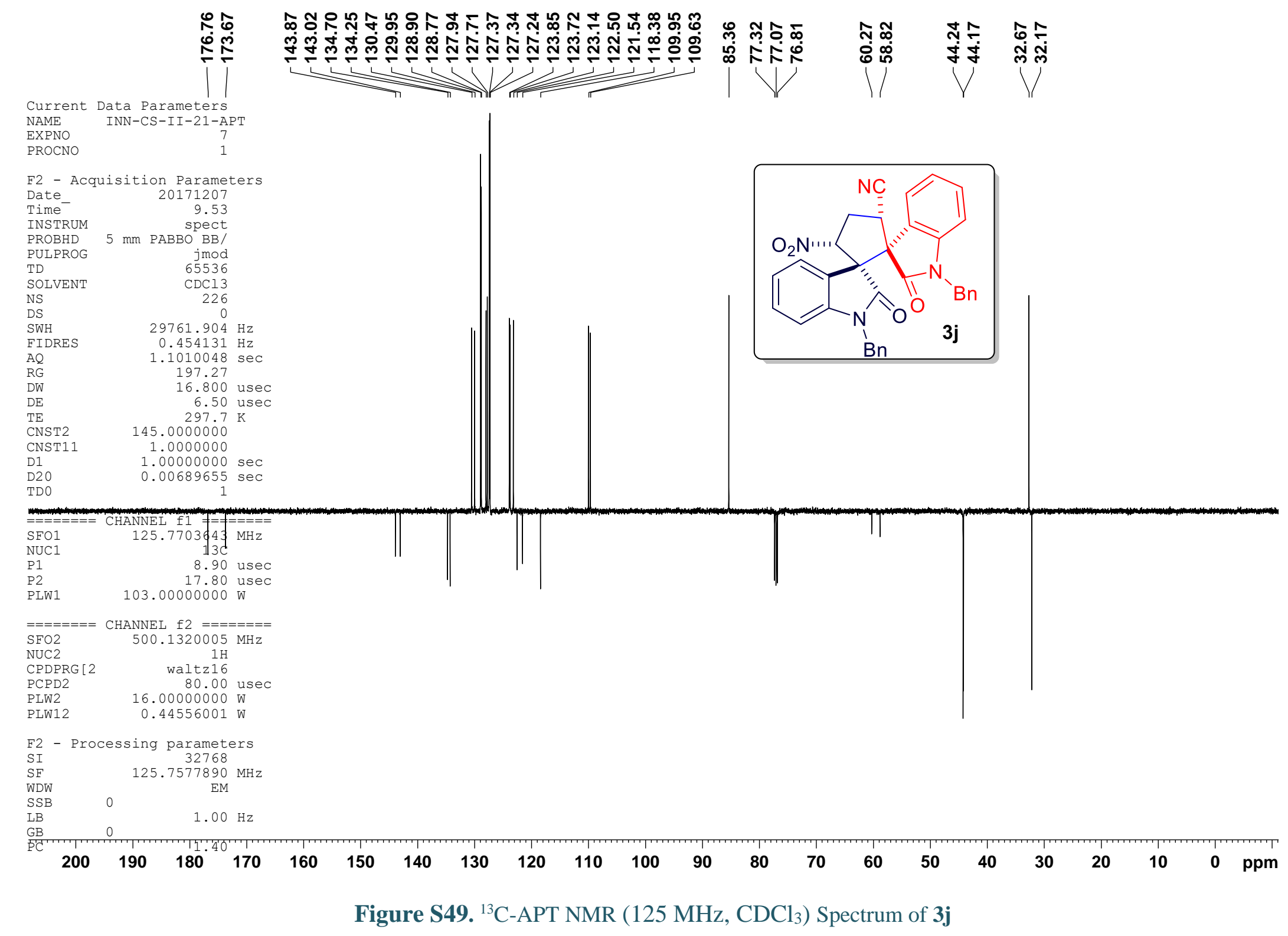


INN-CS-II-21-COSY

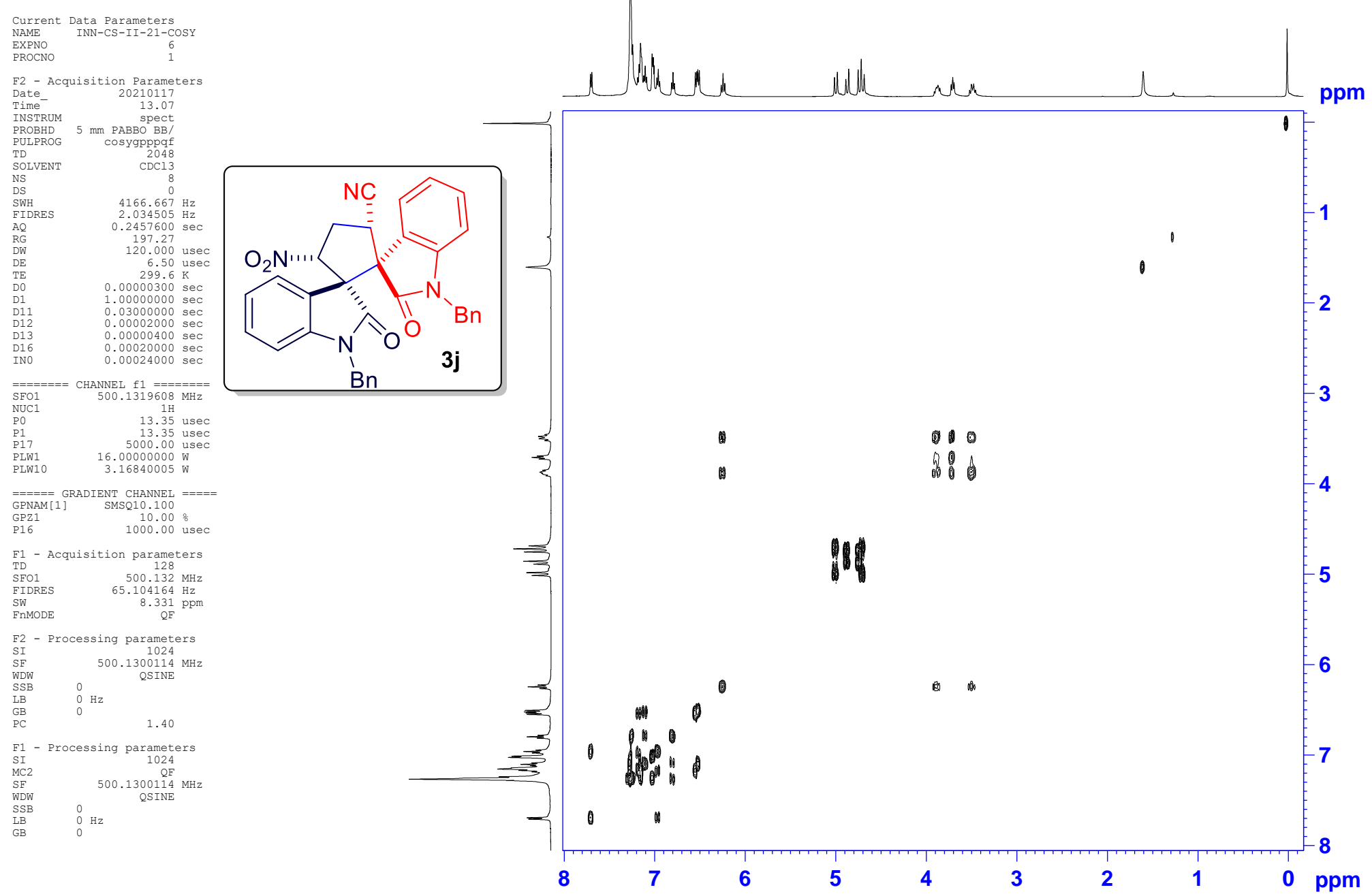

Figure S50. ${ }^{1} \mathrm{H}-\mathrm{H}$ COSY NMR $\left(500 \mathrm{MHz}, \mathrm{CDCl}_{3}\right)$ Spectrum of $\mathbf{3 j}$ 
INN-CS-II-21-COSY

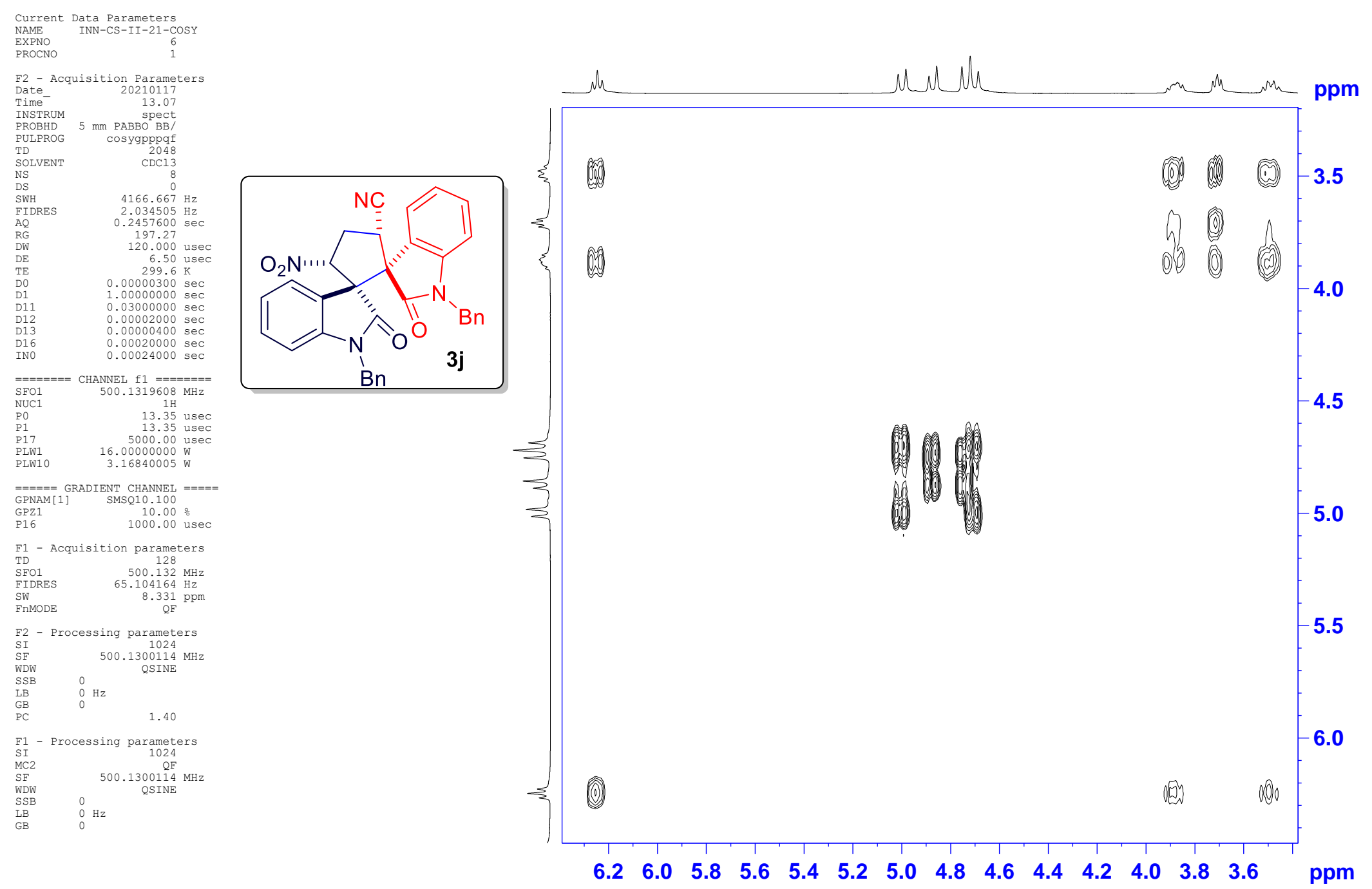

Figure S51. ${ }^{1} \mathrm{H}-\mathrm{H}$ COSY NMR (500 MHz, $\left.\mathrm{CDCl}_{3}\right)$ Spectrum (Expansion) of $\mathbf{3 j}$ 
INN-CS-II-21-COSY
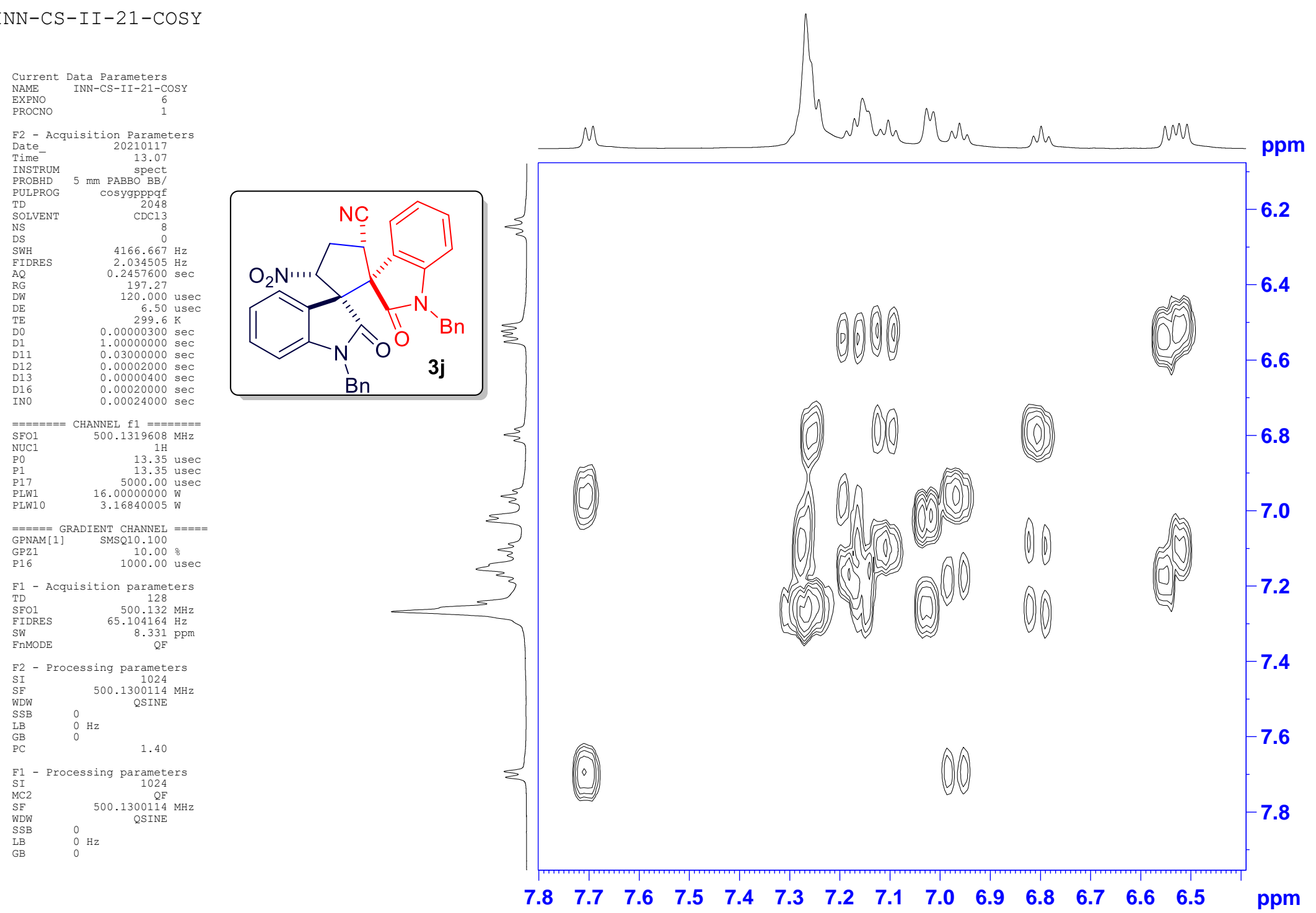

Figure S52. ${ }^{1} \mathrm{H}-\mathrm{H}$ COSY NMR (500 MHz, $\left.\mathrm{CDCl}_{3}\right)$ Spectrum (Expansion) of $\mathbf{3 j}$ 


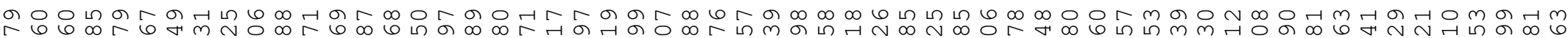

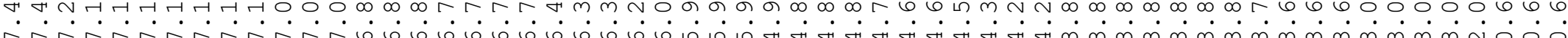

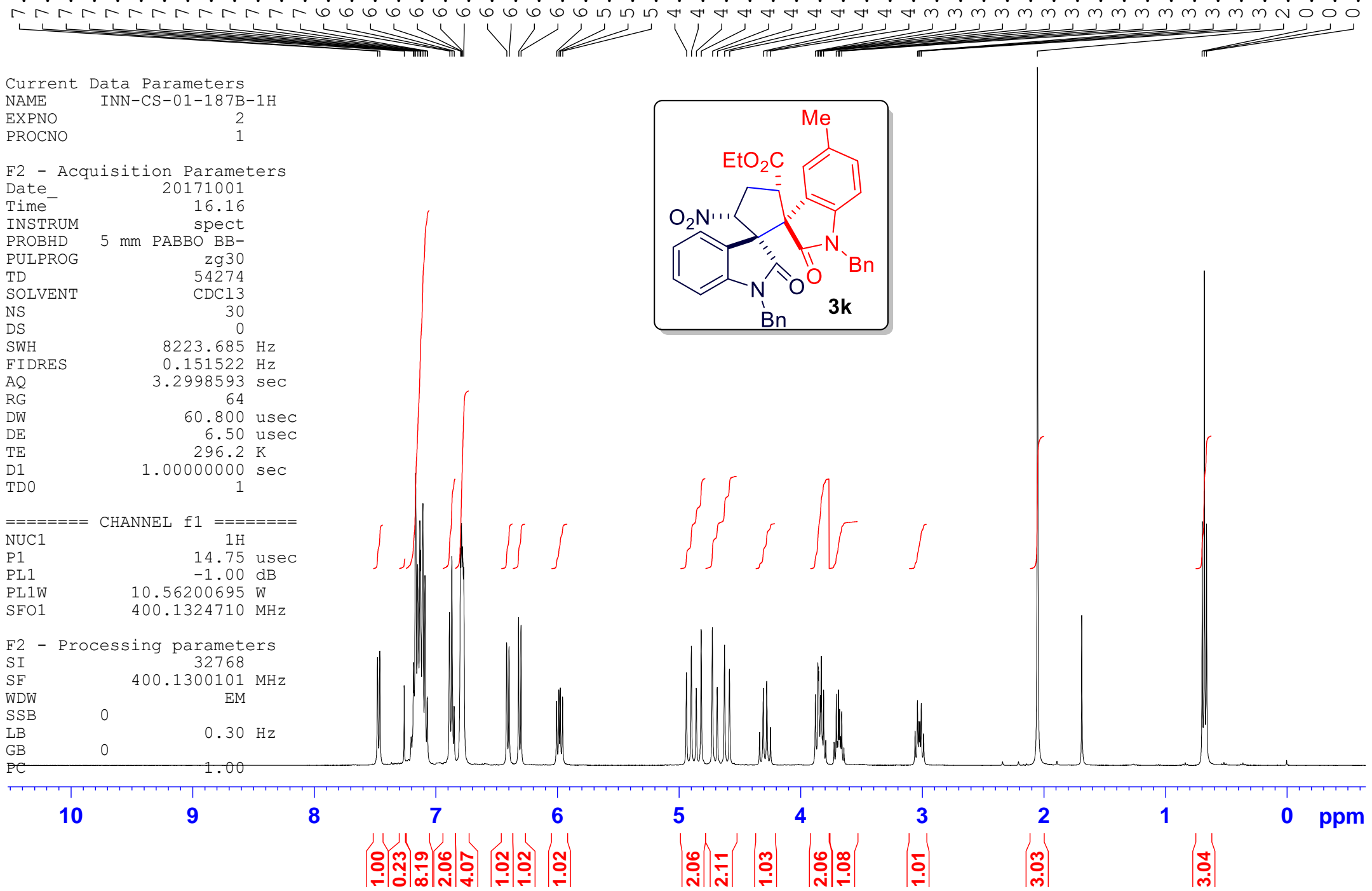

Figure S53. ${ }^{1} \mathrm{H}$ NMR $\left(400 \mathrm{MHz}, \mathrm{CDCl}_{3}\right)$ Spectrum of $\mathbf{3 k}$ 


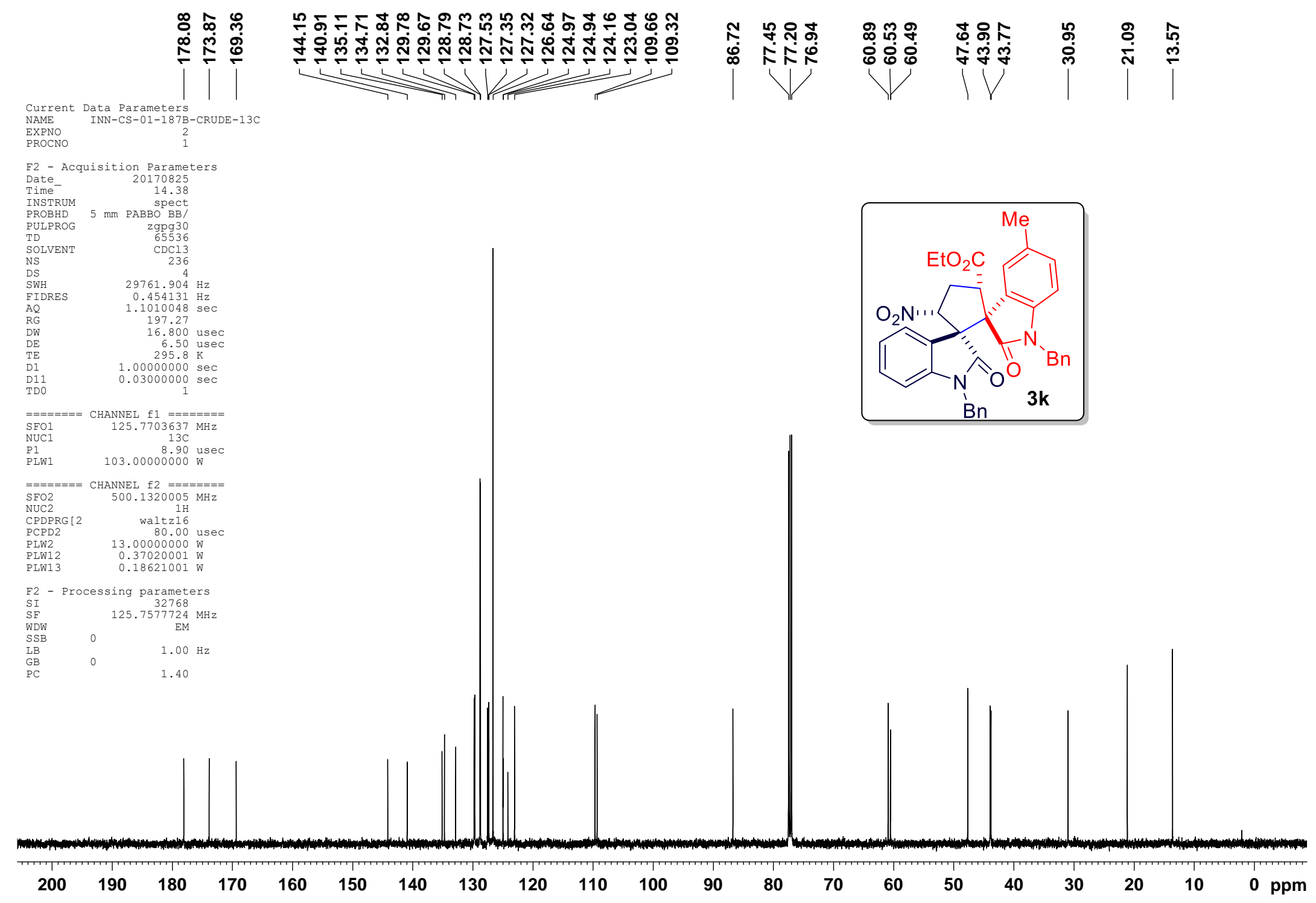

Figure S54. ${ }^{13} \mathrm{C}$ NMR $\left(125 \mathrm{MHz}, \mathrm{CDCl}_{3}\right)$ Spectrum of $\mathbf{3 k}$ 

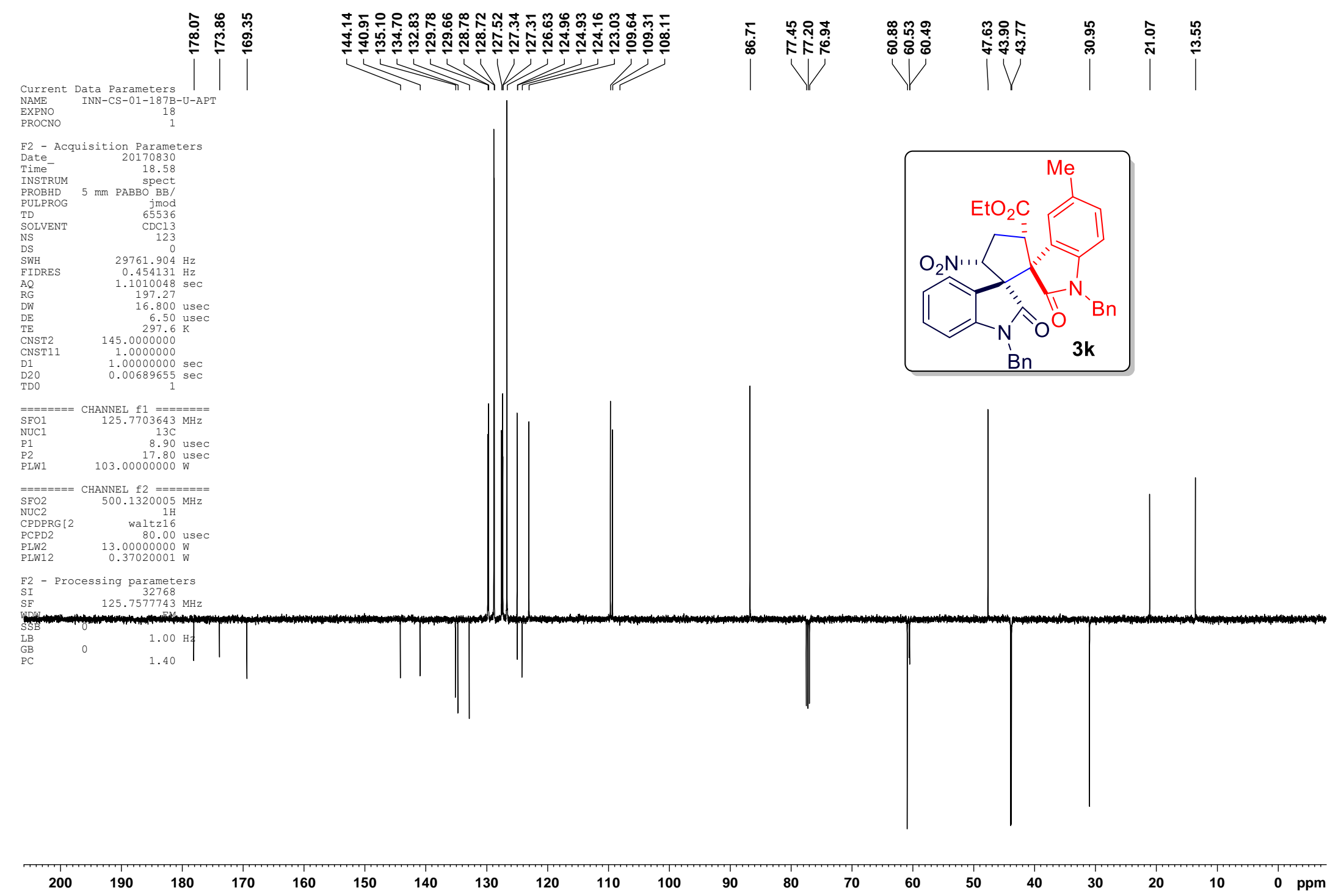

Figure S55. ${ }^{13} \mathrm{C}-\mathrm{APT}$ NMR $\left(125 \mathrm{MHz}, \mathrm{CDCl}_{3}\right)$ Spectrum of $\mathbf{3 k}$ 

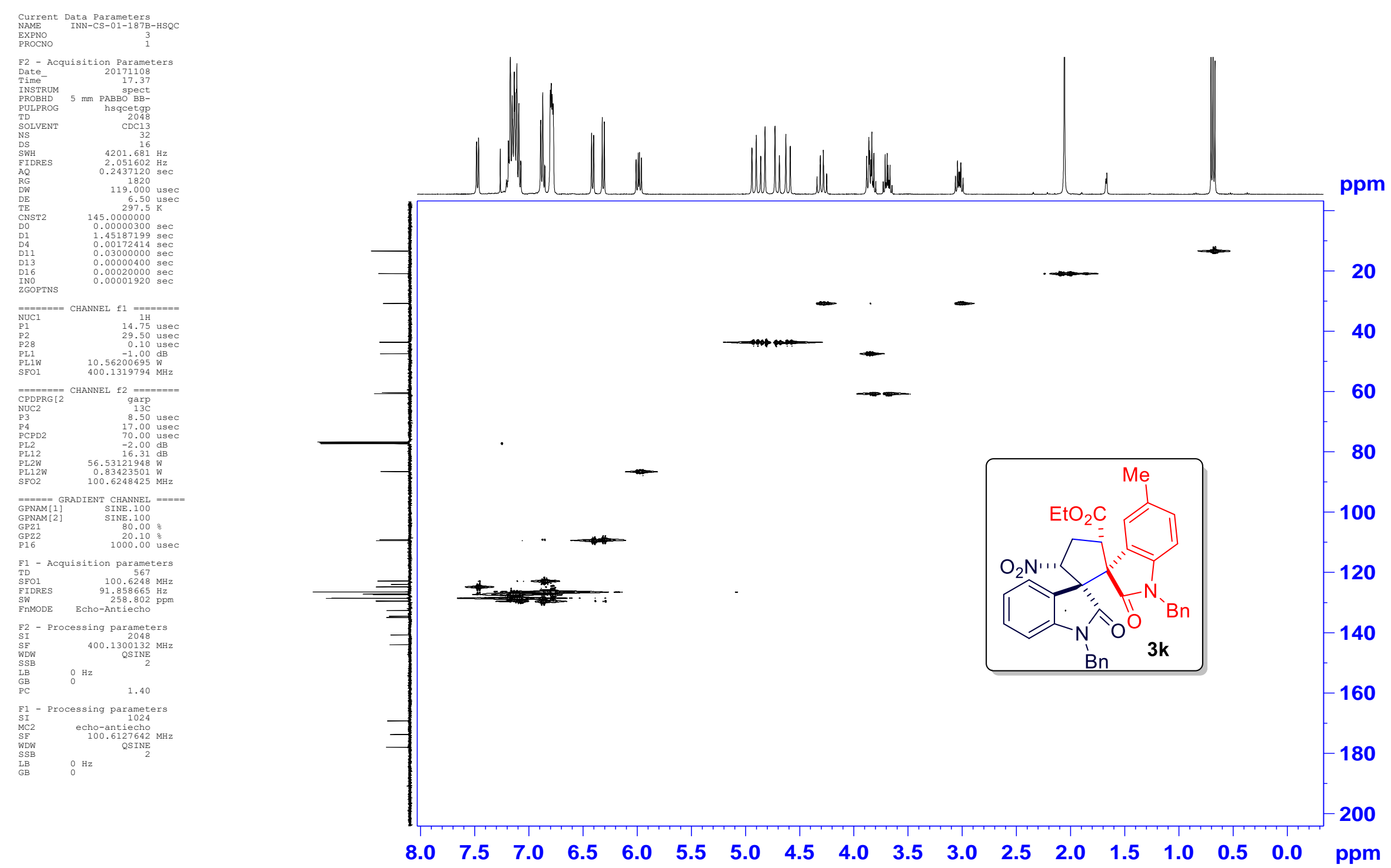

Figure S56. ${ }^{1} \mathrm{H}-{ }^{13} \mathrm{C}$ HSQC NMR $\left(100 \mathrm{MHz}, \mathrm{CDCl}_{3}\right)$ Spectrum of $\mathbf{3 k}$ 

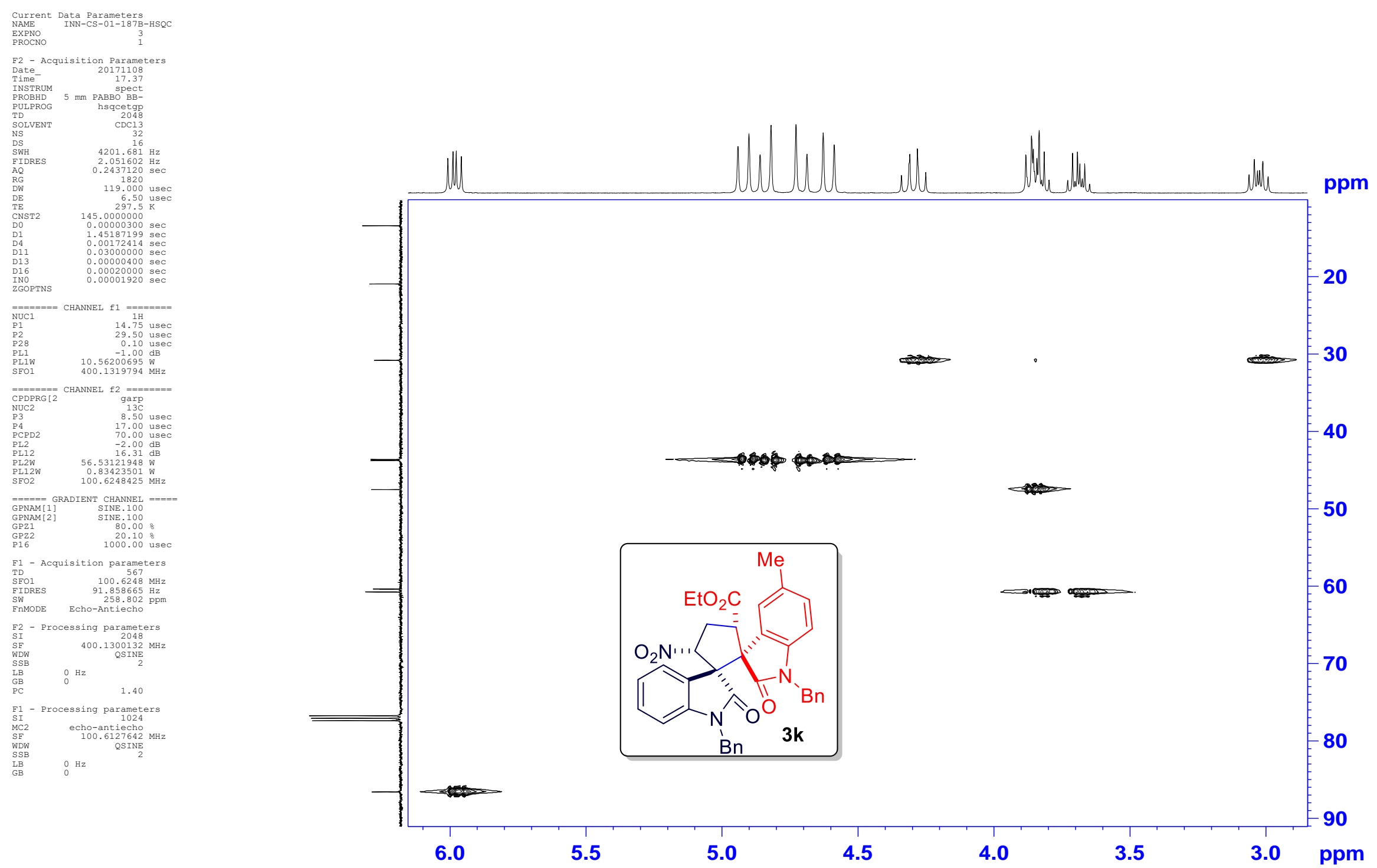

Figure S57. $1 \mathrm{H}-{ }^{13} \mathrm{C}$ HSQC NMR (100 MHz, $\left.\mathrm{CDCl}_{3}\right)$ Spectrum (Expansion) of $\mathbf{3 k}$ 


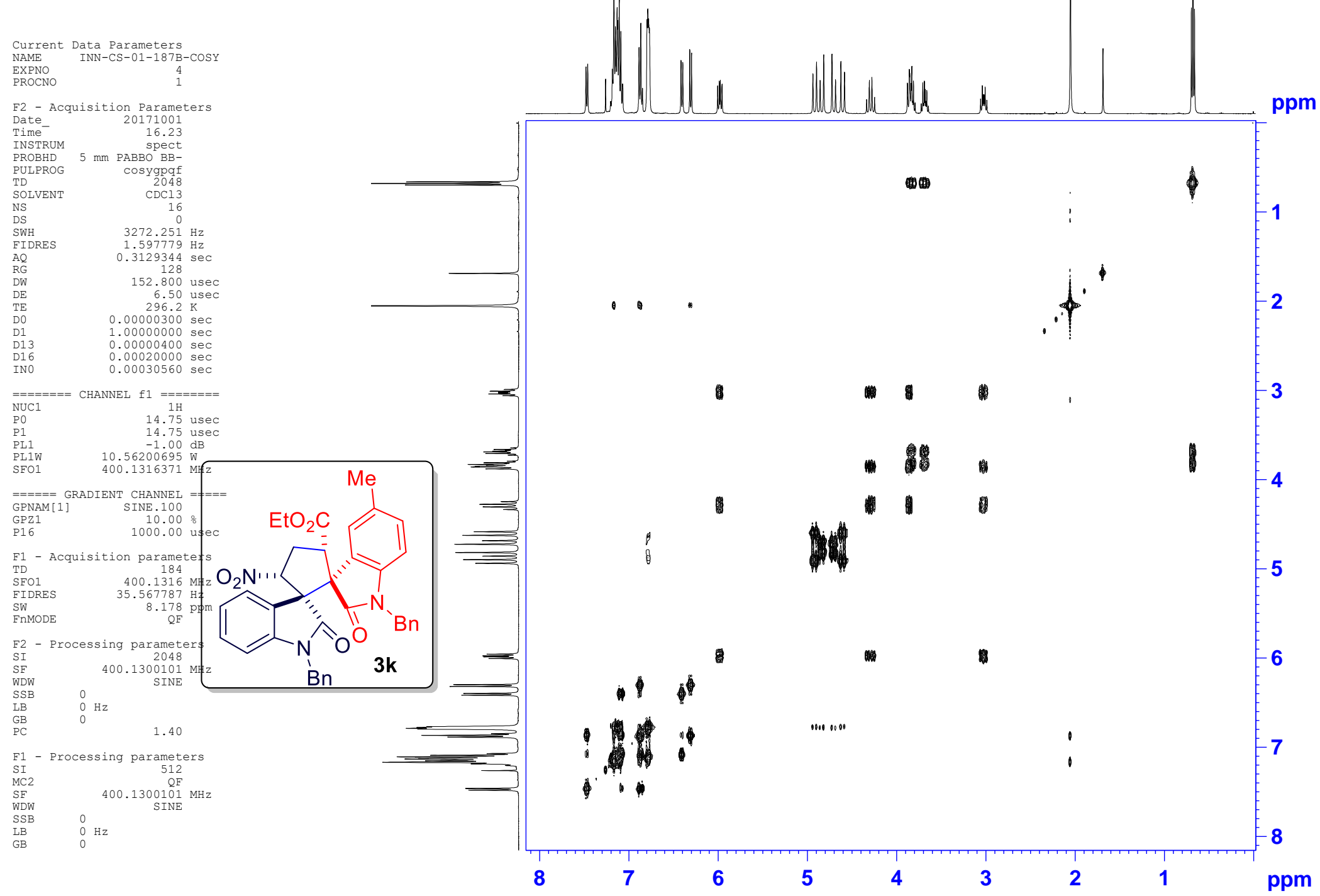

Figure S58. ${ }^{1} \mathrm{H}-{ }^{1} \mathrm{H}$ COSY NMR (400 MHz, $\left.\mathrm{CDCl}_{3}\right)$ Spectrum of $\mathbf{3 k}$ 


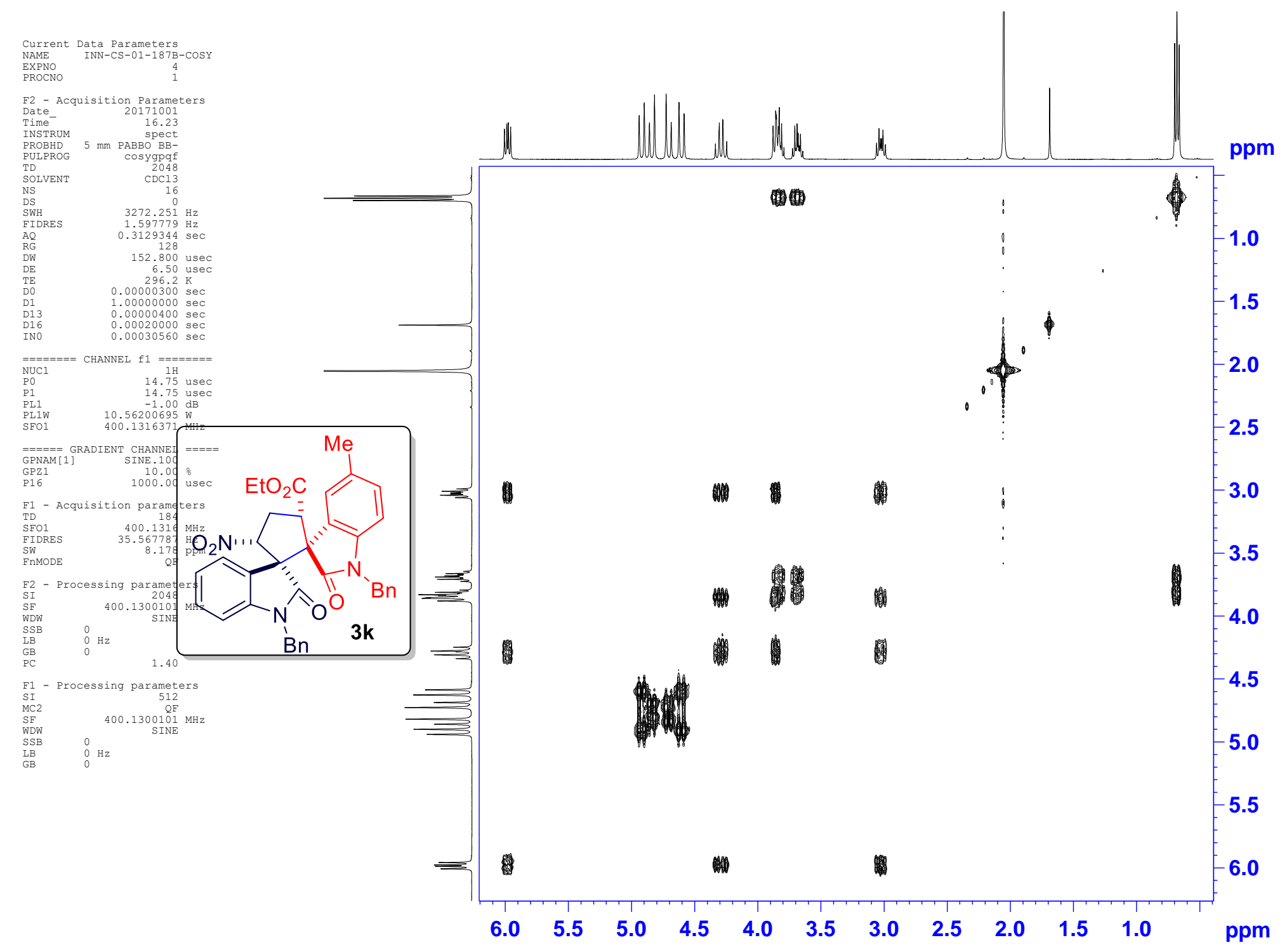

Figure S59. ${ }^{1} \mathrm{H}-{ }^{1} \mathrm{H}$ COSY NMR (400 MHz, $\mathrm{CDCl}_{3}$ ) Spectrum (Expansion) of $\mathbf{3 k}$ 

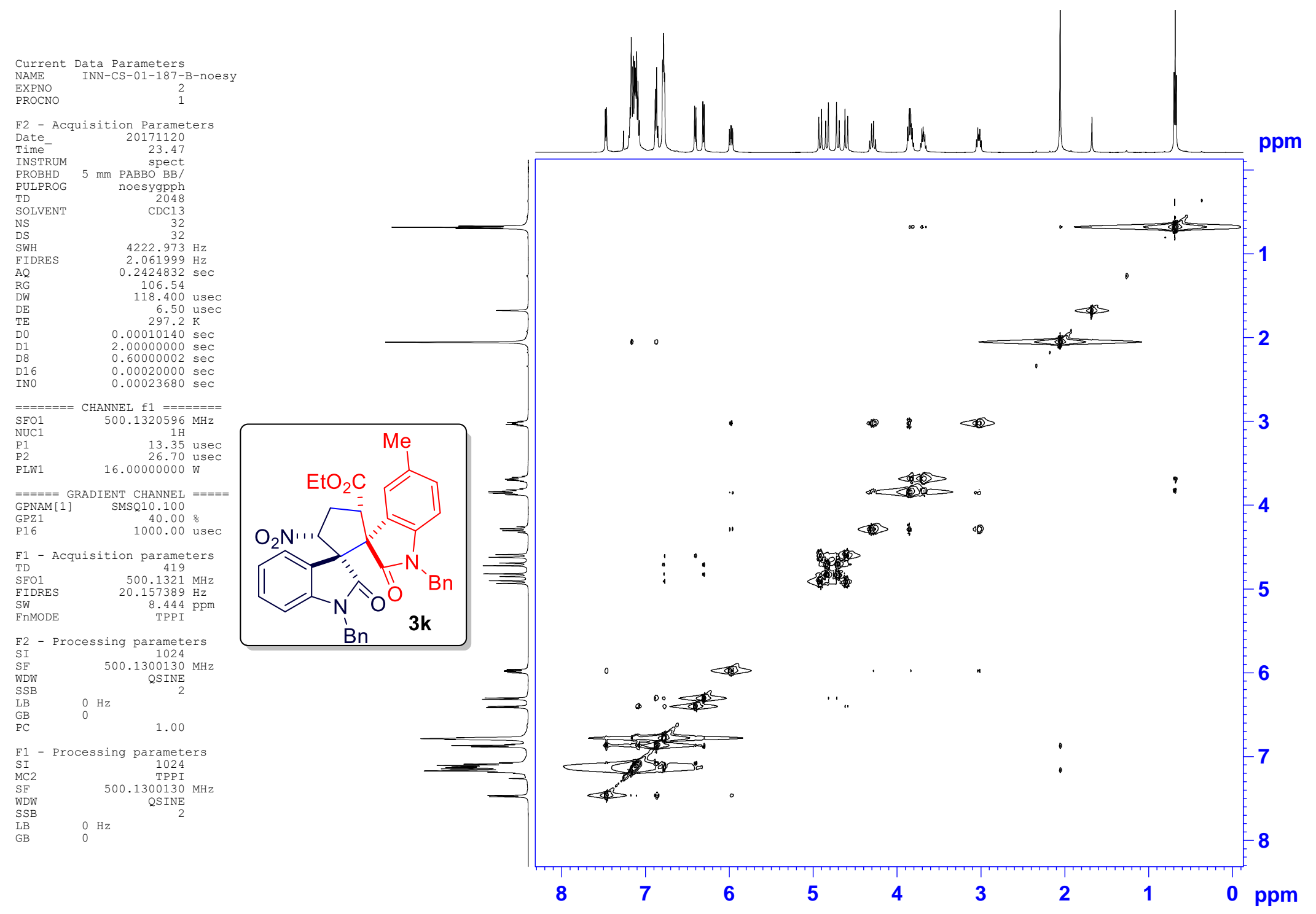

Figure S60. ${ }^{1} \mathrm{H}-{ }^{1} \mathrm{H}$ NOESY NMR $\left(500 \mathrm{MHz}, \mathrm{CDCl}_{3}\right)$ Spectrum of $\mathbf{3 k}$ 


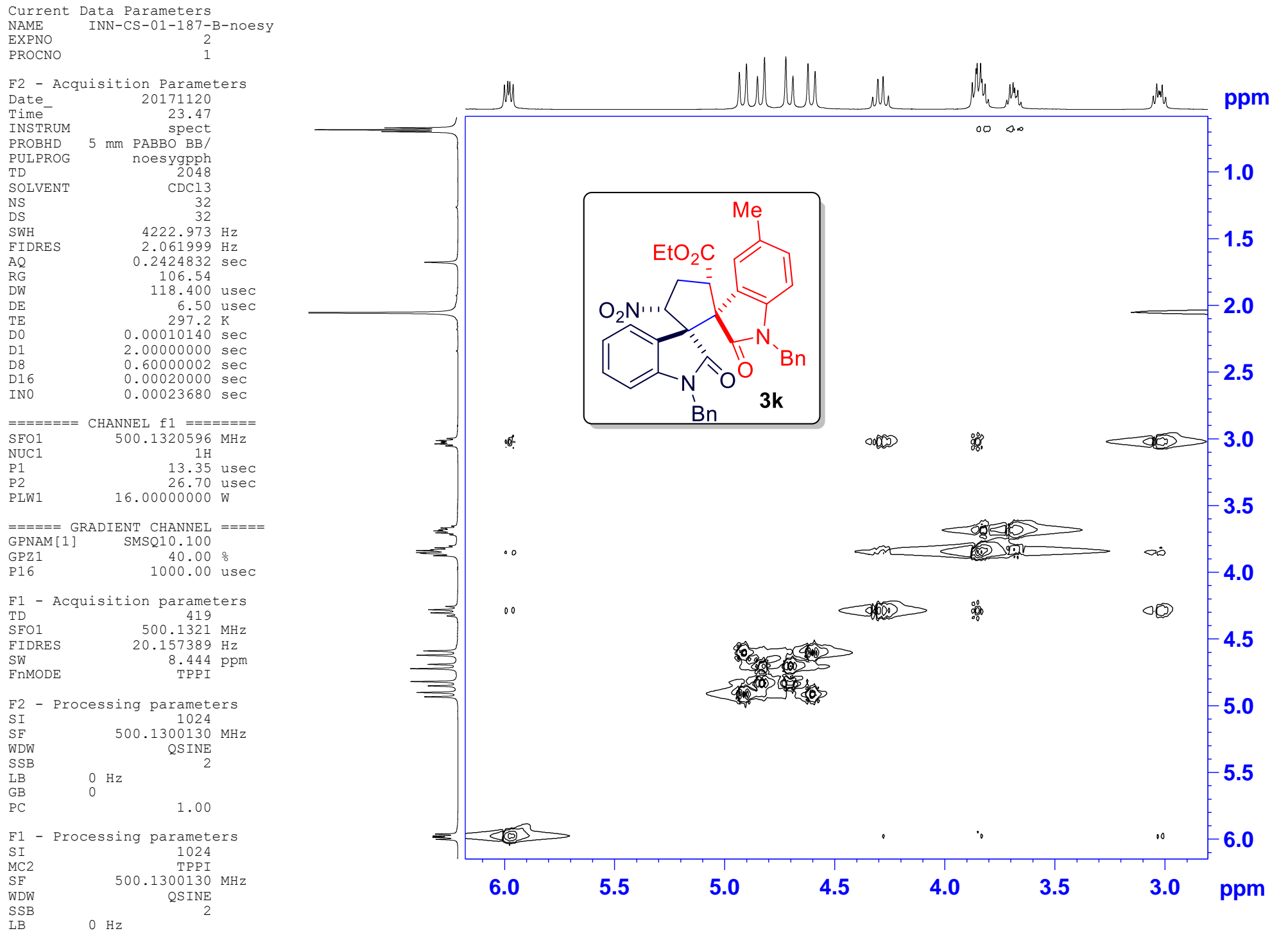

Figure S61. ${ }^{1} \mathrm{H}-{ }^{1} \mathrm{H}$ NOESY NMR (500 MHz, $\left.\mathrm{CDCl}_{3}\right)$ Spectrum (Expansion) of 3k 


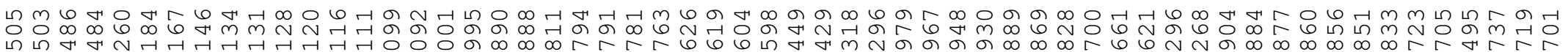
.

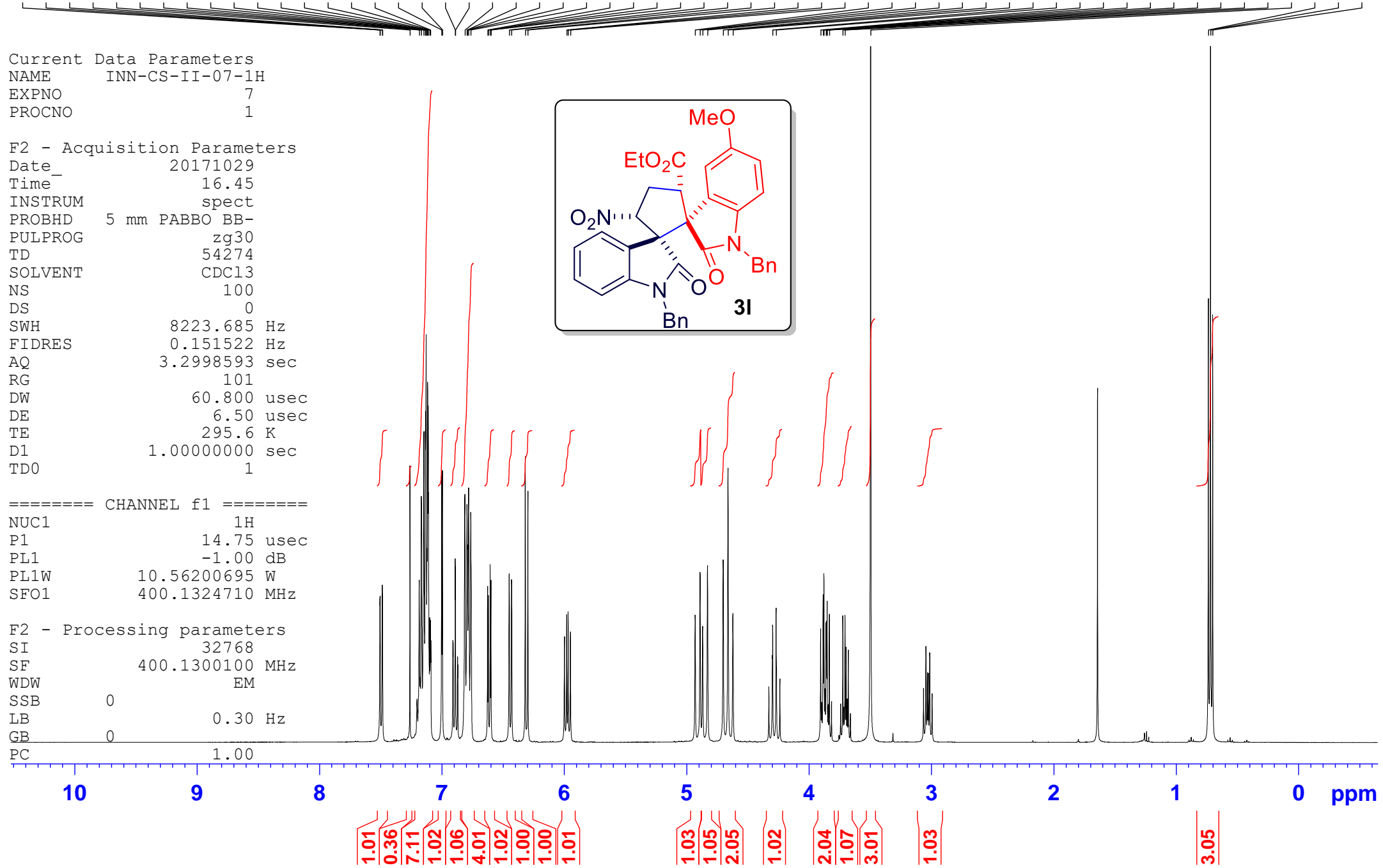

Figure S62. ${ }^{1} \mathrm{H}$ NMR $\left(400 \mathrm{MHz}, \mathrm{CDCl}_{3}\right)$ Spectrum of $\mathbf{3 l}$ 

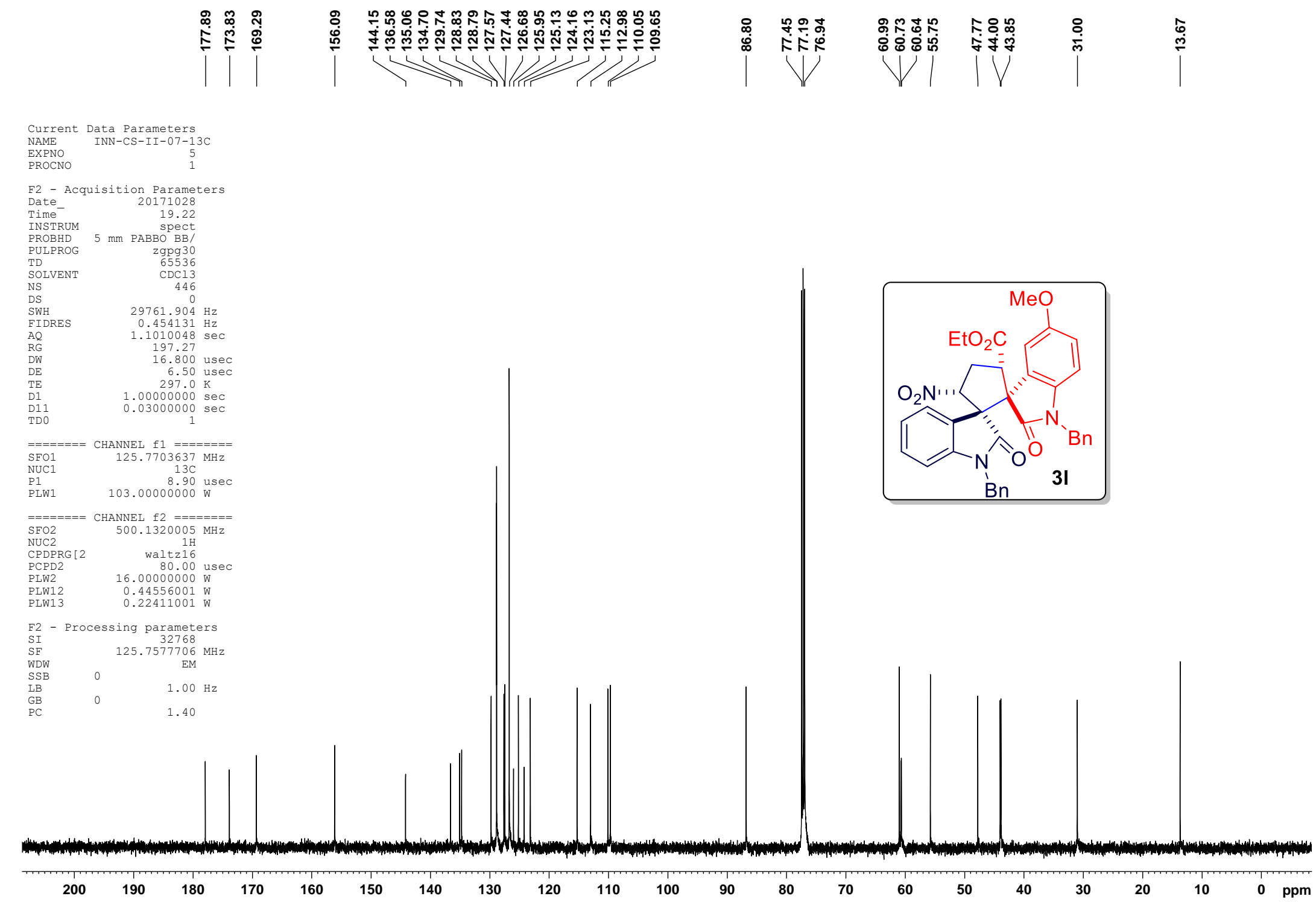

Figure $\mathbf{S 6 3} .{ }^{13} \mathrm{C} \mathrm{NMR}\left(125 \mathrm{MHz}, \mathrm{CDCl}_{3}\right)$ Spectrum of $\mathbf{3 l}$ 

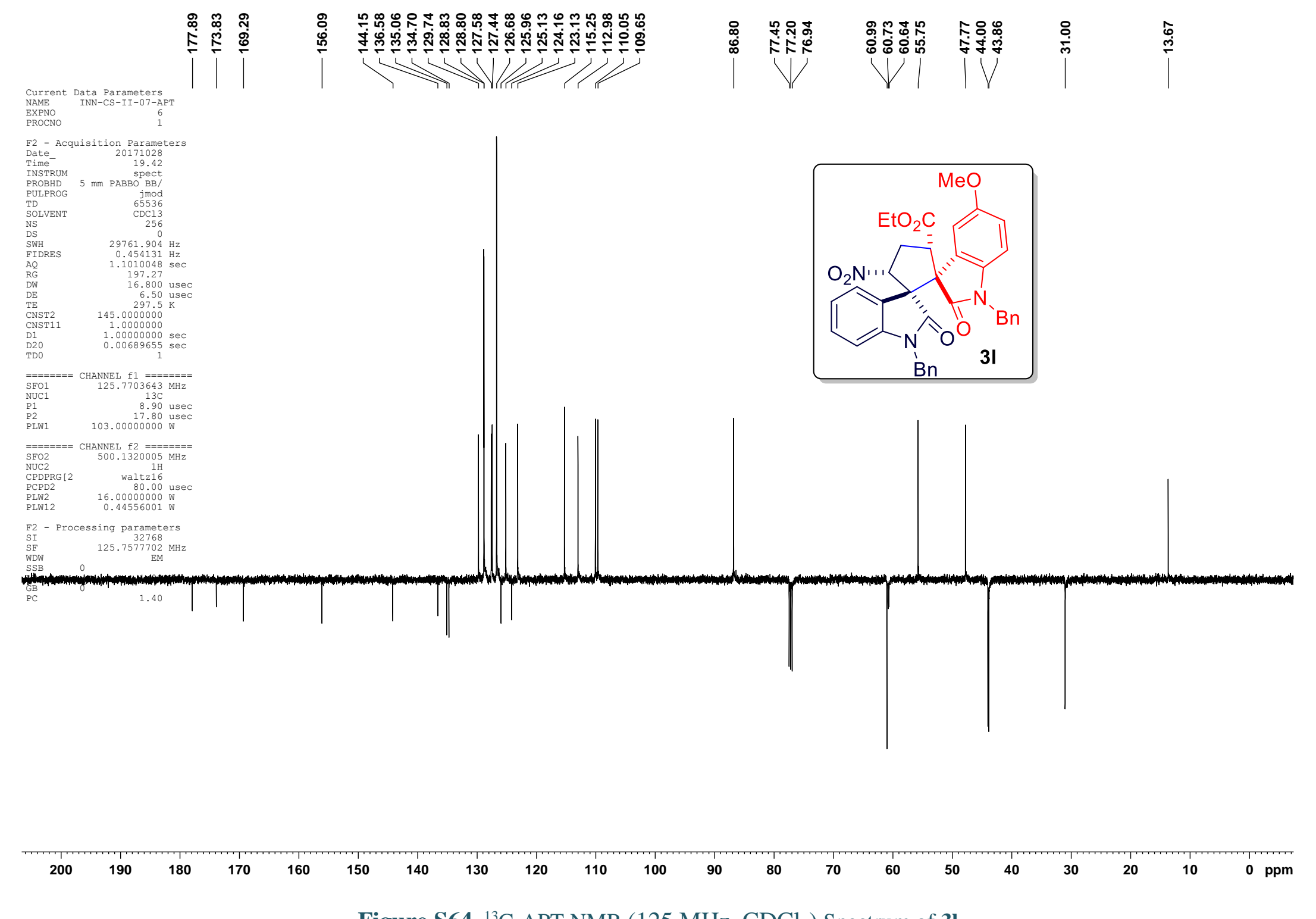

Figure S64. ${ }^{13} \mathrm{C}$-APT NMR (125 MHz, $\left.\mathrm{CDCl}_{3}\right)$ Spectrum of $\mathbf{3 l}$ 


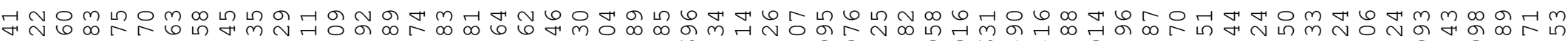
罂 $\therefore \dot{r}-\dot{r} \therefore \dot{r}$

Current Data Parameters

NAME INN-CS-01-188A-1H

EXPNO

1
1

F2 - Acquisition Parameters

Date_r 20171007

$\begin{array}{lr}\text { Time } & - \\ \text { INSTRUM } & 11.48\end{array}$

spect
INSTRUM
PROBHD $5 \mathrm{~mm}$ PABBO BB-

PULPROG
zq30

TD 54274

SOLVENT

NS

SWH

FIDRES

$\mathrm{AQ}$

$\begin{array}{lr}\text { RG } & 64 \\ \text { DW } & 60.800 \text { usec }\end{array}$

DE $\quad 6.50$ usec

$296.2 \mathrm{~K}$

TDO 1.0000000

$=======$ CHANNEL $\mathrm{f} 1 \mathrm{l}========$

NUC

P1

PL1

PL1W
SFO1

14.75 usec

$10.56200695 \mathrm{~d}$

F2 - Processing parameters

SI 32768

$\begin{array}{lr}\text { SI } & 32768 \\ \text { SF } & 400.1300101 \\ \text { MHz }\end{array}$

$\begin{array}{lr}\text { SE } & 400.1300101 \\ \text { WDW } & \text { EM }\end{array}$

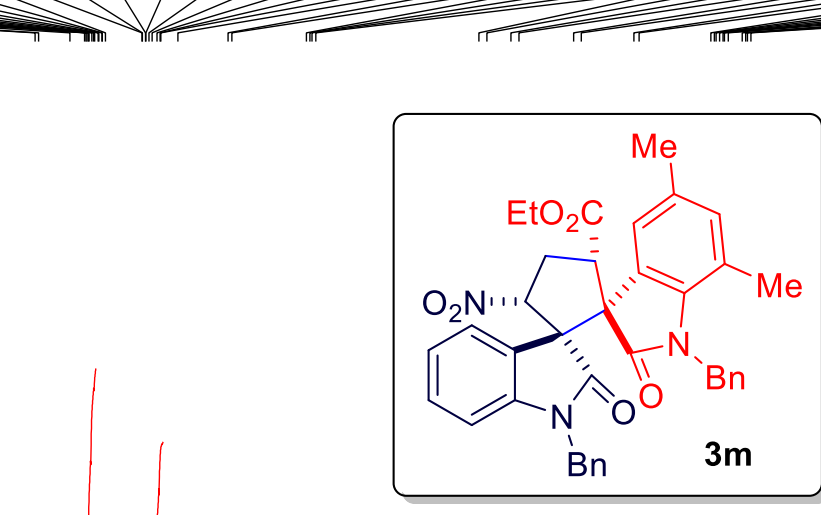

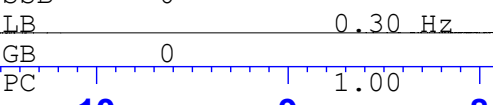

10

9

8

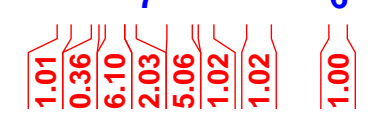

5

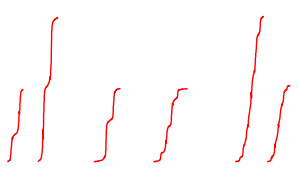

Figure S65. ${ }^{1} \mathrm{H}$ NMR (400 $\mathbf{M H z}, \mathrm{CDCl}_{3}$ ) Spectrum of $\mathbf{3 m}$ 


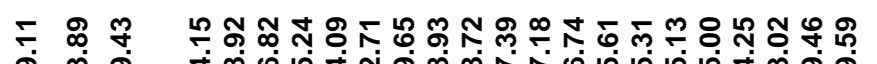

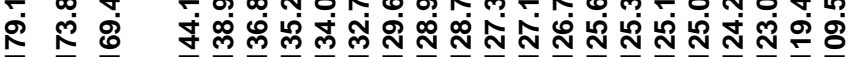

NAME
EXPNO
PROCNO

$\mid$

Current Data Parameters
NAME
EXPN
INN-CS- $01-188 \mathrm{~A}-13 \mathrm{C}$
XXNO
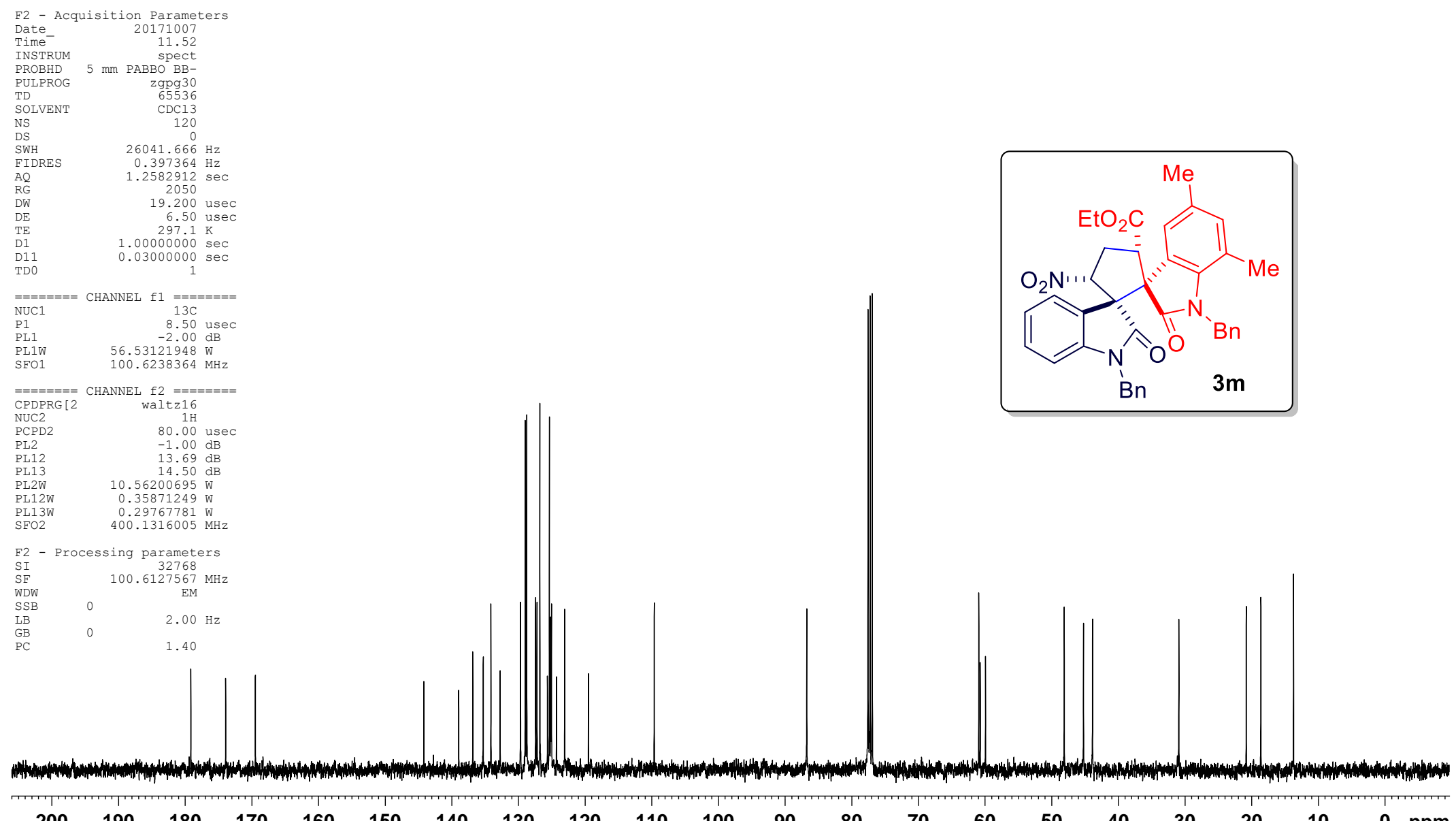

Figure S66. ${ }^{13} \mathrm{C}$ NMR $\left(100 \mathrm{MHz}, \mathrm{CDCl}_{3}\right)$ Spectrum of $\mathbf{3 m}$

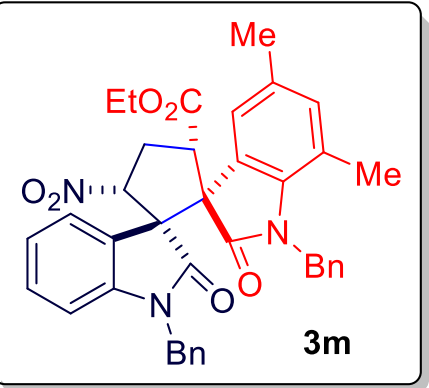




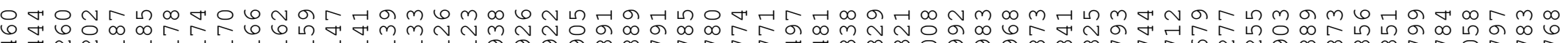
H rarrarrarrarra

Current Data Parameters

NAME INN-CS-II-11-A-1H

EXPNO

F2 - Acquisition Parameters

Date_ 20171124

spect
INSTRUM

$\begin{array}{lr}\text { PULPROG } & \text { Z930 } \\ \text { TD } & 65536\end{array}$

SOLVENT

NS

DWH

$\begin{array}{ll}0 & 0000.000 \mathrm{~Hz}\end{array}$

$0.152588 \mathrm{~Hz}$
3.2767999

RG

$\begin{array}{lr}\text { DW } & 50.000 \text { usec } \\ \text { DE } & 6.50 \text { usec }\end{array}$

$\begin{array}{lr}\text { DE } & 6.50 \text { usec } \\ \text { TE } & 297.0 \mathrm{~K}\end{array}$

D1 $\quad 1.00000000 \mathrm{sec}$

$=======$ CHANNEL $\mathrm{f1}========$

SFO1 $500.1330885 \mathrm{MHz}$

NUC1 1 1H

P1
PLW1 $\quad 16.00000000 \mathrm{~W}$

F2 - Processing parameters

SI $\quad 65536$

WDW

$\begin{array}{lll}\text { WDW } & \text { EM } \\ \text { SSB } & 0 & 0.30 \mathrm{~Hz}\end{array}$

$\begin{array}{lll}\text { LB } & 0 & \\ \text { GB } & 0 & 1.00\end{array}$

(1.00

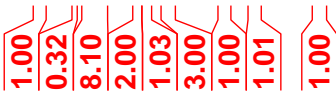

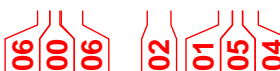

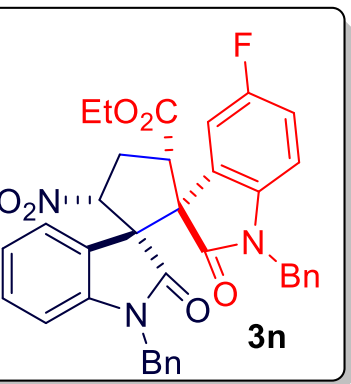

$\pi$

Figure S67. ${ }^{1} \mathrm{H}$ NMR (500 MHz, $\left.\mathrm{CDCl}_{3}\right)$ Spectrum of $\mathbf{3 n}$ 

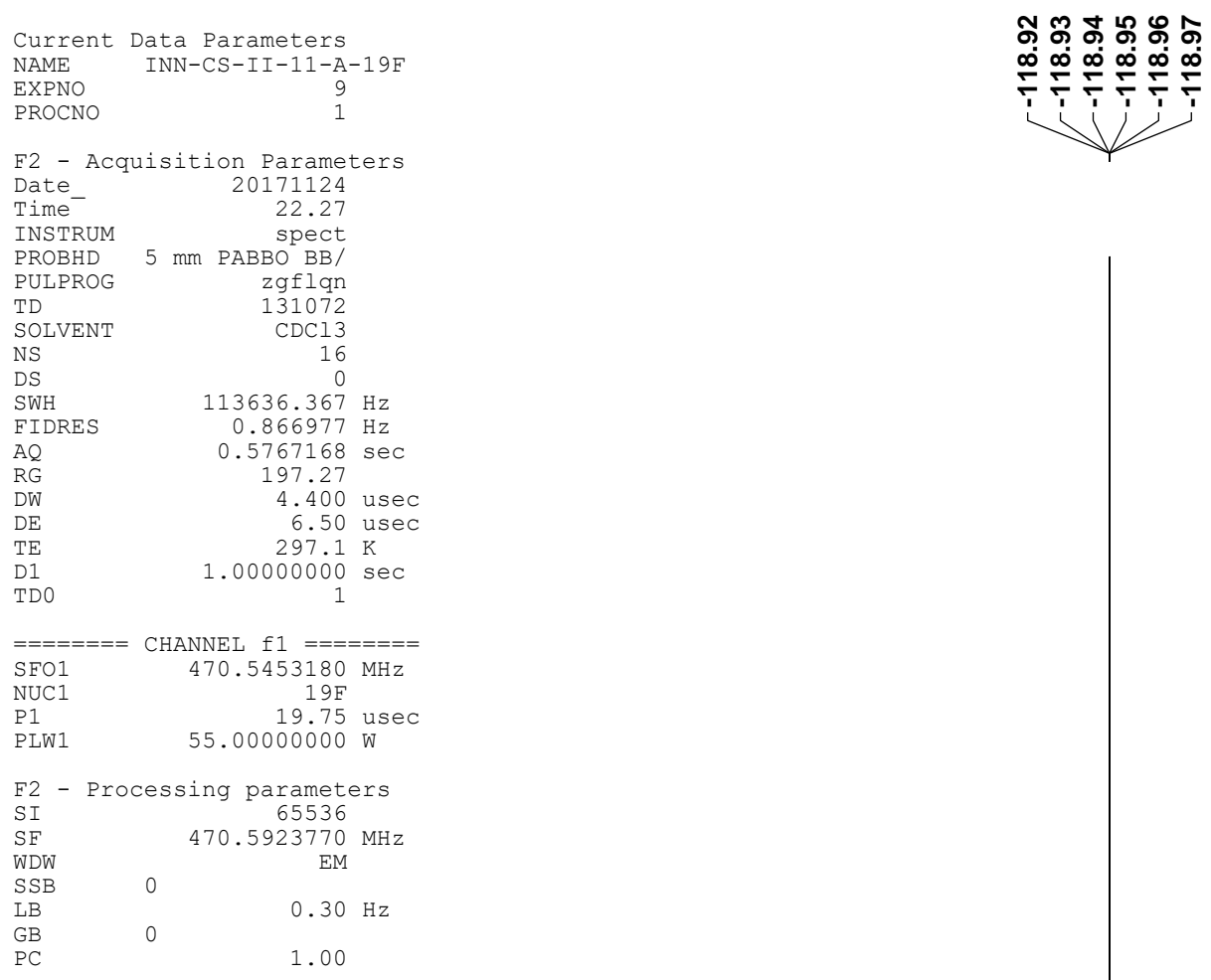

F2 - Acquisition Parameters

$\begin{array}{lr}\text { Date } & 20171124 \\ \text { Time } & 22.27\end{array}$

spect
NNSTRUM $5 \mathrm{~mm}$ PABBO BB

$13636.367 \mathrm{~Hz}$ $.5767168 \mathrm{~Hz}$

4.400 usec

6.50 usec

$1.00000000 \mathrm{Ksec}$

$=======$ CHANNEL $\mathrm{fl}=======$
SFO1

NUC1 $\quad 19 \mathrm{~F}$

$55.00000000 \mathrm{~W}$

F2 - Processing parameters

SF $\quad 470.5923770 \mathrm{MHz}$

SSB 0

$\begin{array}{lll}\mathrm{GB} & 0 & 1.00\end{array}$
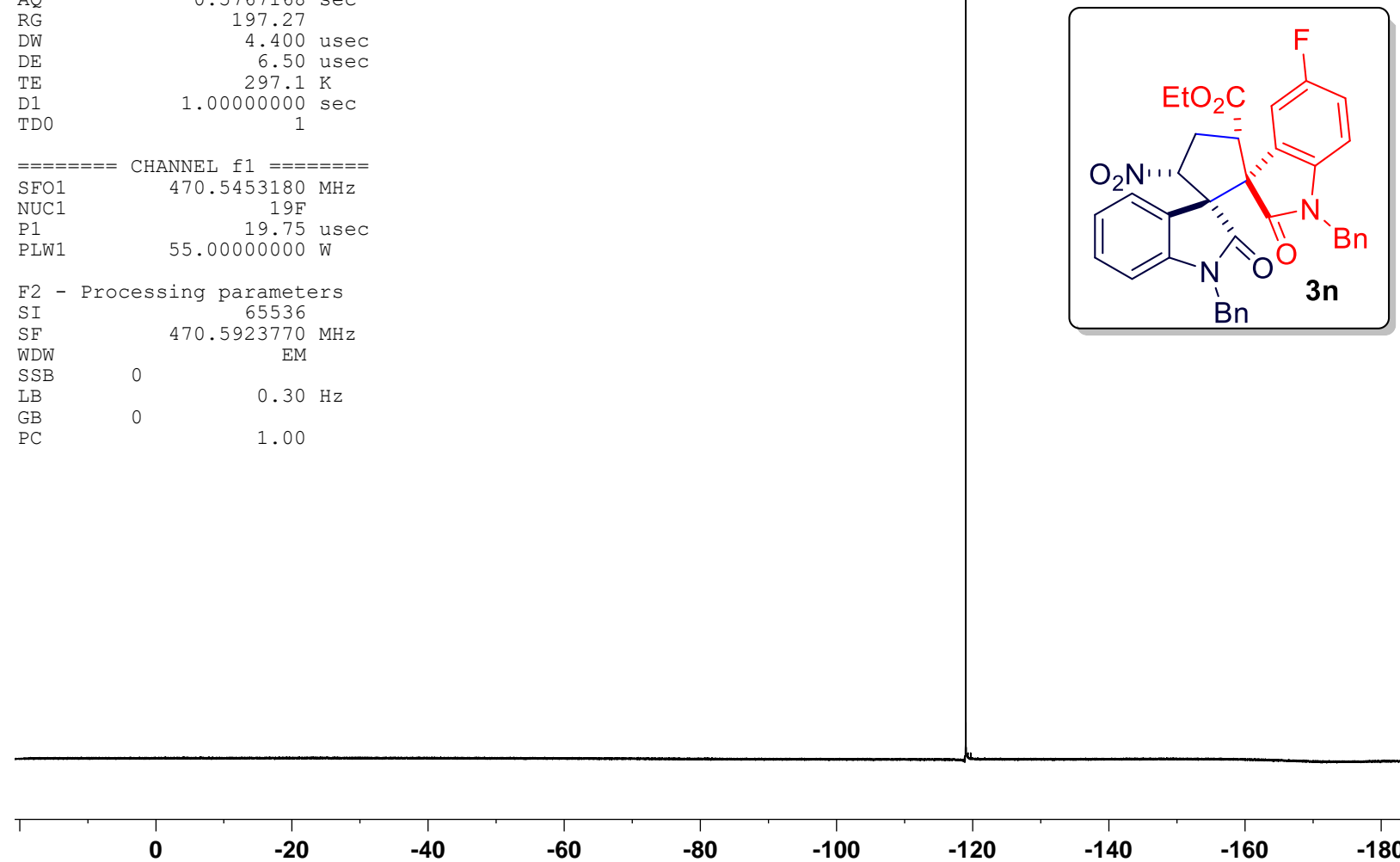

$-40$

$-60$

Figure S68. ${ }^{19} \mathrm{~F}$ NMR $\left(470 \mathrm{MHz}, \mathrm{CDCl}_{3}\right)$ Spectrum of $\mathbf{3 n}$ 


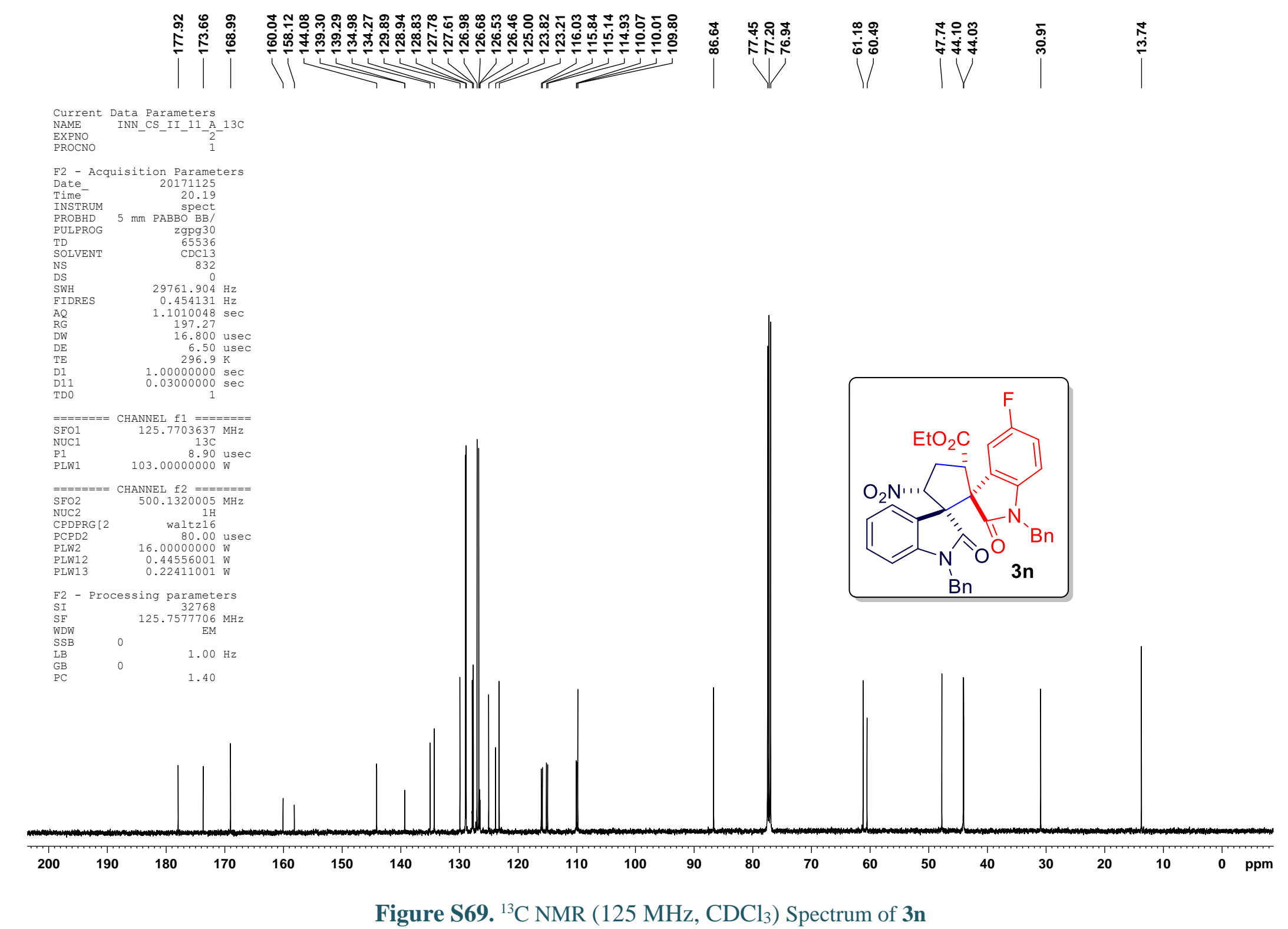




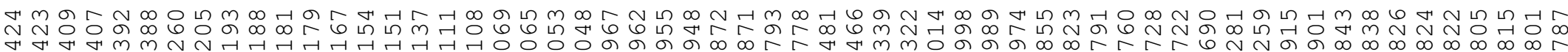

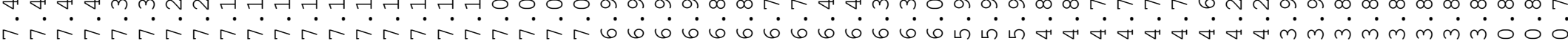

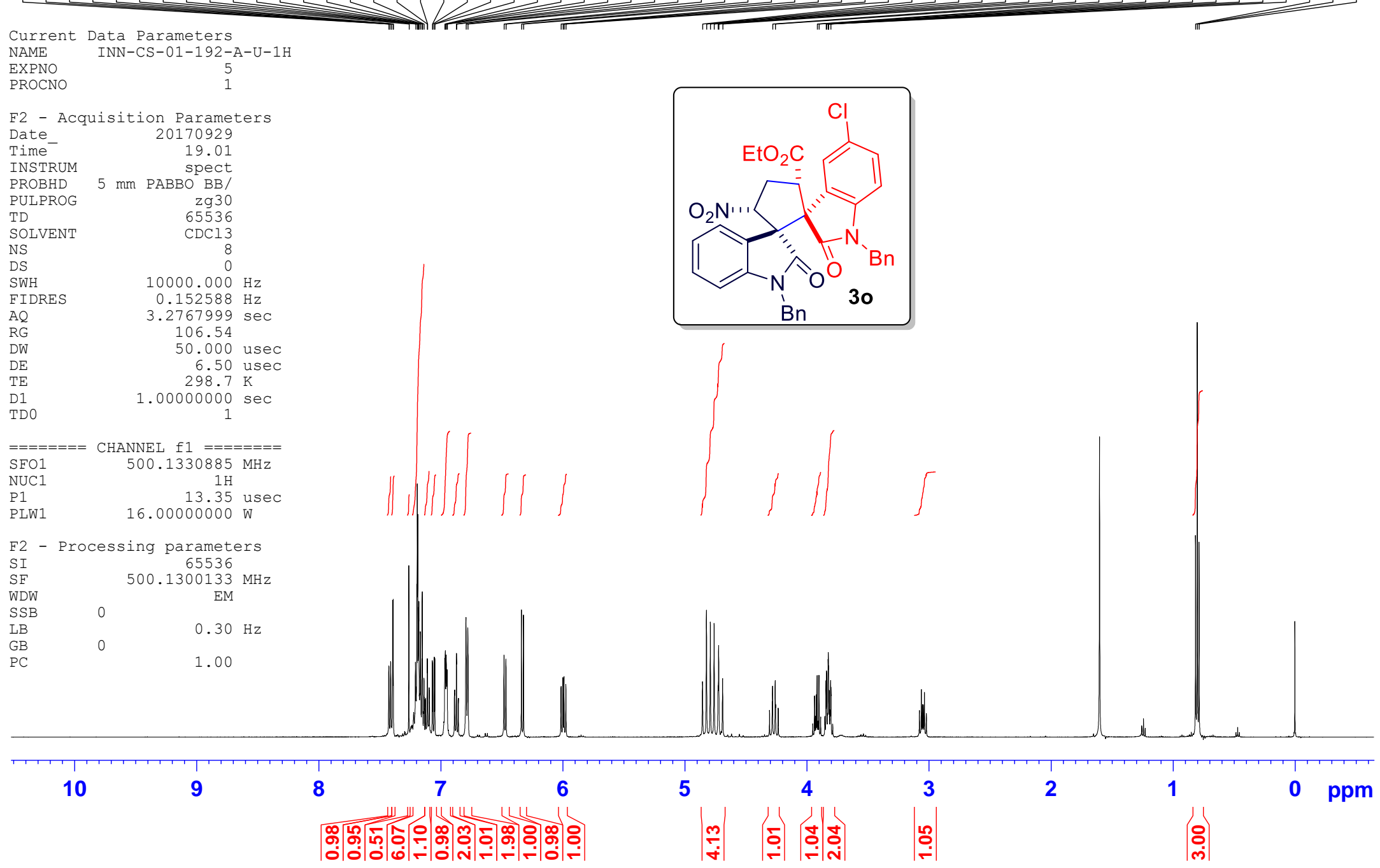

Figure S70. ${ }^{1} \mathrm{H}$ NMR $\left(500 \mathrm{MHz}, \mathrm{CDCl}_{3}\right)$ Spectrum of 30 


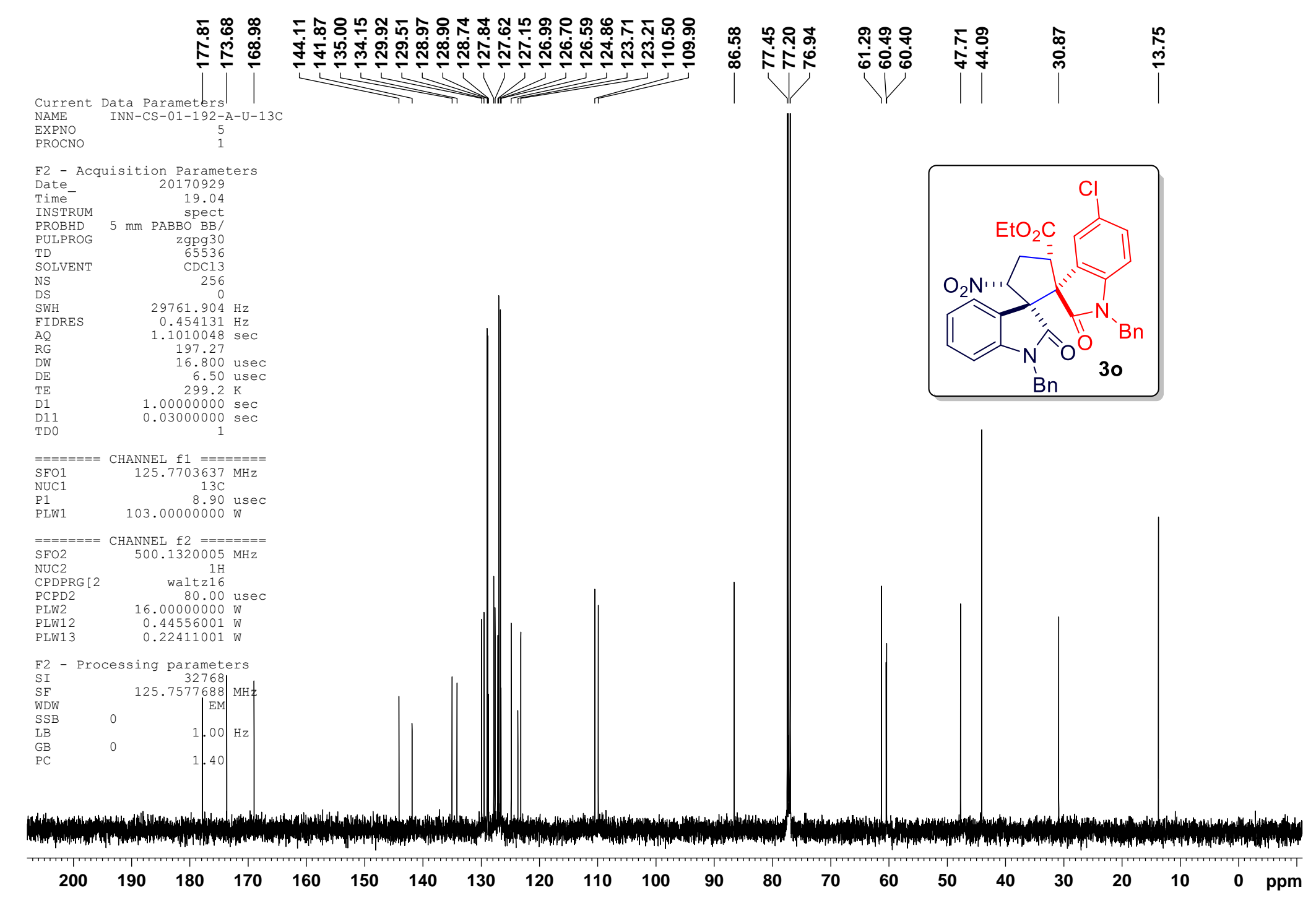

Figure S71. ${ }^{13} \mathrm{C}$ NMR $\left(125 \mathrm{MHz}, \mathrm{CDCl}_{3}\right)$ Spectrum of 30 

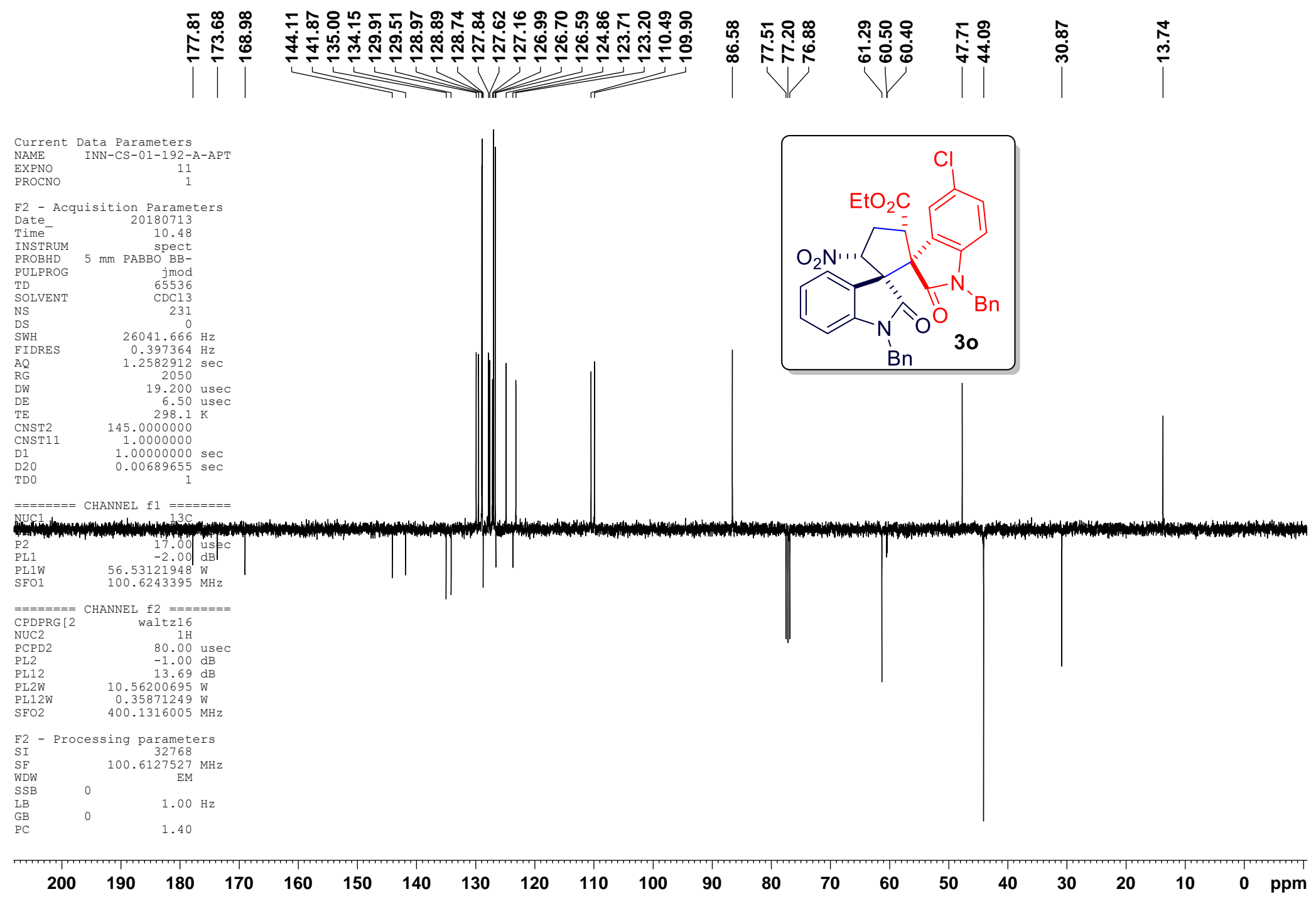

Figure S72. ${ }^{13} \mathrm{C}-\mathrm{APT}$ NMR (100 MHz, $\left.\mathrm{CDCl}_{3}\right)$ Spectrum of $\mathbf{3 o}$ 


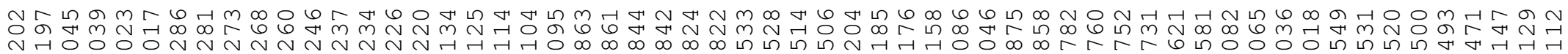

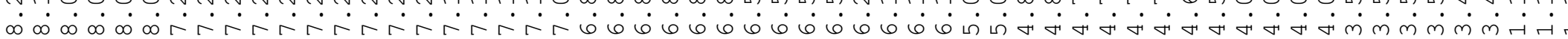
Parameters EXPNO

EROCNO

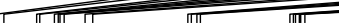

F2 - Acquisition Parameters

Date

Time $\quad 18.51$

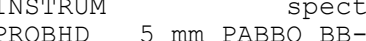

PROBHD

PULPROG

SOLVENT

NS

DS

SWH

AQ

RG

DE

TE

TDO

$=====$

zg 30
54274
$\mathrm{CDCl}$

16
0

$8223.685 \mathrm{~Hz}$ $3.2998593 \mathrm{sec}$

60.800 usec

HANNEL $\mathrm{f} 1===$

PL1

PL1W
SFO1

$400.1324710 \mathrm{MHz}$

F2 - Processing parameters

$\mathrm{SF}$

WDW

LB

32768
$400.1300100 \mathrm{MHz}$

EM MHz
.

$0.30 \mathrm{~Hz}$

1.00

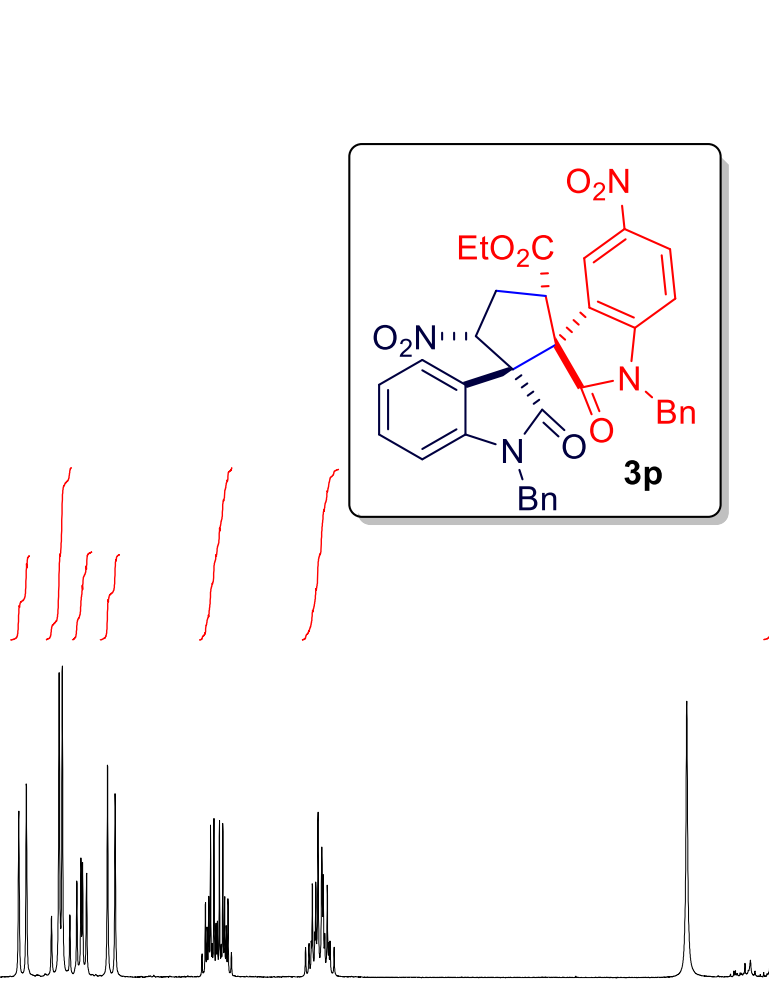

10

7

6

5

4

3

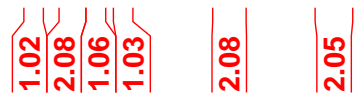

2

1

0 ppm

|

Figure S73. ${ }^{1} \mathrm{H} \mathrm{NMR}\left(400 \mathrm{MHz}, \mathrm{CDCl}_{3}\right)$ Spectrum of $\mathbf{3 p}$ 


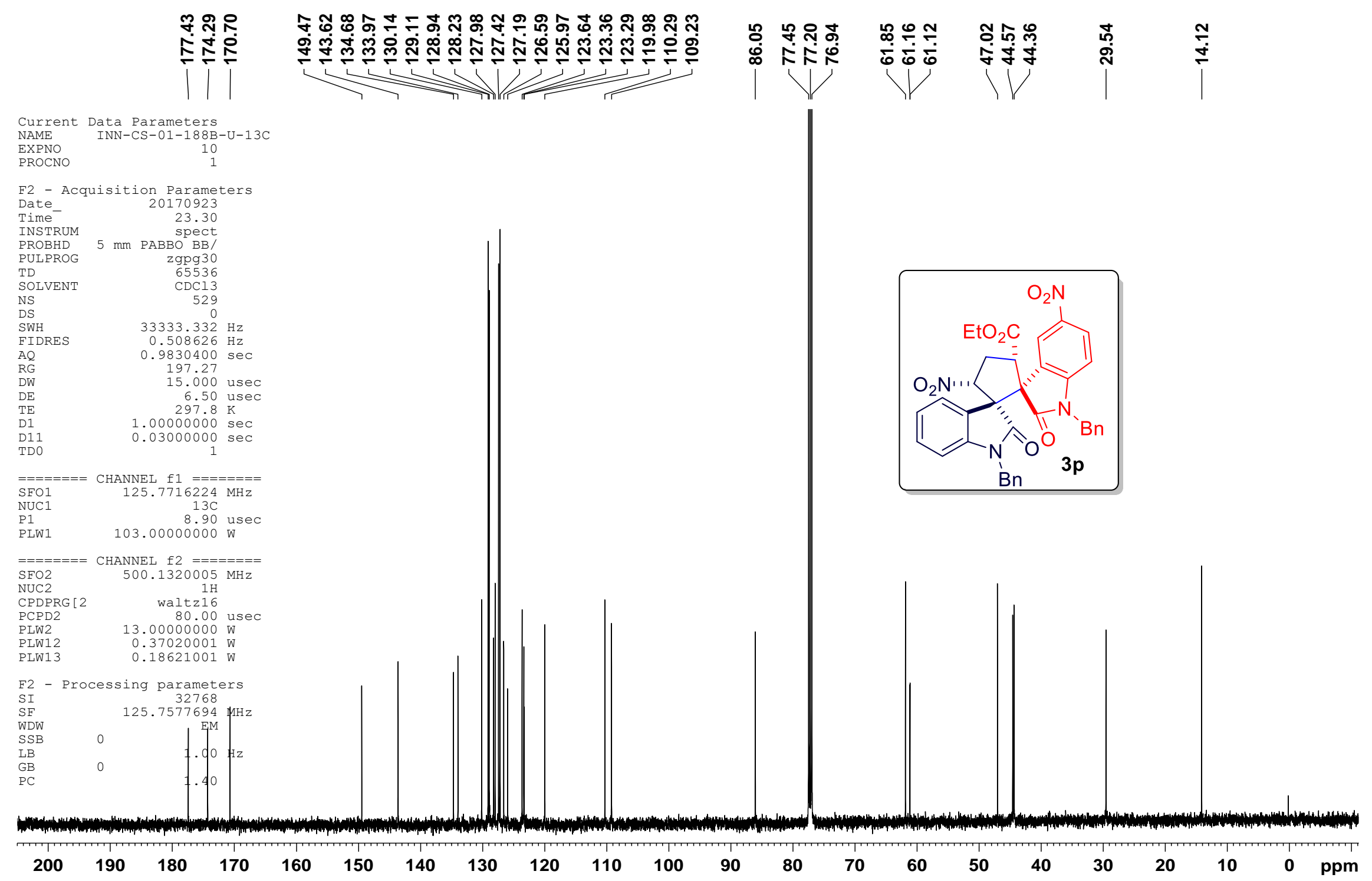

Figure S74. ${ }^{13} \mathrm{C}$ NMR (125 MHz, $\left.\mathrm{CDCl}_{3}\right)$ Spectrum of $\mathbf{3 p}$ 


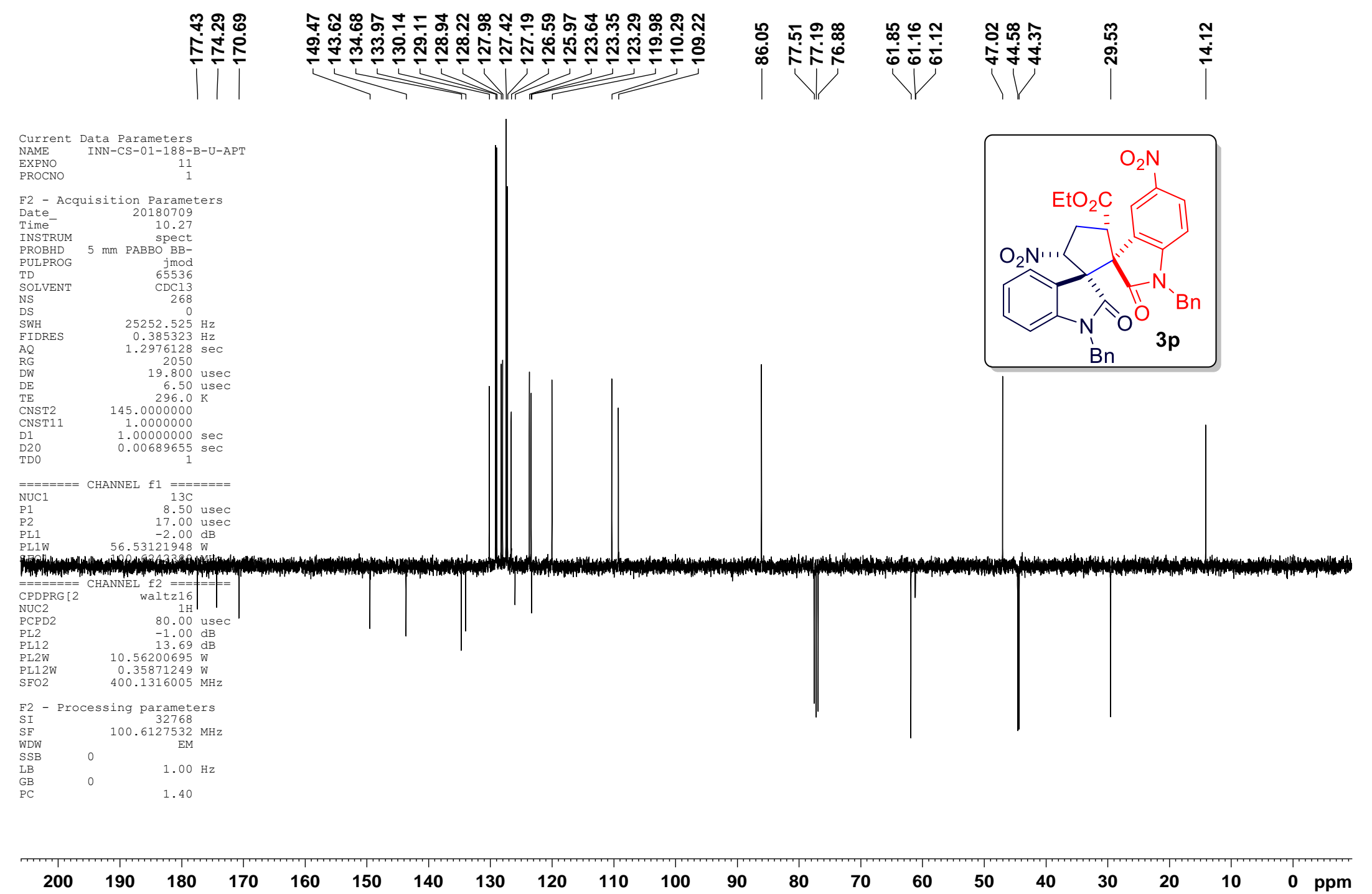

Figure S75. ${ }^{13} \mathrm{C}$-APT NMR (100 MHz, $\left.\mathrm{CDCl}_{3}\right)$ Spectrum of $\mathbf{3 p}$ 


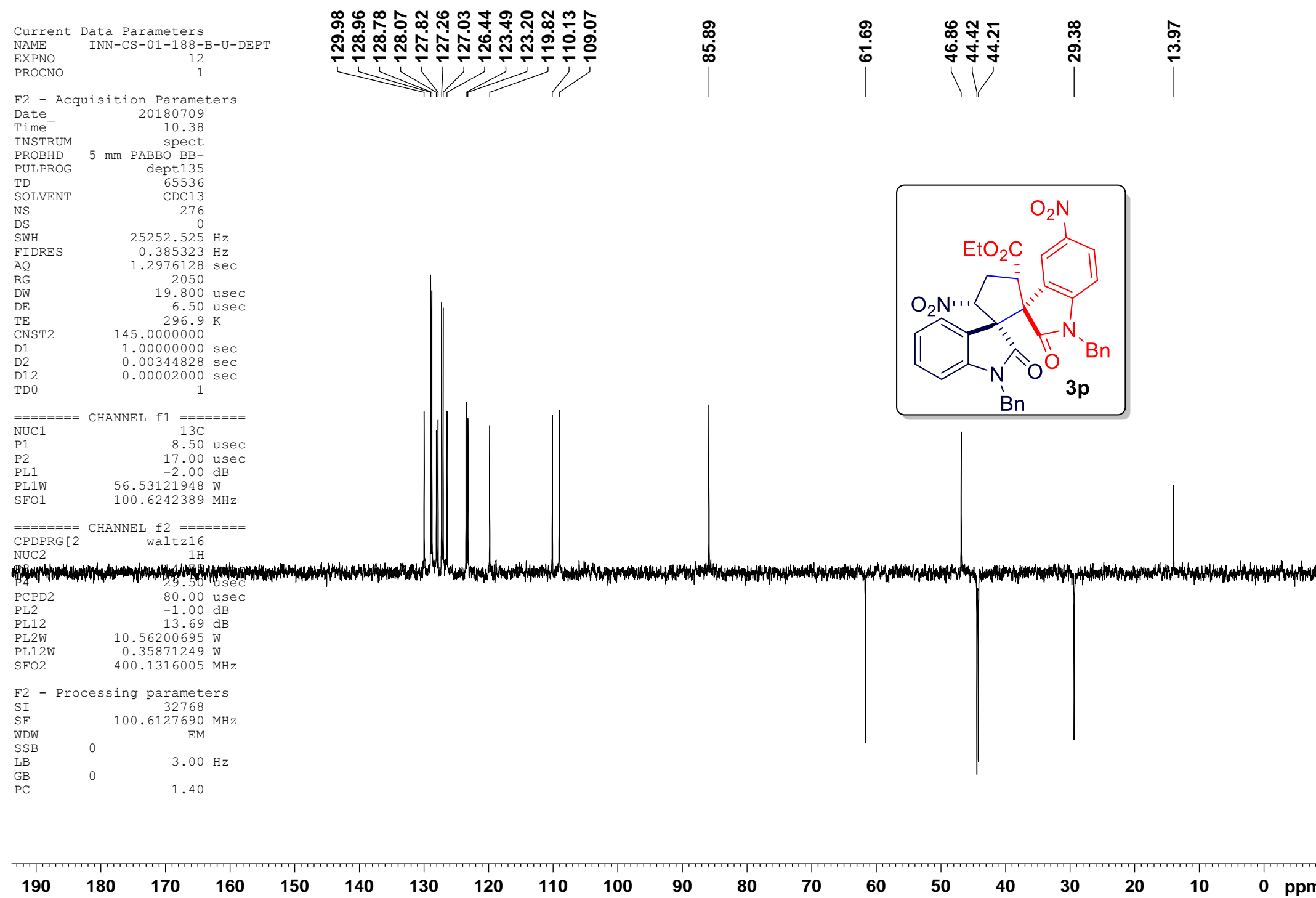

Figure S76. ${ }^{13} \mathrm{C}$-DEPT NMR (100 MHz, $\left.\mathrm{CDCl}_{3}\right)$ Spectrum of 3p 


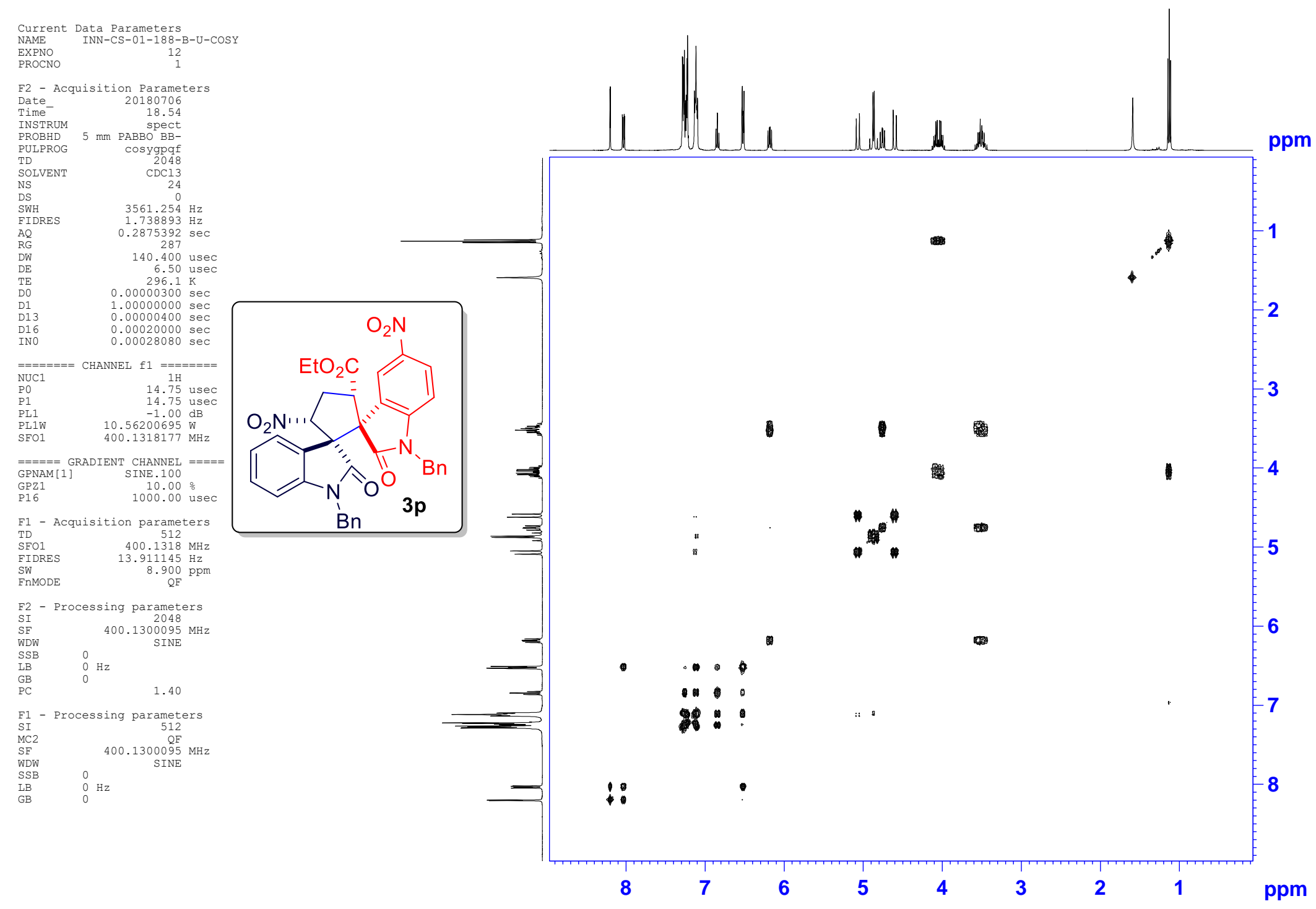

Figure S77. ${ }^{1} \mathrm{H}-{ }^{-1} \mathrm{H}$ COSY NMR (400 MHz, $\left.\mathrm{CDCl}_{3}\right)$ Spectrum of $\mathbf{3 p}$ 


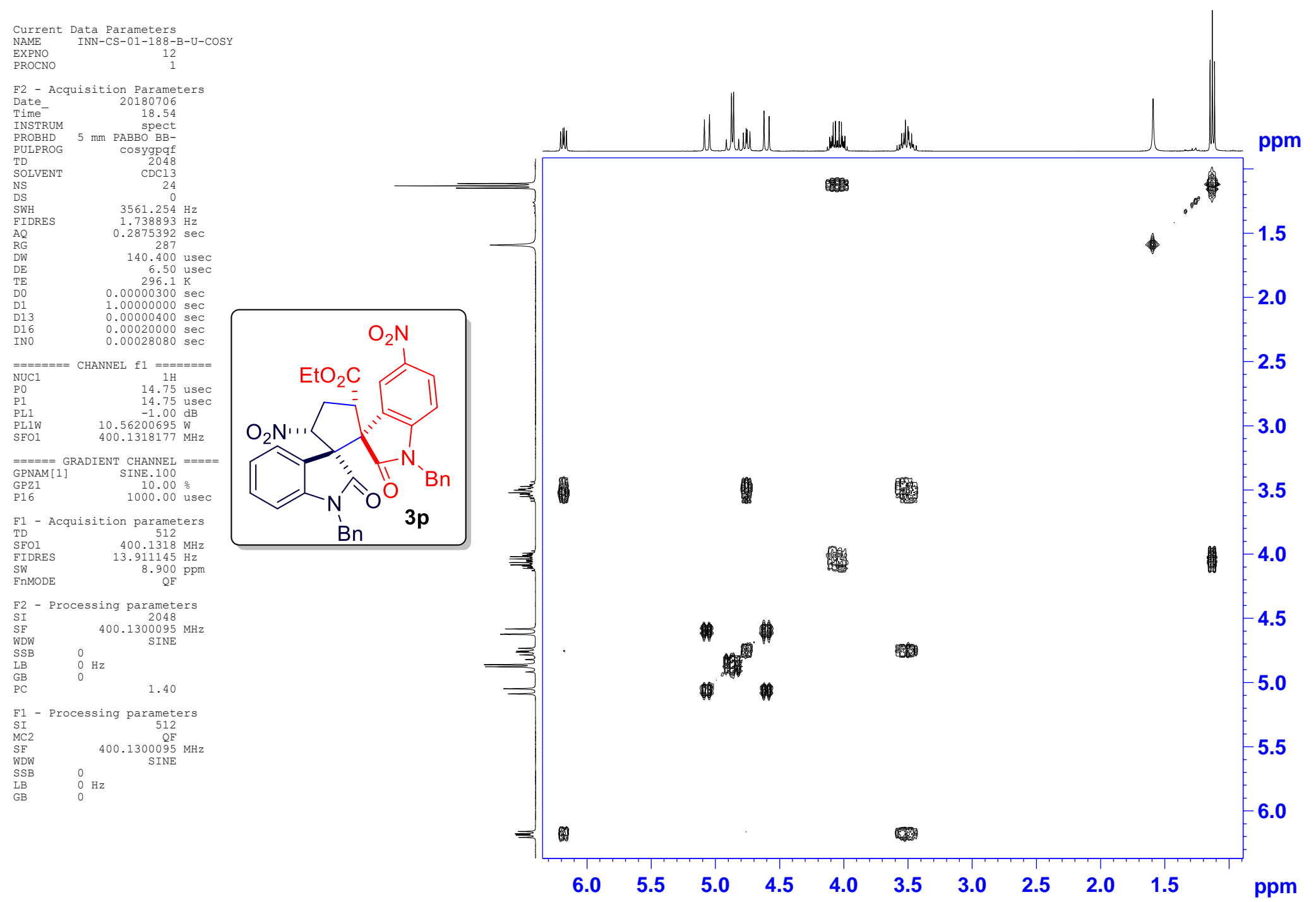

Figure S78. ${ }^{1} \mathrm{H}-{ }^{1} \mathrm{H}$ COSY NMR (400 MHz, $\mathrm{CDCl}_{3}$ ) Spectrum (Expansion) of $\mathbf{3 p}$ 


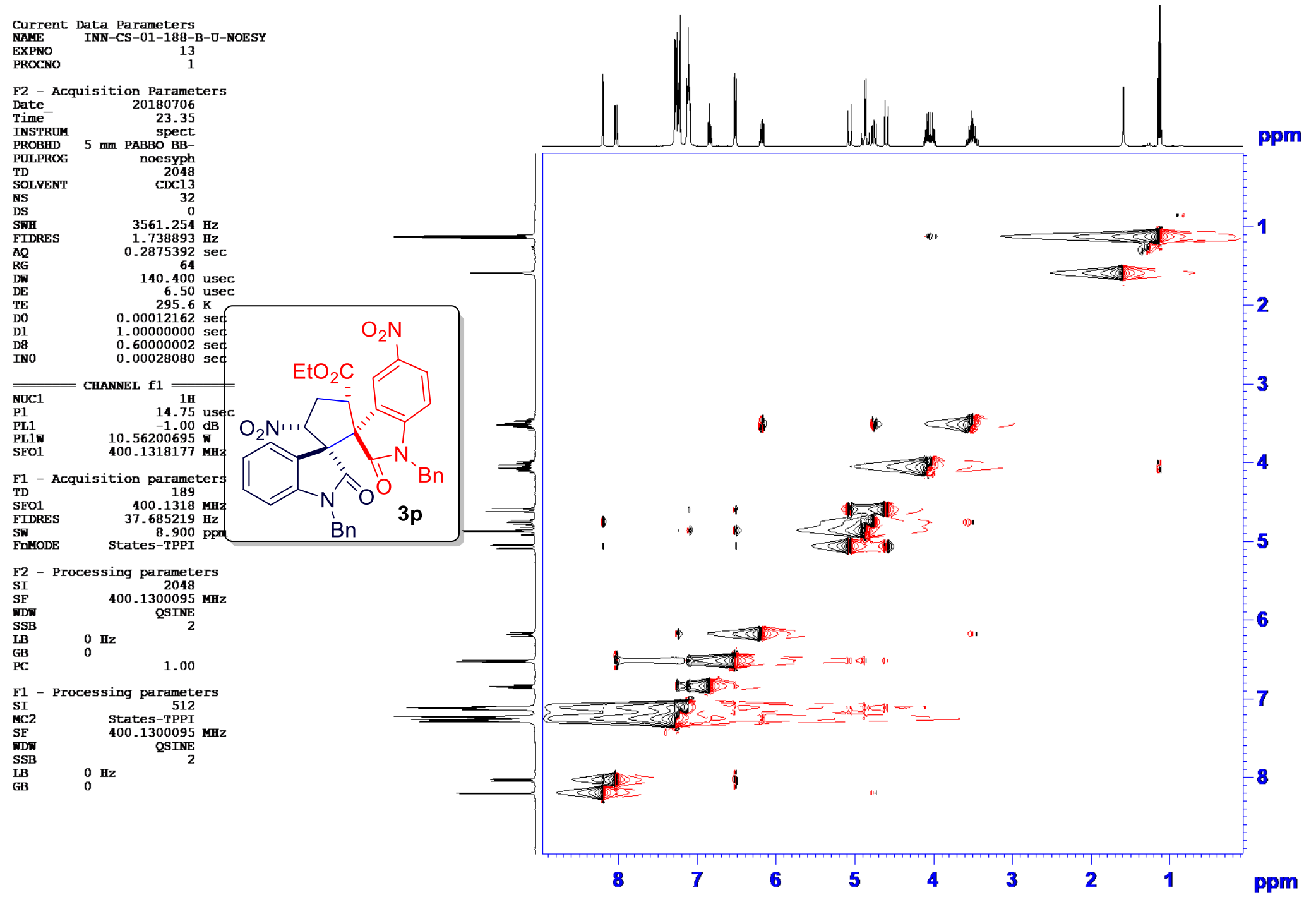

Figure S79. ${ }^{1} \mathrm{H}-{ }^{1} \mathrm{H}$ NOESY NMR (400 MHz, $\left.\mathrm{CDCl}_{3}\right)$ Spectrum of $\mathbf{3 p}$ 


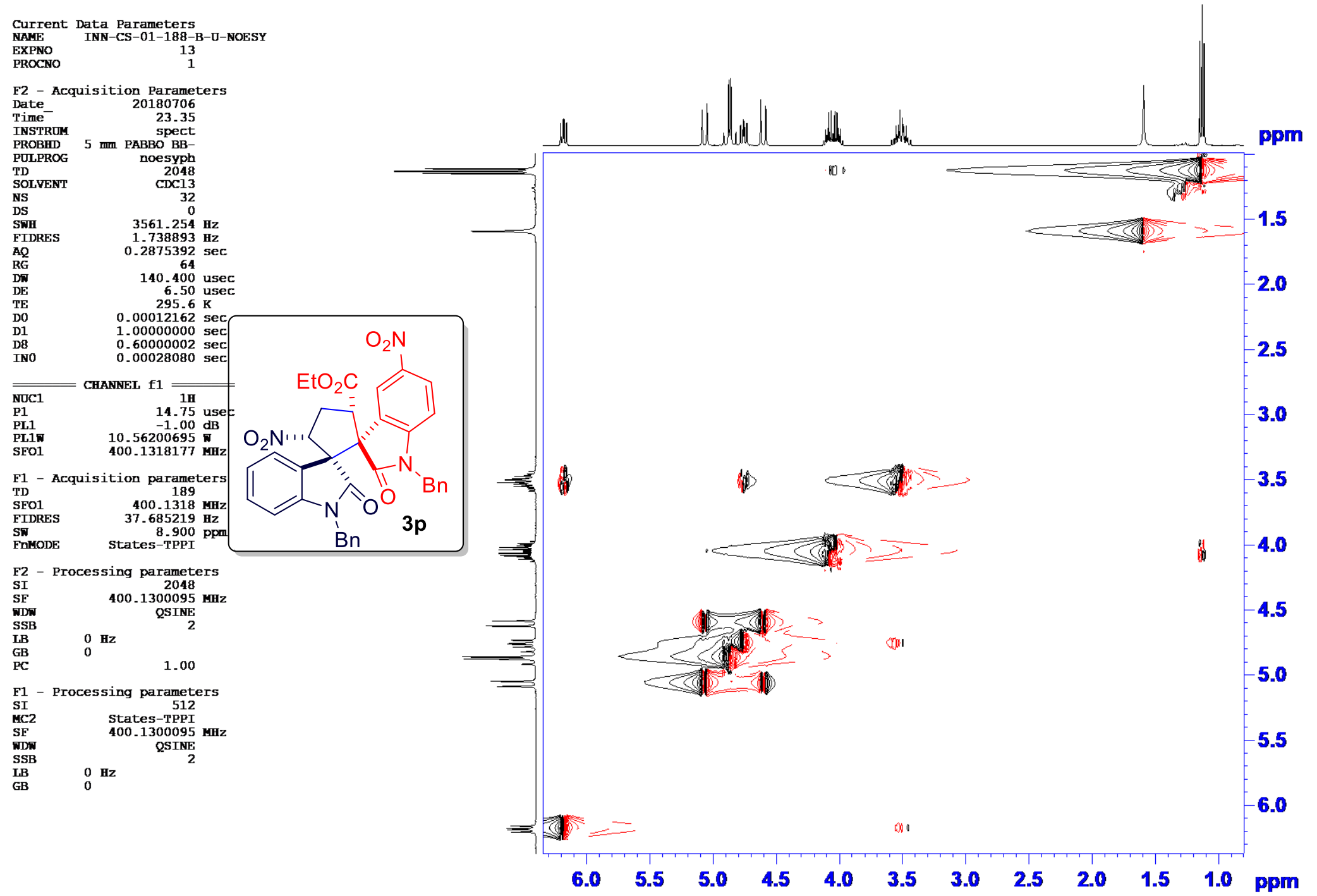

Figure S80. ${ }^{1} \mathrm{H}-{ }^{1} \mathrm{H}$ NOESY NMR (400 MHz, $\mathrm{CDCl}_{3}$ ) Spectrum (Expansion) of 3p 

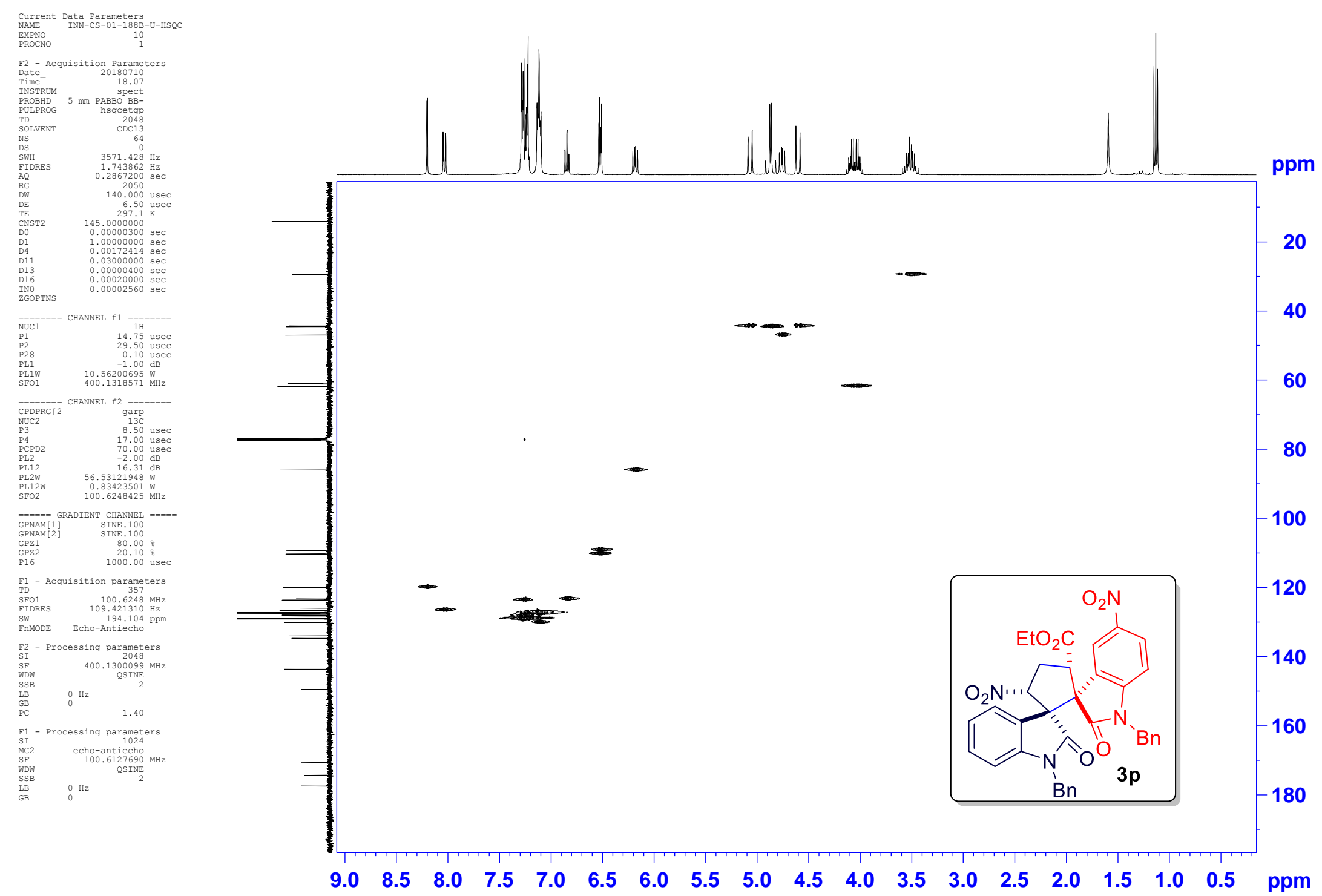

Figure S81. $1 \mathrm{H}-{ }^{13} \mathrm{C}$ HSQC NMR (100 MHz, $\left.\mathrm{CDCl}_{3}\right)$ Spectrum of $\mathbf{3 p}$ 

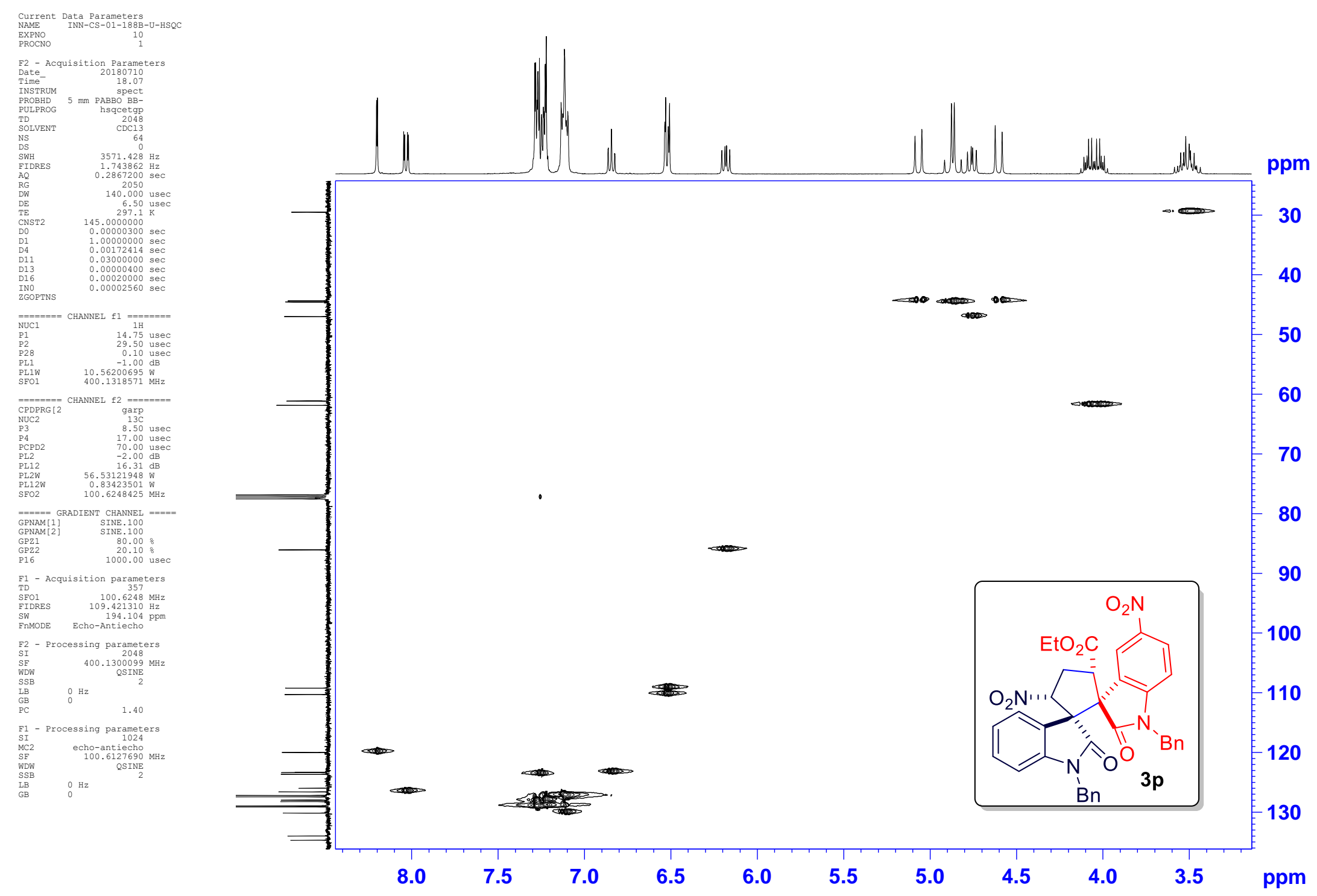

Figure S82. $1 \mathrm{H}-{ }^{13} \mathrm{C}$ HSQC NMR (100 MHz, $\mathrm{CDCl}_{3}$ ) Spectrum (Expansion) of $\mathbf{3 p}$ 


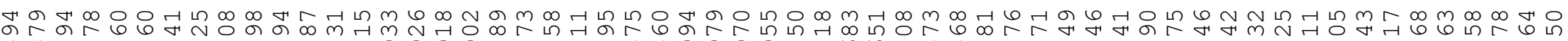

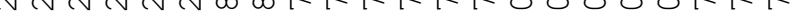

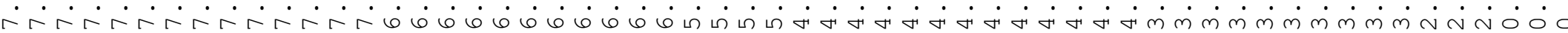

Current Data Parameters

NAME INN-CS-II-02-B-1H

PROCNO

F2 - Acquisition Parameters

Date_ 20171011

Time 18.25

PROBHD $5 \mathrm{~mm}$ PABBO BB/

PULPROG

TD

NS

zg30

SWH $\quad 10000.000 \mathrm{~Hz}$

FIDRES $\quad 0.152588 \mathrm{~Hz}$

AQ $\quad 3.2767999 \mathrm{sec}$

50.000 use

DE $\quad 6.50$ usec

D1 $\quad 1.00000000 \mathrm{sec}$

TDO

$=$ CHANNEL $f 1=======$

NUC1 13.35

P1 13.35 usec

PLW1

$16.00000000 \mathrm{~W}$

F2 - Processing parameters

SI $\quad 65536$

WDW

$\begin{array}{lll}\text { SSB } & 0 & \text { EM } \\ \text { SB } & 0 & \end{array}$

$\begin{array}{lll}\mathrm{LB} & 0 & 0.30 \\ \mathrm{~GB} & 1.00\end{array}$

1.00 N IN

7

6

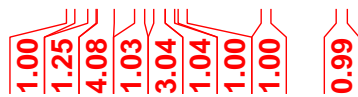

5

+) oty

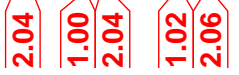

3

$\left|\begin{array}{l}3 \\ \vdots \\ \hdashline\end{array}\right|$

홍.

(b)

Figure S83. ${ }^{1} \mathrm{H} \mathrm{NMR}\left(500 \mathrm{MHz}, \mathrm{CDCl}_{3}\right)$ Spectrum of $\mathbf{3 q}$ 


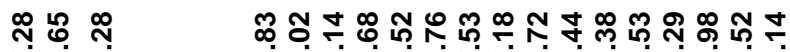

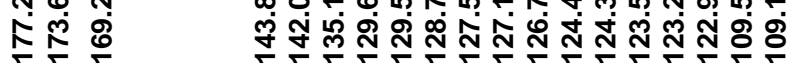

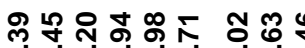

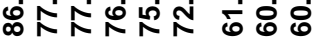

œ

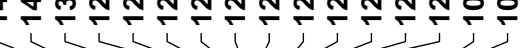

I/ V

Current Data Parameters
NAME
INN-CS-II-02-B-13C

NAME
EXPNO
PROCNO

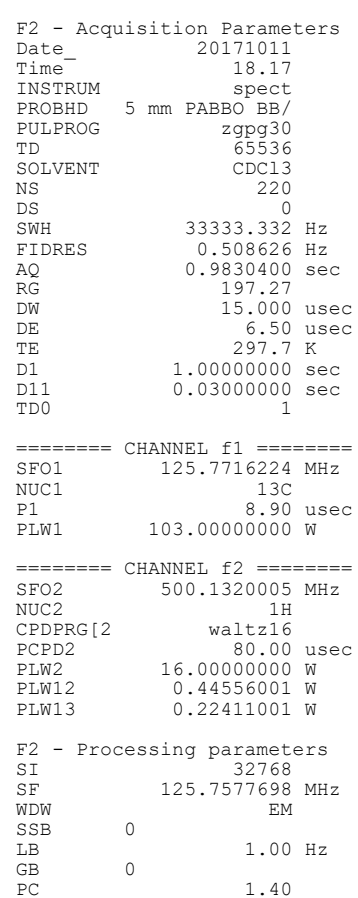

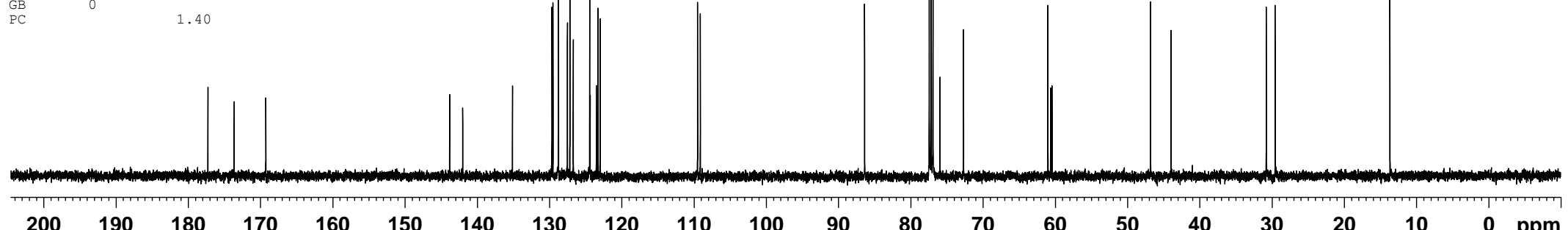

Figure S84. ${ }^{13} \mathrm{C}$ NMR (125 $\left.\mathrm{MHz}, \mathrm{CDCl}_{3}\right)$ Spectrum of $\mathbf{3 q}$ 


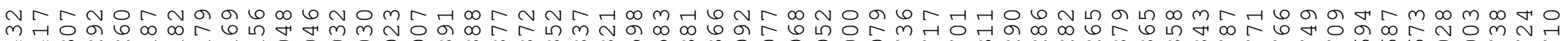
H $\mathrm{m} N \mathrm{~N}$ -

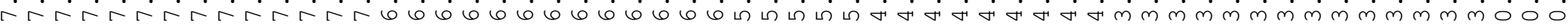

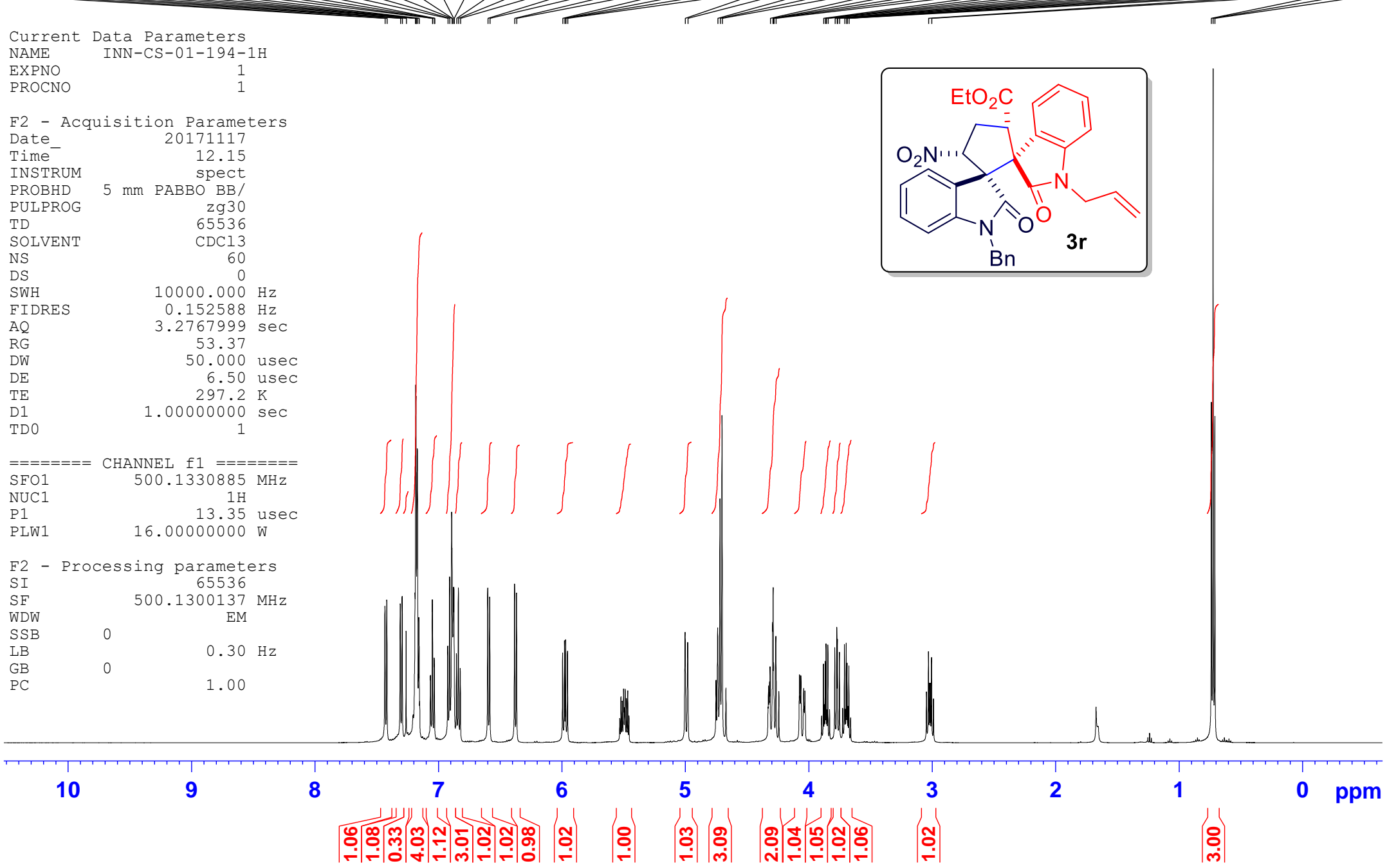

Figure S85. ${ }^{1} \mathrm{H}$ NMR $\left(500 \mathrm{MHz}, \mathrm{CDCl}_{3}\right)$ Spectrum of $\mathbf{3 r}$ 


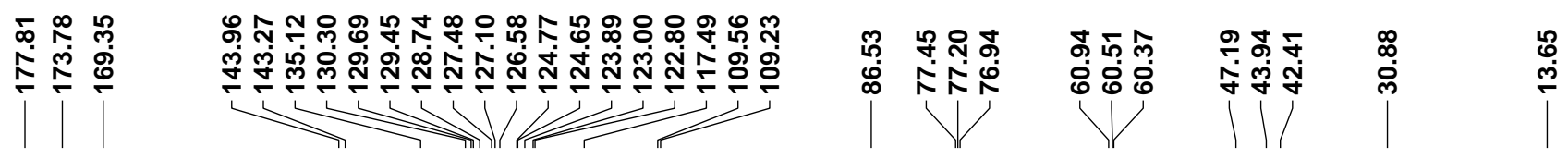

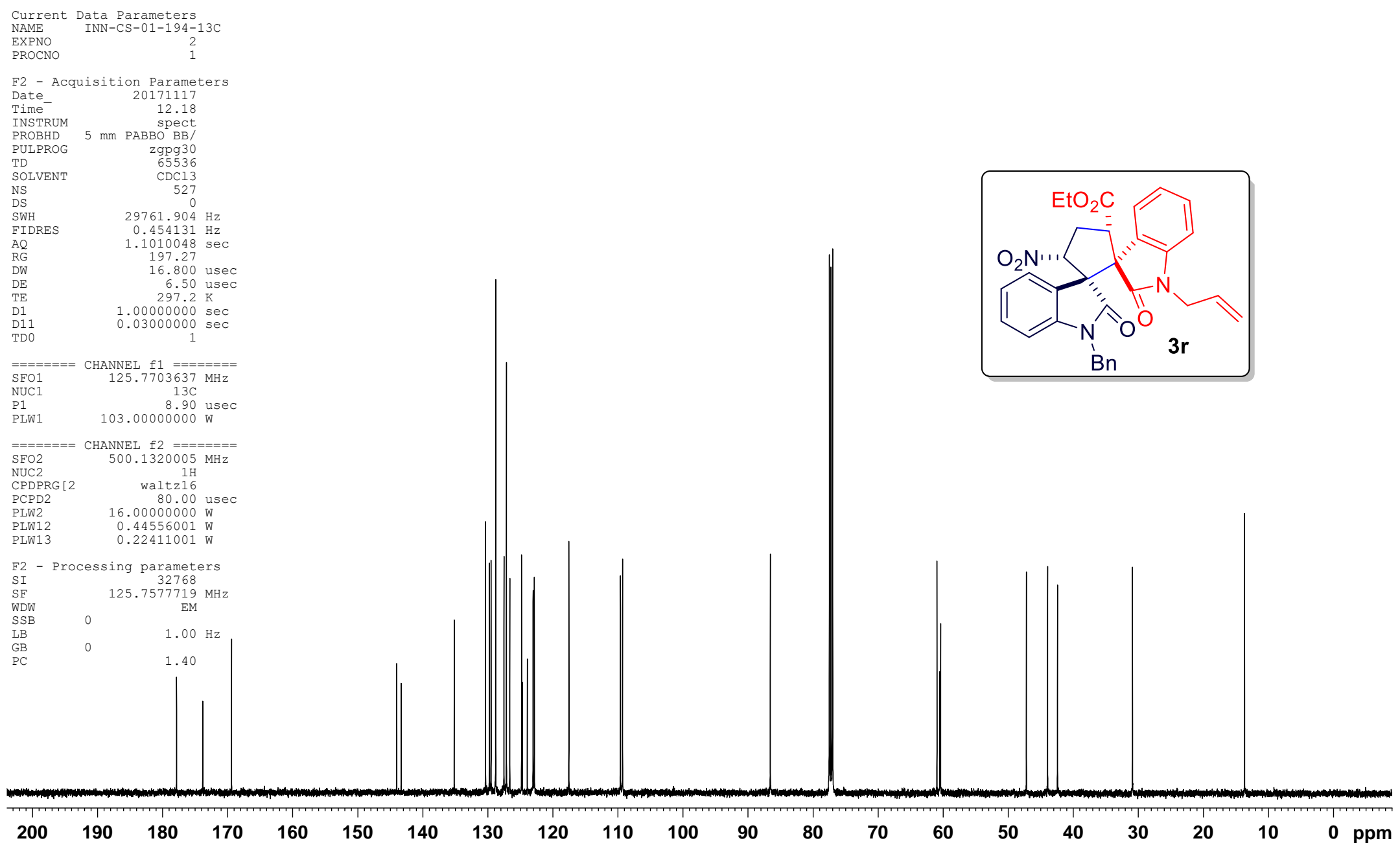

Figure S86. ${ }^{13} \mathrm{C}$ NMR (125 MHz, $\left.\mathrm{CDCl}_{3}\right)$ Spectrum of $\mathbf{3 r}$ 


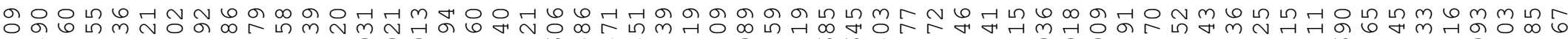

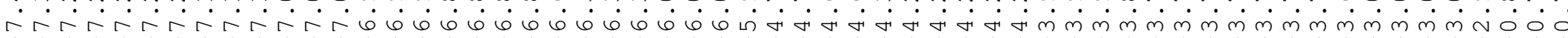
Current Data Parameters

NAME INN-CS-IV-164B-U-1H

EXPNO

ROCNO

F2 - Acquisition Parameters

Date__ 20210123

Time 18.00

SPECt
PROBHD $5 \mathrm{~mm}$ PABBO BB-

PULPROG $\quad$ zg30

TD

SOLV
NS

NS

SWH

FIDRES

RG

DE

TD0

$=======$ CHANNEL

NUC1

14.75 usec
$-1.00 \mathrm{~dB}$

$\begin{array}{ll}\text { PL1 } & -1.000 \mathrm{~dB}\end{array}$

SFO1 $400.1324710 \mathrm{MHz}$

F2 - Processing parameters

SI 32768

SF

SSB $\quad 0 \quad$ EM

$\begin{array}{ll}\mathrm{LB} & 0.30 \mathrm{~Hz}\end{array}$

1.00

1.00

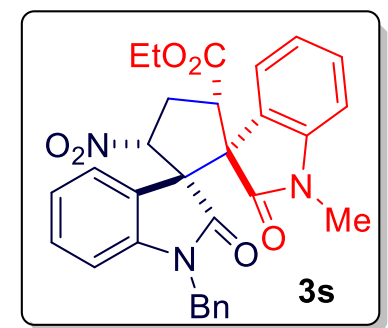

$\mathrm{Bn} 3 \mathrm{~s}$

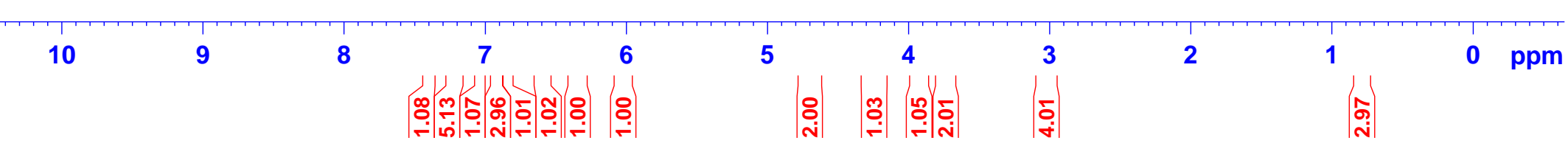

Figure S87. ${ }^{1} \mathrm{H}$ NMR (400 $\left.\mathrm{MHz}, \mathrm{CDCl}_{3}\right)$ Spectrum of $\mathbf{3 s}$ 


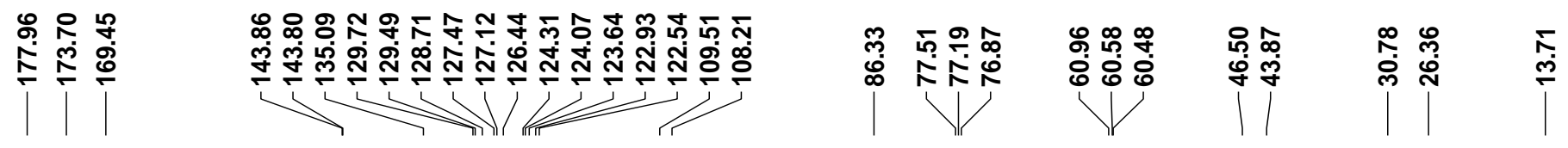

Current Data Parameters
NAME
EXPNO INN-CS-01-164B-U-1H
INPNO

EXPNO
PROCNO

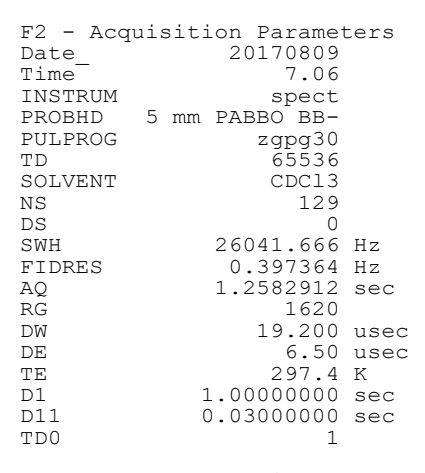

$\begin{array}{cr}===== & \text { CHANNEL } \mathrm{f} 1 \quad======= \\ 13 \mathrm{C} & 8.50 \mathrm{useC} \\ \mathrm{C} 1 & -2.00 \mathrm{~dB} \\ & 56.53121948 \mathrm{~W} \\ 1 \mathrm{~W} & 100.6238364 \mathrm{MHz}\end{array}$

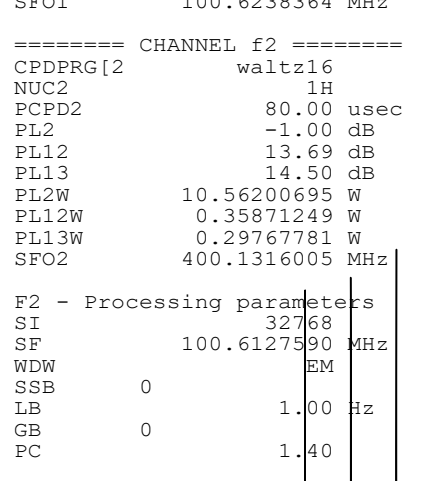

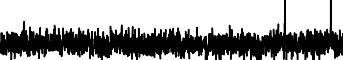

$\begin{array}{llll}200 & 190 & 180 & 170\end{array}$

170160

150

$140 \quad 130-120$

Figure S88. ${ }^{13} \mathrm{C}$ NMR $\left(100 \mathrm{MHz}, \mathrm{CDCl}_{3}\right)$ Spectrum of $\mathbf{3 s}$ 


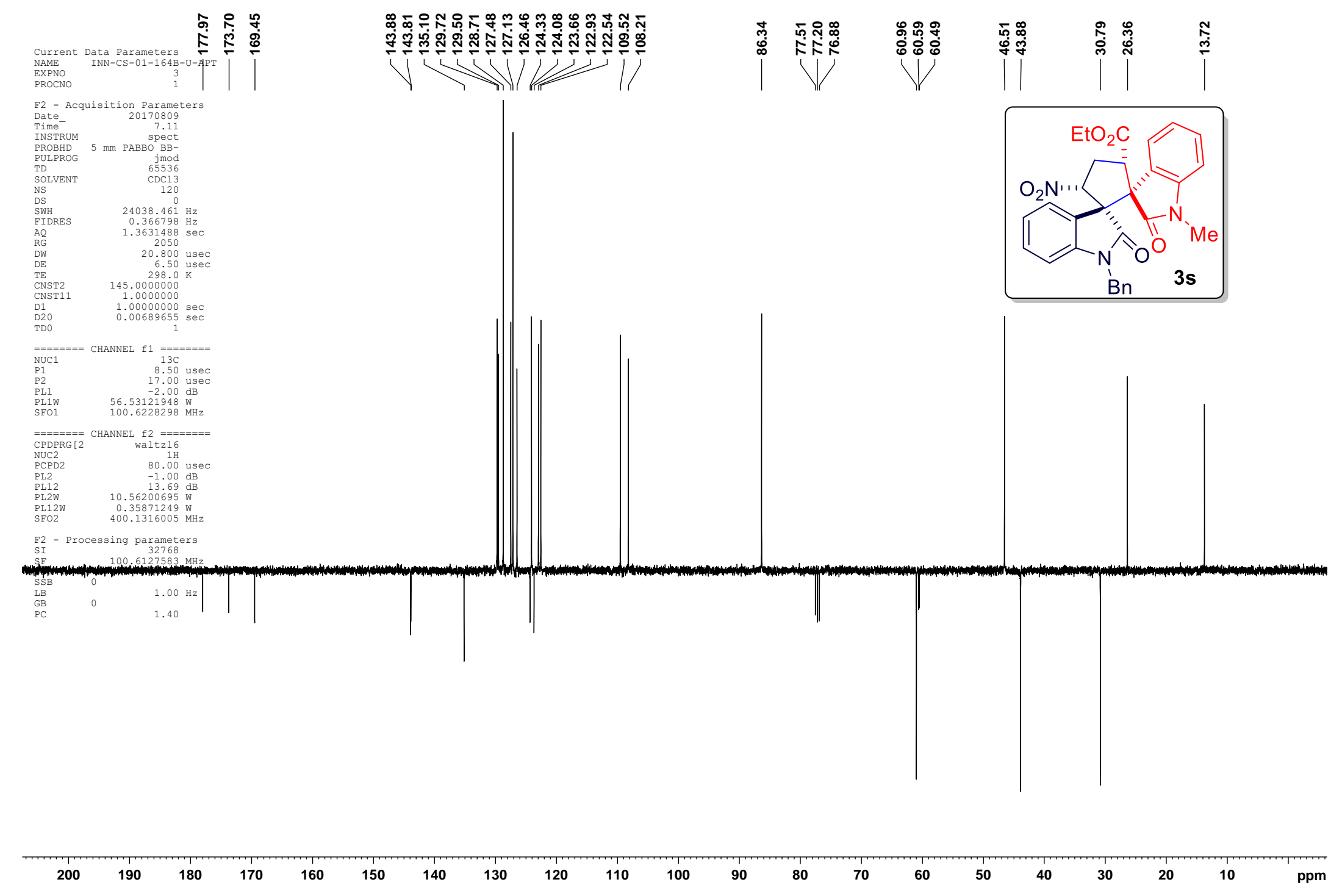

Figure S89. ${ }^{13} \mathrm{C}$-APT NMR $\left(100 \mathrm{MHz}, \mathrm{CDCl}_{3}\right)$ Spectrum of $\mathbf{3 s}$ 

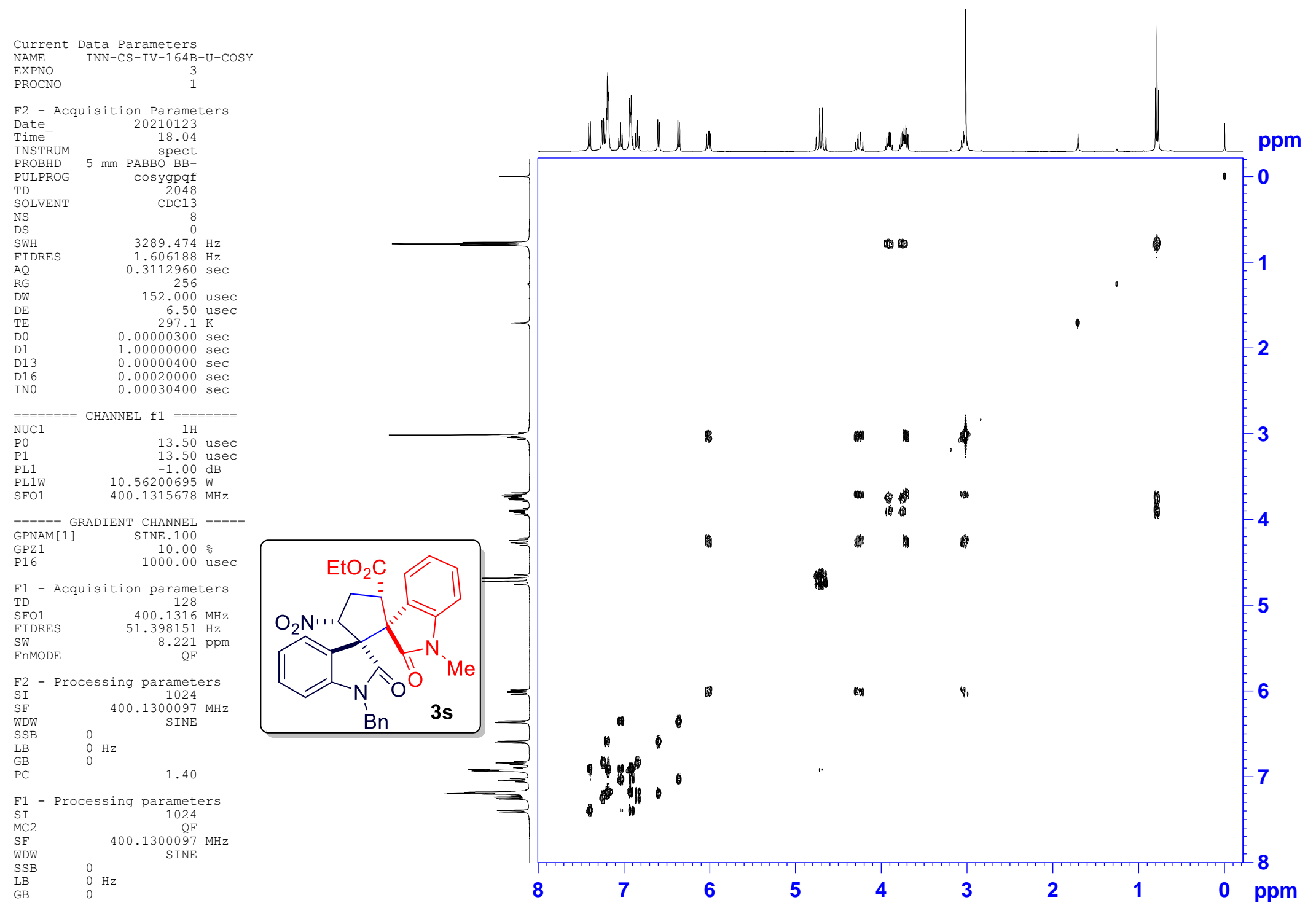

Figure S90. ${ }^{1} \mathrm{H}-\mathrm{H}$ COSY NMR $\left(400 \mathrm{MHz}, \mathrm{CDCl}_{3}\right)$ Spectrum of $\mathbf{3 s}$ 


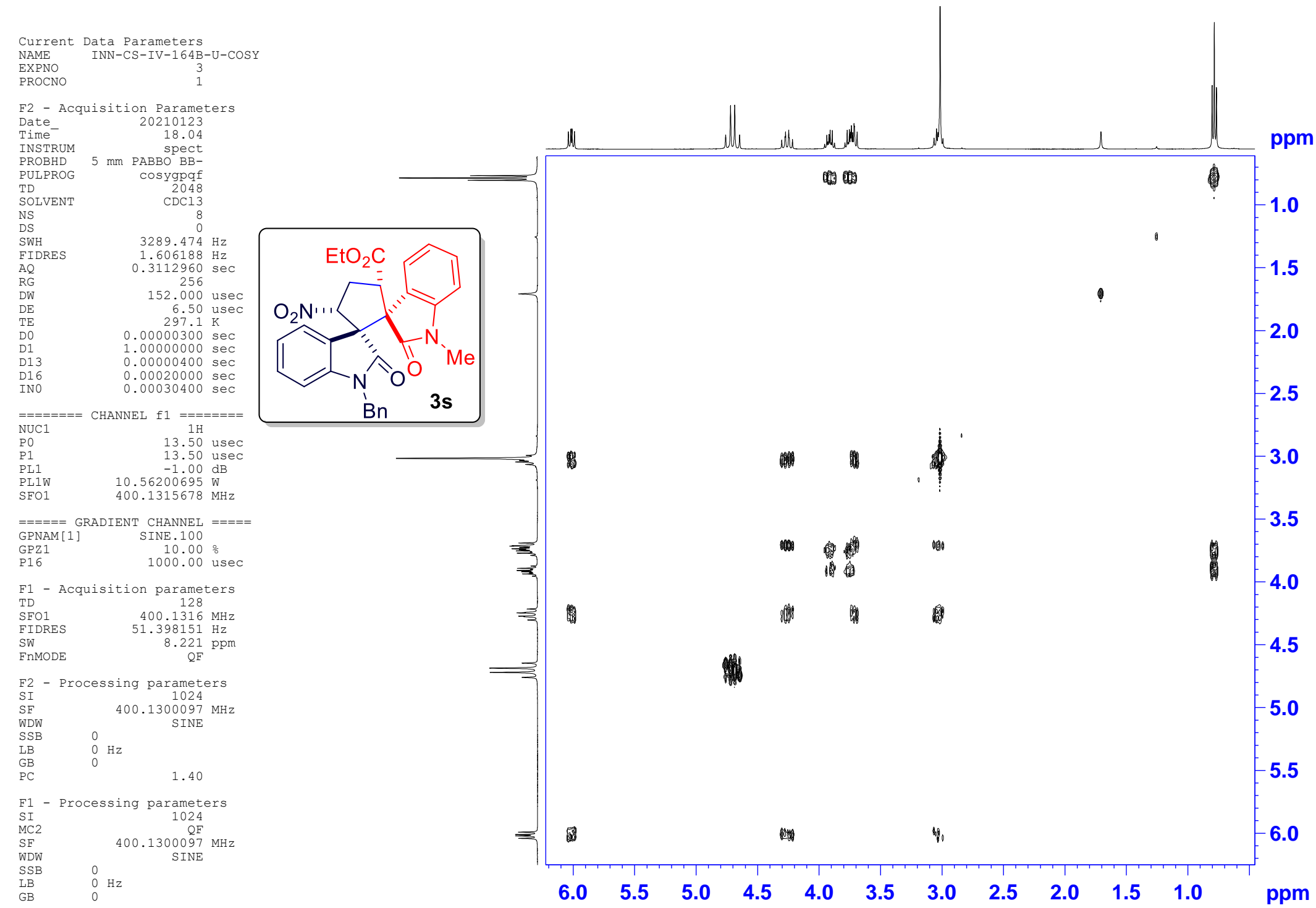

Figure S91. ${ }^{1} \mathrm{H}-\mathrm{H}$ COSY NMR ( $\left.400 \mathrm{MHz}, \mathrm{CDCl}_{3}\right)$ Spectrum (Expansion) of $\mathbf{3 s}$ 
Current
NAME INA-CS-IV-164B-U-COSY

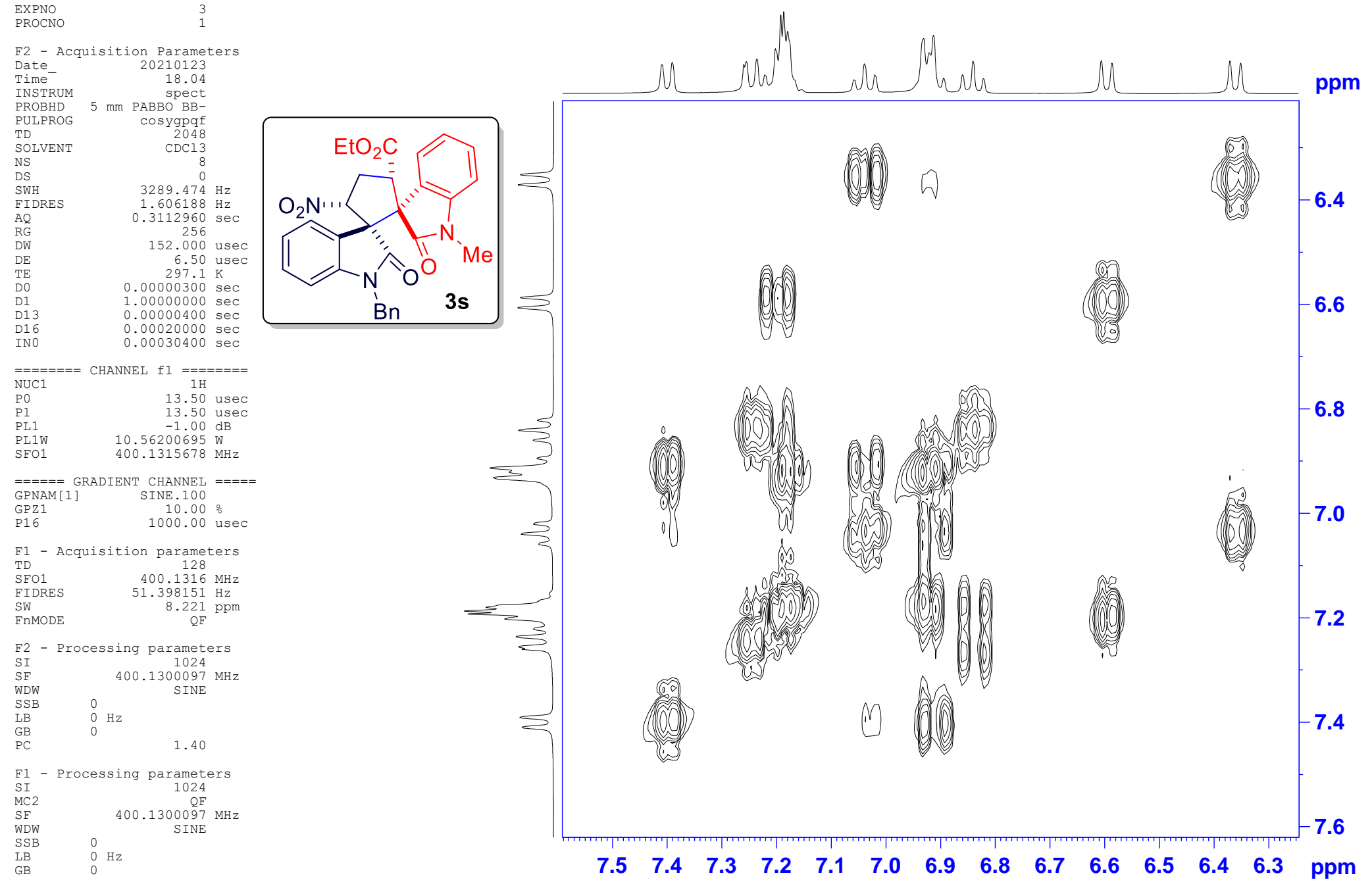

Figure S92. ${ }^{1} \mathrm{H}-\mathrm{H}$ COSY NMR (400 MHz, $\left.\mathrm{CDCl}_{3}\right)$ Spectrum (Expansion) of $\mathbf{3 s}$ 


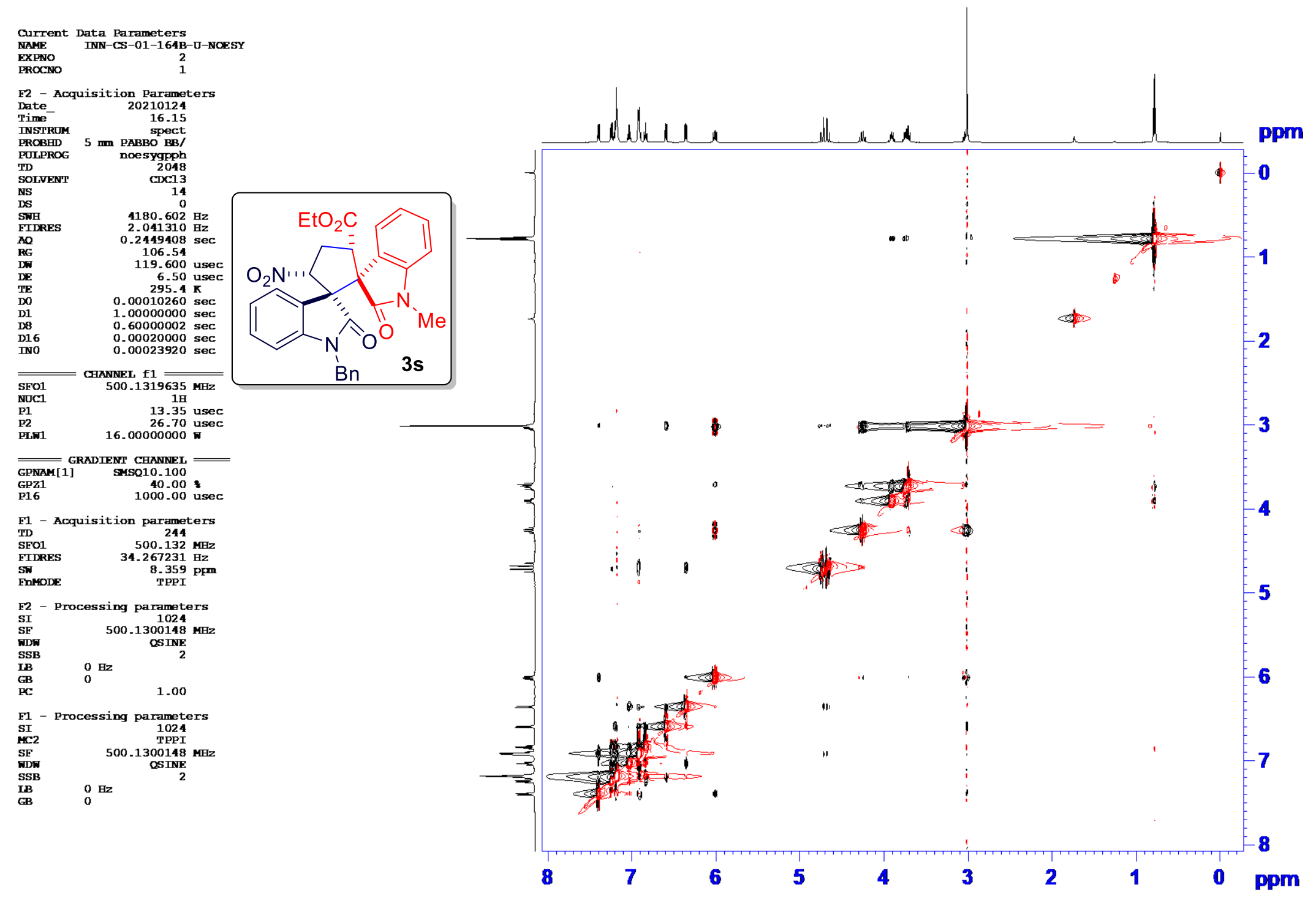

Figure S93. ${ }^{1} \mathrm{H}-\mathrm{H}$ NOESY NMR (500 MHz, $\left.\mathrm{CDCl}_{3}\right)$ Spectrum of $3 \mathbf{s}$ 


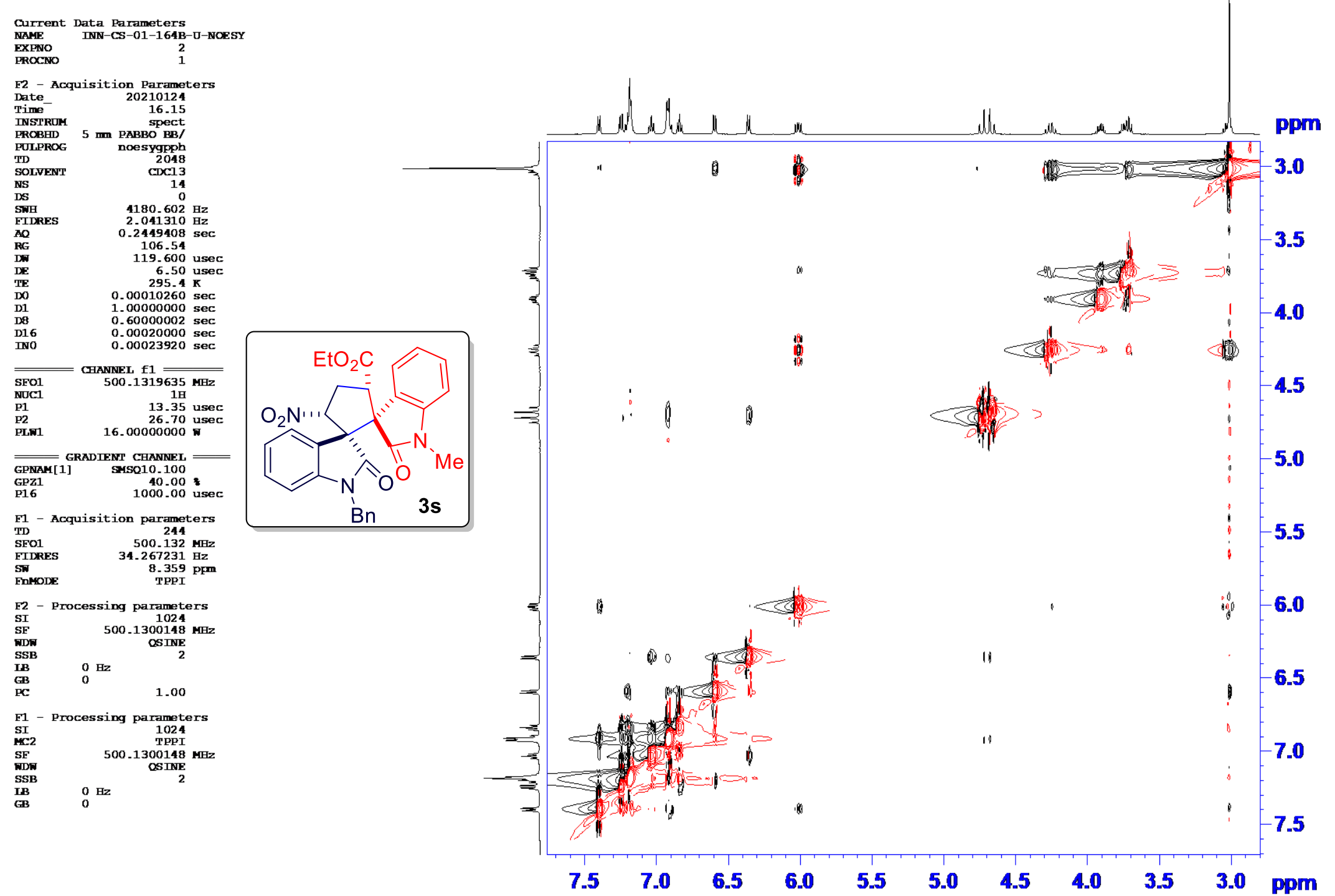

Figure S94. ${ }^{1} \mathrm{H}-\mathrm{H}$ NOESY NMR (500 MHz, $\mathrm{CDCl}_{3}$ ) Spectrum (Expansion) of $\mathbf{3 s}$ 


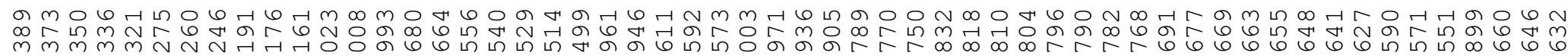
r.?.?.

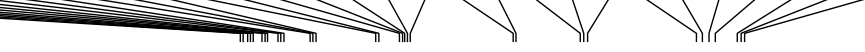

Current Data Parameters
NAME
INN-CS-01-164B-B-1H

PROCNO

F2 - Acquisition Parameters

Date__ 2018022

$\begin{array}{lr}\text { Time } & 9.36 \\ \text { INSTRUM } & \text { spect }\end{array}$

SPect
PROBHD $5 \mathrm{~mm}$ PABBO BB

PULPRO

TD

NS

$\begin{array}{lr}\text { NS } & 20 \\ \text { DS } & 0 \\ \text { SWH } & 10000.000 \mathrm{~Hz}\end{array}$

$0.152588 \mathrm{~Hz}$

$3.2767999 \mathrm{sec}$

$\begin{array}{ll}\text { DW } & 134.65 \\ \text { DW } & 50.000 \text { usec }\end{array}$

DE $\quad 6.50$ use

D1 $\quad 1.00000000 \mathrm{sec}$

TDO

$=======$ CHANNEL $\mathrm{fl}=======$

SFO1 $\quad 500.1330885 \mathrm{MHz}$

NUC1 $1 \mathrm{H}$

PLW1 $\quad 16.00000000 \mathrm{~W}$

F2 - Processing parameters

$\begin{array}{lr}\text { SI } & 65536 \\ \text { SF } & 500.1300135 \\ \text { MHz }\end{array}$

$\begin{array}{lr}\text { SF } & 500.1300135 \\ \text { WDW } & \text { EM }\end{array}$

$\begin{array}{lll}\text { SSB } & 0 & 0.30 \mathrm{~Hz}\end{array}$

$\begin{array}{lll}\mathrm{GB} & 0 & 1.00\end{array}$

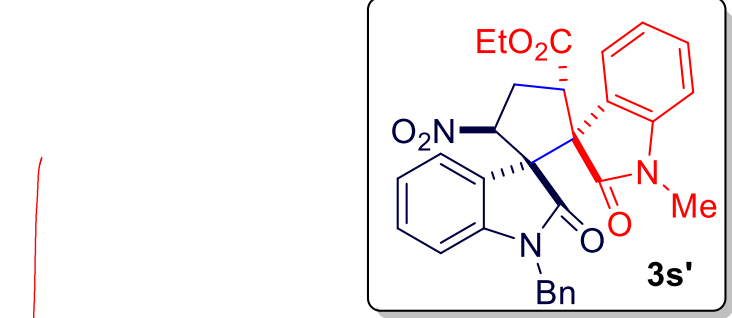

$\begin{array}{rrr}2 \\ 10 & 9 & 8\end{array}$

8

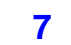

6

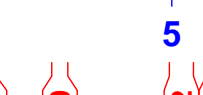

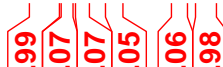

$\left(\begin{array}{l}\infty \\ 0 \\ 0\end{array}\right)$

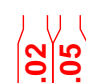

tas

+

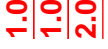

|ே.

Figure S95. ${ }^{1} \mathrm{H}$ NMR $\left(500 \mathrm{MHz}, \mathrm{CDCl}_{3}\right)$ Spectrum of 3s' 


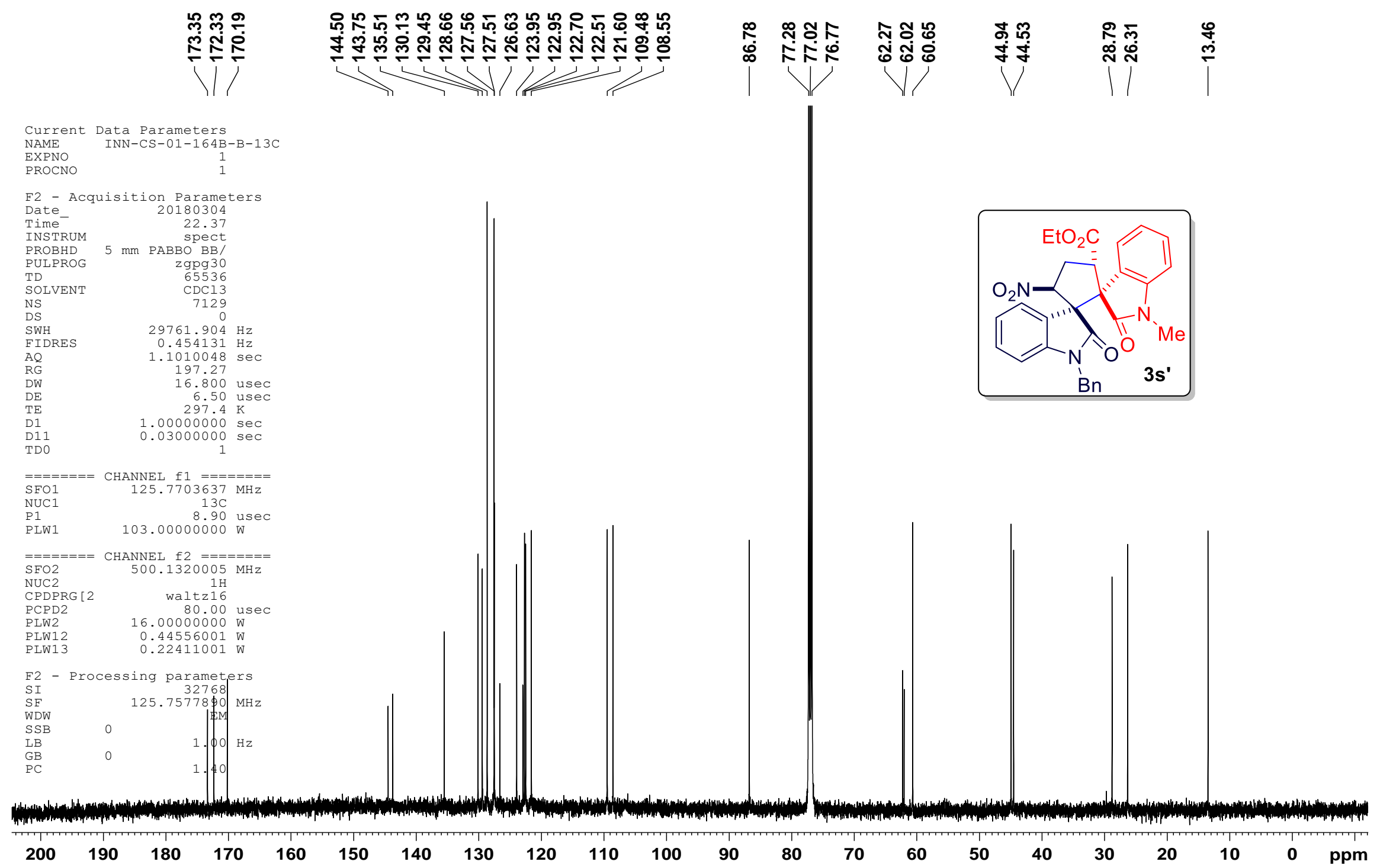

Figure S96. ${ }^{13} \mathrm{C} \mathrm{NMR}\left(125 \mathrm{MHz}, \mathrm{CDCl}_{3}\right)$ Spectrum of $\mathbf{3} \mathbf{s}^{\prime}$ 


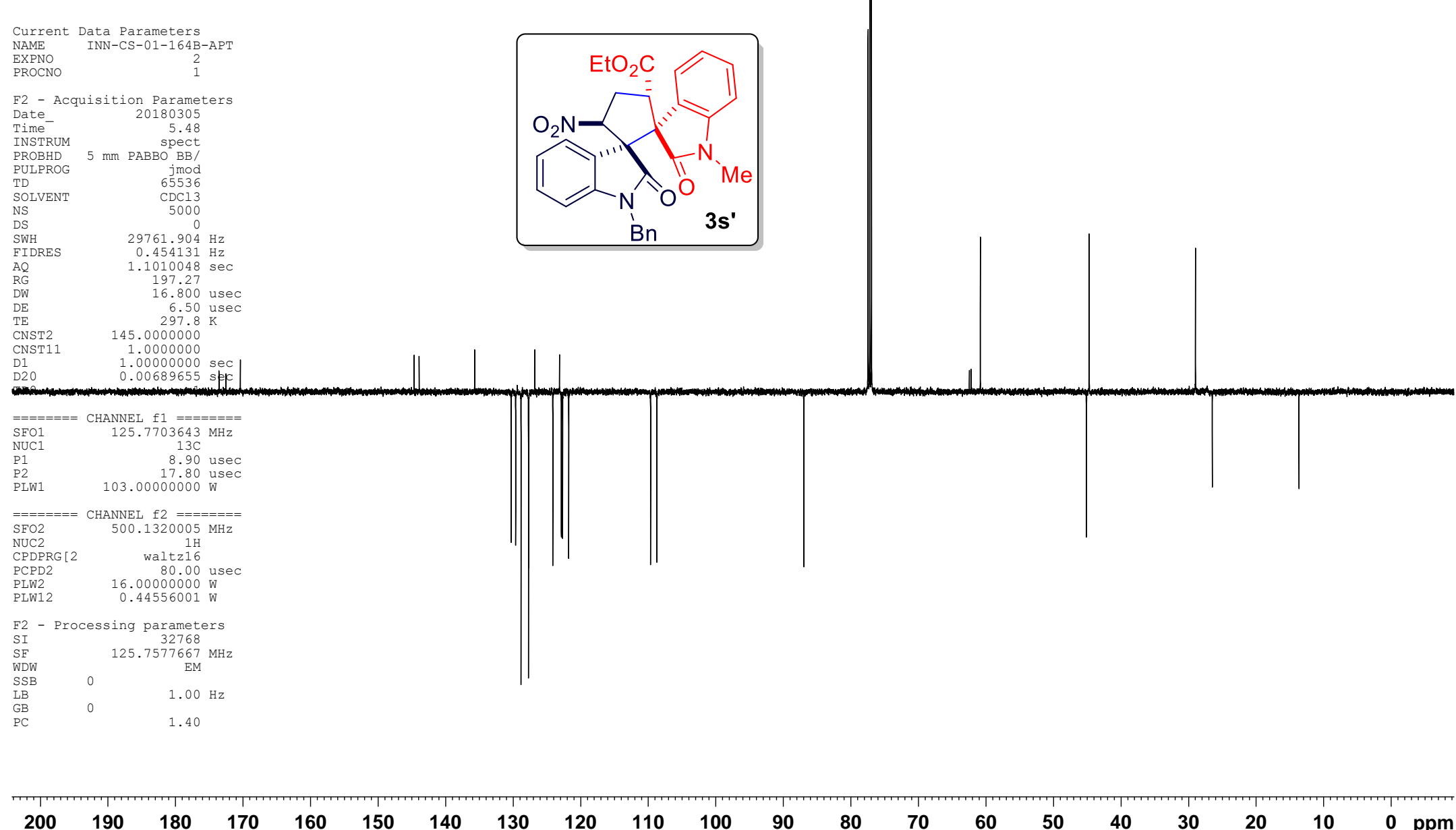

Figure S97. ${ }^{13} \mathrm{C}$-APT NMR (125 MHz, $\left.\mathrm{CDCl}_{3}\right)$ Spectrum of $3 \mathbf{s}^{\prime}$ 


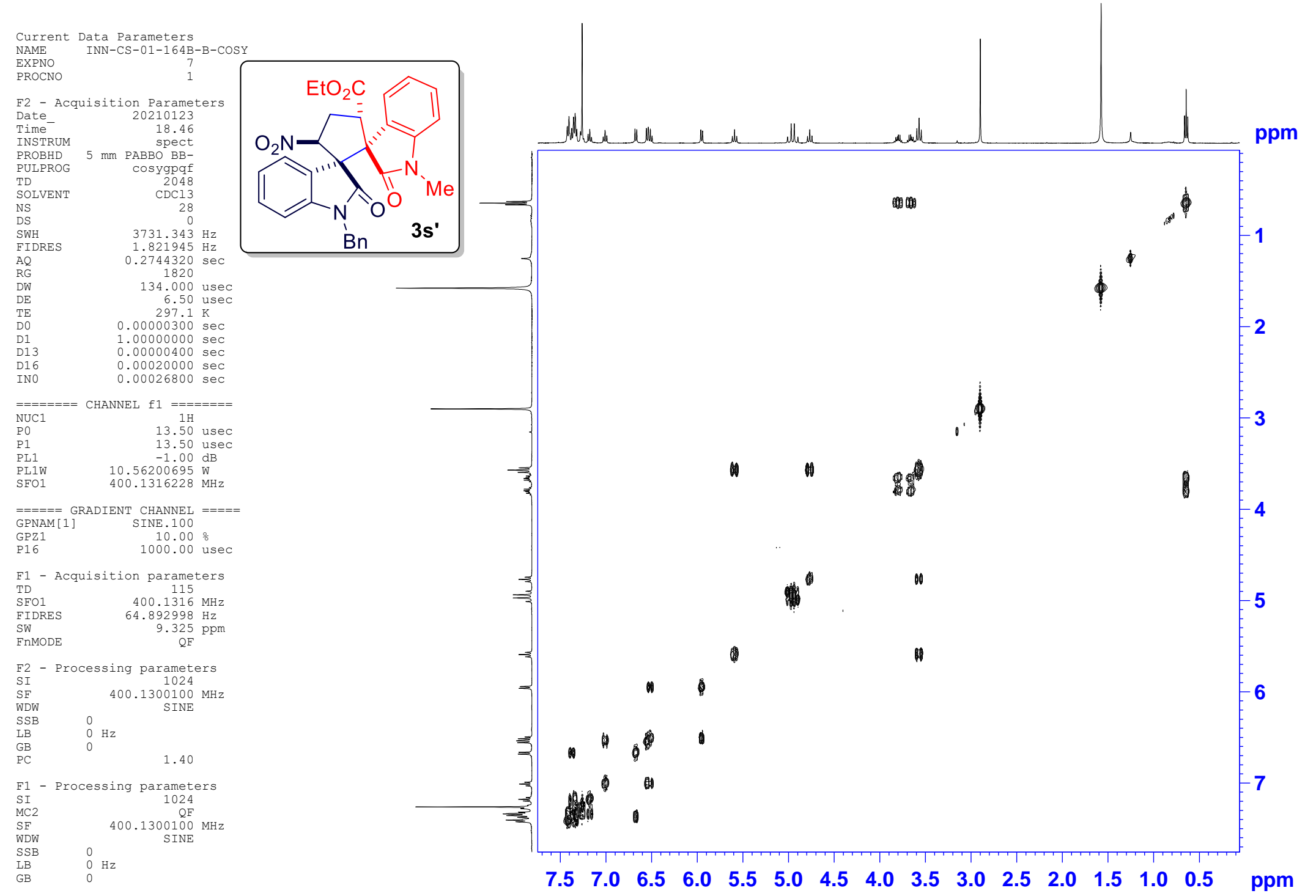

Figure S98. ${ }^{1} \mathrm{H}-\mathrm{H}$ COSY NMR $\left(400 \mathrm{MHz}, \mathrm{CDCl}_{3}\right)$ Spectrum of 3s' 


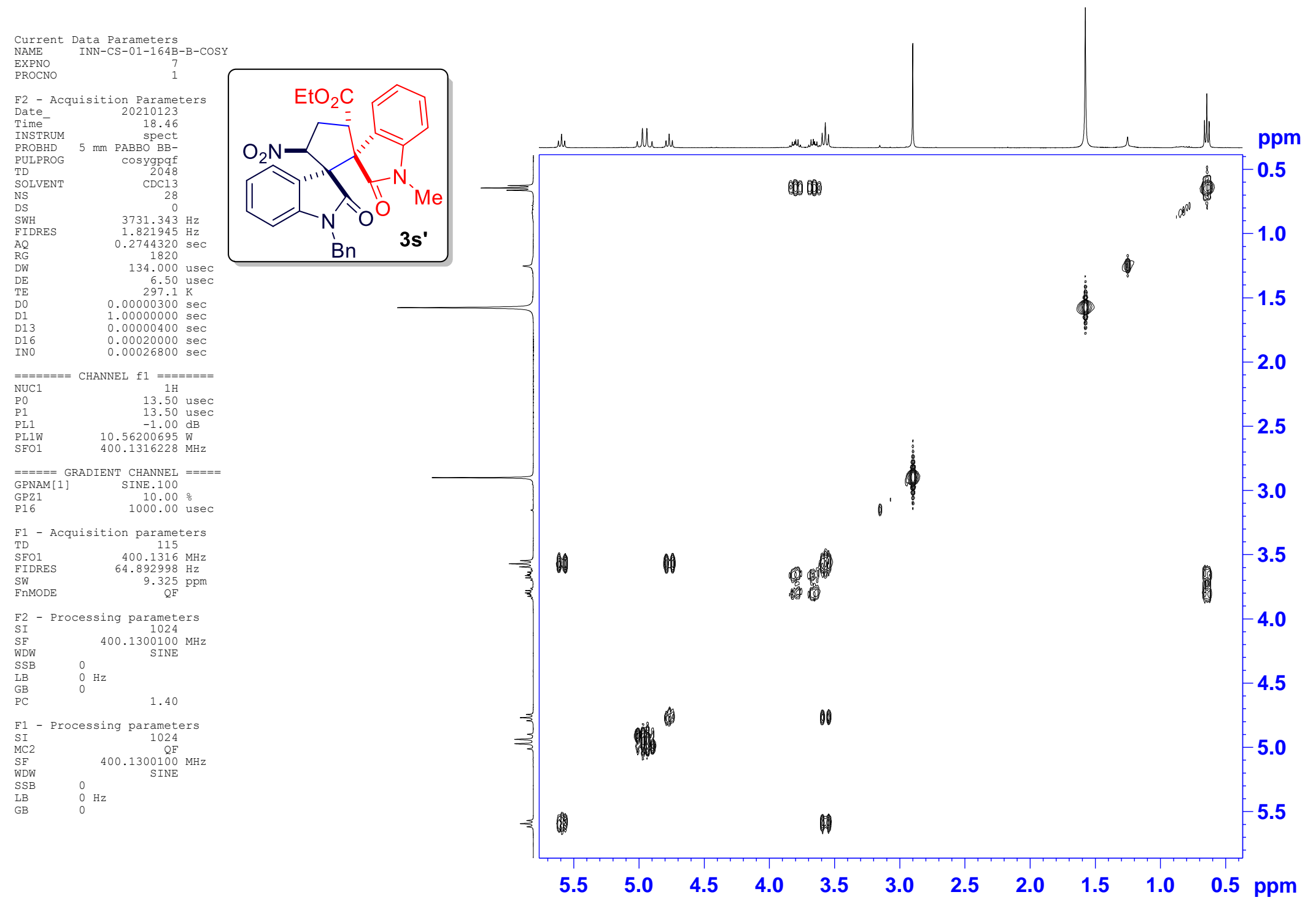

Figure S99. ${ }^{1} \mathrm{H}-\mathrm{H}$ COSY NMR (400 MHz, $\mathrm{CDCl}_{3}$ ) Spectrum (Expansion) of 3s' 


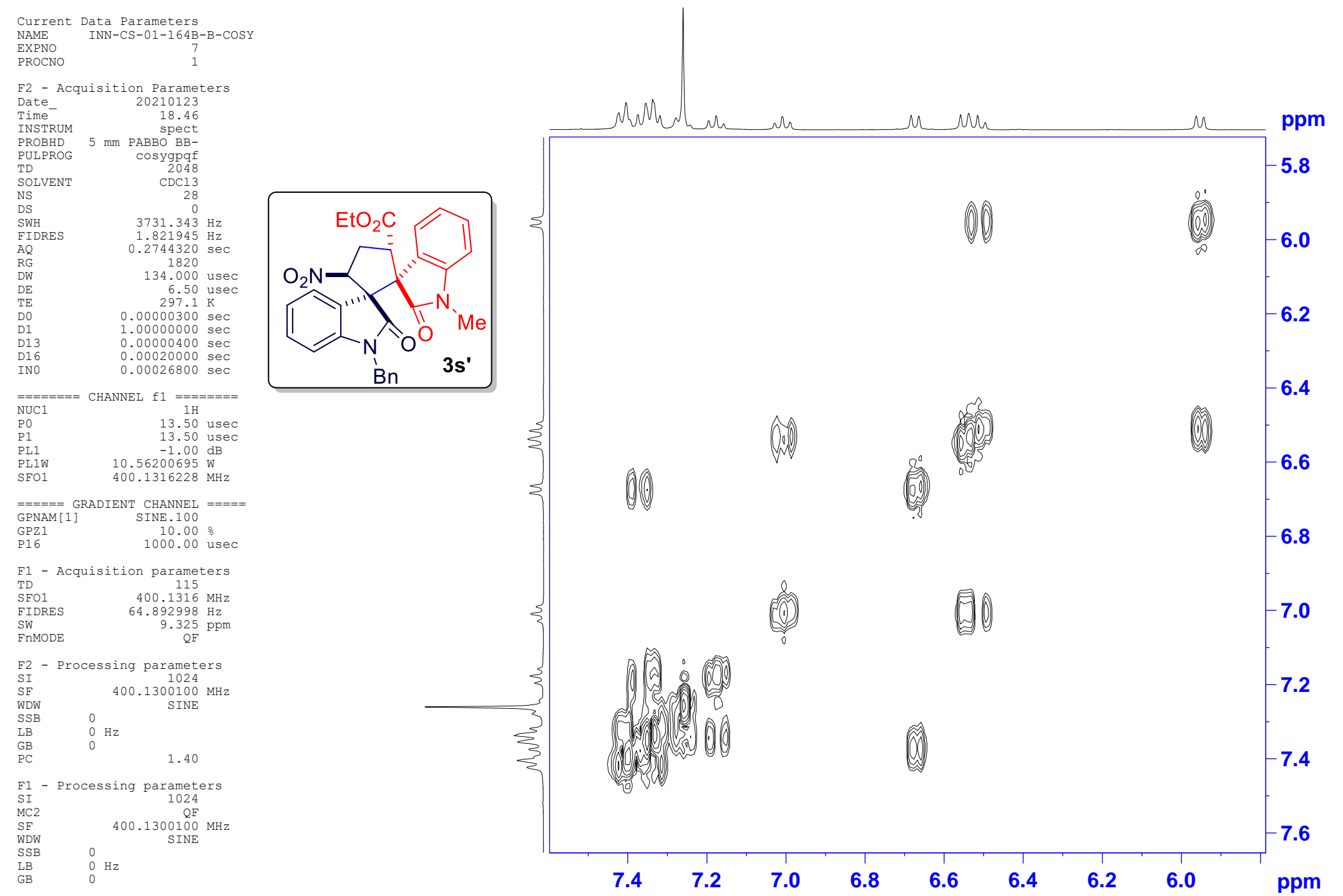

Figure S100. ${ }^{1} \mathrm{H}-\mathrm{H}$ COSY NMR (400 MHz, $\mathrm{CDCl}_{3}$ ) Spectrum (Expansion) of 3s' 
Current Data Parameters
NAME INN-CS-01-164B-B-NOESY EXPNO
PROCNO

F2 - Acquisition Parameters

Date_-

20210124
0.33

INSTRUM

DLLPROG 5 mm PABBO BB/

TD 2048

$\begin{array}{lr}\text { SOLVENT } & \mathrm{CDCl3} \\ \text { NS } & 10\end{array}$

$\begin{array}{ll}\text { DS } & 0 \\ \text { SWH } & \mathbf{4 4 2 4 . 7 7 9} \\ \text { FIDRES } & 2.160537 \text { HZ }\end{array}$

$\begin{array}{lr}\text { FIDRES } & 2.160537 \mathrm{~Hz} \\ \text { AQ } & 0.2314240 \mathrm{sec}\end{array}$

$\begin{array}{lr}\text { AQ } & 0.2314240 \mathrm{sec} \\ \text { RG } & 15.73 \\ \text { DW } & 113.000 \text { use }\end{array}$

$\begin{array}{lr}\mathrm{DE} & 6.50 \mathrm{use} \\ \mathrm{TE} & 295.4 \mathrm{~K}\end{array}$

$\begin{array}{ll}\text { D0 } & 0.00009600 \mathrm{sec} \\ \text { D1 } & 1.00000000 \mathrm{sec} \\ \text { D8 } & 0.60000002 \mathrm{sec}\end{array}$

$\begin{array}{ll}\text { D16 } & 0.00020000 \mathrm{sec} \\ \text { IN0 } & 0.00022600 \mathrm{sec}\end{array}$

$\overline{\overline{S F O 1}}=\underset{500.1319443 \mathrm{MHZ}}{\text { CHANEL } \mathrm{f1}}$

$\begin{array}{lr}\text { SFO1 } & 500.1319443 \mathrm{MHz} \\ \text { NUC1 } & 1 \mathrm{H} \\ \text { P1 } & 13.35 \text { usec } \\ \text { P2 } & 26.70 \text { usec } \\ \text { PLF1 } & 16.00000000 \text {. }\end{array}$

$\overline{\overline{\text { GPNAM }}}=\underset{\mathrm{G}}{\mathrm{G}} \mathrm{\text {GRADIENTCHANNEL }}$

$\begin{array}{lr}\text { GPNAM [1] } & \text { SHSQ10.100 } \\ \text { GPZ1 } & 40.00 \\ \text { P16 } & 1000.00 \text { use }\end{array}$

F1 - Acquisition parameters

$\begin{array}{lr}\text { T1 - Acquisition parameters } \\ \text { TD } & 1024 \\ \text { SFO1 } & 500.1319 \mathrm{MHz}\end{array}$

$\begin{array}{ll}\text { SFO1 } & 500.1319 \mathrm{MHz} \\ \text { FIDRES } & 8.642146 \mathrm{~Hz}\end{array}$

$\begin{array}{lc}\text { SW } & 8.847 \mathrm{ppm} \\ \text { FnHODE } & \text { TPPI }\end{array}$

F2 - Processing parameters

$\begin{array}{lc}\text { SI } & 1024 \\ \text { SF } & 500.1300151 \\ \text { NDW } & \text { QSINE }\end{array}$

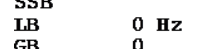

$\begin{array}{lll}\mathrm{GB} & 0 & 1.00\end{array}$

F1 - Processing parameters

$\begin{array}{ll}\text { SI } & 1024 \\ \text { MC2 } & \text { TPPI }\end{array}$

$\begin{array}{lc}\text { HC2 } & \text { TPPI } \\ \text { SF } & 500.1300151 \text { MHZ } \\ \text { WDW } & \text { QSINE }\end{array}$

$\begin{array}{lll}\text { LB } & 0 & \text { Hz } \\ \text { GB } & 0\end{array}$

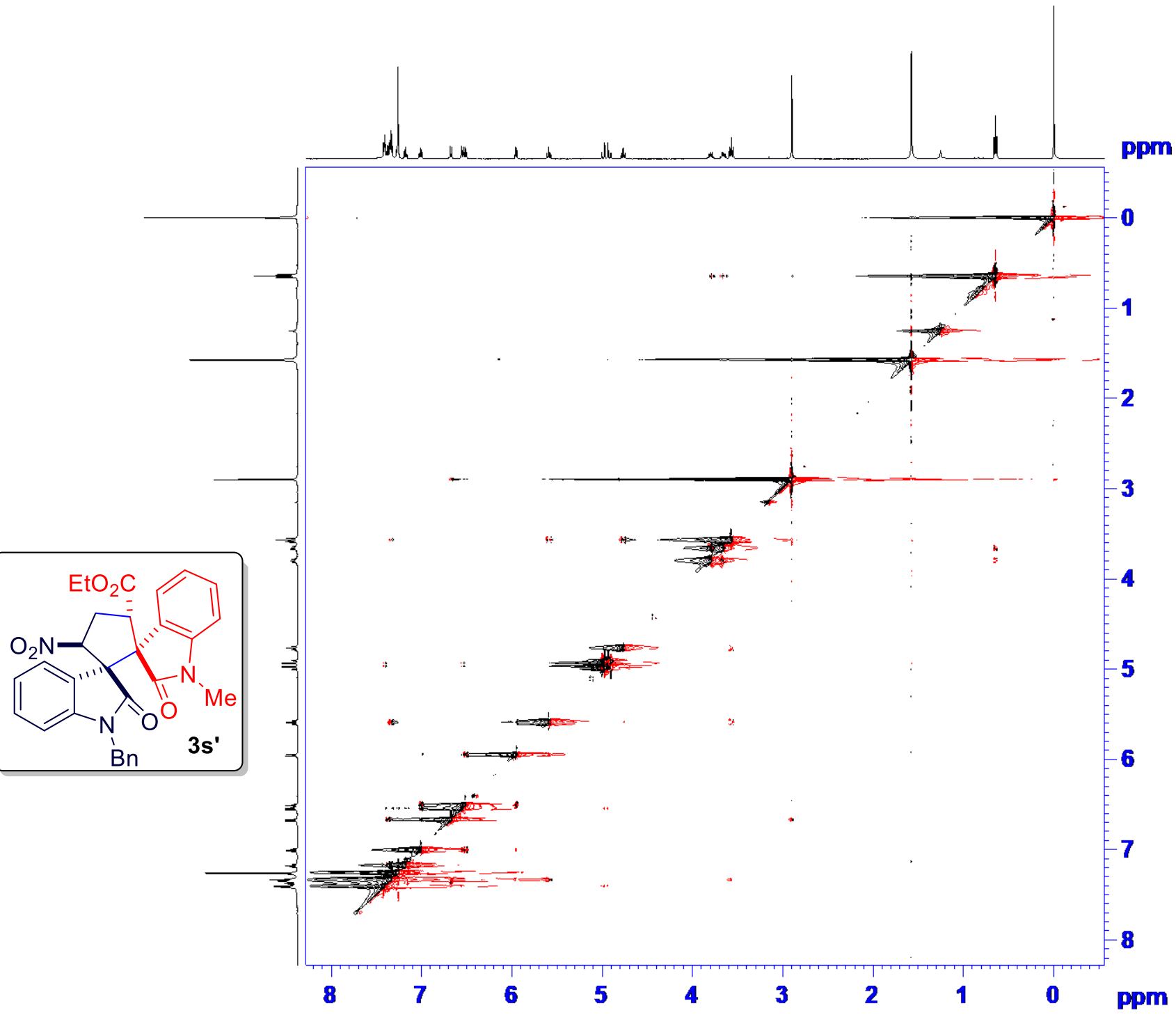

Figure S101. ${ }^{1} \mathrm{H}-\mathrm{H}$ NOESY NMR $\left(500 \mathrm{MHz}, \mathrm{CDCl}_{3}\right)$ Spectrum of $\mathbf{3 s}$ ' 
Current Data Parameters
NAME INN-CS-01-164B-B-NOESY EXXNO
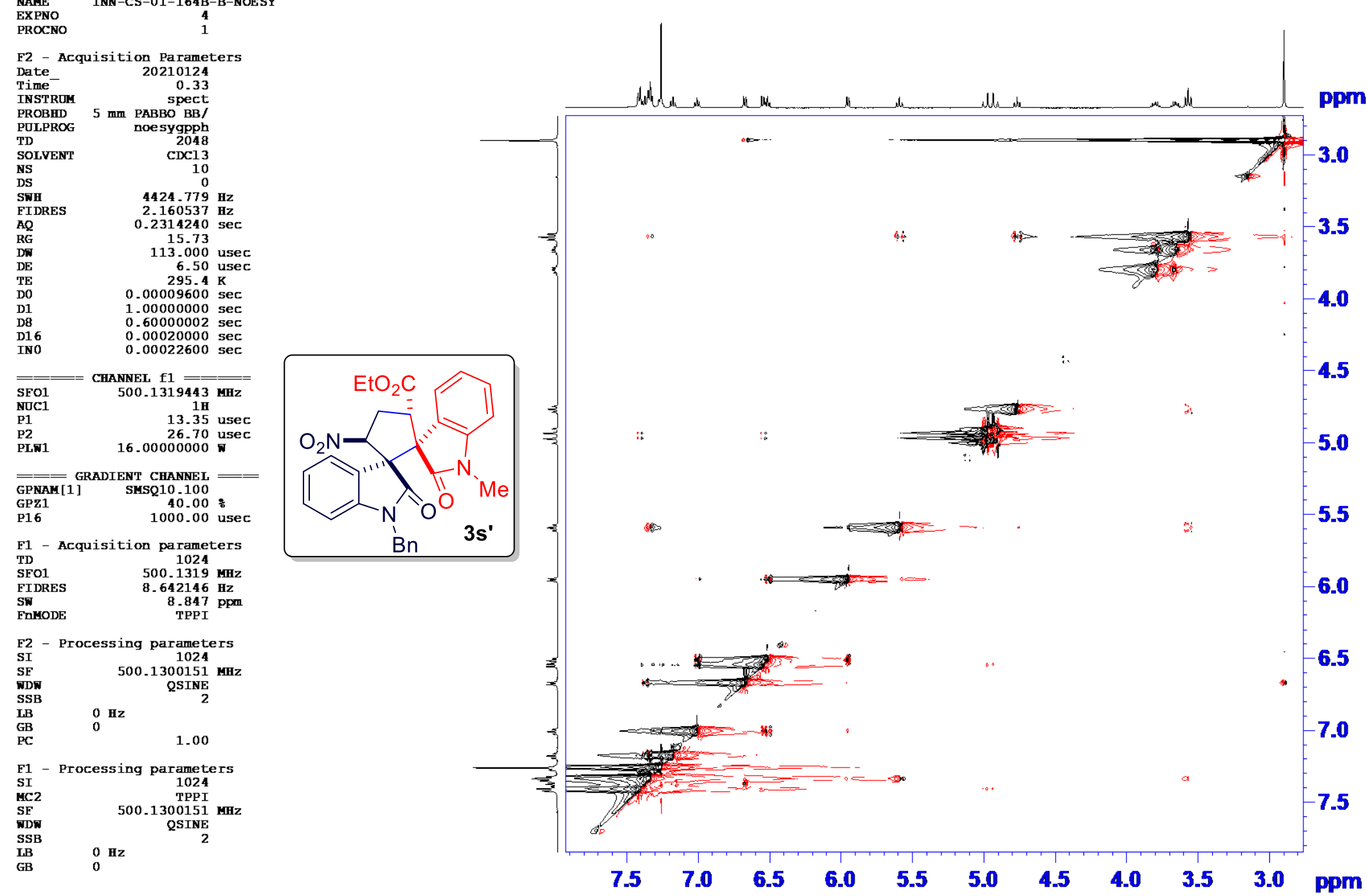

Figure S102. ${ }^{1} \mathrm{H}-\mathrm{H}$ NOESY NMR $\left(500 \mathrm{MHz}, \mathrm{CDCl}_{3}\right)$ Spectrum (Expansion) of 3s' 


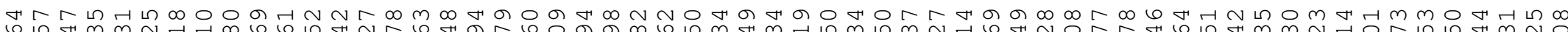

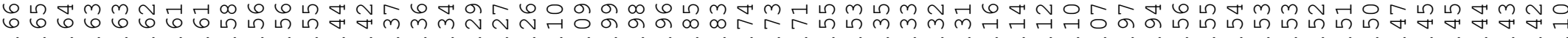
r

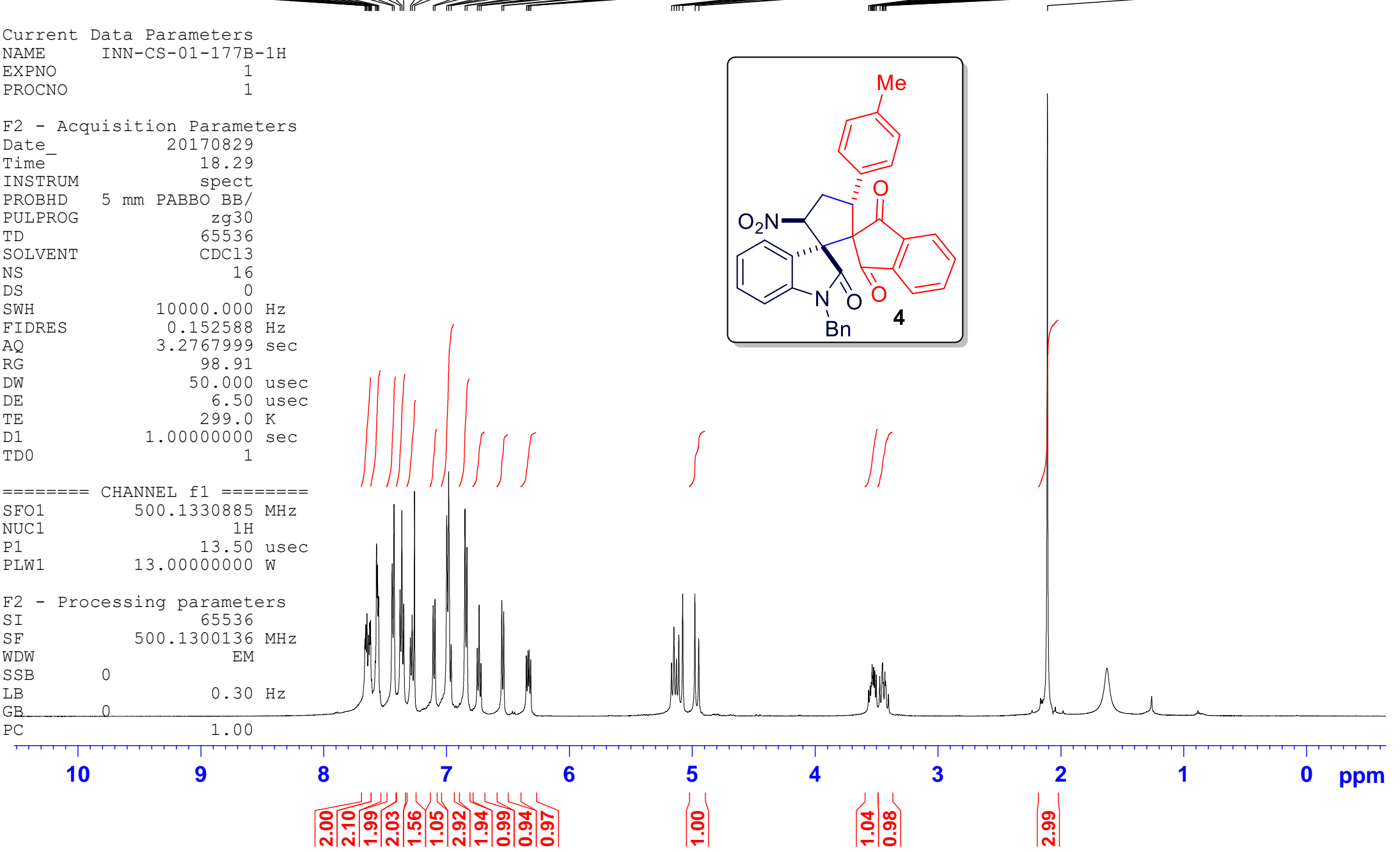

Figure S103. ${ }^{1} \mathrm{H} \mathrm{NMR}\left(500 \mathrm{MHz}, \mathrm{CDCl}_{3}\right)$ Spectrum of 4 


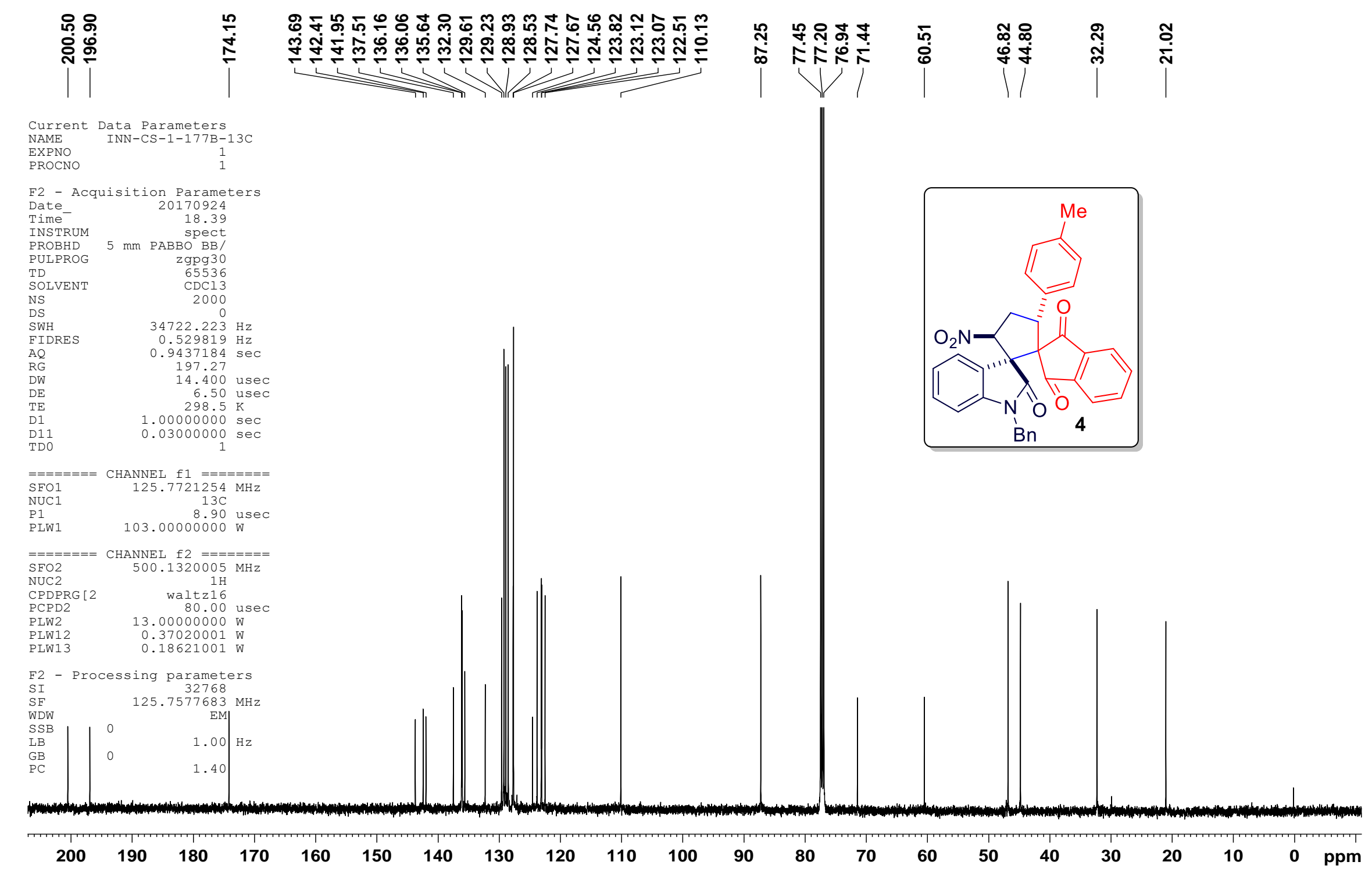

Figure S104. ${ }^{13} \mathrm{C}$ NMR (125 MHz, $\left.\mathrm{CDCl}_{3}\right)$ Spectrum of 4 

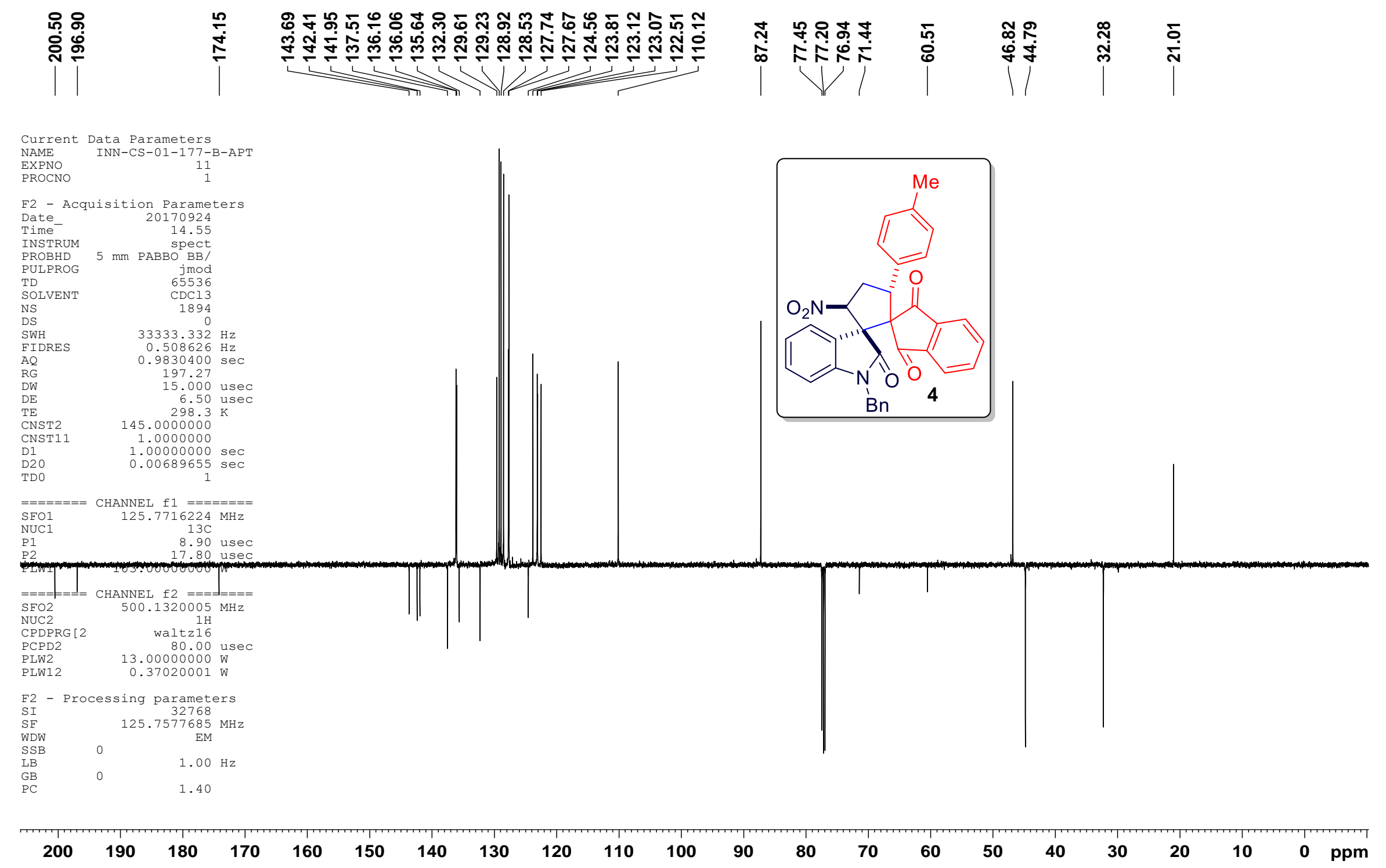

Figure S105. ${ }^{13} \mathrm{C}$-APT NMR (125 MHz, $\left.\mathrm{CDCl}_{3}\right)$ Spectrum of 4 


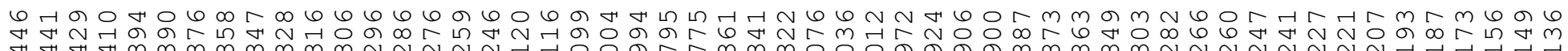

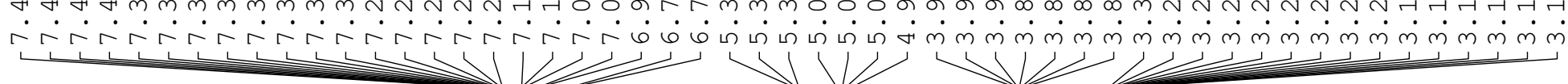

Current Data Parameters

INN-CS-II-26-1H

NAME

PROCNO

F2 - Acquisition Parameters

20180713
11.02

$\begin{array}{ll}\text { Time } & 11.02 \\ \text { INSTRUM } & \text { spect }\end{array}$

PROBHD $5 \mathrm{~mm}$ PABBO BB-

PROBHD

TD

SOL

DS

$\mathrm{CDCl}$
25

SWH $\quad 8223.685 \mathrm{~Hz}$

FIDRES $\quad 0.151522 \mathrm{~Hz}$

AQ $\quad 3.2998593 \mathrm{sec}$

DW $\quad 60.800$ usec

DE $\quad 6.50$ usec

$\begin{array}{rr}\text { TE } & 298.0 \mathrm{~K} \\ \text { D1 } & 1.00000000 \mathrm{sec}\end{array}$

TDO

$=======$ CHANNEL $\mathrm{fl}=======$

NUC1

(1.000000

P1 14.75 usec

$\begin{array}{ll}\text { PL1 } & -1.00 \mathrm{~dB}\end{array}$

SFO1 $400.1324710 \mathrm{MHz}$

F2 - Processing parameters

SI 32768

$400.1300102 \mathrm{MHz}$

$\begin{array}{lll}\mathrm{SSB} & 0 & 0.30 \mathrm{~Hz} \\ \mathrm{LB} & 0\end{array}$

10

8

8

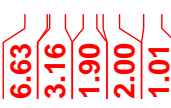

Figure S106. ${ }^{1} \mathrm{H}$ NMR (400 $\mathrm{MHz}, \mathrm{CDCl}_{3}$ ) Spectrum of $\mathbf{7 a}$ 


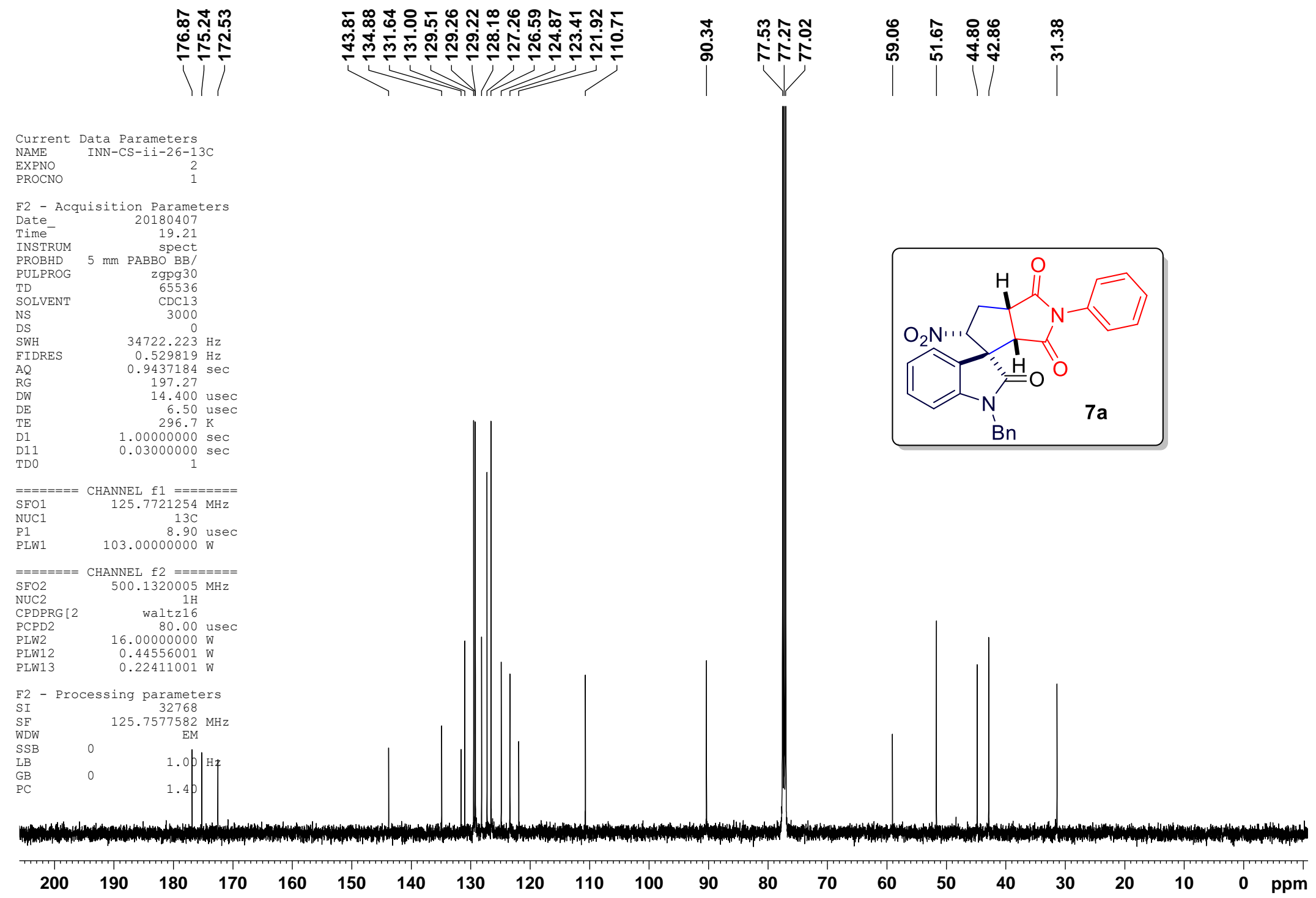

Figure S107. ${ }^{13} \mathrm{C}$ NMR $\left(125 \mathrm{MHz}, \mathrm{CDCl}_{3}\right)$ Spectrum of 7a 


||

Current
Nata Pare
EXPNO
INN-CS-II-26-APT
3

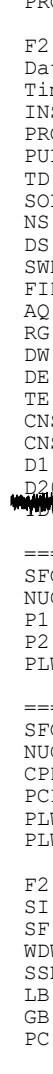

$\begin{array}{lc}\text { PROCNO } & 1 \\ \text { F2 - Acquisition Parameters } \\ \text { Date_ } & \text { 20180415 }\end{array}$

Time-
INSTRUM

spect
$\begin{aligned} & \text { INSTRUM } \\ & \text { PROBHD } \\ & \text { PULPROG }\end{aligned} 5 \mathrm{~mm}$ PABBO BB/

TD
SOLVENT
NS

$\begin{array}{lc}\text { NS } & 1756 \\ \text { DS } & 0 \\ \text { SWH } & 29761.904 \mathrm{~Hz} \\ \text { FTDRES } & 0.454131 \mathrm{~Hz}\end{array}$

$\begin{array}{lr}\text { FIDRES } & 0.454131 \mathrm{~Hz} \\ \text { AQ } & 1.1010048 \mathrm{sec} \\ \text { RG } & 197.27\end{array}$

$\begin{array}{lr}\text { RG } & 197.27 \\ \text { DW } & 16.800 \text { usec } \\ \text { DE } & 6.50 \text { usec } \\ \text { TE } & 297.8 \mathrm{~K}\end{array}$

$\begin{array}{lr}\text { TE } & 297.8 \\ \text { CNST2 } & 145.0000000 \\ \text { CNST11 } & 1.0000000\end{array}$

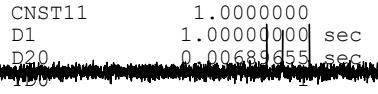

$\begin{array}{ll}======= & \text { CHANNEL } \mathrm{f} 1======= \\ \text { SFO1 } & 125.7703643 \mathrm{MHz} \\ \text { NUC1 } & 13 \mathrm{C} \\ \text { P1 } & 8.90\end{array}$

$\begin{array}{lr}\text { P1 } & 8.90 \text { use } \\ \text { P2 } & 17.80 \text { use } \\ \text { PLW1 } & 103.00000000\end{array}$

$\begin{array}{lc}======= & \text { CHANNEL } f 2======= \\ \text { SFO2 } & 500.1320005 \mathrm{MHz} \\ \text { NUC2 } & 1 \mathrm{HH} \\ \text { CDPRG } 2 & \text { waltz } 16 \\ \text { PCPD2 } & 80.00 \text { usec } \\ \text { PLW2 } & 16.00000000 \mathrm{~W} \\ \text { PIW12 } & 0.44556001\end{array}$

F2 - Processing parameters

$\begin{array}{lr}\text { SI } & 3208 \\ \text { SF } & 125.7577671 \\ \text { MHz }\end{array}$

$\begin{array}{llc}\text { WSB } & 0 & \text { EM } \\ \text { SSB } & 0.00 \mathrm{~Hz} \\ \text { IB } & 0 & 1.40\end{array}$

200190

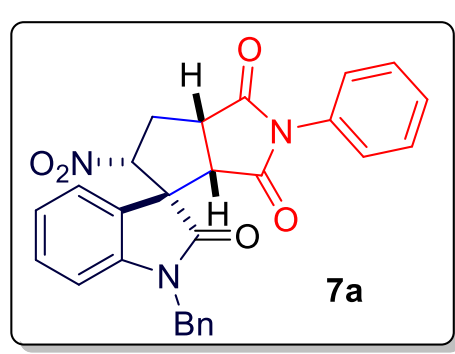

200

$190 \quad 180$

$170 \quad 160$

$150 \quad 14$

130

$120 \quad 110$

10090

$80 \quad 70$

60

$50 \quad 40$

30

20

Figure S108. ${ }^{13} \mathrm{C}$-APT NMR (125 $\left.\mathrm{MHz}^{\mathrm{C}} \mathrm{CDCl}_{3}\right)$ Spectrum of $\mathbf{7 a}$ 


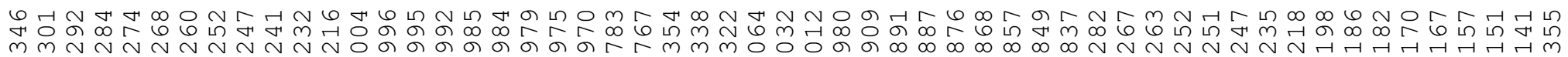

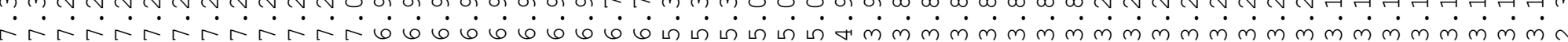

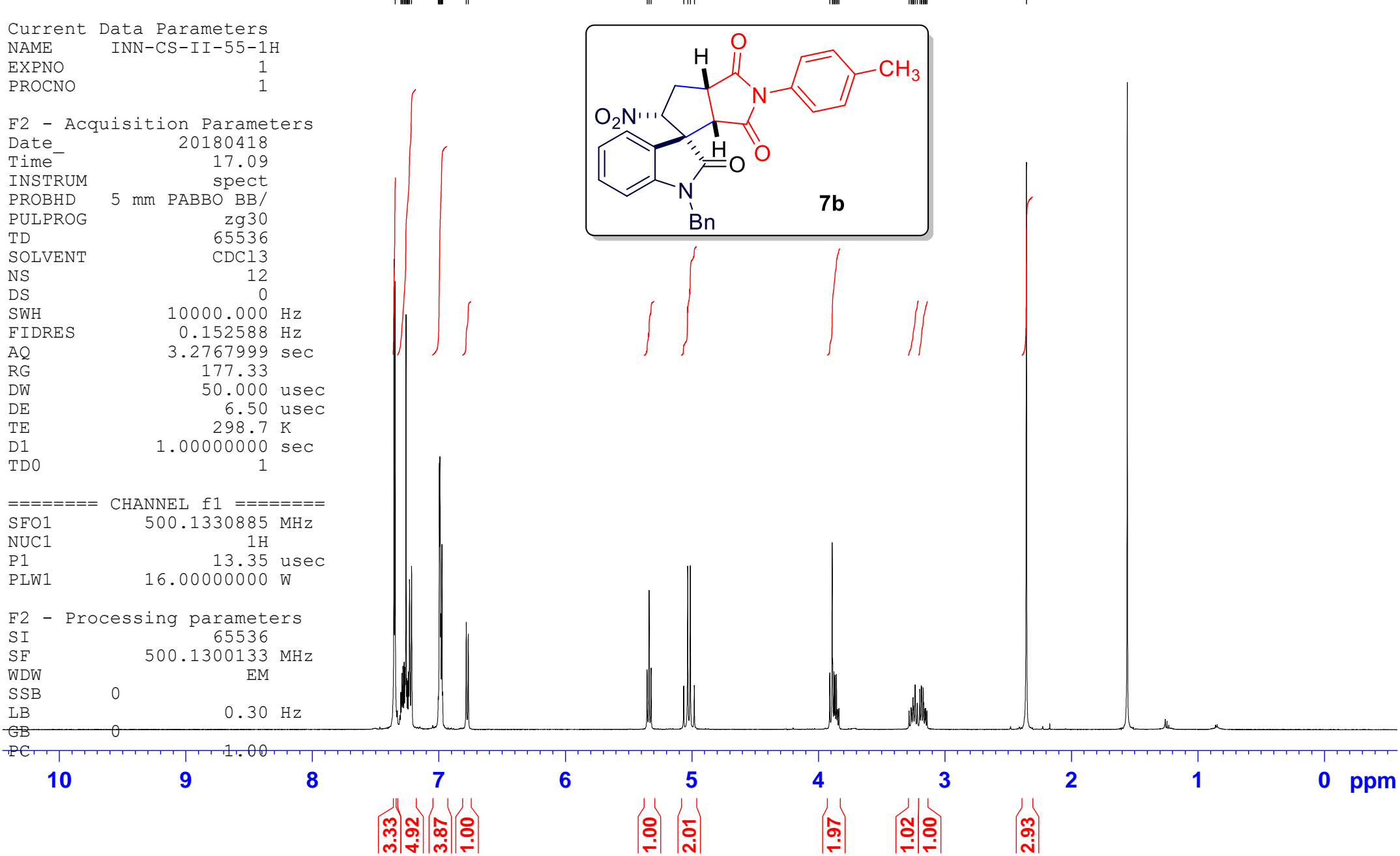

Figure S109. ${ }^{1} \mathrm{H}$ NMR (500 MHz, $\left.\mathrm{CDCl}_{3}\right)$ Spectrum of $\mathbf{7 b}$ 


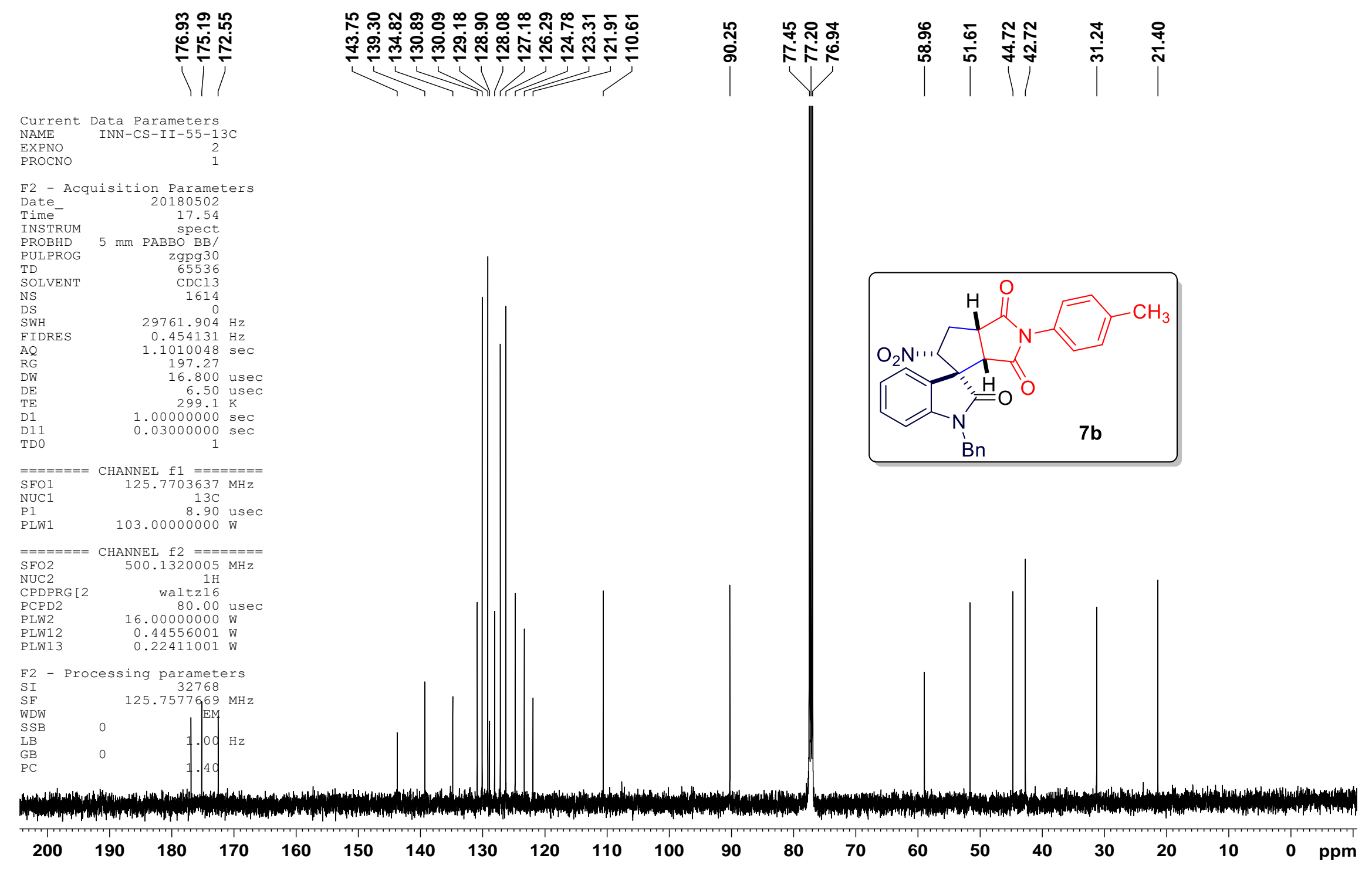

Figure S110. ${ }^{13} \mathrm{C}$ NMR (125 MHz, $\left.\mathrm{CDCl}_{3}\right)$ Spectrum of $\mathbf{7 b}$ 


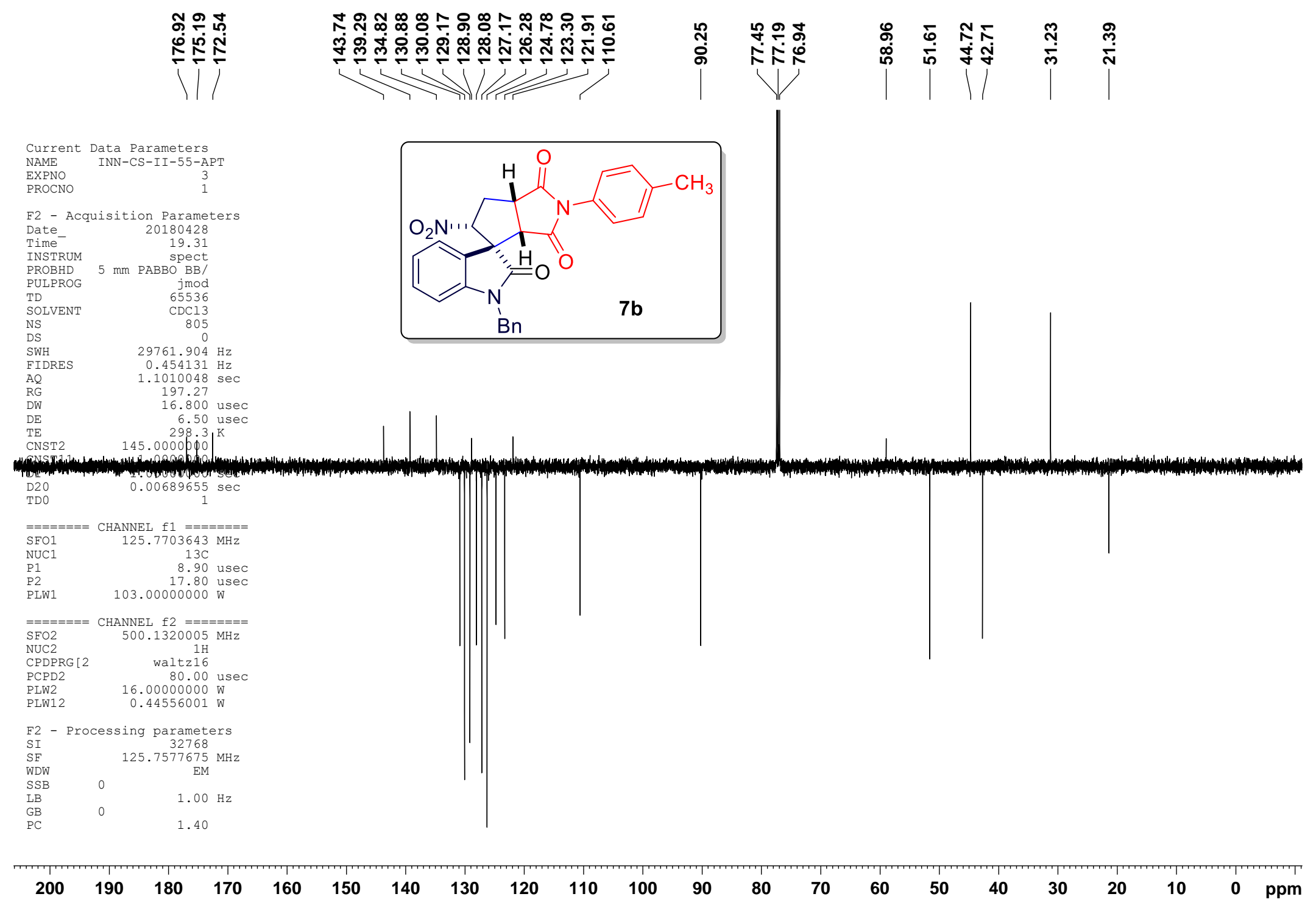

Figure S111. ${ }^{13} \mathrm{C}-\mathrm{APT}$ NMR (125 MHz, $\left.\mathrm{CDCl}_{3}\right)$ Spectrum of $\mathbf{7 b}$ 


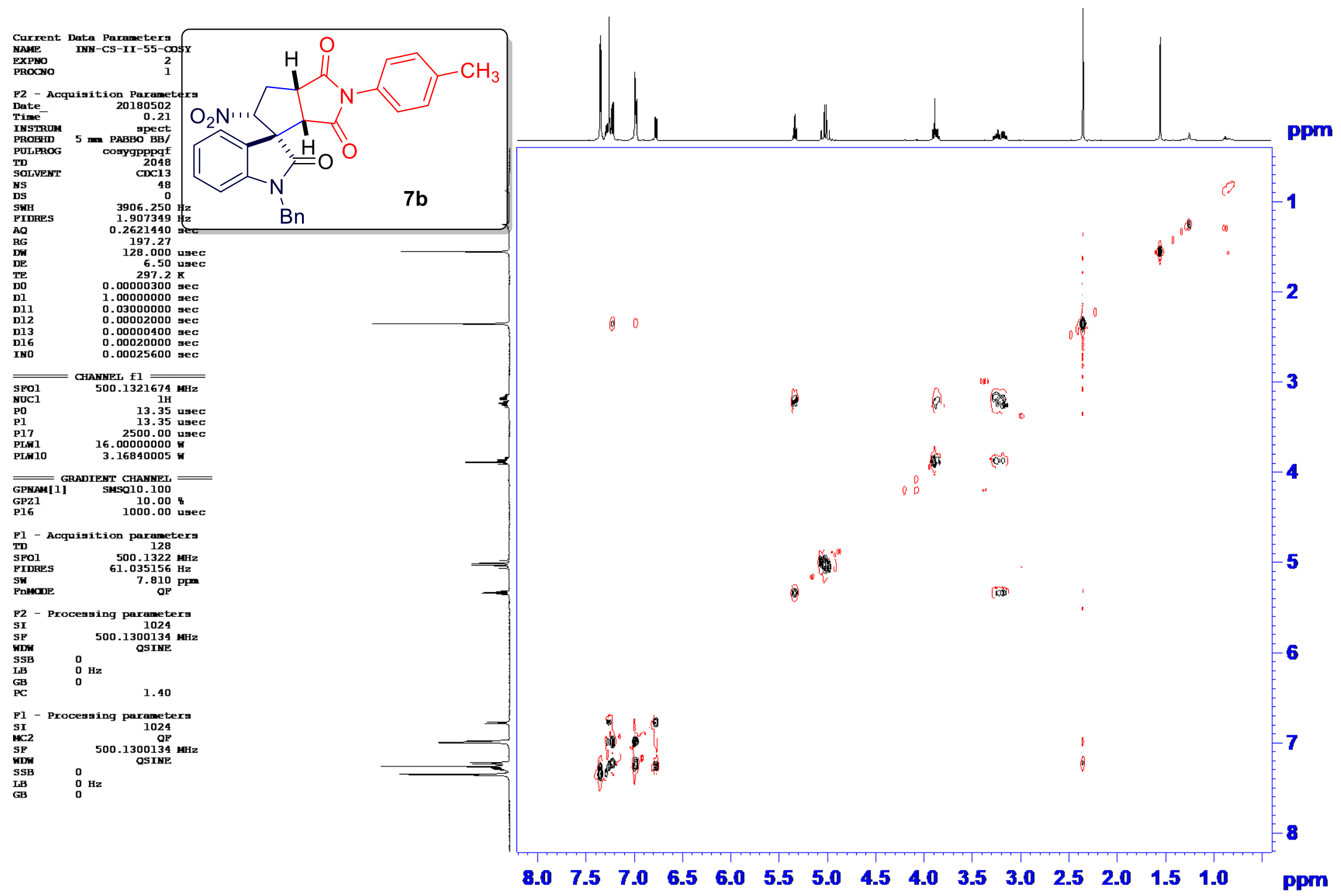

Figure S112. ${ }^{1} \mathrm{H}-{ }^{1} \mathrm{H}$ COSY NMR $\left(500 \mathrm{MHz}, \mathrm{CDCl}_{3}\right)$ Spectrum of 7b 

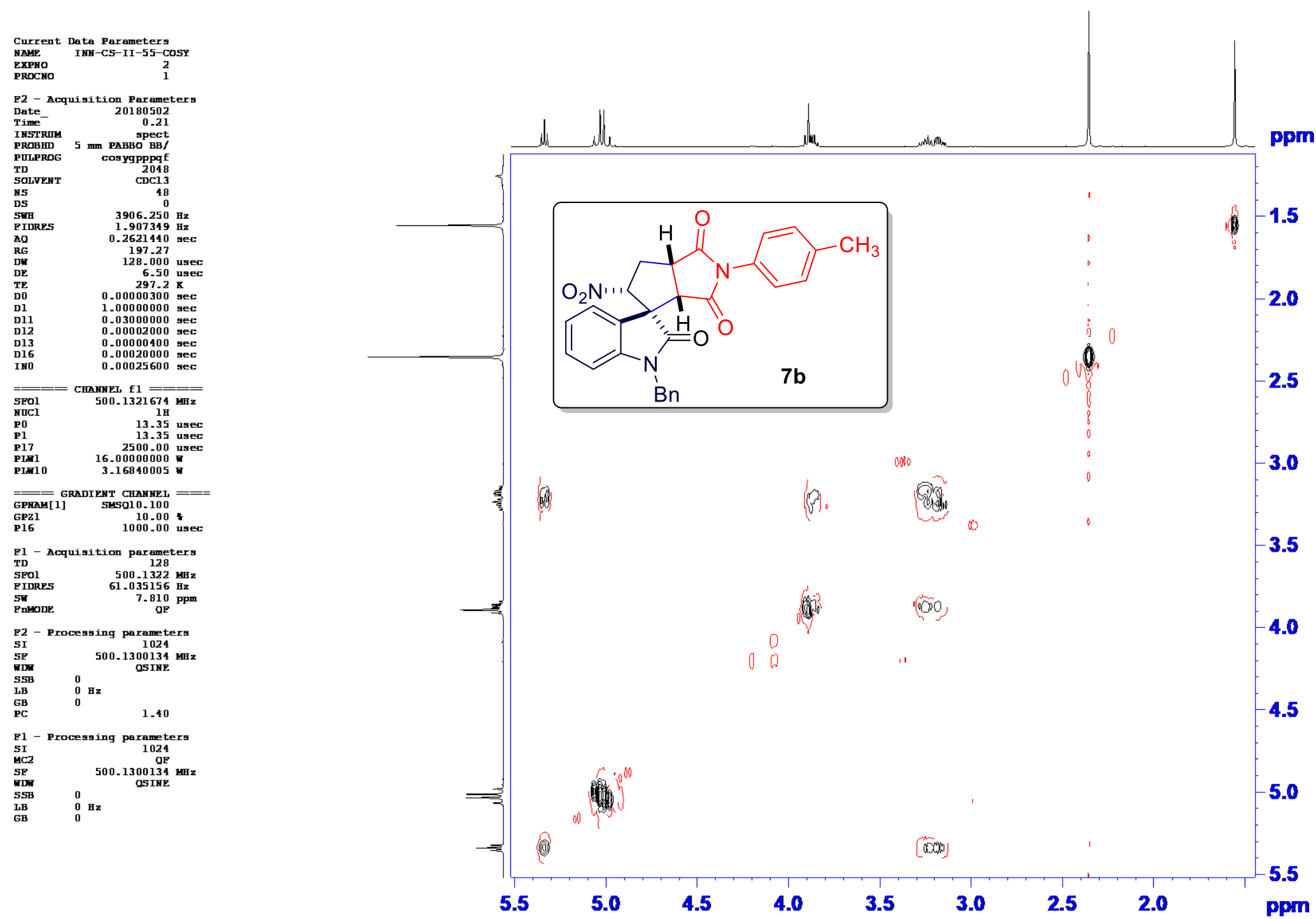

Figure S113. ${ }^{1} \mathrm{H}-{ }^{1} \mathrm{H}$ COSY NMR (500 MHz, $\left.\mathrm{CDCl}_{3}\right)$ Spectrum (Expansion) of $\mathbf{7 b}$ 


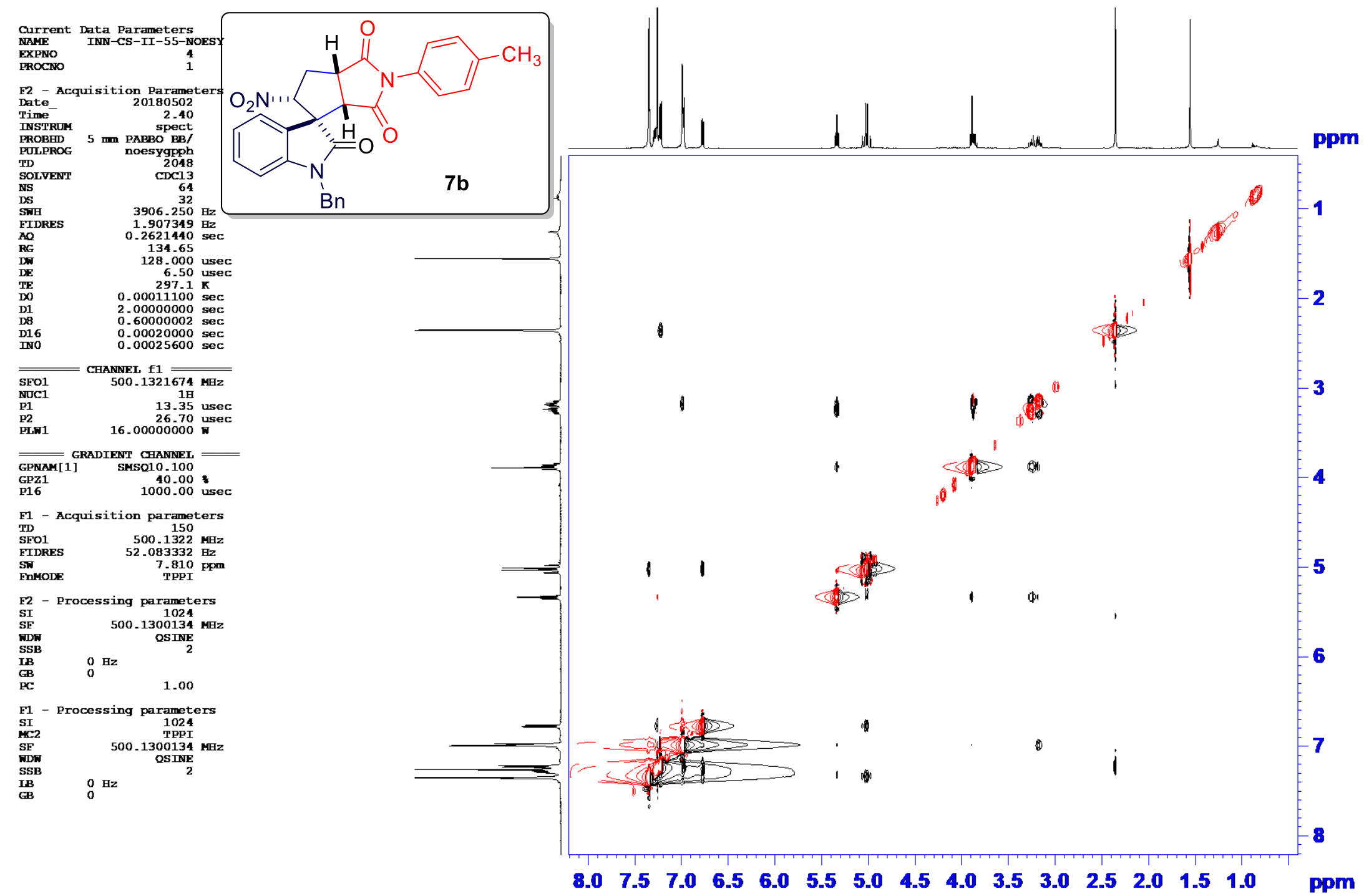

Figure S114. ${ }^{1} \mathrm{H}^{-1} \mathrm{H}$ NOESY NMR $\left(500 \mathrm{MHz}, \mathrm{CDCl}_{3}\right)$ Spectrum of $\mathbf{7 b}$ 


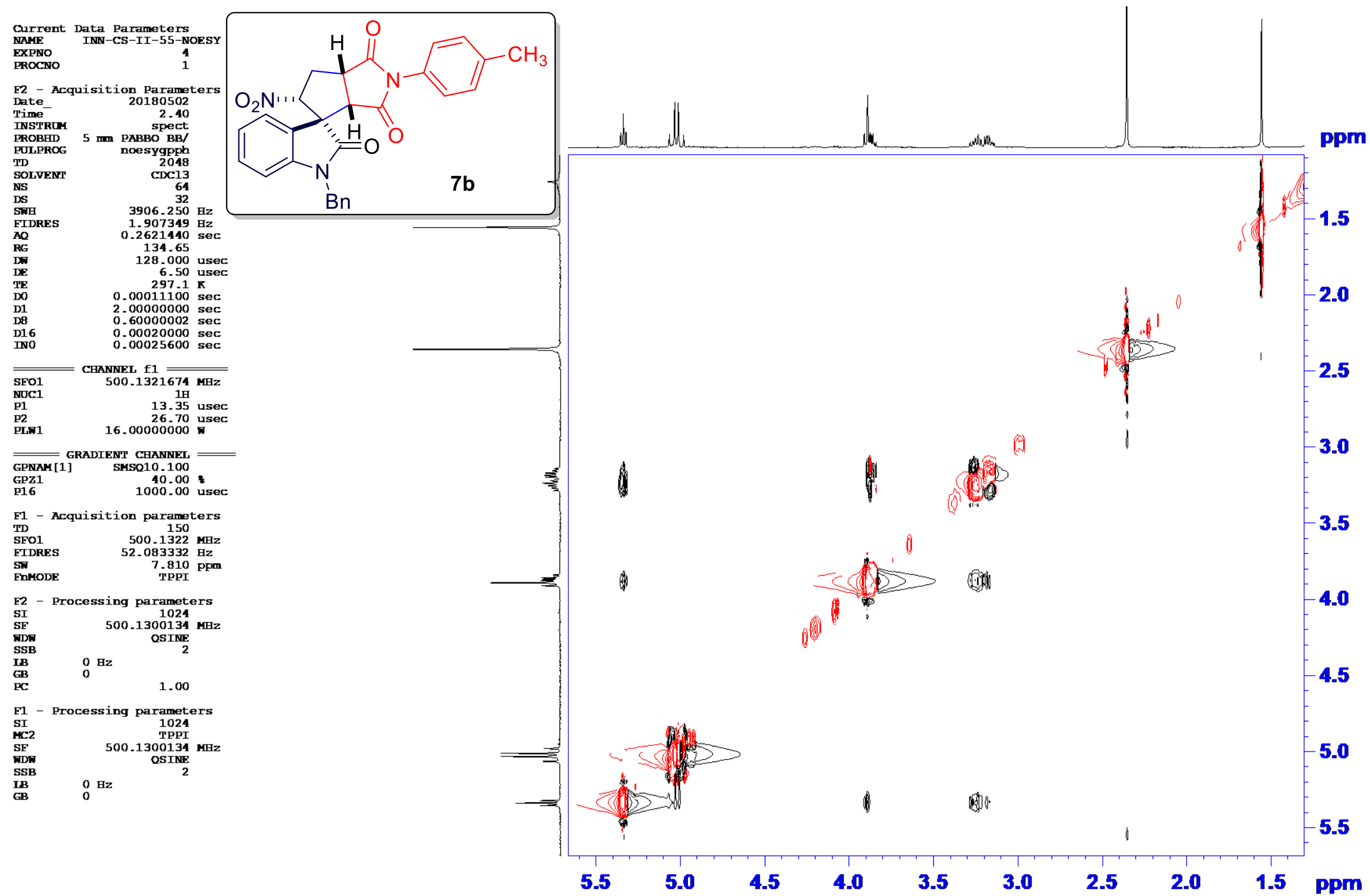

Figure S115. ${ }^{1} \mathrm{H}^{-1} \mathrm{H}$ NOESY NMR $\left(500 \mathrm{MHz}, \mathrm{CDCl}_{3}\right)$ Spectrum (Expansion) of $\mathbf{7 b}$ 


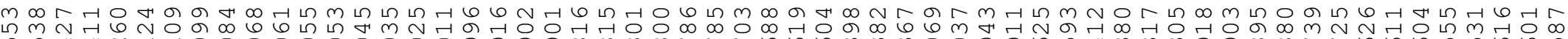

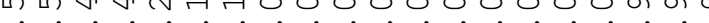

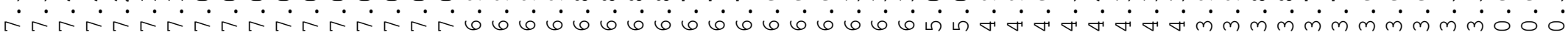

Current Data Parameters $\begin{array}{rr}\text { NAME } & \text { INN-CS-II-83-1H } \\ \text { EXPNO } & 8 \\ \text { PROCNO } & 1\end{array}$

F2 - Acquisition Parameters

Date__ 20180706

Time 20.44

PROBHD $5 \mathrm{~mm}$ PABBO BB/

$\begin{array}{lr}\text { PULPROG } & \text { zg30 } \\ \text { TD } & 65536\end{array}$

SOLVENT

NS

DS

FIDRES

$\mathrm{AQ}$

RG

DW

TE

TDO

$=======$ CHANNEL fi $========$

$$
\begin{array}{r}
65536 \\
\mathrm{CDC13} \\
10
\end{array}
$$

$10000.000 \mathrm{~Hz}$ $0.152588 \mathrm{~Hz}$ 106.54

50.000 usec 6.50 usec

$1.00000000 \mathrm{sec}$

P1 $\quad 13.35$ usec

F2 - Processing parameters

SI 65536

$500.1300137 \mathrm{MHz}$

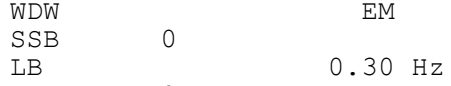

$\begin{array}{lll}\text { LB } & 0 & 0.30 \mathrm{~Hz}\end{array}$
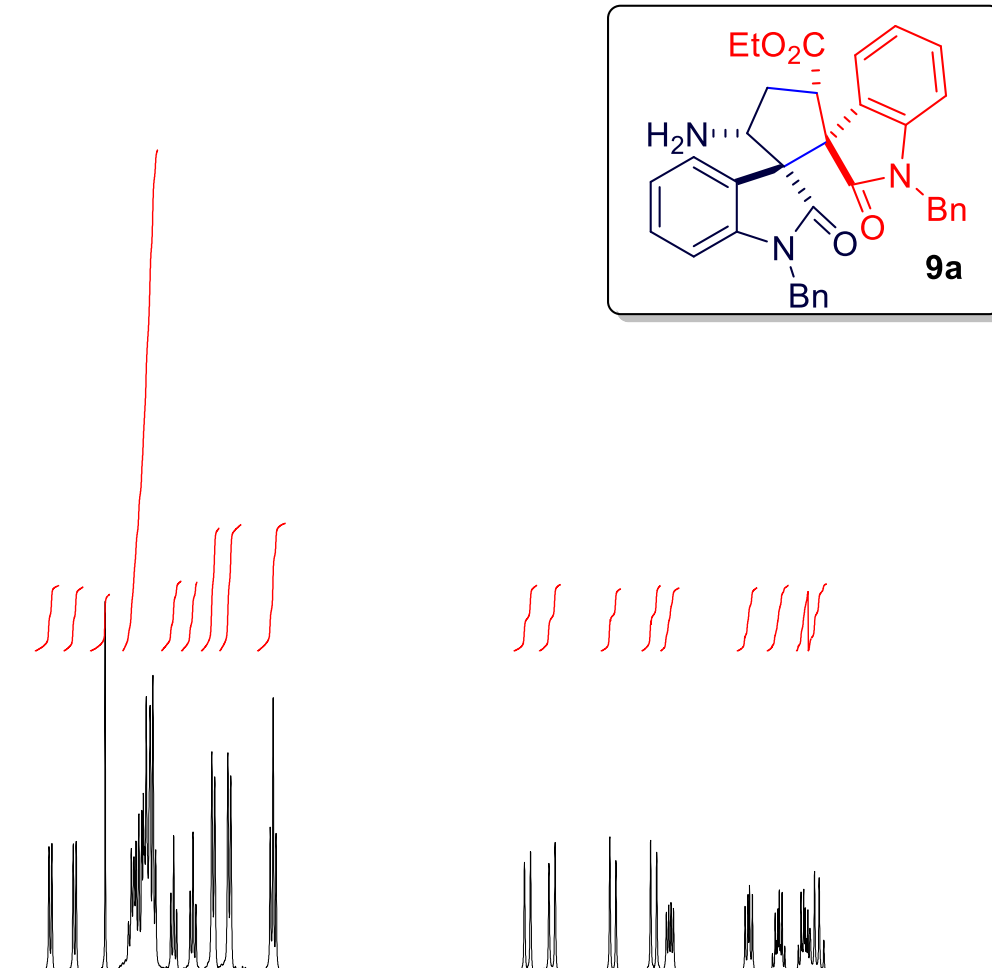

Bn

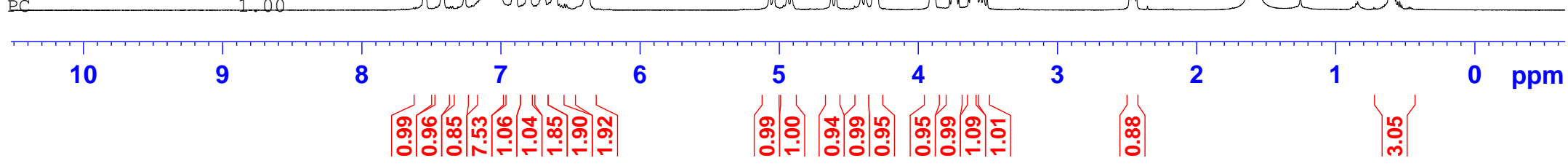

Figure S116. ${ }^{1} \mathrm{H} \mathrm{NMR}\left(500 \mathrm{MHz}, \mathrm{CDCl}_{3}\right)$ Spectrum of 9a 


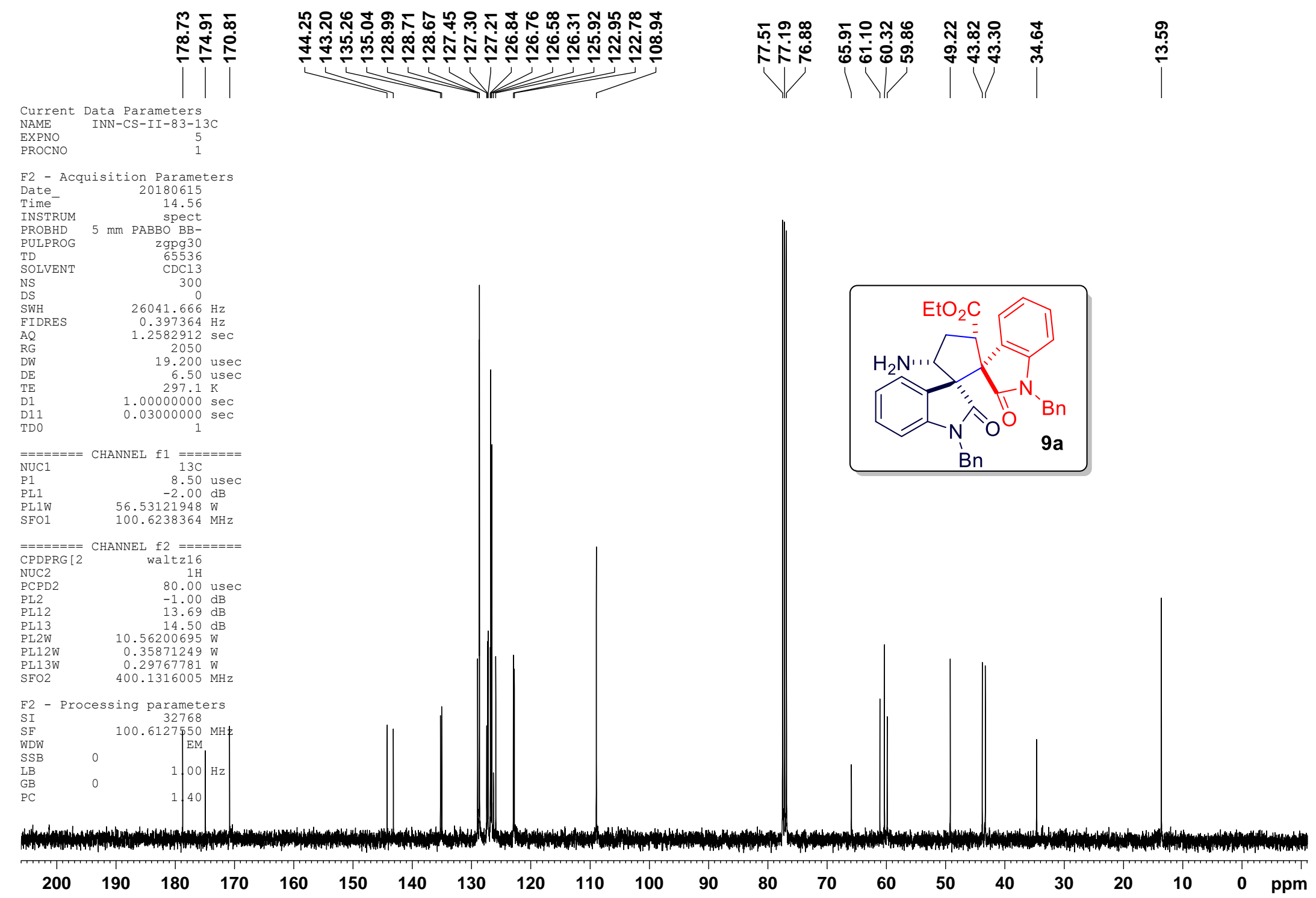

Figure S117. ${ }^{13} \mathrm{C}$ NMR (100 MHz, $\left.\mathrm{CDCl}_{3}\right)$ Spectrum of 9a 


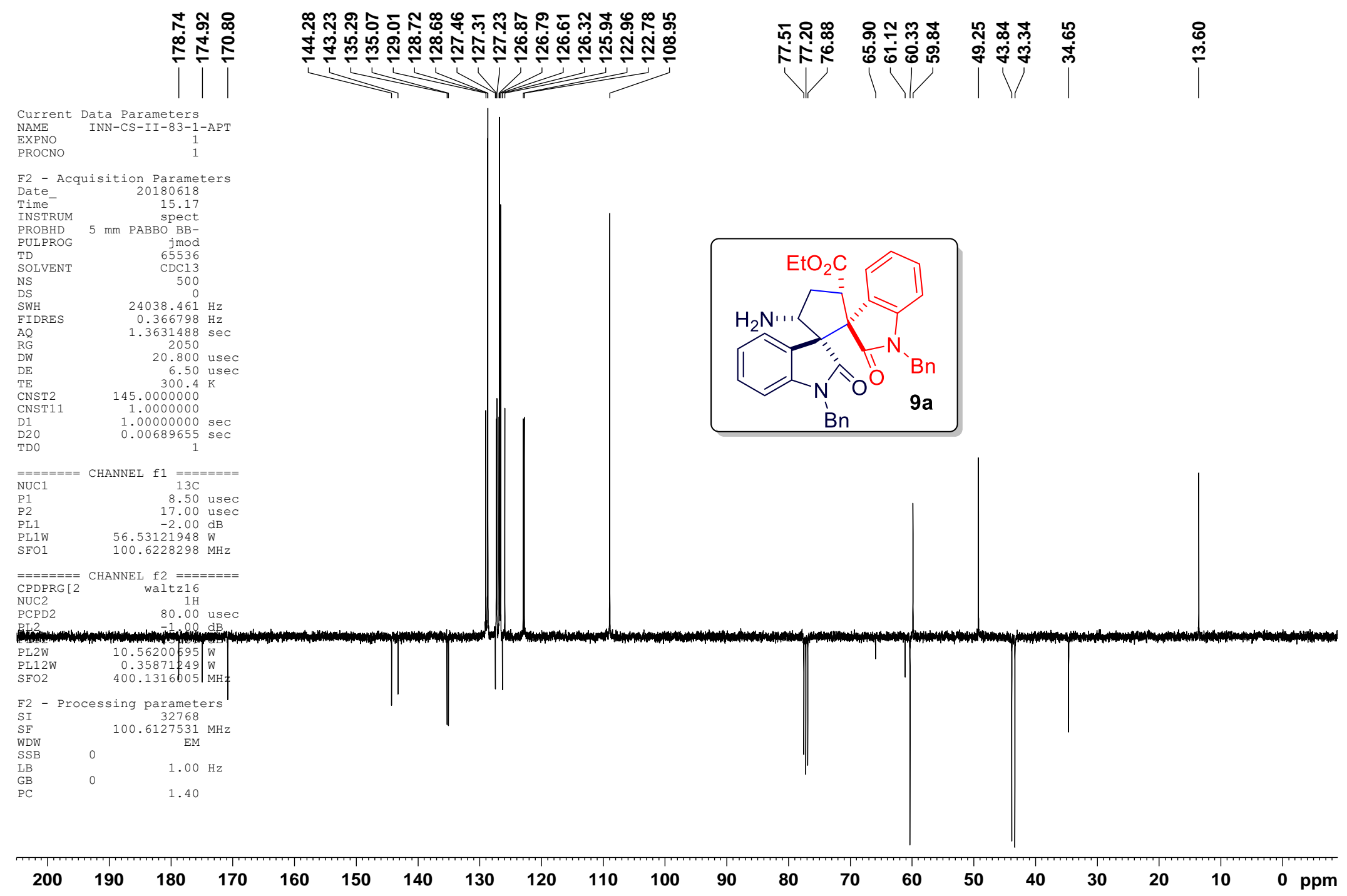

Figure S118. ${ }^{13} \mathrm{C}$-APT NMR (100 MHz, $\left.\mathrm{CDCl}_{3}\right)$ Spectrum of 9a 


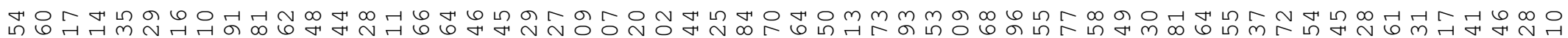
minn

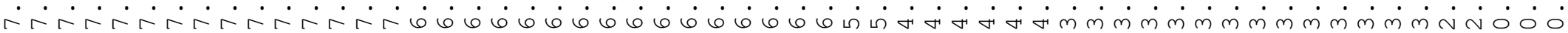

Current Data Parameters

NAME INN-CS-II-87-1H

PROCNO

F2 - Acquisition Parameters

Date_ 20180709

10.22

PROBHD $5 \mathrm{~mm}$ PABBO BB-

PULPROG

TD 54274

SOLVENT

NS

SWH

FIDRES

AQ

$\mathrm{DW}$

$\mathrm{DE}$

TE

TD0

$$
\begin{array}{r}
\mathrm{CDCl} 3 \\
23 \\
0 \\
8223.685 \mathrm{~Hz} \\
0.151522 \mathrm{~Hz} \\
3.2998593 \mathrm{sec} \\
144 \mathrm{u} \\
60.800 \mathrm{usec} \\
6.50 \mathrm{usec} \\
295.8 \mathrm{~K} \\
1.0000000 \mathrm{sec}
\end{array}
$$

$=$ CHANNEL $\mathrm{f} 1 \mathrm{=}=======$

P1 1

PL1W

$1 \mathrm{H}$

$-1.00 \mathrm{~dB}$

$10.56200695 \mathrm{~W}$

$400.1324710 \mathrm{MHz}$

F2 - Processing parameters

SI 32768

SF $\quad 400.1300100 \mathrm{MHz}$

WDW EM

$\begin{array}{lll}\mathrm{SSB} & 0 & 0.30 \mathrm{~Hz}\end{array}$

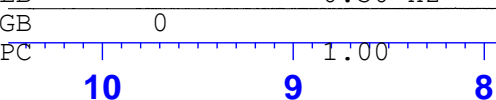

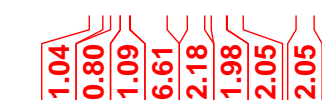

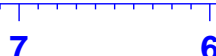

6

Figure S119. ${ }^{1} \mathrm{H}$ NMR (400 MHz, $\left.\mathrm{CDCl}_{3}\right)$ Spectrum of 9b 


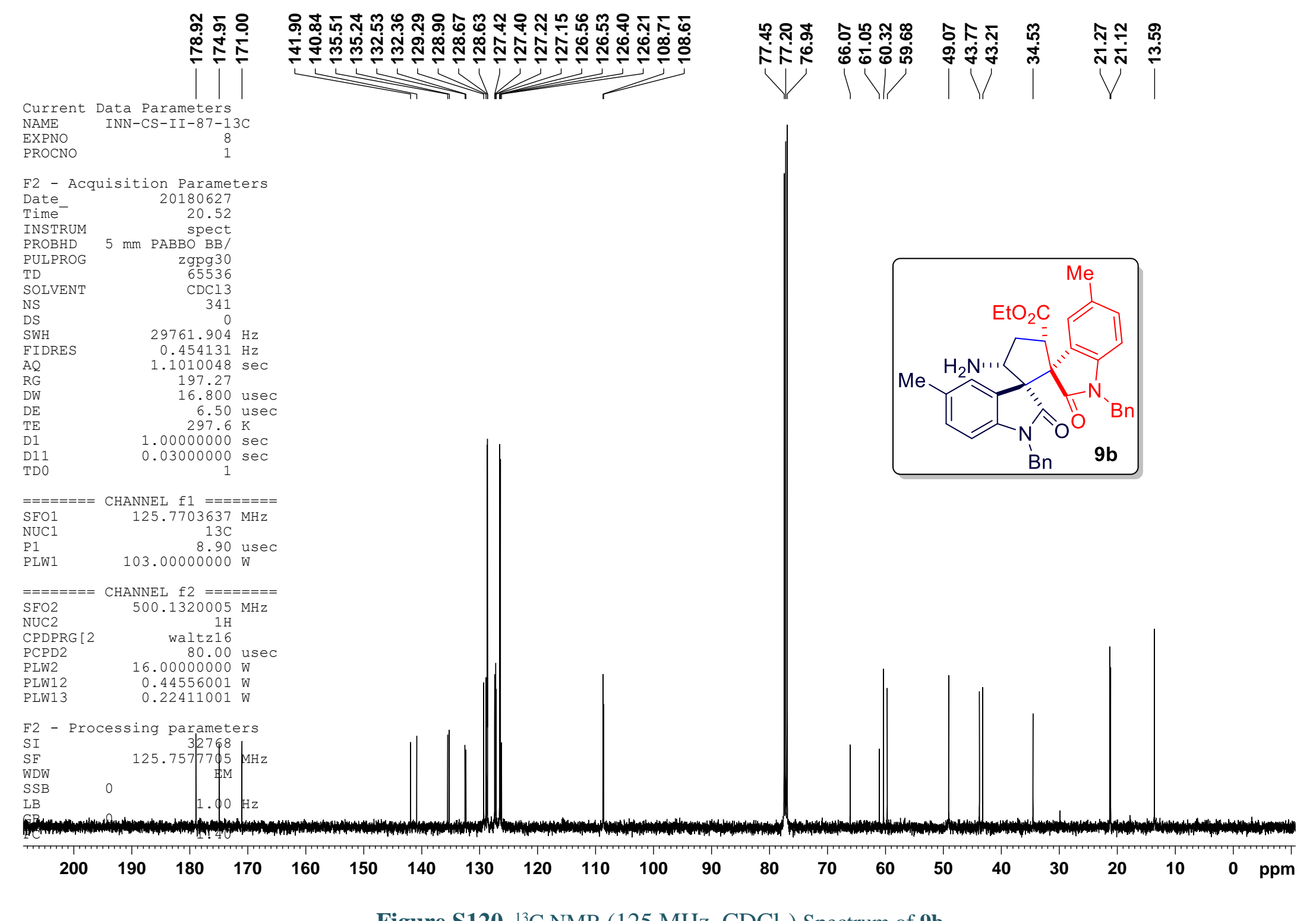

Figure S120. ${ }^{13} \mathrm{C}$ NMR $\left(125 \mathrm{MHz}, \mathrm{CDCl}_{3}\right)$ Spectrum of $9 \mathbf{b}$ 


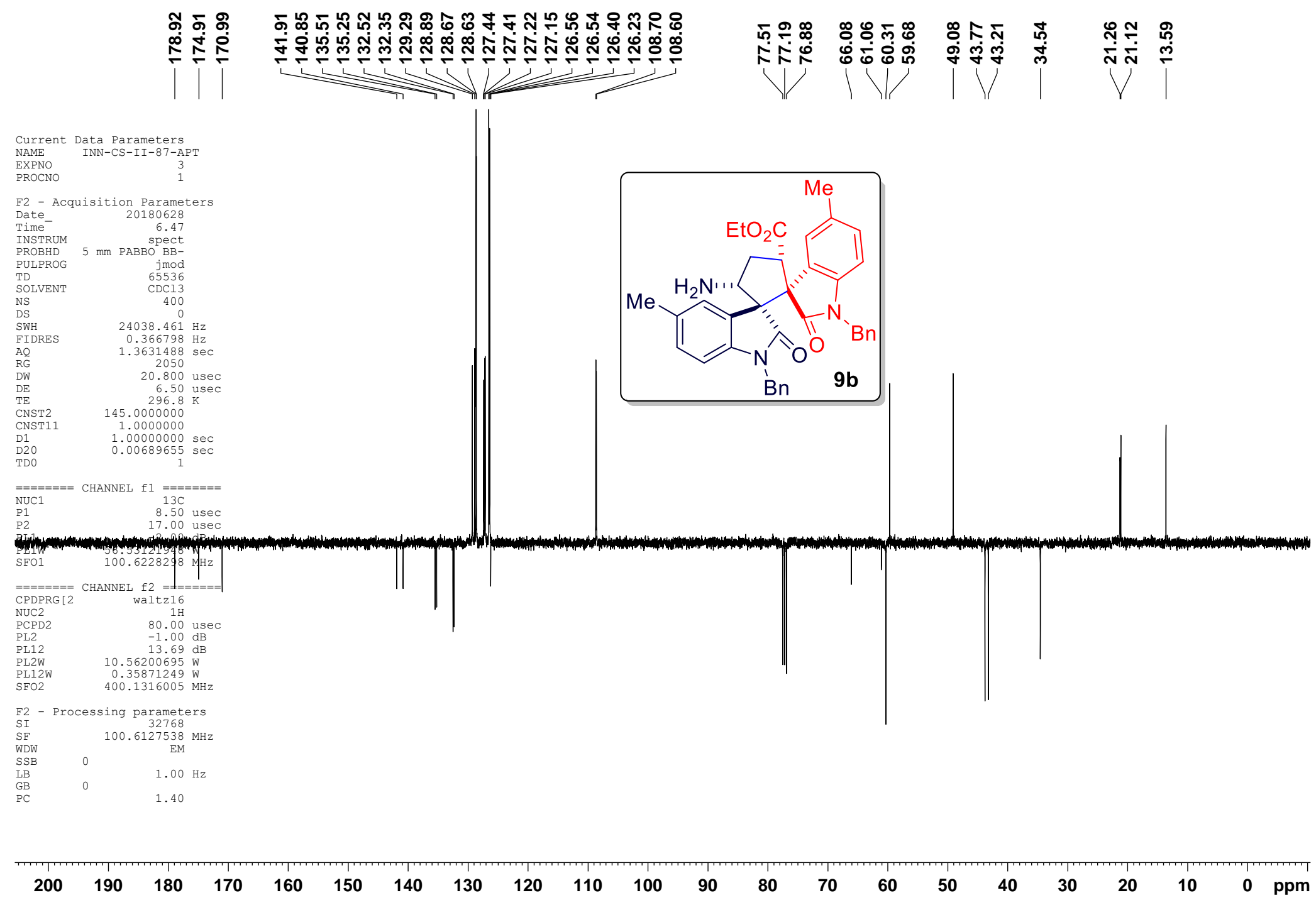

Figure S121. ${ }^{13} \mathrm{C}$-APT NMR (100 MHz, $\left.\mathrm{CDCl}_{3}\right)$ Spectrum of 9b 


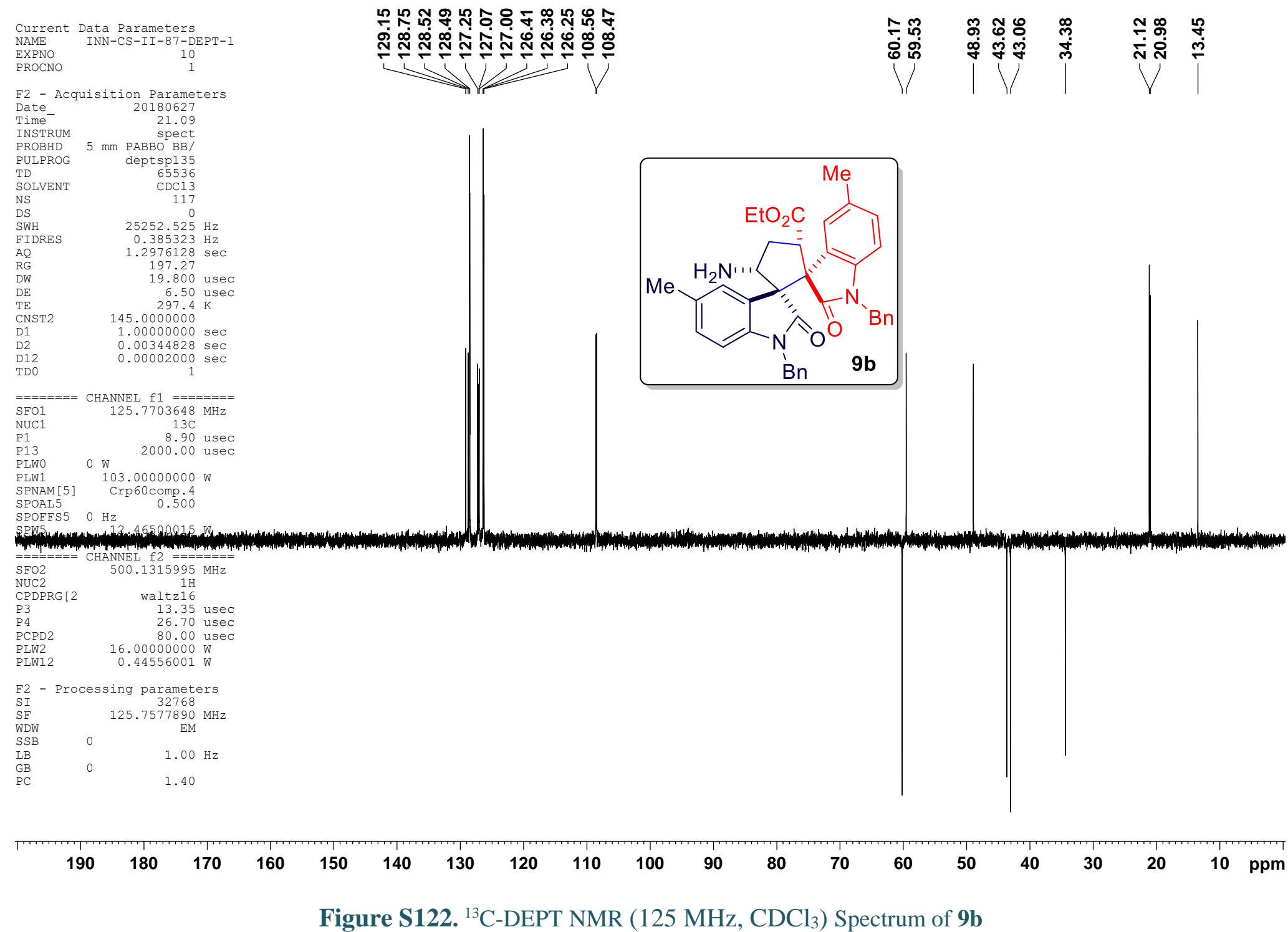

